\title{
Alguns Testes de Linearidade para Séries Temporais nos Domínios do Tempo e da Freqüência
} Alessandra Montini Ventura

\author{
DISSERTAÇÃO APRESENTADA AO \\ INSTITUTO DE MATEMÁTICA E ESTATÍSTICA \\ DA UNIVERSIDADE DE SÃO PAULO \\ PARA OBTENÇÃO DO GRAU DE MESTRE \\ EM ESTATÍSTICA
}

Área de Concentração: Estatística

Orientadora: Profa. Dra. Clélia Maria de Castro Toloi

São Paulo, março de 2000. 


\section{Alguns Testes de Linearidade para Séries Temporais nos Domínios do Tempo e da Frequiuencia}

Este exemplar corresponde à redação final da dissertação devidamente corrigida e defendida por Alessandra Montini Ventura e aprovada pela comissão julgadora.

São Paulo, 31 de março de 2000.

Banca examinadora:

Professora Dra. Clélia Maria de Castro Toloi (Orientadora) - IME - USP

Professor Dr. Pedro Alberto Morettin - IME - USP

Professor Dr. Afrânio R. Mesquita - IO - USP 
Para minhas sobrinhas Elisa e Sabrina que são as fontes de alegria da minha vida. 


\section{Agradecimentos}

A Deus por ter me dado força e coragem.

Aos meus pais, pelo carinho e amor despendidos por mim nestes anos, meu eterno obrigada.

Ao meu marido Gilberto pela paciência, companheirismo e amor, obrigada.

Às minhas tias Mirta e Miriam e a minha avó D.Benta pela ajuda e apoio nos momentos dificeis.

Sou grata em especial ao Prof. Canton pela amizade, confiança, incentivo e por sempre acreditar em mim, muito obrigada.

Agradeço também minhas grandes amigas Carine e Maria Paula pelos bons momentos em que vivemos e pelos momentos dificeis que passamos juntas nesta grande caminhada.

Agradeço também à minha orientadora Clélia pela amizade e orientação. 


\section{Resumo}

Um problema prático, encontrado ao ajustar um modelo a uma série temporal, é decidir se devemos utilizar, ou não, um modelo linear. Os testes de linearidade podem ser utilizados para decidir quando devemos usar a classe dos modelos lineares cuja identificação, estimação e previsão está amplamente desenvolvida e implementada nos programas computacionais. Este trabalho tem como objetivo descrever e aplicar a algumas série reais os testes de linearidade e normalidade de Subba Rao e Gabr (1980), Hinich (1982), McLeod e Li (1983), Tsay (1986), Poggi e Portier (1997) e Brillinger e Irizarry (1998).

\section{Abstract}

A practical problem, found when fitting a model to a time series, is to decide if we must use, or not, a linear model. The linearity tests can be used to decide when we must use the class of the linear models whose identification, estimation and forecast are widely developed and implemented in the computational programs. This work has as objective to describe and to apply to some series the tests of linearity and normality of Subba Rao and Gabr (1980), Hinich (1982), McLeod and Li (1983), Tsay (1986), Poggi and Portier (1997) and Brillinger and Irizarry (1998). 


\section{Conteúdo}

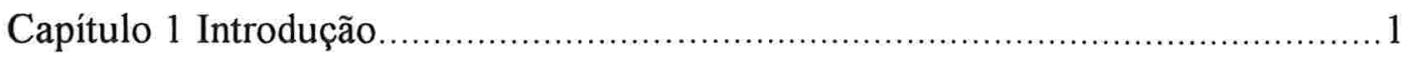

Capítulo 2 Definições e alguns resultados ...........................................................

Capítulo 3 Modelos lineares em séries temporais …………………………........13

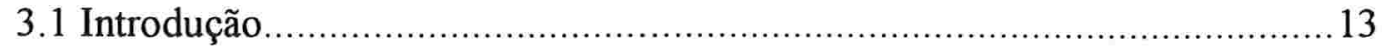

3.2 Modelo auto-regressivo de médias móveis …………...............................

3.3 Extensões do modelo auto-regressivo de médias móveis .............................. 16

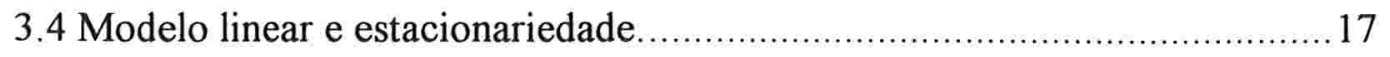

Capítulo 4 Alguns modelos não-lineares em séries temporais ................................19

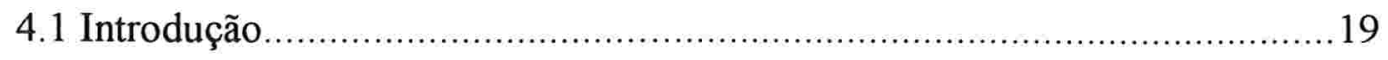

4.2 Algumas características importantes de sistemas não-lineares .....................20

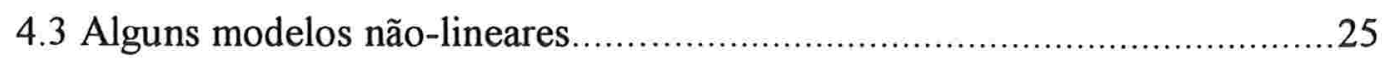

Capítulo 5 Testes de linearidade e normalidade no domínio de freqüências .........43

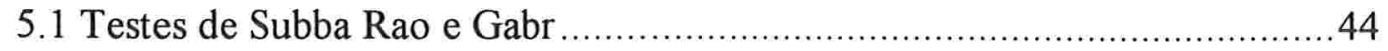

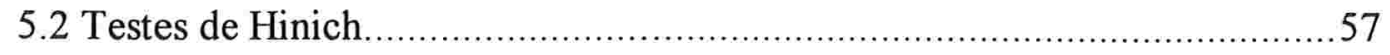

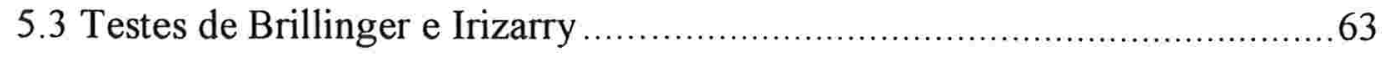


Capítulo 6 Testes de linearidade no domínio do tempo 68

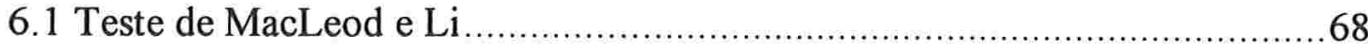

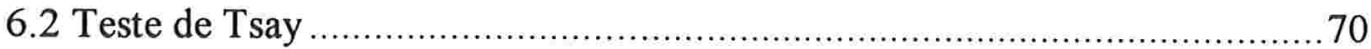

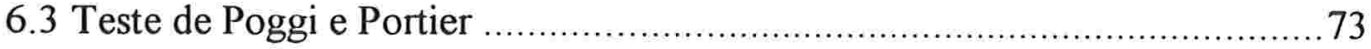

6.4 Outros testes de linearidade ................................................................. 78

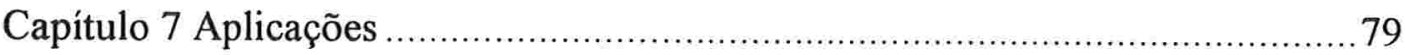

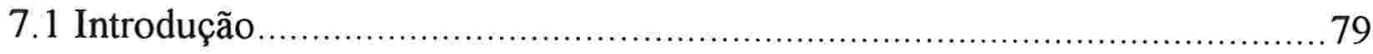

7.2 Total de óbitos por causas respiratórias ............................................... 82

7.3 Total de óbitos para crianças de 1 a 11 meses ...........................................91

7.4 Média da concentração do agente poluidor PM10 ................................ 100

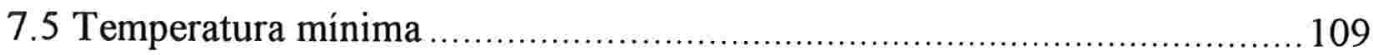

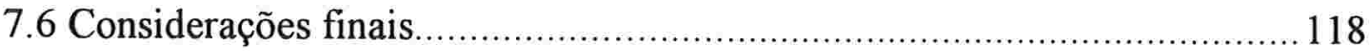

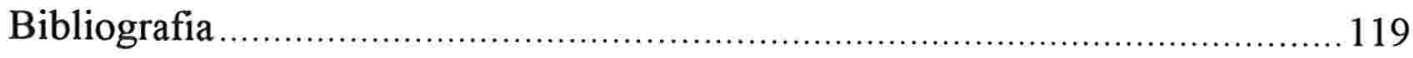

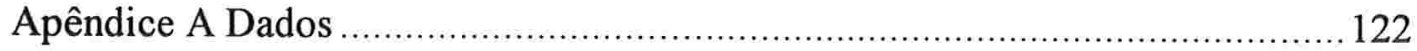

Apêndice B Programas ............................................................................. 131 


\section{Capítulo 1}

\section{Introdução}

Muitos fenômenos físicos e o comportamentos de muitas séries financeiras não podem ser representados adequadamente pelos modelos lineares e por esta razão, surge a necessidade do estudo dos modelos não-lineares e dos espectros de altas ordens que fornecem informações importantes sobre as séries não-lineares. Quando a série é linear o espectro de segunda ordem contém toda a informação presente na série e, quando a série é não-linear, o espectro de segunda ordem não capta as características de não linearidade e, desta forma, surge a necessidade do estudo dos espectros de altas ordens para detectar as características de linearidade e não normalidade. O tipo mais simples de análise espectral de alta ordem é a análise bi-espectral, cuja literatura está amplamente desenvolvida e é a base para os testes de linearidade e normalidade no domínio da freqüências que serão desenvolvidos neste trabalho.

Neste trabalho abordamos os testes de linearidade baseados no domínio do tempo de McLeod e Li (1983), Tsay (1986) e Poggi e Portier (1997) e os testes de linearidade e normalidade baseados no domínio de freqüências de Subba Rao e Gabr (1980), Hinich (1982) e Brillinger e Irizarry (1998).

No Capítulo 2 apresentamos algumas definições e resultados que serão utilizados e citados nos capítulos posteriores. 
No Capítulo 3 abordamos os modelos lineares, enquanto que no Capítulo 4 abordamos alguns modelos não-lineares e algumas das características importantes destes modelos.

No Capítulo 5 descrevemos alguns testes de linearidade e normalidade no domínio de freqüências e no Capítulo 6 apresentamos alguns testes de linearidade no domínio do tempo.

Por fim, no Capítulo 7 fazemos uma aplicação dos testes citados anteriormente às séries de concentração de poeira, temperatura e óbitos da cidade de São Paulo. 


\section{Capítulo 2}

\section{Definições e alguns resultados}

Definição 2.1: Um processo estocástico $X=\left\{X_{t, \theta} ; t \in T, \theta \in \Omega\right\}$ é um conjunto de variáveis aleatórias definidas sobre um espaço de probabilidade $(\Omega, \Im, P)$ e indexadas pelos elementos do conjunto de parâmetros T. O conjunto $\mathrm{T}$ é usualmente o conjunto dos números inteiros ou o conjunto dos números reais. Para cada $\theta \in \Omega$ fixado temos uma série temporal $\mathrm{X}_{\mathrm{t}, \theta}$. Visando a simplificação da notação, a partir desse momento, a série temporal $X_{t, \theta}$ será denotada por $X_{t}$.

Definição 2.2: As distribuições finito-dimensionais de uma série temporal $X_{t}$ são dadas por

$$
F\left(x_{1}, \ldots, x_{n} ; t_{1}, \ldots, t_{n}\right)=P\left(X_{t_{1}} \leq x_{1}, \ldots, X_{t_{n}} \leq x_{n}\right)
$$

em que $n \geq 1 ; t_{1}, \ldots, t_{n} \in T$ e $x_{1}, \ldots, x_{n} \in \mathbb{R}$.

Definição 2.3: As funções média, variância, autocovariância e autocorrelação de uma série temporal são dadas por 


$$
\begin{gathered}
E\left(X_{t}\right)=\int_{-\infty}^{+\infty} X d F(x ; t)=\mu_{t} ; \\
\operatorname{Var}\left(X_{t}\right)=\int_{-\infty}^{+\infty}\left(X-\mu_{t}\right)^{2} d F(x ; t)=\gamma_{x}(t, t) ; \\
\operatorname{Cov}\left(X_{j}, X_{k}\right)=\int_{-\infty}^{+\infty} \int_{-\infty}^{+\infty}\left(X_{j}-\mu_{j}\right)\left(X_{k}-\mu_{k}\right) d F\left(x_{j}, x_{k} ; j, k\right)=\gamma_{x}(j, k) ; \\
\rho_{x}(j, k)=\frac{\gamma_{x}(j, k)}{\sqrt{\gamma_{x}(j, j)} \sqrt{\gamma_{x}(k, k)}} .
\end{gathered}
$$

Definição 2.4: Uma série temporal $\mathrm{X}_{\mathrm{t}}, \mathrm{t} \in \mathrm{T}$, é estritamente estacionária se todas as distribuições finito-dimensionais forem invariantes sob translações em $t$, ou seja,

$$
F\left(x_{1}, \ldots, x_{n} ; t_{1}, \ldots, t_{n}\right)=F\left(x_{1}, \ldots, x_{n} ; t_{1}+\Delta t, \ldots, t_{n}+\Delta t\right)
$$

para quaisquer $\mathrm{t}_{1}, \ldots, \mathrm{t}_{\mathrm{n}}, \Delta \mathrm{t} \in \mathrm{T}$.

Definição 2.5: Uma série temporal $\mathrm{X}_{\mathrm{t}}, \mathrm{t} \in \mathrm{T}$, é fracamente estacionária se $\mathrm{e}$ somente se

$$
\begin{gathered}
E\left(X_{t}\right)=\mu_{t}=\mu, \forall t \in T ; \\
\operatorname{Var}\left(X_{t}\right)=\sigma^{2}<\infty, \forall t \in T ; \\
\gamma_{x}(j, k)=\gamma(|j-k|) .
\end{gathered}
$$

Isto quer dizer que $\gamma_{\mathrm{x}}(\mathrm{j}, \mathrm{k})$ depende apenas da defasagem entre $\mathrm{j}$ e $\mathrm{k}$ e, consequentemente, $\rho_{x}(j, k)=\rho(j-k \mid)$. 
Definição 2.6: Um processo estocástico $X_{t}$ diz-se gaussiano, ou normal, se para qualquer conjunto $t_{1}, \ldots, t_{n} \in T$, as variáveis aleatórias $X_{t_{1}}, \ldots, X_{t_{n}}$ possuem distribuição conjunta normal n-variada, isto é,

$$
f(\mathbf{X})=\frac{1}{(2 \pi)^{n / 2}} \mid C^{-1 / 2} \exp \left(-(\mathbf{X}-\mu)^{\prime} \mathbf{C}^{-1}(\mathbf{X}-\mu) / 2\right)
$$

em que $X=\left(X_{t_{1}}, \ldots, X_{t_{n}}\right)^{\prime}, C=\{C(s, t), s, t=0,1, \ldots, n-1\}$ é a matriz de covariâncias de $X$ e $\mu$ é o valor esperado do vetor $\mathbf{X}$.

Definição 2.7: Um processo de variáveis aleatórias independentes $e_{t}, t \in T$, é chamado de ruido branco se e somente se

$$
E\left(e_{t}\right)=\mu \text { e } \operatorname{Var}\left(e_{t}\right)=\sigma_{e}^{2} .
$$

Definição 2.8: Seja $\left\{X_{t}, t=0, \pm 1, \pm 2, \ldots\right\}$ uma série temporal estacionária até ordem k, satisfazendo $\sum_{s_{1}=-\infty}^{+\infty} \ldots \sum_{s_{k-1}=-\infty}^{+\infty}\left|C\left(s_{1}, \ldots, s_{k-1}\right)\right|<\infty$, em que $C\left(s_{1}, \ldots, s_{k-1}\right)=E\left(X_{t}-\mu\right)\left(X_{t+s 1}-\right.$ $\mu) \ldots\left(X_{t+s k-1}-\mu\right)$. O poliespectro de ordem $k$, nas freqüências $\omega_{1}, \omega_{2}, \ldots, \omega_{\mathrm{k}-1}$ é dado por

$$
f_{k}\left(\omega_{1}, \omega_{2}, \ldots, \omega_{k-1}\right)=\left(\frac{1}{2 \pi}\right)^{k-1} \sum_{s_{1}=-\infty}^{+\infty} \ldots \sum_{s_{k-1}=-\infty}^{+\infty} \mathrm{C}\left(s_{1}, \ldots, s_{k-1}\right) \exp \left(-i\left(\omega_{1} s_{1}+\ldots+\omega_{k-1} s_{k-1}\right)\right),
$$

em que $-\pi \leq \omega_{\mathrm{j}} \leq \pi$ e $\mathrm{j}=1, \ldots, \mathrm{k}-1$. A quantidade $\mathrm{C}\left(\mathrm{s}_{1}, \ldots, \mathrm{s}_{\mathrm{k}-1}\right)$ é denominada autocovariância de k-ésima ordem.

\section{Casos particulares:}

i) No caso em que $\mathrm{k}=2$, temos a função densidade espectral 


$$
\mathrm{f}_{\mathrm{x}}(\omega)=\frac{1}{2 \pi} \sum_{\mathrm{s}=-\infty}^{+\infty} \gamma(\mathrm{s}) \exp (-\mathrm{i} \omega \mathrm{s})
$$

em que $\gamma(s)=\operatorname{Cov}\left(X_{t}, X_{t+s}\right)$ e $-\pi \leq \omega \leq \pi$.

Se $X_{t}$ for um processo linear e gaussiano então $f_{x}(\omega)$ irá conter toda a informação do processo.

ii) No caso em que $\mathrm{k}=3$, temos a função densidade bi-espectral

$$
\mathrm{f}_{\mathrm{x}}\left(\omega_{1}, \omega_{2}\right)=\left(\frac{1}{(2 \pi)^{2}}\right) \sum_{\mathrm{s}_{1}=-\infty}^{+\infty} \sum_{\mathrm{s}_{2}=-\infty}^{+\infty} \mathrm{C}\left(\mathrm{s}_{1}, \mathrm{~s}_{2}\right) \exp \left(-\mathrm{i}\left(\mathrm{s}_{1} \omega_{1}+\mathrm{s}_{2} \omega_{2}\right)\right)
$$

para $-\pi \leq \omega_{1}, \omega_{2} \leq \pi$

As funções densidade bi-espectral e autocovariância de terceira ordem satisfazem a propriedade de simetria, isto é,

$$
\mathrm{f}_{\mathrm{x}}\left(\omega_{1}, \omega_{2}\right)=\mathrm{f}_{\mathrm{x}}\left(\omega_{2}, \omega_{1}\right)=\mathrm{f}_{\mathrm{x}}\left(\omega_{1},-\omega_{1}-\omega_{2}\right)=\mathrm{f}_{\mathrm{x}}\left(-\omega_{1},-\omega_{2}\right)^{*}=\mathrm{f}_{\mathrm{x}}\left(-\omega_{1}-\omega_{2}, \omega_{2}\right)
$$

$\mathrm{e}$

$$
\mathrm{C}\left(\mathrm{s}_{1}, \mathrm{~s}_{2}\right)=\mathrm{C}\left(\mathrm{s}_{2}, \mathrm{~s}_{1}\right)=\mathrm{C}\left(-\mathrm{s}_{1}, \mathrm{~s}_{2}-\mathrm{s}_{1}\right)=\mathrm{C}\left(\mathrm{s}_{1}-\mathrm{s}_{2}, \mathrm{~s}_{2}\right)
$$

em que * denota o conjugado .

A propriedade mais importante do poliespectro é que todo poliespectro de ordem maior ou igual a dois é igual a zero quando $\mathrm{X}_{\mathrm{t}}$ é um processo gaussiano.

Definição 2.9: A transformada discreta de Fourier, de uma série temporal estacionária $\left\{X_{t}, t=0,1, \ldots, T-1\right\}$ é dada por 


$$
d^{T}\left(\omega_{k}\right)=\sum_{t=0}^{T-1} X_{1} \exp \left(-i \omega_{K} t\right) \quad k=0,1, \ldots, T-1
$$

em que $\omega_{\mathrm{k}}=\frac{2 \pi \mathrm{k}}{\mathrm{T}}$

\section{Resultado}

A transformada discreta de Fourier possui a seguinte distribuição assintótica

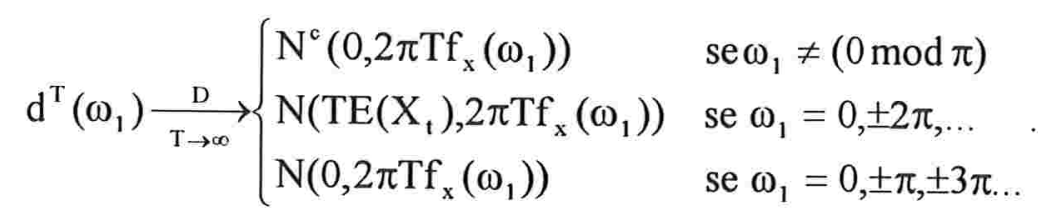

Definição 2.10: Seja $\left\{\mathrm{X}_{\mathrm{t}}, \mathrm{t}=0,1, \ldots, \mathrm{T}-1\right\}$ uma amostra de tamanho $\mathrm{T}$ de série temporal estacionária. O periodograma, estimador natural para a função densidade espectral, $\mathrm{f}_{\mathrm{x}}(\omega)$, é dado por

$$
I^{T}\left(\omega_{k}\right)=\frac{\left|d^{T}\left(\omega_{k}\right)\right|^{2}}{2 \pi T} \quad k=0, \ldots, T-1,
$$

em que $\omega_{\mathrm{k}}=\frac{2 \pi \mathrm{k}}{\mathrm{T}}$

\section{Resultado}

O periodograma, dado por (2.17), possui as seguintes propriedades

- $E\left(\mathrm{I}^{\mathrm{T}}\left(\omega_{\mathrm{k}}\right)\right)=\mathrm{f}_{\mathrm{x}}\left(\omega_{\mathrm{k}}\right)+\mathrm{O}\left(\mathrm{T}^{-1}\right)$; 
- $\operatorname{Var}\left(\mathrm{I}^{\mathrm{T}}\left(\omega_{\mathrm{k}}\right)\right)=\mathrm{f}_{\mathrm{x}}^{2}\left(\omega_{\mathrm{k}}\right)+\mathrm{O}\left(\mathrm{T}^{-1}\right)$

- $\frac{2 \mathrm{I}^{\mathrm{T}}\left(\omega_{\mathrm{k}}\right)}{\mathrm{f}_{\mathrm{x}}\left(\omega_{\mathrm{k}}\right)} \underset{\mathrm{T} \rightarrow \infty}{\stackrel{D}{\longrightarrow}} \chi_{2}^{2}$

Definição 2.11: Seja $\left\{X_{t}, t=0,1, \ldots, T-1\right\}$ uma série temporal estacionária. $O$ periodograma suavizado é dado por

$$
\hat{f}_{x}^{s}(\lambda)=\frac{2 \pi}{T} \sum_{s=1}^{T-1} W^{T}\left(\lambda-\omega_{s}\right) I^{T}\left(\omega_{s}\right),-\infty<\lambda<\infty
$$

em que $\omega_{\mathrm{s}}=\frac{2 \pi \mathrm{s}}{\mathrm{T}},-\infty<\omega_{\mathrm{s}}<\infty, \mathrm{W}^{\mathrm{T}}(\alpha),-\infty<\alpha<\infty, \mathrm{T}=1,2, \ldots$ é uma família de funções peso de período $2 \pi$ definida de tal forma que a expressão (2.18) envolve uma ponderação de $\left(2 \mathrm{~m}_{\mathrm{T}}+1\right)$ ordenadas na vizinhança da freqüência $\lambda, \mathrm{m}_{\mathrm{T}}$ satisfaz as seguintes restrições: $\mathrm{m}_{\mathrm{T}} \underset{\mathrm{T} \rightarrow \infty}{\longrightarrow} \infty$ e $\frac{\mathrm{m}_{\mathrm{T}}}{\mathrm{T}} \underset{\mathrm{T} \rightarrow \infty}{\longrightarrow} 0$.

Uma maneira conveniente de construir a função peso $\mathrm{W}^{\mathrm{T}}$ é considerar a seqüência de parâmetros $\mathrm{B}_{T}, \mathrm{~T}=1,2, \ldots$, satisfazendo $\mathrm{B}_{T}>0, \mathrm{~B}_{\mathrm{T}} \rightarrow 0, \mathrm{~B}_{\mathrm{T}} \mathrm{T} \rightarrow \infty$ quando $\mathrm{T} \rightarrow \infty$ e considerando

$$
\mathrm{W}^{\mathrm{T}}(\alpha)=\sum_{\mathrm{j}=-\infty}^{\infty} \mathrm{B}_{\mathrm{T}}^{-1} \mathrm{~W}\left(\mathrm{~B}_{\mathrm{T}}^{-1}(\alpha+2 \pi \mathrm{j})\right)-\infty<\alpha<\infty,
$$

em que $\mathrm{W}(\beta),-\infty<\beta<\infty$ é uma função peso que satisfaz as seguintes propriedades:

- $\int_{-\infty}^{\infty} \mathrm{W}(\beta) \mathrm{d} \beta=1$ 
$\int_{-\infty}^{\infty}|\mathrm{W}(\beta)| \mathrm{d} \beta<\infty$

Definição 2.12: Seja $\left\{X_{t}, t=0,1,2, . ., T-1\right\}$ uma série temporal estacionária, o estimador suavizado de covariâncias da função densidade espectral é dado por

$$
\hat{\mathrm{f}}_{\mathrm{x}}\left(\omega_{1}\right)=\frac{1}{2 \pi} \sum_{\mathrm{s}=-\infty}^{\infty} \lambda_{\mathrm{M}}(\mathrm{s}) \hat{\gamma}(\mathrm{s}) \cos \left(\mathrm{s} \omega_{1}\right)
$$

em que $\hat{\gamma}(s)=\frac{1}{T} \sum_{t=0}^{T-s-1}\left(X_{t}-\bar{X}\right)\left(X_{t+s}-\bar{X}\right), \quad \bar{X}=\frac{1}{T} \sum_{t=0}^{T-1} X_{t}$ e $\lambda_{M}($.$) é uma função$ peso, denominada núcleo, que satisfaz as condições seguintes:

- $0 \leq \lambda_{\mathrm{M}}(\mathrm{s}) \leq \lambda_{\mathrm{M}}(0) \leq 1$;

- $\lambda_{M}(-s) \leq \lambda_{M}(s)$, para todo $s ;$

- $\lambda_{\mathrm{M}}(\mathrm{s})=0$, para $|\mathrm{s}|>\mathrm{M}$.

Na Tabela 2.1 estão alguns exemplos de núcleos que podem ser utilizados no cálculo do estimador da função densidade espectral dado por (2.19), em que $\lambda_{\mathrm{M}}(\mathrm{s})=\mathrm{k}\left(\frac{\mathrm{s}}{\mathrm{M}}\right)$. 
Tabela 2.1 - Geradores de "lag window"

\begin{tabular}{|l|l|}
\hline Daniel & $\mathrm{k}(\mathrm{u})=\frac{\operatorname{sen}(\mathrm{u} \pi)}{(\mathrm{u} \pi)}$ \\
\hline Tukey-Hamming & $\mathrm{k}(\mathrm{u})= \begin{cases}0,54+0,46 \cos (\mathrm{u} \pi) \quad|\mathrm{u}| \leq 1 \\
0 & \text { c.c. }\end{cases}$ \\
\hline Bartlett-Priestley & $\mathrm{k}(\mathrm{u})=\frac{3}{(\mathrm{u} \pi)^{2}}\left(\frac{\operatorname{sen}(\mathrm{u} \pi)}{\mathrm{u} \pi}-\cos (\mathrm{u} \pi)\right)$ \\
\hline Parzen & $\mathrm{k}(\mathrm{u})= \begin{cases}1-6 \mathrm{u}^{2}+6|\mathrm{u}|^{3} & |\mathrm{u}| \leq \frac{1}{2} \\
2(1-|\mathrm{u}|)^{3} & \frac{1}{2} \leq|\mathrm{u}| \leq 1 \\
0 & \text { c.c. }\end{cases}$ \\
\hline
\end{tabular}

\section{Resultado}

Pode-se demonstrar que, os estimadores periodograma suavizado, dado por (2.18), e suavizado de covariâncias, dado por (2.19), são assintoticamente equivalentes se $\mathrm{W}^{\mathrm{T}}(\alpha)=\frac{1}{2 \pi} \sum_{\tau} \exp (-\mathrm{i} \alpha \tau) \lambda_{\mathrm{M}}(\tau)$.

\section{Propriedades}

- $E\left(\hat{f}_{x}^{s}\left(\omega_{k}\right)\right)=f_{x}\left(\omega_{k}\right)+O\left(T^{-1}\right)$; 


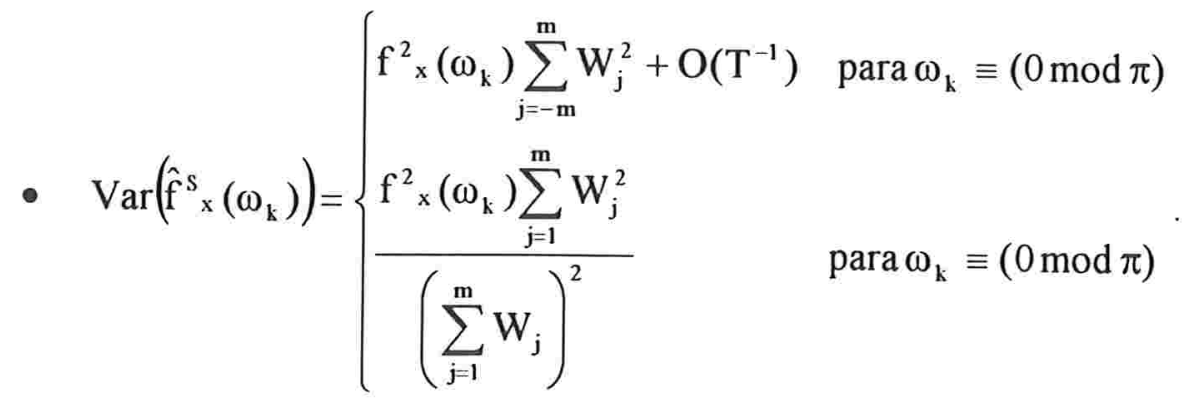

Definição 2.13: Seja $\left\{\mathrm{X}_{\mathrm{t}}, \mathrm{t}=0,1,2, . ., \mathrm{T}-1\right\}$ uma série temporal estacionária, o estimador suavizado de bi-covariâncias da função densidade bi-espectral é dado por

$$
\hat{f}_{x}\left(\omega_{1}, \omega_{2}\right)=\frac{1}{(2 \pi)^{2}} \sum_{s_{1}=-(T-1)}^{(T-1)} \sum_{s_{2}=-(T-1)}^{(T-1)} \lambda\left(\frac{s_{1}}{M}, \frac{s_{2}}{M}\right) \hat{C}\left(s_{1}, s_{2}\right) \exp \left(- \text { is }_{1} \omega_{1}-\text { is }_{2} \omega_{2}\right)
$$

em que $\hat{\mathrm{C}}\left(\mathrm{s}_{1}, \mathrm{~s}_{2}\right)=\frac{1}{\mathrm{~T}} \sum_{\mathrm{t}=0}^{\mathrm{T}-1-\gamma}\left(\mathrm{X}_{\mathrm{t}}-\overline{\mathrm{X}}\right)\left(\mathrm{X}_{\mathrm{t}+\mathrm{s}_{1}}-\overline{\mathrm{X}}\right)\left(\mathrm{X}_{\mathrm{t}+\mathrm{s}_{2}}-\overline{\mathrm{X}}\right)$ para $\mathrm{s}_{1}, \mathrm{~s}_{2} \geq 0, \gamma=\max \left(0, \mathrm{~s}_{1}, \mathrm{~s}_{2}\right)$,
$\bar{X}=\frac{1}{\mathrm{~T}} \sum_{\mathrm{t}=0}^{\mathrm{T}-1} \mathrm{X}_{\mathrm{t}}$ e $\lambda(.,$.$) é uma função peso, com dois argumentos, denominada núcleo$ que satisfaz as seguintes condições

$\lambda(t, s)=\lambda(s, t)=\lambda(-t, s-t)=\lambda(s-t,-t)$

- $\lambda(\mathrm{t}, \mathrm{s})=\lambda_{\mathrm{M}}(\mathrm{t}) \lambda_{\mathrm{M}}(\mathrm{s}) \lambda_{\mathrm{M}}(\mathrm{t}-\mathrm{s})$.

Definição 2.14: Seja $\left\{X_{t}, t=0, \pm 1, \pm 2, \ldots\right\}$ uma série temporal estacionária. A bicoerência nas freqüências $\left(\omega_{1}, \omega_{2}\right)$ é dada por 


$$
\gamma^{2}\left(\omega_{1}, \omega_{2}\right)=\frac{\left|f_{x}\left(\omega_{1}, \omega_{2}\right)\right|^{2}}{f_{x}\left(\omega_{1}\right) f_{x}\left(\omega_{2}\right) f_{x}\left(\omega_{1}+\omega_{2}\right)},
$$

para $0 \leq \omega_{2} \leq \omega_{1}$ e $\omega_{1}+\frac{\omega_{2}}{2} \leq \pi$.

Definição 2.15: Seja $\left\{X_{t}, t=0,1,2, . ., T-1\right\}$ uma série temporal estacionária, um estimador da bi-coerência é dado por

$$
\hat{\gamma}^{2}\left(\omega_{1}, \omega_{2}\right)=\frac{\left|\hat{\mathrm{f}}_{\mathrm{x}}\left(\omega_{1}, \omega_{2}\right)\right|^{2}}{\hat{\mathrm{f}}_{\mathrm{x}}\left(\omega_{1}\right) \hat{\mathrm{f}}_{\mathrm{x}}\left(\omega_{2}\right) \hat{\mathrm{f}}_{\mathrm{x}}\left(\omega_{1}+\omega_{2}\right)}
$$

para $0 \leq \omega_{2} \leq \omega_{1}$ e $\omega_{1}+\frac{\omega_{2}}{2} \leq \pi$, em que $\hat{f}_{x}\left(\omega_{1}\right)$ e $\hat{f}_{x}\left(\omega_{1}, \omega_{2}\right)$ são dados pelas expressões (2.19) e (2.20), respectivamente.

Definição 2.16: $O$ processo $X_{t}$ será uma diferença martingala quando satisfizer as condições

i) $\mathrm{E}\left|\mathrm{X}_{\mathrm{t}}\right|<\infty$ para todo $\mathrm{t}$;

ii) $E\left(X_{t} / f\left(X_{t-1}, X_{t-2}, \ldots\right)\right)=0$ para todo $t$

Todas as diferenças martingalas possuem média zero e são serialmente nãocorrelacionadas. Uma diferença martingala impõe uma condição mais forte do que não-autocorrelação. Uma seqüência serialmente não-correlacionada não pode ser prevista com base em uma função linear de seus valores passados. No caso de uma diferença martingala, nenhuma seqüência de valores passados, linear ou não-linear, pode prevê-la. 


\section{Capítulo 3}

\section{Modelos Lineares em Séries Temporais}

\subsection{Introdução}

Quando modelamos uma série temporal $X_{t}\{t=0, \pm 1, \pm 2, \ldots\}$ queremos obter uma relação entre ..., $X_{t-2}, X_{t-1}, X_{t}, X_{t+1}, X_{t+2}, \ldots$ que resulte em um processo ruído branco $e_{t}$. Desta forma um modelo geral para $X_{t}$ pode ser dado por

$$
h\left(\ldots, X_{t-2}, X_{t-1}, X_{t}, X_{t+1}, X_{t+2}, \ldots\right)=e_{t}
$$

Quando a função $\mathrm{h}\left(\right.$.) é linear temos um modelo linear para $\mathrm{X}_{\mathrm{t}}$ e a expressão (3.1) pode ser reescrita como

$$
\sum_{u=-\infty}^{\infty} h_{u} X_{t-u}=e_{t},
$$

em que $\left\{h_{u}\right\}$ é uma seqüência de constantes. 
A expressão (3.2) mostra a forma geral de um modelo linear para a série $X_{t}$, levando em conta que $\mathrm{X}_{\mathrm{t}}$ depende de valores passados, presente e futuros. Considerando que $X_{t}$ dependa somente de valores passados temos que

$$
\sum_{u=0}^{\infty} h_{u} X_{t-u}=e_{t}
$$

Podemos reescrever a expressão (3.3) na seguinte forma

$$
\mathrm{H}(\mathrm{B}) \mathrm{X}_{\mathrm{t}}=\mathrm{e}_{\mathrm{t}} \text {, }
$$

em que $H(B)=\sum_{u=0}^{\infty} h_{u} B^{u}$ e $B^{m} X_{t}=X_{t-m}$

Se as raízes de $\mathrm{H}(\mathrm{B})=0$ forem, em módulo, maiores que um podemos inverter a expressão (3.4) e obter

$$
X_{t}=H^{-1}(B) e_{t}=G(B) e_{t}
$$

em que $G(B)=g_{0}+g_{1} B+g_{2} B^{2}+\ldots$

Desta forma a série $X_{t}$ pode ser representada pela expressão

$$
X_{t}=\sum_{u=0}^{\infty} g_{u} e_{t-u} .
$$

Se considerarmos e um processo de entrada e $X_{t}$ um processo de saída, a função $\mathrm{G}(\mathrm{B})$ é chamada de função de transferência do modelo. 
3.2 Modelo auto-regressivo de médias móveis

Definição 3.1: Uma série temporal $X_{t}\{t=0, \pm 1, \pm 2, \ldots\}$ segue um modelo autoregressivo de médias móveis de ordem $\mathrm{p}, \mathrm{q}, \operatorname{ARMA}(\mathrm{p}, \mathrm{q})$, se satisfizer a equação

$$
X_{t}=\phi_{1} X_{t-1}+\phi_{2} X_{t-2}+\ldots+\phi_{p} X_{t-p}+e_{t}-\theta_{1} e_{t-1}-\theta_{2} e_{t-2}-\ldots-\theta_{q} e_{t-q},
$$

em que

$\mathrm{e}_{\mathrm{t}}$ é um processo ruído branco com média zero e variância $\sigma_{\mathrm{e}}^{2}$ constante; $\phi_{1}, \phi_{2}, \ldots, \phi_{\mathrm{p}}, \theta_{1}, \theta_{2}, \ldots, \theta_{\mathrm{q}}$ são os parâmetros do modelo.

A expressão (3.7) pode ser reescrita na forma reduzida

$$
\phi(B) X_{t}=\theta(B) e_{t}
$$

em que

$\phi(B)=\left(1-\phi_{1} B-\phi_{2} B^{2}-\ldots-\phi_{p} B^{p}\right)$ é o operador auto-regressivo de ordem $p$; $\theta(B)=\left(1-\theta_{1} B-\theta_{2} B^{2}-\ldots-\theta_{q} B^{q}\right)$ é o operador de médias móveis de ordem $q$; $\mathrm{B}^{\mathrm{m}} \mathrm{X}_{\mathrm{t}}=\mathrm{X}_{\mathrm{t}-\mathrm{m}}$.

O modelo (3.8) será estacionário se a série $\theta(\mathrm{B})$ convergir para $|\mathrm{B}| \leq 1$ e será invertível se $\phi(B)$ convergir para $|\mathrm{B}| \leq 1$, ou seja, o modelo será estacionário se as raizes de $\phi(B)=0$ forem, em módulo, maiores que um e será invertível se as raízes de $\theta(B)=0$ forem, em módulo, maiores que um. Para maiores detalhes, ver Box, Jenkins e Reinsel (1994).

Se na expressão (3.7), $\phi_{\mathrm{j}}=0$ para $\mathrm{j}>\mathrm{p}$ e $\theta_{\mathrm{q}}=0$ para todo $\mathrm{q}$, obteremos o modelo auto-regressivo de ordem $p$, que denotaremos por $\mathrm{AR}(\mathrm{p})$ 


$$
\mathrm{X}_{\mathrm{t}}=\phi_{1} \mathrm{X}_{\mathrm{t}-1}+\phi_{2} \mathrm{X}_{\mathrm{t}-2}+\ldots+\phi_{\mathrm{p}} \mathrm{X}_{\mathrm{t}-\mathrm{p}}+\mathrm{e}_{\mathrm{t}}
$$

ou

$$
\phi(B) \mathrm{X}_{\mathrm{t}}=\mathrm{e}_{\mathrm{t}}
$$

O modelo (3.10) será estacionário se a série $\phi(B)$ convergir para $|\mathrm{B}| \leq 1$ e será sempre invertivel, ou seja, o modelo será estacionário e invertivel se as raízes de $\phi(B)=0$ forem em módulo maiores que um.

Se na expressão (3.7), $\theta_{\mathrm{j}}=0$ para $\mathrm{j}>\mathrm{q}$ e $\phi_{\mathrm{p}}=0$ para todo $\mathrm{p}$, obteremos o modelo de médias móveis de ordem q, que denotaremos por MA(q)

$$
X_{t}=e_{t}-\theta_{1} e_{1-1}-\theta_{2} e_{t-2}-\ldots-\theta_{q} e_{1-q}
$$

ou

$$
X_{t}=\theta(B) e_{1}
$$

O modelo (3.12) será sempre estacionário e será invertível se a série $\theta(B)$ convergir para $|\mathrm{B}| \leq 1$, ou seja, o modelo será estacionário e invertivel se as raízes $\operatorname{de} \theta(B)=0$ forem em módulo maiores que um.

\subsection{Extensões do modelo auto-regressivo de médias móveis}

Existem alguns tipos de séries temporais que são não estacionárias mas tornam-se estacionárias ao aplicarmos sucessivas diferenças à série original. Esta é uma característica do modelo auto-regressivo integrado de médias móveis. Algumas séries temporais apresentam componentes periódicas que se repetem com período S e esta característica é incorporada pelo modelo sazonal auto-regressivo de médias móveis. $\mathrm{O}$ modelo que incorporada as características de não estacionariedade e sazonalidade de uma série temporal é chamado de modelo auto- 
regressivo integrado médias móveis sazonal, que denotaremos por $\operatorname{SARIMA}(\mathrm{p}, \mathrm{d}, \mathrm{q}) \mathrm{x}(\mathrm{P}, \mathrm{D}, \mathrm{Q})_{\mathrm{s}}$ e é dado por

$$
\Phi_{\mathrm{P}}\left(\mathrm{B}^{\mathrm{S}}\right) \phi(\mathrm{B}) \Delta_{\mathrm{S}}^{\mathrm{D}} \Delta^{\mathrm{d}} \mathrm{X}_{\mathrm{t}}=\Theta_{\mathrm{Q}}\left(\mathrm{B}^{\mathrm{S}}\right) \theta(\mathrm{B}) \mathrm{e}_{\mathrm{t}}
$$

em que

$\Phi_{\mathrm{P}}\left(\mathrm{B}^{\mathrm{S}}\right)=\left(1-\phi_{1} \mathrm{~B}^{\mathrm{S}}-\phi_{2} \mathrm{~B}^{2 \mathrm{~S}}-\ldots-\phi_{\mathrm{P}} \mathrm{B}^{\mathrm{PS}}\right)$ é o operador auto-regressivo sazonal de ordem $\mathrm{P}$; $\Theta_{Q}\left(B^{S}\right)=\left(1-\theta_{1} B^{S}-\theta_{2} B^{2 S}-\ldots-\theta_{Q} B^{Q S}\right)$ é o operador de médias móveis sazonal de ordem Q;

$\phi(B)=\left(1-\phi_{1} B-\phi_{2} B^{2}-\ldots-\phi_{p} B^{p}\right)$ é o operador auto-regressivo de ordem $p$; $\theta(B)=\left(1-\theta_{1} B-\theta_{2} B^{2}-\ldots-\theta_{q} B^{q}\right)$ é o operador de médias móveis de ordem $q$; $\Delta^{\mathrm{d}}=(1-\mathrm{B})^{\mathrm{d}}$ e $\Delta_{\mathrm{S}}^{\mathrm{D}}=\left(1-\mathrm{B}^{\mathrm{S}}\right)^{\mathrm{D}}$.

\subsection{Modelo linear e estacionariedade}

Segundo Priestley (1988), uma representação linear de uma série temporal estacionária $X_{t}$ pode sempre ser construída, desde que $X_{t}$ tenha espectro contínuo com função densidade espectral $f_{x}(\omega)$. Neste caso a série $X_{t}$ pode ser escrita na forma

$$
X_{t}=\sum_{u=-\infty}^{\infty} h_{u} \varepsilon_{t-u}
$$

em que $\varepsilon_{t}$ é uma seqüência de variáveis aleatórias não correlacionadas.

Se $f_{x}(\omega)$ satisfizer $\int_{-\pi}^{\pi} \log \left(f_{x}(\omega)\right) d \omega>-\infty$, podemos substituir a expressão (3.14) pela expressão 


$$
X_{1}=\sum_{u=0}^{\infty} h_{u} \varepsilon_{t-u} .
$$

Devemos notar que a representação linear obtida na expressão (3.15) é diferente da representação dada pela expressão (3.6) porque na expressão (3.6), a é uma seqüência de variáveis aleatórias independentes e o processo $\varepsilon_{\mathrm{t}}$ da expressão (3.15), é uma seqüência de variáveis aleatórias não correlacionadas. Quando $X_{t}$ é um processo gaussiano então $\varepsilon_{t}$ é gaussiano e, por ser não correlacionado, é independente. Neste caso especial a distinção entre $\varepsilon_{t}$ e e desaparece e a série $X_{t}$ pode ser escrita como

$$
X_{1}=\sum_{u=0}^{\infty} h_{u} e_{t-u}
$$




\section{Capítulo 4}

\section{Alguns Modelos Não-Lineares em Séries Temporais}

\subsection{Introdução}

No Capítulo 3 foi apresentada a forma geral dos modelos lineares de séries temporais. Quando $\mathrm{X}_{\mathrm{t}}$ depende apenas dos valores passados temos que

$$
h\left(X_{t}, X_{t-1}, X_{t-2}, \ldots\right)=e_{t}
$$

Vamos supor que $\mathrm{h}($.) é tal que o modelo dado pela expressão (4.1) é invertível. Desta forma podemos escrever a expressão (4.1) como

$$
X_{t}=h^{-1}\left(e_{t}, e_{t-1}, e_{t-2, \ldots)}\right.
$$


Assumindo que $\mathrm{h}^{-1}$ (.) seja uma função suficientemente bem comportada e que pode ser expandida em uma série de Taylor sobre o ponto $\mathrm{O}=(0,0,0, \ldots)$, então podemos expandir o lado direito de (4.2) e escrever

$$
X_{t}=\mu+\sum_{u=0}^{\infty} g_{u} e_{t-u}+\sum_{u=0}^{\infty} \sum_{v=0}^{\infty} g_{u v} e_{t-u} e_{t-v}+\sum_{u=0}^{\infty} \sum_{v=0}^{\infty} \sum_{w=0}^{\infty} g_{u v w} e_{t-u} e_{t-v} e_{t-w}+\ldots
$$

em que $\mu=h^{\prime}(0), g_{u}=\left(\frac{\partial h}{\partial e_{t-u}}\right)_{0}, g_{u v}=\left(\frac{\partial^{2} h}{\partial e_{t-u} \partial e_{t-v}}\right)_{0}$ e $g_{u v w}=\left(\frac{\partial^{3} h}{\partial e_{t-u} \partial e_{t-v} \partial e_{t-w}}\right)_{0}$.

A expansão dada pela expressão (4.3) é conhecida como série de Volterra e é uma forma importante de representação para modelos não-lineares.

\subsection{Algumas características importantes de sistemas não- lineares}

Os sistemas não-lineares podem apresentar algumas das seguintes características:

\section{Dependência amplitude-freqüiência}

Em alguns sistemas não-lineares a amplitude do processo é dependente da freqüência, por exemplo, a amplitude da série aumenta quando a freqüência aumenta. Este comportamento é chamado de dependência amplitude-freqüência. Na Figura 4.1 está representada uma série em que a amplitude é alta quando a freqüência é baixa e a amplitude é baixa quando a freqüência é alta. Na Figura 4.2 
temos um exemplo de série em que a amplitude é alta quando a freqüência é alta e a amplitude é baixa quando a freqüência é baixa.

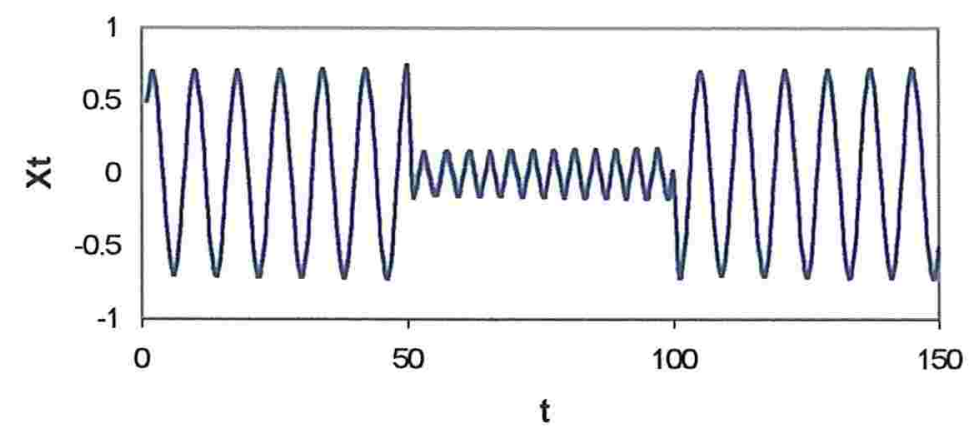

Figura 4.1 - Dependência amplitude-freqüência.

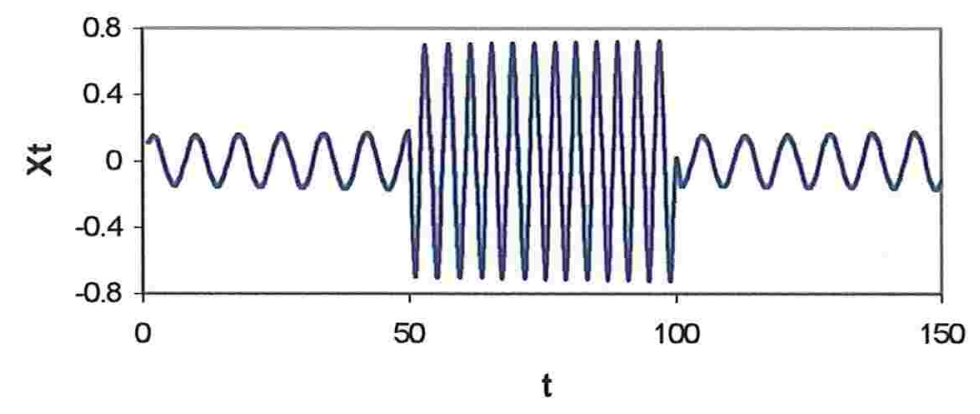

Figura 4.2 - Dependência amplitude-freqüência.

\section{Fenômeno de salto}

A amplitude de saída de um sistema não-linear, diferentemente de um sistema linear, pode ter "saltos" em diferentes freqüências dependendo se a freqüência de entrada, de amplitude constante, é monotonicamente crescente ou decrescente como mostram as Figuras 4.3 e 4.4. Este comportamento é chamado de fenômeno de salto. Na Figura 4.5 estão representadas a entrada e a saída de um modelo não-linear com amplitude de entrada constante, podemos notar que a amplitude de saída possui "saltos" em diferentes freqüências dependendo da freqüência de entrada. 
Capítulo 4 - Alguns modelos não-lineares em séries temporais

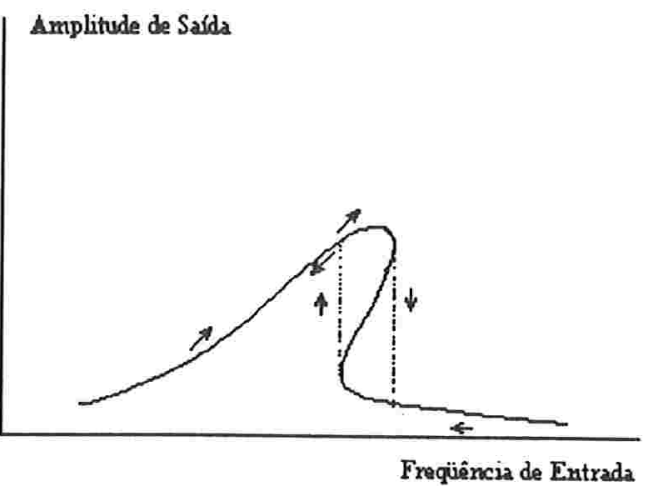

Figura 4.3 - Freqüência de entrada monotonicamente crescente.

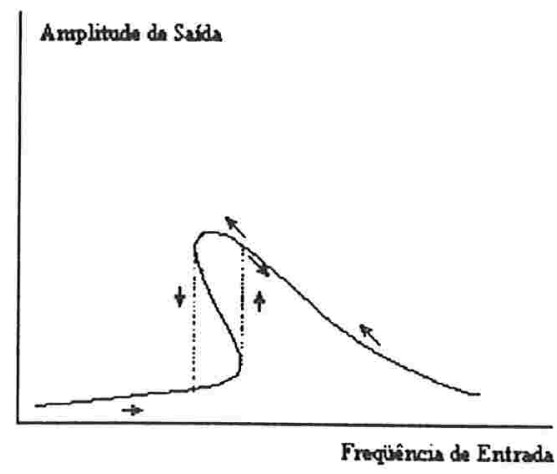

Figura 4.4 - Freqüência de entrada monotonicamente decrescente. 

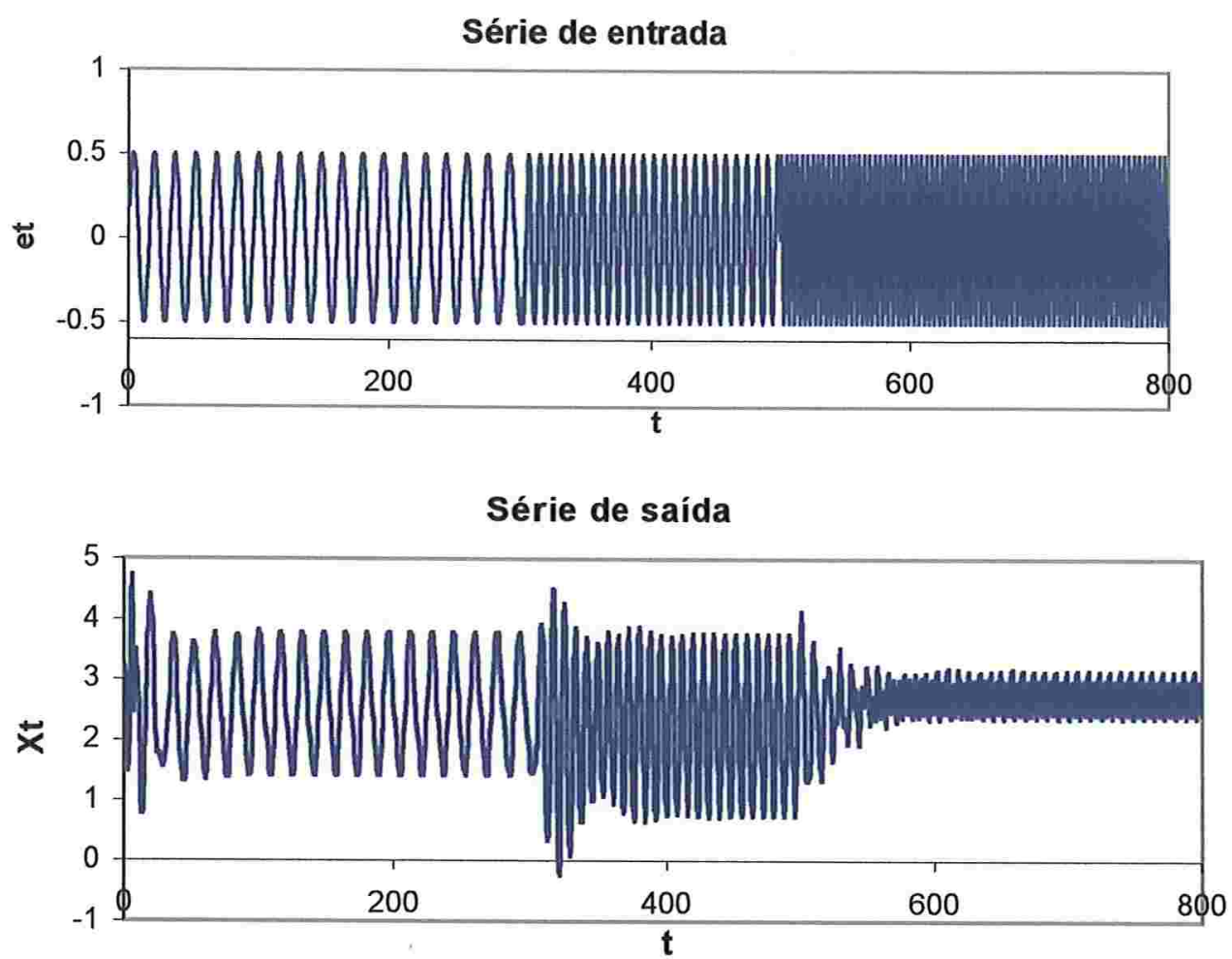

Figura 4.5 - Séries de entrada e saída de um sistema não-linear.

A amplitude de saída de um sistema linear pode também ter "saltos" em diferentes amplitudes dependendo se a amplitude de entrada, de freqüência constante, é monotonicamente crescente ou decrescente. Na Figura 4.6 está representada a série de entrada, com amplitude menor e a saída, com amplitude maior, de um modelo não-linear com freqüência de entrada constante.

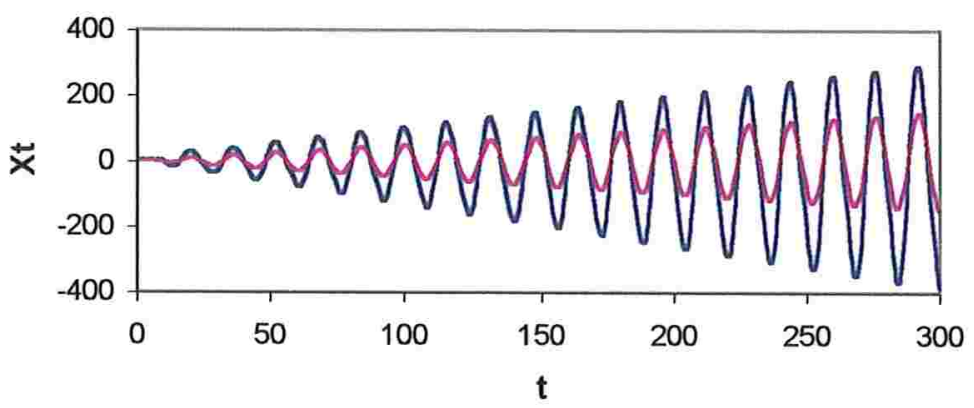

Figura 4.6 - Séries de entrada e saída. 


\section{Ciclos limites}

Este é um típico comportamento de séries que possuem periodicidades, o ciclo limite pode ser estável ou instável. Este comportamento pode ser detectado nos gráficos de $\left(X_{t} \times X_{t-1}\right)$ quando ele existe. Nas Figuras 4.7 e 4.8 estão representados os ciclos limites estáveis e instáveis respectivamente.

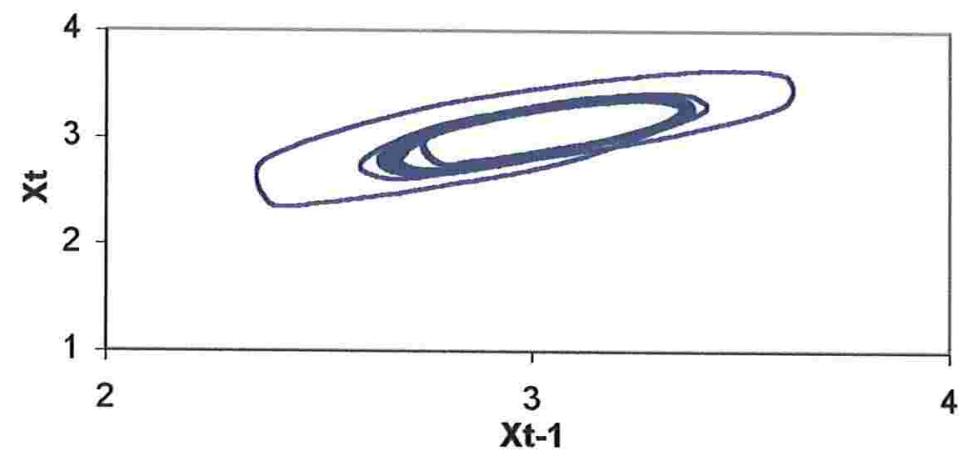

Figura 4.7 - Ciclo limite estável.

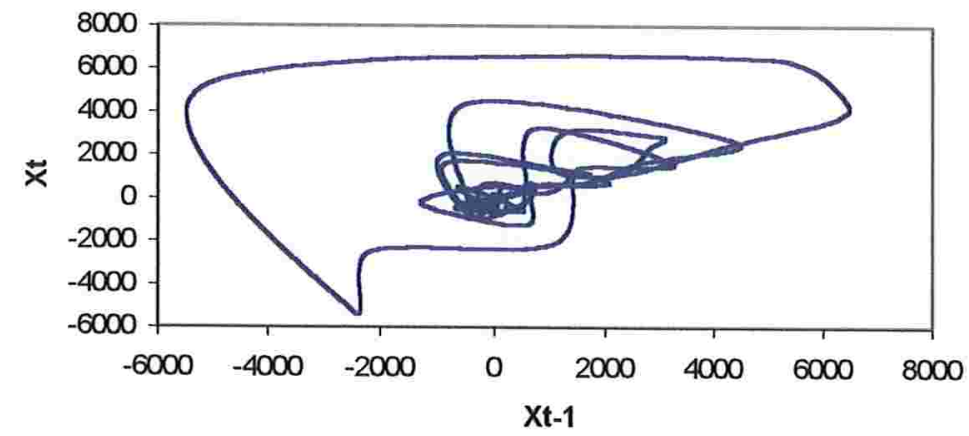

Figura 4.8 - Ciclo limite instável. 


\subsection{Alguns modelos não-lineares}

\subsubsection{Modelo auto-regressivo com limiar ("Threshold")}

A idéia essencial da classe dos modelos auto-regressivos com limiar é a linearização por partes dos modelos não-lineares, por meio da introdução de limiares. Estes modelos são localmente lineares e foram introduzidos por Tong (1977).

Definição 4.1: Uma série temporal $X_{t}\{t=0, \pm 1, \pm 2, \ldots\}$ segue um modelo autoregressivo com limiar, $\operatorname{SETAR}\left(\mathrm{q}, \mathrm{k}_{1}, \mathrm{k}_{2}, \ldots, \mathrm{k}_{\mathrm{q}}\right)$, se satisfizer a equação

$$
X_{t}=a_{0}^{(j)}+\sum_{i=1}^{k_{j}} a_{i}^{(j)} X_{t-i}+e_{t}^{(j)}, \text { se } X_{t-d} \in R_{j} \text { para } j=1,2, \ldots, q
$$

em que

$\mathrm{e}_{\mathrm{t}}^{(\mathrm{j})}$ são variáveis aleatórias independentes e identicamente distribuídas com média zero;

$R_{j}$ é um intervalo da reta $I R$ tal que $R_{1} \cup R_{2} \cup \ldots \cup R_{q}=1 R ; \quad R_{i}=\left(r_{i-1}, r_{i}\right)$ para $-\infty<\mathrm{r}_{0}<\ldots<\mathrm{r}_{\mathrm{q}}<+\infty$;

$\mathrm{r}_{0, \ldots, \mathrm{r}_{\mathrm{q}-1}}$ denotam os parâmetros de limiar;

$\mathrm{k}_{\mathrm{j}}$ denota a ordem da j-ésima auto-regressão;

d é o parâmetro de retardo do processo;

$\mathrm{a}_{\mathrm{i}}^{(j)}$ são constantes;

q é o número de regimes do processo.

Podemos verificar que o modelo $\operatorname{SETAR}(1 ; \mathrm{k})$ é um modelo auto-regressivo de ordem $\mathrm{k}$. 
Nas Figuras 4.9 e 4.10 apresentamos 1000 observações geradas pelos modelos

(i) $X_{t}=\left\{\begin{array}{lll}0,4-0,8 X_{t-1}+0,3 X_{t-2}-0,7 X_{t-3}+e_{t} & \text { se } & X_{t-1}<2,8 \\ 0,2+0,9 X_{t-1}+0,4 X_{t-2}-0,6 X_{t-3}+e_{t} & \text { se } & X_{t-1}>2,8\end{array}\right.$

(ii) $X_{t}=\left\{\begin{array}{lll}0,15+0,85 X_{t-1}+0,22 X_{t-2}-0,7 X_{t-3}+e_{t} & \text { se } & X_{t-1}<1 \\ 0,30-0,90 X_{t-1}+0,30 X_{t-2}-0,7 X_{t-3}+e_{t} & \text { se } & X_{t-1}>1\end{array}\right.$

em que $e_{t} \sim N\left(0,(0,003)^{2}\right)$ independentes.

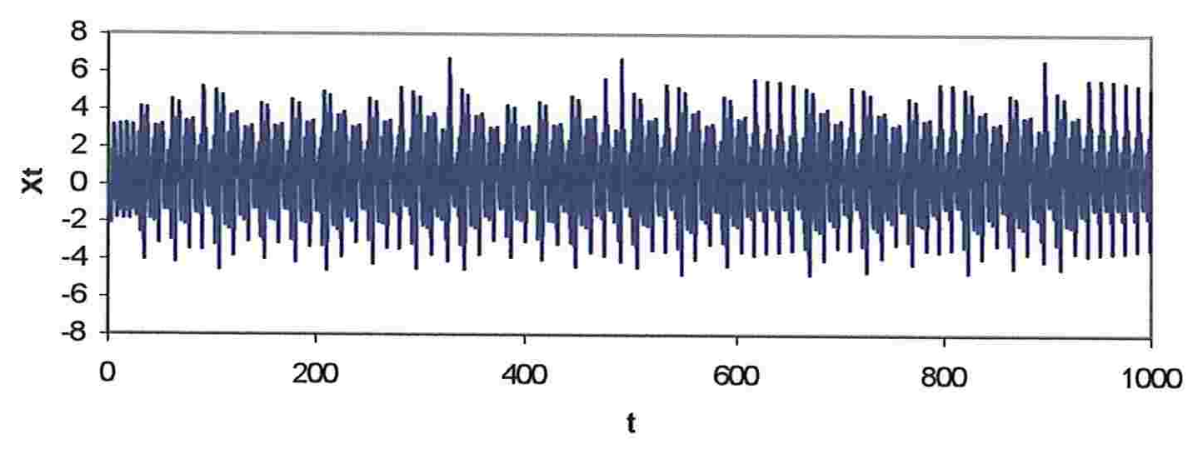

Figura 4.9 - Série gerada pelo modelo (4.5).

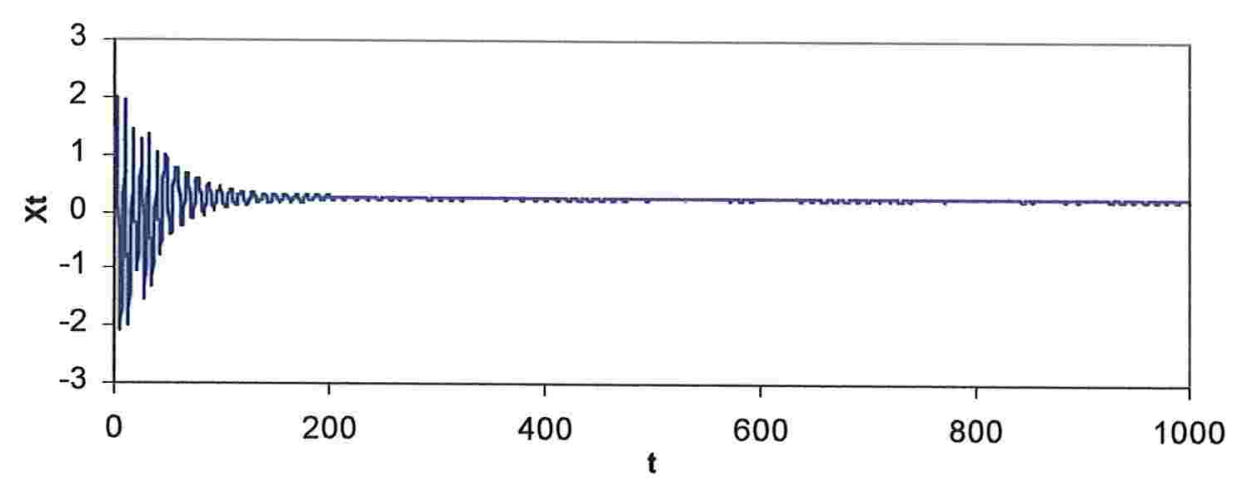

Figura 4.10 - Série gerada pelo modelo (4.6). 
Segundo Priestley (1988) o modelo auto-regressivo com limiar possui as seguintes características:

- dependência amplitude-freqüência;

- fenômeno de salto;

- ciclos limites.

\subsubsection{Modelo bilinear}

Os modelos bilineares foram introduzidos por Mohler (1973) e Ruberti et al (1972) e na literatura estatística foram introduzidos por Granger e Andersen (1978). Um estudo mais sistemático foi feito por Subba Rao (1981) e por Subba Rao e Gabr (1984).

Definição 4.2: Uma série temporal $\mathrm{X}_{\mathrm{t}}\{\mathrm{t}=0, \pm 1, \pm 2, \ldots\}$ segue um modelo bilinear, $\mathrm{BL}(\mathrm{p}, \mathrm{r}, \mathrm{m}, \mathrm{k})$, se satisfizer a equação

$$
X_{t}=\sum_{j=1}^{p} a_{j} X_{t-j}+\sum_{j=0}^{r} c_{j} e_{t-j}+\sum_{i=1}^{m} \sum_{j=1}^{k} b_{i j} X_{t-i} e_{t-j},
$$

em que

$a_{j}, j=1, \ldots, p, c_{j}, j=1 \ldots, r, b_{i j}$ são constantes sendo $c_{0}=1$;

$\mathrm{e}_{\mathrm{t}}$ é um processo ruído branco com média zero.

O modelo bilinear é uma extensão do modelo linear ARMA dado pela expressão (3.7), pois se $b_{i j}=0$ para todo $i, j$, então (4.7) se reduz a (3.7). 
Nas Figuras 4.11, 4.12 e 4.13 apresentamos 1000 observações geradas pelos modelos bilineares

(iii) $\mathrm{X}_{\mathrm{t}}=0,3 \mathrm{X}_{\mathrm{t}-1}+0,3 \mathrm{X}_{\mathrm{t}-1} \mathrm{e}_{\mathrm{t}-1}+\mathrm{e}_{\mathrm{t}}$

(iv) $X_{t}=0,3 X_{t-1}+0,8 X_{t-1} e_{t-1}+e_{t}$

(v) $X_{t}=0,8 X_{t-1}-0,3 X_{t-2}+0,5 X_{t-1} e_{t-1}+0,7 X_{t-2} e_{t-1}+e_{t}$

com $\mathrm{e}_{\mathrm{t}} \sim \mathrm{N}(0,1)$ independentes.

Notamos pelas Figuras 4.11, 4.12 e 4.13 que a série gerada pelo modelo (4.8), que possui um pequeno coeficiente associado ao termo bilinear, possui uma forma razoavelmente convencional; a série dada pelo modelo (4.9), que possui um grande coeficiente associado ao termo bilinear, apresenta um número de explosões de grande amplitude e a série dada pelo modelo (4.10), que apresenta um adicional termo bilinear, apresenta uma extrema forma deste efeito. Estes comportamentos são características de certos dados sismológicos e particularmente de séries de terremotos e explosões subterrâneas.

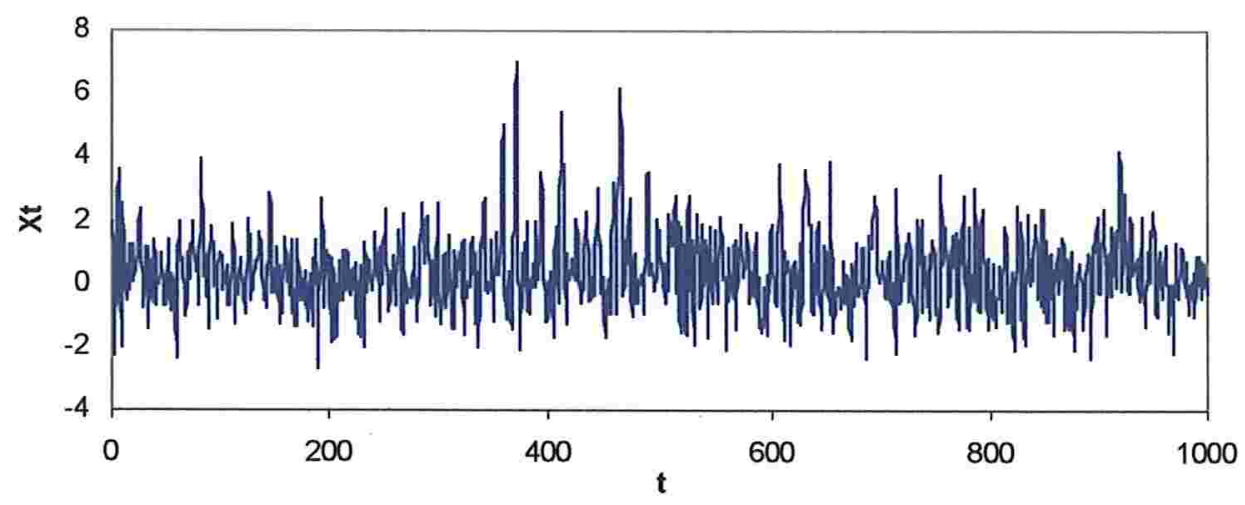

Figura 4.11 - Série gerada pelo modelo (4.8). 


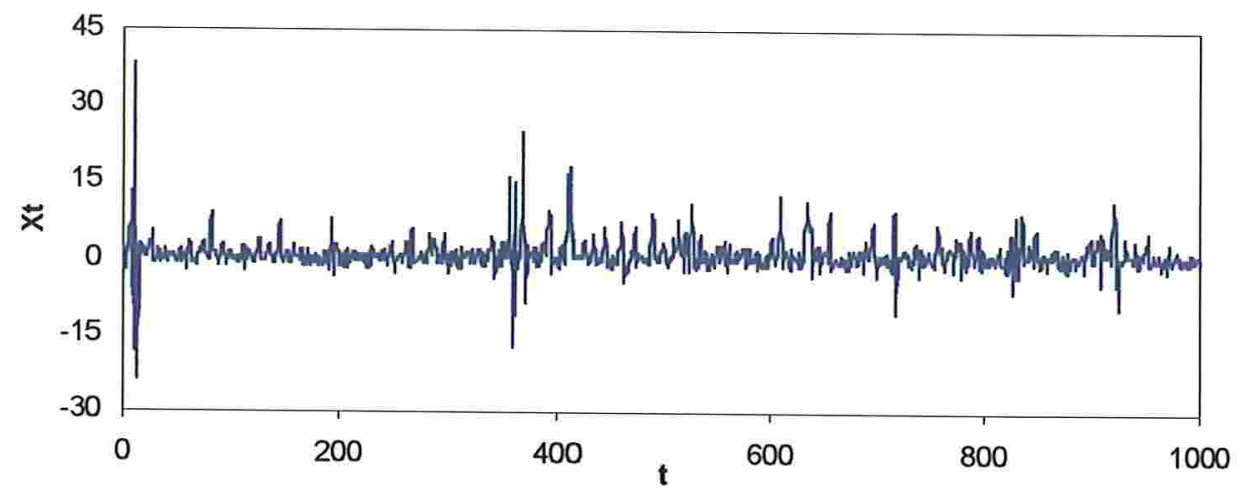

Figura 4.12 - Série gerada pelo modelo (4.9).

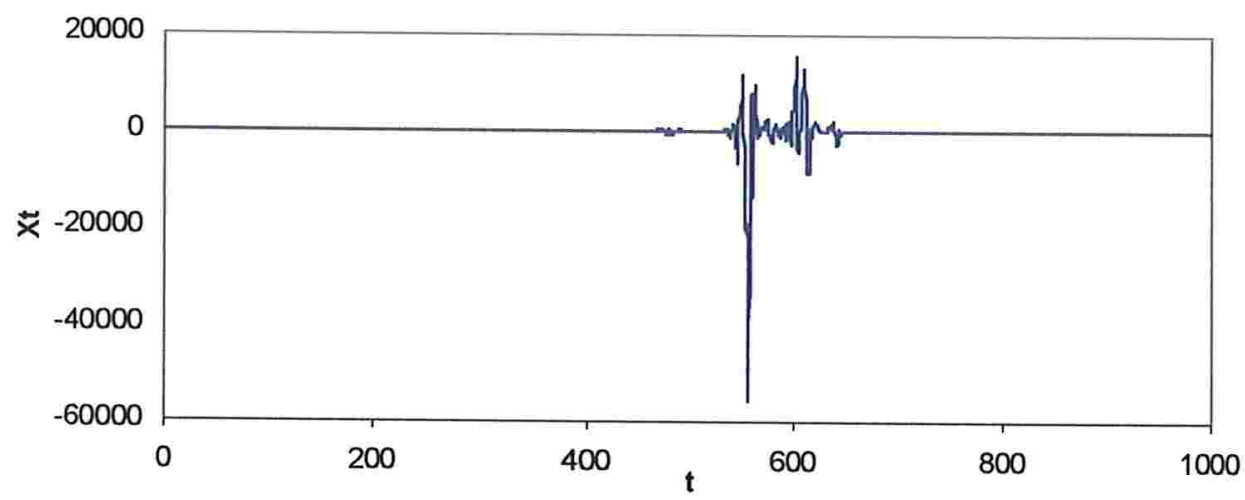

Figura 4.13 - Série gerada pelo modelo (4.10).

\section{Algumas propriedades do modelo bilinear}

Para obtermos as condições de estacionariedade consideraremos o modelo bilinear BL(p,0,p,1) dado por

$$
X_{t}+\sum_{j=1}^{p} a_{j} X_{t-j}=e_{t}+\sum_{i=1}^{p} b_{i 1} X_{t-i} e_{t-1}
$$

O modelo bilinear acima pode ser escrito na forma de espaço de estados com a seguinte representação 


$$
\begin{gathered}
\mathrm{X}_{\mathrm{t}}=\mathrm{Hx}_{\mathrm{t}} \\
\mathrm{x}_{\mathrm{t}}=\mathrm{Ax_{ \textrm {t } - 1 }}+\mathrm{Bx}_{\mathrm{t}-1} \mathrm{e}_{\mathrm{l}-1}+\mathrm{Ce}_{\mathrm{t}}
\end{gathered}
$$

em que

$$
\begin{aligned}
A_{p x p} & =\left[\begin{array}{cccc}
-a_{1} & -a_{2} & \cdots & -a_{p} \\
1 & 0 & \cdots & 0 \\
0 & 1 & \cdots & 0 \\
0 & 0 & 1 & 0
\end{array}\right], B_{p x p}=\left[\begin{array}{cccc}
b_{11} & b_{21} & \cdots & b_{p 1} \\
0 & 0 & \cdots & 0 \\
\cdots & \cdots & \cdots & \cdots \\
0 & 0 & \cdots & 0
\end{array}\right], C_{p x 1}=(1,0, \ldots, 0)^{\prime} \mathrm{e} \\
H_{1 x p} & =(1,0, \ldots, 0)
\end{aligned}
$$

As condições para estacionariedade assintótica são obtidas por Subba Rao (1981) que assume que os e são variáveis aleatórias independentes e identicamente distribuídas com distribuição $\mathrm{N}(0,1)$.

O processo é assintoticamente estacionário de primeira ordem se

$$
\max \left|\lambda_{\mathrm{i}}(\mathrm{A})\right|<1
$$

em que $\left\{\lambda_{\mathrm{i}}(\mathrm{A})\right\}$ é o conjunto dos autovalores da matriz $\mathrm{A}$.

Segundo Subba Rao (1981) a média assintótica do processo é dada por

$$
\mu=(\mathrm{I}-\mathrm{A})^{-1} \mathrm{BC}
$$

e o processo é assintoticamente estacionário de segunda ordem se

$$
\|\mathrm{A}\|^{2}+\|\mathrm{B}\|^{2}<1
$$


sendo que $\|\mathrm{A}\|^{2}$ denota o maior autovalor de $\mathrm{AA}^{\prime}$ e $\|\mathrm{B}\|^{2}$ denota o maior autovalor de BB'.

Assumindo que o processo $X_{t}$ é estacionário de primeira e segunda ordem e que $T$ é suficientemente grande temos que $C(s)=E\left(X_{t+s}-\mu\right)\left(X_{t}-\mu\right)^{\prime}$ é dada por

$$
\begin{aligned}
& \mathrm{C}(0)=\mathrm{AC}(0) \mathrm{A}^{\prime}+\mathrm{BC}(0) \mathrm{B}^{\prime}+\Delta_{2}, \\
& \mathrm{C}(1)=\mathrm{AC}(0)+\Delta_{3},
\end{aligned}
$$

$\mathrm{e}$

$$
\mathrm{C}(\mathrm{s})=\mathrm{AC}(\mathrm{s}-1)=\mathrm{A}^{\mathrm{s}-1} \mathrm{C}(1)(\mathrm{s}=2,3, \ldots),
$$

em que

$\mu_{\mathrm{t}}=\mu=\mathrm{E}\left(\mathrm{x}_{\mathrm{t}}\right)$

$\Delta_{2}=\mathrm{B} \mu \mu^{\prime} \mathrm{B}^{\prime}+\mathrm{A}^{\prime} \mu \mu^{\prime} \mathrm{A}^{\prime}+\mathrm{ASB}^{\prime}+\mathrm{BS}^{\prime} \mathrm{A}^{\prime}+2 \mathrm{BCC}^{\prime} \mathrm{B}^{\prime}+\mathrm{CC}^{\prime}-\mu \mu^{\prime} ;$

$\Delta_{3}=\mathrm{A} \mu \mu^{\prime}+\mathrm{BS}-\mu \mu^{\prime}$

Utilizando a expressão (4.14) temos que $\gamma(s)+a_{1} \gamma(s-1)+\ldots+a_{p} \gamma(s-p)=0$, para $\mathrm{s}>1$, em que $\gamma(\mathrm{s})=\operatorname{Cov}\left(\mathrm{X}_{\mathrm{t}+\mathrm{s}}, \mathrm{X}_{\mathrm{t}}\right)$. Estas equações são as mesmas equações de YuleWalker para os modelos $\operatorname{ARMA}(\mathrm{p}, 1)$ e deste modo podemos mostrar que o modelo bilinear $B L(p, 0, p, 1)$ apresenta a mesma estrutura de covariância assintótica do modelo $\operatorname{ARMA}(\mathrm{p}, 1)$.

O processo é invertível se $\mathrm{H}^{\prime} \mathrm{BE}\left(\mathrm{x}(\mathrm{s}) \mathrm{x}^{\prime}(\mathrm{s})\right) \mathrm{B}^{\prime} \mathrm{H}<\left(\mathrm{H}^{\prime} \mathrm{C}\right)^{2}$.

O modelo bilinear possui a característica de captar saltos na série como pode ser visto pelas Figuras 4.12 e 4.13 . 


\subsubsection{Modelo auto-regressivo exponencial}

Os modelos auto-regressivos exponenciais foram introduzidos por Ozaki (1978), Lawrance e Lewis (1980) e Haggan e Ozaki (1981).

Definição 4.3: Uma série temporal $\mathrm{X}_{\mathrm{t}}\{\mathrm{t}=0, \pm 1, \pm 2, \ldots\}$ segue um modelo autoregressivo exponencial de ordem $\mathrm{k}, \operatorname{EXPAR}(\mathrm{k})$, se satisfizer a equação

$$
\mathrm{X}_{\mathrm{t}}=\left(\phi_{1}+\pi_{1} \exp \left(-\gamma \mathrm{X}_{\mathrm{t}-1}^{2}\right)\right) \mathrm{X}_{\mathrm{t}-1}+\ldots+\left(\phi_{\mathrm{k}}+\pi_{\mathrm{k}} \exp \left(-\gamma \mathrm{X}_{\mathrm{t}-1}^{2}\right)\right) \mathrm{X}_{\mathrm{t}-\mathrm{k}}+\mathrm{e}_{\mathrm{t}},
$$

em que

$\gamma, \pi_{1}, \ldots, \pi_{\mathrm{k}}, \phi_{1}, \ldots \phi_{\mathrm{k}}$ são constantes sendo que $\gamma>0$;

$e_{t}$ são variáveis aleatórias independentes e identicamente distribuídas com média zero e variância finita.

O modelo auto-regressivo exponencial de ordem 2 pode ser representado de forma similar ao modelo auto-regressivo com limiar por meio da seguinte forma

$$
X_{t}=a_{1} X_{t-1}+a_{2} X_{t-2}+e_{t}
$$

em que $a_{1}=\phi_{1}+\pi_{1} \exp \left(-\gamma \mathrm{X}_{\mathrm{t}-1}^{2}\right)$ e $\mathrm{a}_{2}=\phi_{2}+\pi_{2} \exp \left(-\gamma \mathrm{X}_{\mathrm{t}-1}^{2}\right)$.

Para valores grandes de $\left|X_{t}\right|$ temos que $a_{1} \sim \phi_{1}$ e $a_{2} \sim \phi_{2}$ e para valores pequenos de $\left|X_{t}\right|$ temos que $a_{1} \sim \phi_{1}+\pi_{1}$ e $a_{2} \sim \phi_{2}+\pi_{2}$.

O modelo auto-regressivo exponencial de ordem 2 têm um comportamento semelhante ao modelo auto-regressivo com limiar mas os coeficientes mudam suavimente entre os dois extremos. 
Segundo Priestley (1988) o modelo auto-regressivo exponencial possui as seguintes características:

- dependência amplitude-freqüência;

- fenômeno de salto;

- ciclos limites.

\subsubsection{Modelo auto-regressivo com coeficientes aleatórios}

Andel (1976) verificou que quando modelamos séries hidrológicas, metereológicas e biológicas os coeficientes do modelo considerado, resultam de um complicado processo que usualmente possui características aleatórias. Estas características fizeram com que Andel considerasse modelos auto-regressivos com coeficientes aleatórios.

Definição 4.4: Uma série temporal $X_{t}\{t=0, \pm 1, \pm 2, \ldots\}$ segue um modelo autoregressivo com coeficientes aleatórios de ordem $\mathrm{k}, \mathrm{RCA}(\mathrm{k})$, se satisfizer a equação

$$
X_{t}=\sum_{i=1}^{k}\left[\beta_{i}+B_{i}(t)\right] X_{t-i}+e_{t}
$$

em que

$e_{t}$ são variáveis aleatórias independentes e identicamente distribuídas com média zero e variância $\sigma^{2}$;

$\beta_{\mathrm{i}}, \mathrm{i}=1, \ldots, \mathrm{k}$ são constantes;

$B(t)=\left[B_{1}(t), \ldots, B_{k}(t)\right]$ é uma seqüência de vetores aleatórios independentes com média zero e $\mathrm{E}\left[\mathrm{B}^{\mathrm{t}}(\mathrm{t}) \mathrm{B}(\mathrm{t})\right]=\mathrm{C}$;

$B(t)$ é independente de $e_{t}$. 
Notamos através de (4.17) que se os elementos de $\mathrm{C}$ forem pequenos comparados com os valores de $\beta_{i}$ o modelo é semelhante ao modelo autoregressivo dado por (3.9). Quando os valores de $\mathrm{B}_{\mathrm{i}}(\mathrm{t})$ forem grandes comparados com os valores de $\beta_{\mathrm{i}}$ a série $\mathrm{X}_{\mathrm{t}}$ terá alguns valores grandes especialmente se vários elementos de $\mathrm{C}$ forem relativamente grandes. Este é um comportamento que geralmente está associado com a não estacionariedade, mas pode ser uma indicação de não linearidade do modelo RCA.

Nas Figuras 4.14 e 4.15 apresentamos 1000 observações geradas pelos modelos RCA(1)

(vi) $\mathrm{X}_{\mathrm{t}}=\left(0,1+\mathrm{B}_{1}(\mathrm{t})\right) \mathrm{X}_{\mathrm{t}-1}+\mathrm{e}_{\mathrm{t}}$

(vii) $X_{t}=\left(0,5+B_{1}(t)\right) X_{t-1}+e_{t}$

com $e_{t} \sim N(0,1)$ independentes, $B_{1}(t) \sim N(0,1)$ independentes e $e_{t}$ independente de $\mathrm{B}_{1}(\mathrm{t})$.

Notamos pelas Figuras 4.14 e 4.15 que a série dada pelo modelo (4.18) apresenta um número de explosões de grande amplitude e que a série dada pelo modelo (4.19) apresenta uma forma extrema deste efeito.

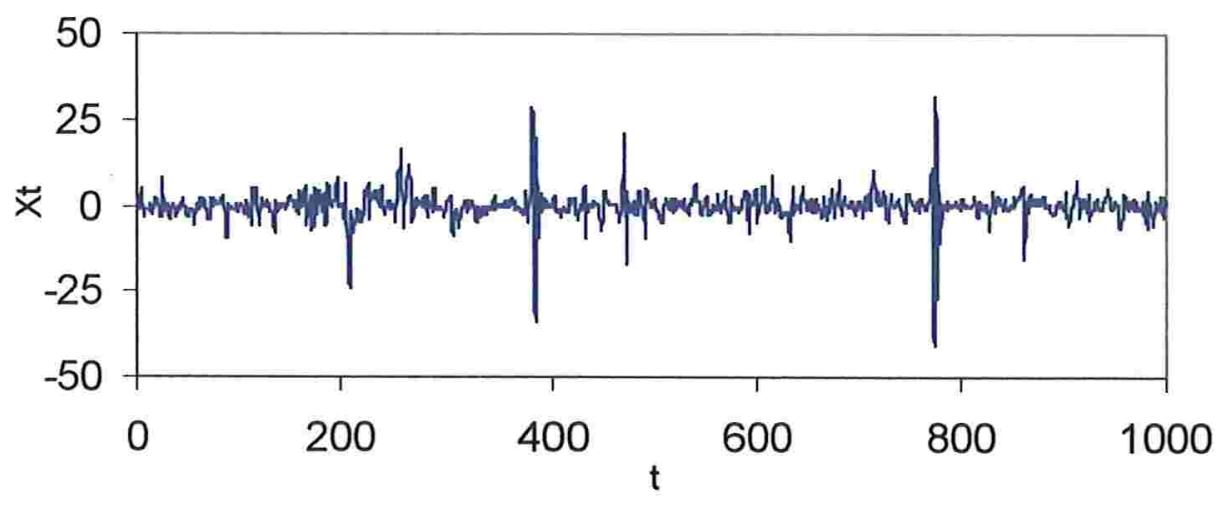

Figura 4.14 - Série gerada pelo modelo (4.18). 


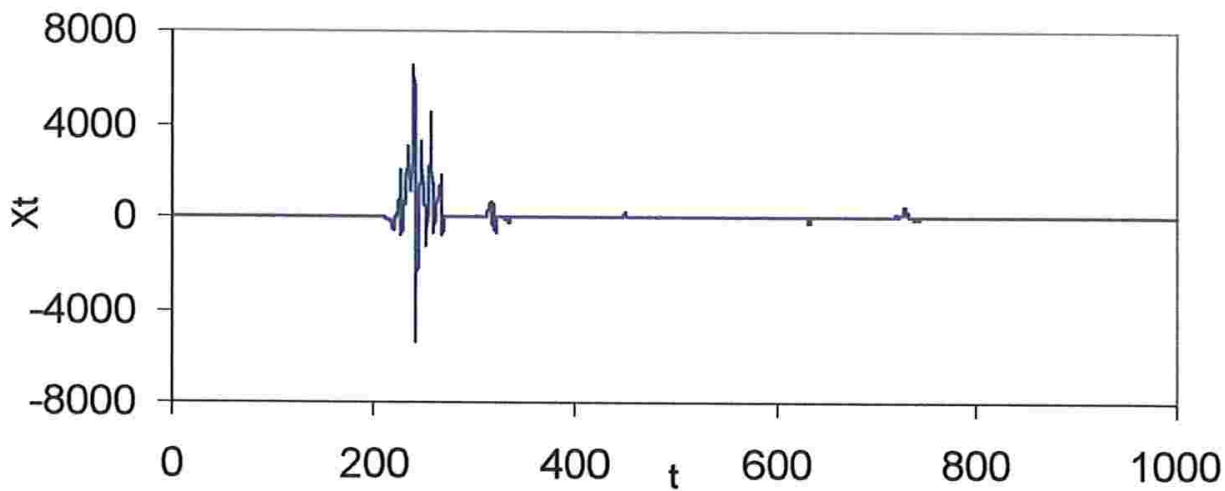

Figura 4.15 - Série gerada pelo modelo (4.19).

As condições de estacionariedade do modelo RCA podem ser encontradas em Nicholls e Quinn (1982).

\subsubsection{Modelo auto-regressivo com heterocedasticidade condicional - ARCH}

Os modelos ARCH foram introduzidos por Engle (1982). Esses modelos foram criados com o objetivo de modelar de uma forma conveniente a variância condicional de uma série temporal. Os modelos para séries temporais convencionais e os modelos econométricos trabalham com a suposição de que a série temporal possui variância constante. Os modelos $\mathrm{ARCH}$ permitem que a variância condicional possa mudar com o tempo, como uma função dos erros passados, deixando uma variância não condicional constante. Estes modelos são muito utilizados em diferentes fenômenos econômicos.

Definição 4.5: Uma série temporal $X_{t}\{t=0, \pm 1, \pm 2, \ldots\}$ segue um modelo autoregressivo com heterocedasticidade condicional, $\mathrm{ARCH}(\mathrm{p})$, se satisfizer as equações 


$$
\begin{gathered}
\mathrm{X}_{\mathrm{t}}=\mathrm{z}_{\mathrm{t}}^{\prime} \beta+\mathrm{e}_{\mathrm{t}}, \\
\mathrm{e}_{\mathrm{t}}=\varepsilon_{\mathrm{t}} \sigma_{\mathrm{t}},
\end{gathered}
$$

e

$$
\sigma^{2}{ }_{\mathrm{t}}=\alpha_{0}+\alpha_{1} \mathrm{e}^{2}{ }_{\mathrm{t}-1}+\ldots+\alpha_{\mathrm{p}} \mathrm{e}^{2}{ }_{\mathrm{t}-\mathrm{p}},
$$

em que

$\alpha_{0}>0$ e $\alpha_{i} \geq 0 i=1, \ldots, p ;$

$\sigma_{t}^{2}$ é variância do processo no instante t condicional a $\left(X_{1}, \ldots, X_{t-1}\right)$;

$\varepsilon_{\mathrm{t}}$ são variáveis aleatórias independentes e identicamente distribuídas com distribuição normal padrão;

$z_{1}$ é um processo determinístico ou estocástico que representa a média condicional de $X_{t}$;

$\beta$ é um parâmetro desconhecido.

\section{Algumas propriedades do modelo ARCH}

Exploraremos algumas propriedades do modelo $\mathrm{ARCH}(1) \operatorname{com} \beta=0$ dado por

$$
X_{t}=\varepsilon_{t} \sigma_{t}
$$

e

$$
\sigma^{2}{ }_{t}=\alpha_{0}+\alpha_{1} e^{2}{ }_{t-1}
$$

A série $X_{t}$ dado seus valores passados $\left(X_{1}, \ldots, X_{t-1}\right)$, que denotaremos por $\mathrm{X}_{\mathrm{t}} /\left(\mathrm{X}_{1}, \ldots, \mathrm{X}_{\mathrm{t}-1}\right)$, possui distribuição normal com média zero e variância $\sigma_{\mathrm{t}}^{2}$.

Os momentos não condicionais de $\mathrm{X}_{\mathbf{t}}$ são dados por: 
i) $\mathrm{E}\left(\mathrm{X}_{\mathrm{t}}\right)=\mathrm{E}\left(\sigma_{\mathrm{t}} \varepsilon_{\mathrm{t}}\right)=\mathrm{E}\left(\sigma_{\mathrm{t}}\right) \mathrm{E}\left(\varepsilon_{\mathrm{t}}\right)=0$ se $\mathrm{E}\left(\left|\sigma_{\mathrm{t}}\right|\right)<\infty$;

ii) $\mathrm{E}\left(\mathrm{X}_{\mathrm{t}}^{2}\right)=\frac{\alpha_{0}}{1-\alpha_{1}}$ para $\alpha_{1}<1$;

iii) $\mathrm{E}\left(\mathrm{X}_{\mathrm{t}}^{3}\right)=\mathrm{E}\left(\sigma^{3}{ }_{\mathrm{t}} \varepsilon_{\mathrm{t}}{ }_{\mathrm{t}}\right)=\mathrm{E}\left(\sigma^{3}{ }_{\mathrm{t}}\right) \mathrm{E}\left(\varepsilon_{\mathrm{t}}{ }_{\mathrm{t}}\right)=0$

iv)Todos os outros momentos de ordem ímpar, dado que o momento de ordem par superior seguinte exista, são iguais a zero;

v) $\mathrm{E}\left(\mathrm{X}_{\mathrm{t}}^{4}\right)=\mathrm{E}\left(\sigma_{\mathrm{t}}^{4}\right) \mathrm{E}\left(\varepsilon_{\mathrm{t}}^{4}\right)=3 \mathrm{E}\left(\sigma_{\mathrm{t}}^{4}\right)=3 \frac{\alpha_{0}^{2}\left(1+\alpha_{1}\right)}{\left(1-\alpha_{1}\right)\left(1-3 \alpha^{2}{ }_{1}\right)}$, para $3 \alpha^{2}{ }_{1}<1$.

O r-ésimo momento de um processo $\operatorname{ARCH}(1) \operatorname{com} \alpha_{0}>0$ e $\alpha_{1} \geq 0$ existe se e somente se $\alpha_{1}^{\mathrm{r}} \prod_{\mathrm{j}=1}^{\mathrm{r}}(2 \mathrm{j}-1)<1$.

Com base nos momentos dados acima podemos concluir que:

i) $\mathrm{X}_{\mathrm{t}}$ é um processo ruído branco se $0 \leq \alpha_{1}<1$;

ii) $\mathrm{X}_{\mathrm{t}}^{2}$ segue um processo auto-regressivo;

iii) $\mathrm{X}_{\mathrm{t}}^{2}$ é fracamente estacionário se $3 \alpha^{2}{ }_{1}<1$;

iv)Quando $X_{t}^{2}$ for um processo fracamente estacionário sua função de autocorrelação é dada por $\rho_{\mathrm{X}^{2}{ }_{\mathrm{t}}}(\mathrm{s})=\alpha_{1}^{\mathrm{s}}$; 
v)A medida de curtose de $X_{t}$, quando $3 \alpha_{1}^{2}<1$ é dada por $\mathrm{c}=\frac{\mathrm{E}\left(\mathrm{X}_{\mathrm{t}}^{4}\right)}{\left(\mathrm{E}\left(\mathrm{X}_{\mathrm{t}}^{2}\right)\right)^{2}}=3 \frac{1-\alpha_{1}^{2}}{1-3 \alpha_{1}^{2}}$. Devemos notar que quando $\alpha_{1} \neq 0$ a medida de curtose é maior que 3 , ou seja, $X_{t}$ possui leptocurtose (caudas pesadas).

vi) $X_{t}$ é uma diferença martingala se $E\left(\left|\sigma_{t}\right|\right)<\infty$.

Para obtermos as condições de estacionariedade de um modelo $\mathrm{ARCH}(\mathrm{p})$ vamos considerar a equação

$$
\sigma^{2}{ }_{t}=\alpha_{0}+\sum_{i=1}^{p} \alpha_{i} e_{t-i}^{2}
$$

que pode ser escrita como

$$
\sigma^{2}=\alpha_{0}+\alpha(B) e_{t}^{2}
$$

em que $\alpha(B)=\sum_{i=1}^{p} \alpha_{i} B^{i}$

Um processo $\mathrm{ARCH}(\mathrm{p})$ com $\alpha_{0}>0$ e $\alpha_{1}, \ldots, \alpha_{\mathrm{p}} \geq 0$ é fracamente estacionário se e somente se as raizes da equação 1- $\alpha(\mathrm{B})$ estiverem fora do círculo unitário. A variância estacionária é dada por $E\left(X_{t}^{2}\right)=\frac{\alpha_{0}}{1-\sum_{j=1}^{p} \alpha_{j}}$.

A função de autocorrelação de $\mathrm{X}_{\mathrm{t}}^{2}$, quando o processo é estacionário, não reflete a baixa autocorrelação de primeira ordem e posterior queda muito lenta para zero encontradas empiricamente. Se $\alpha_{1}$ for pequeno teremos baixa autocorrelação 
de primeira ordem, mas o decaimento será muito rápido. Se $\alpha_{1}$ for próximo de $1 \mathrm{o}$ decaimento será mais lento, mas a autocorrelação de primeira ordem será alta, contradizendo os resultados empíricos. Devido a esta inadequação houve a necessidade da busca de outros modelos que capturassem melhor as características empíricas das funções de autocorrelação de $\mathrm{X}_{\mathrm{t}}^{2}$.

\subsubsection{Modelo auto-regressivo generalizado com heterocedasticidade condicional - GARCH}

Os modelos GARCH foram introduzidos por Bollerslev (1986). Essa classe de modelos surgiu como uma generalização dos modelos $\mathrm{ARCH}$ descritos na sessão 4.3.5. Estes modelos permitem uma estrutura de defasagem mais flexível que a estrutura de defasagem dos modelos $\mathrm{ARCH}$. A extensão do processo $\mathrm{ARCH}$ para o GARCH tem muita semelhança com a extensão do processo AR para o processo ARMA.

Definição 4.6: Uma série temporal $\mathrm{X}_{\mathrm{t}}\{\mathrm{t}=0, \pm 1, \pm 2, \ldots\}$ segue um modelo autoregressivo generalizado com heterocedasticidade condicional, $\operatorname{GARCH}(\mathrm{p}, \mathrm{q})$, se satisfizer as equações

$$
\begin{gathered}
X_{t}=z_{t}^{\prime} \beta+e_{t}, \\
e_{t}=\varepsilon_{t} \sigma_{t},
\end{gathered}
$$

$\mathrm{e}$

$$
\sigma^{2}=\alpha_{0}+\sum_{i=1}^{p} \alpha_{i} X_{t-i}^{2}+\sum_{i=1}^{q} \gamma_{i} \sigma_{t-1}^{2}
$$

em que

$p>0, q \geq 0, \alpha_{0}>0, \alpha_{i} \geq 0 i=1, \ldots, p$ e $\gamma_{j} \geq 0, j=1, \ldots, q ;$ 
$\varepsilon_{\mathrm{t}}$ são variáveis aleatórias independentes e identicamente distribuídas com distribuição normal padrão;

$z_{\mathrm{t}}$ é um processo determinístico ou estocástico que representa a média condicional de $X_{t}$;

$\beta$ é um parâmetro desconhecido.

Quando $\mathrm{q}=0$ o processo se reduz a um modelo $\mathrm{ARCH}(\mathrm{p})$. Nos modelos $\mathrm{ARCH}(\mathrm{p})$ a variância condicional é especificada como uma função linear somente das variâncias passadas, enquanto que nos modelos GARCH ela é especificada também como função linear das observações passadas da série temporal.

\section{Propriedades do modelo GARCH}

Exploraremos algumas propriedades do modelo GARCH mais simples e mais utilizado que é o modelo $\operatorname{GARCH}(1,1) \operatorname{com} \beta=0$ que é dado por

$$
\begin{gathered}
\mathrm{X}_{\mathrm{t}}=\varepsilon_{\mathrm{t}} \sigma_{\mathrm{t}}=\mathrm{e}_{\mathrm{t}} \\
\sigma^{2}{ }_{\mathrm{t}}=\alpha_{0}+\alpha_{1} \mathrm{X}_{\mathrm{t}-1}^{2}+\gamma_{1} \sigma_{\mathrm{t}-1}^{2}
\end{gathered}
$$

ou

$$
\sigma^{2}{ }_{t}=\alpha_{0}+\alpha_{1} e_{t-1}^{2}+\gamma_{1} \sigma_{t-1}^{2}
$$

em que $\alpha_{0}>0, \quad \alpha_{1}>0$ e $\gamma_{1}>0$ e $\varepsilon_{\mathrm{t}}$ são variáveis aleatórias independentes e identicamente distribuídas com distribuição normal padrão;

Em termos dos quadrados de $X_{t}$, a expressão (4.30) pode ser escritas como

$$
X^{2}{ }_{t}=\alpha_{0}+\left(\alpha_{1}+\gamma_{1}\right) X_{t-1}^{2}-\gamma_{1} v_{t-1}+v_{t},
$$

em que $v_{t}=\sigma_{t}^{2}\left(\varepsilon_{t}^{2}-1\right)$. 
Notamos pela expressão (4.33) que o processo $\operatorname{GARCH}(1,1)$ pode ser interpretado como um processo $\operatorname{ARMA}(1,1) \mathrm{em} \mathrm{X}^{2}$.

Para $\alpha_{1} \neq 0$ a função de autocorrelação do processo $\mathrm{X}^{2}{ }_{\mathrm{t}}$ é dada por

$$
\rho_{1}=\frac{\left(1-\gamma_{1}\left(\alpha_{1}+\gamma_{1}\right)\right) \alpha_{1}}{1+\gamma_{1}^{2}-2 \gamma_{1}\left(\alpha_{1}+\gamma_{1}\right)}
$$

e

$$
\rho_{\mathrm{s}}=\left(\alpha_{1}+\gamma_{1}\right)^{\mathrm{s}-1} \rho_{1} \text { para } \mathrm{s}>1
$$

Vimos que para o processo $\mathrm{ARCH}(1)$ a função de autocorrelação de $\mathrm{X}^{2}{ }_{1}$ quando o processo é estacionário não reflete a baixa autocorrelação de primeira ordem e posterior queda muito lenta para zero encontradas empiricamente. A função de autocorrelação do processo $\operatorname{GARCH}(1,1)$ é capaz de suprir tal deficiência desde que $\alpha_{1}$ seja pequeno e $\alpha_{1}+\gamma_{1}$ grande.

Segundo Bollerslev (1986) a condição necessária e suficiente para que o momento de ordem $2 \mathrm{r}$ exista é que

$$
\phi\left(\alpha_{1}, \gamma_{1}, r\right)=\sum_{j=0}^{r}\left(\begin{array}{l}
r \\
j
\end{array}\right) \alpha_{j} \alpha^{j}{ }_{1} \gamma_{1}^{r-j}<1
$$

em que $\alpha_{0}=1, \alpha_{j}=\prod_{i=1}^{j}(2 i-1) j=1,2, \ldots, r$.

Os momentos de ordem (2r-1) são iguais a zero e os momentos de ordem 2r são dados por

$$
E\left(X_{t}^{2 r}\right)=\alpha_{r}\left(\sum_{k=0}^{r-1} \alpha_{k}^{-1} E\left(X_{t}^{2 k}\right) \alpha_{0}^{r-k}\left(\begin{array}{l}
r \\
r-k
\end{array}\right) \phi\left(\alpha_{1}, \gamma_{1}, k\right)\right)\left(1-\phi\left(\alpha_{1}, \gamma_{1}, r\right)\right)^{-1}
$$


Um processo $\operatorname{GARCH}(\mathrm{p}, \mathrm{q})$ é estacionário se os coeficientes $\alpha_{i}$ e $\gamma_{\mathrm{i}}$ forem não negativos e $\alpha(1)+\gamma(1)<1$.

Há um número muito grande de extensões dos modelos $\mathrm{ARCH}$ (ver Bollerslev, Chou e Kroner (1992)) como, por exemplo, o GARCH assimétrico (AGARCH), o GARCH exponencial (EGARCH), o GARCH integrado (IGARCH) e o ARCH em média (ARCH-M), que não abordaremos neste trabalho. 


\section{Capítulo 5}

\section{Testes de linearidade e normalidade no domínio de freqüências}

Um problema prático encontrado ao ajustar um modelo a uma série temporal é decidir se devemos utilizar um modelo não-linear. Os testes de linearidade podem ser utilizados para auxiliar nesta decisão.

Os testes de linearidade podem ser classificados em três tipos principais: paramétricos ou semi-paramétricos, não paramétricos baseados no domínio de freqüências e não paramétricos baseados no domínio do tempo.

Abordaremos, neste capítulo os testes não paramétricos baseados no domínio de freqüências, que foram originalmente desenvolvidos por Subba Rao e Gabr (1980) e, posteriormente, por Hinich (1982), Ashley et al. (1986), Brockett et al. (1988), Priestley (1988) e Brilliger e Irizarry (1998). O desenvolvimento desses testes baseia-se no fato de que quando um processo possui distribuição normal, todos os poliespectros de ordem maior do que dois são iguais a zero. Desta forma quando um processo possui um bi-espectro diferente de zero, ou seja $f_{x}\left(\omega_{1}, \omega_{2}\right) \neq 0$, temos uma das duas situações seguintes: 
(1) O processo satisfaz um modelo linear da forma $\sum_{u=0}^{\infty} g_{u} e_{t-u}$ mas $\{e\}$ não tem distribuição normal

ou

(2) O processo satisfaz um modelo não-linear da forma

$$
X_{t}=\mu+\sum_{u=0}^{\infty} g_{u} e_{t-u}+\sum_{u=0}^{\infty} \sum_{v=0}^{\infty} g_{u v} e_{t-u} e_{t-v}+\sum_{u=0}^{\infty} \sum_{v=0}^{\infty} \sum_{w=0}^{\infty} g_{u w w} e_{t-u} e_{t-v} e_{t-w}+\ldots
$$

em que $\left\{e_{t}\right\}$ pode ou não ter distribuição normal.

Assim, a análise bi-espectral é muito importante para detectar a normalidade e a linearidade de uma série temporal.

Os testes de normalidade e linearidade para o bi-espectro podem ser aplicados à série original ou ao resíduo ajustado de um modelo linear devido ao seguinte resultado de Ashley, Hinich and Patterson (1986):

Seja $\left\{X_{t}\right\}$ um processo estocástico discreto com média zero e estacionário de terceira ordem possuindo uma função de autocovariância de terceira ordem absolutamente somável. Se $\left\{Y_{t}\right\}$ é gerado por um processo $\left\{X_{t}\right\}$ por meio de um filtro linear com função resposta de impulso absolutamente somável, então $\left\{X_{t}\right\}$ e $\left\{Y_{t}\right\}$ possuem idênticas funções de assimetria quadrática.

\subsection{Testes de Subba Rao e Gabr}

Subba Rao e Gabr (1980) inspirados em Brillinger (1965) desenvolveram testes com o objetivo de testar as seguintes hipóteses: 
- $\mathrm{H}_{01}$ :o processo é gaussiano;

- $\mathrm{H}_{02}$ : o processo se comporta como um modelo linear.

Inicialmente deve ser testada a hipótese $\mathrm{H}_{01}$; caso esta hipótese não seja rejeitada, isto é, se o processo for gaussiano e, além disso, o processo for estacionário, com espectro absolutamente contínuo, ele poderá ser representado por um modelo linear e a hipótese $\mathrm{H}_{02}$ não precisa ser testada. Quando a hipótese $\mathrm{H}_{01}$ for rejeitada, ou seja quando o processo não for gaussiano, é necessário testar a hipótese $\mathrm{H}_{02}$.

Para testar se o processo é gaussiano, devemos testar a hipótese de que o bi-espectro é zero para todo conjunto de freqüências $\left(\omega_{1}, \omega_{2}\right)$, isto é,

$$
\mathrm{H}_{01}: \mathrm{f}_{\mathrm{N}}\left(\omega_{1}, \omega_{2}\right) \equiv 0, \quad \forall\left(\omega_{1}, \omega_{2}\right)
$$

A estatística deste teste é construída utilizando estimativas do bi-espectro sobre uma grade adequada de freqüências.

Sob a hipótese de que o modelo é linear temos que

$$
X_{t}=\sum_{u=-\infty}^{+\infty} g_{u} e_{t-u},
$$

$\operatorname{com} \sum_{u=-\infty}^{+\infty} g^{2} u<\infty$.

Como e é uma seqüência de variáveis aleatórias independentes e identicamente distribuídas com $\mathrm{E}\left(\mathrm{e}_{\mathrm{t}}\right)=0, \mathrm{E}\left(\mathrm{e}^{2}\right)=\sigma_{\mathrm{e}}^{2} \mathrm{e} \mathrm{E}\left(\mathrm{e}^{3}\right)=\beta$, temos que 


$$
f_{x}(\omega)=\frac{\sigma_{e}^{2}}{2 \pi}|H(\omega)|^{2}
$$

e

$$
f_{x}\left(\omega_{1}, \omega_{2}\right)=\frac{\beta}{(2 \pi)^{2}} H\left(-\omega_{1}-\omega_{2}\right) H\left(\omega_{1}\right) H\left(\omega_{2}\right),
$$

em que $H(\omega)=\sum_{-\infty}^{+\infty} g_{u} e^{-i \omega u}$

Das expressões (5.3) e (5.4) temos que a bi-coerência é dada por

$$
\gamma^{2}\left(\omega_{1}, \omega_{2}\right)=\frac{\left|f_{x}\left(\omega_{1}, \omega_{2}\right)\right|^{2}}{f_{x}\left(\omega_{1}\right) f_{x}\left(\omega_{2}\right) f_{x}\left(\omega_{1}+\omega_{2}\right)}=\frac{\beta^{2}}{2 \pi \sigma_{e}^{2}}
$$

e é constante para todo $\left(\omega_{1}, \omega_{2}\right)$.

A expressão (5.5) mostra que se $\beta=0$ então a função densidade bi-espectral $\mathrm{f}_{\mathrm{x}}\left(\omega_{1}, \omega_{2}\right)=0$, para todo par $\left(\omega_{1}, \omega_{2}\right)$. Quando as variáveis aleatórias e possuem distribuição normal então $\beta=0$ e $\mathrm{f}_{\mathrm{x}}\left(\omega_{1}, \omega_{2}\right)=0$. Sob condições adequadas da seqüência $g_{u}$ pode ser mostrado que a normalidade de $e_{1}$ implica na normalidade $X_{t}$. Então podemos concluir que se $X_{t}$ é um processo gaussiano então $f_{X}\left(\omega_{1}, \omega_{2}\right)=0$ para todo par $\left(\omega_{1}, \omega_{2}\right)$. Entretanto, o processo $X_{t}$ pode ser linear mas não necessariamente gaussiano. Subba Rao e Gabr observaram que nesta situação a bicoerência dada pela expressão (5.5) é constante para todo $\left(\omega_{1}, \omega_{2}\right)$. Desta forma a constância da bi-coerência é um teste para a adequação da linearidade do processo (Brillinger, 1965).

Logo, para testar se o processo se comporta como um modelo linear, devemos testar a hipótese de que a bi-coerência é constante para todo conjunto de freqüências $\left(\omega_{1}, \omega_{2}\right)$, isto é 


$$
\mathrm{H}_{02}: \gamma^{2}\left(\omega_{1}, \omega_{2}\right)=\text { constante, } \forall\left(\omega_{1}, \omega_{2}\right) \text {. }
$$

$O$ teste para linearidade é baseado na substituição de $f_{x}\left(\omega_{1}, \omega_{2}\right)$ e $f_{x}(\omega)$ por seus respectivos estimadores.

Veremos a seguir, com detalhes, a construção dos testes citados anteriormente.

\subsubsection{Teste de normalidade}

O objetivo deste teste é testar a hipótese

$$
\mathrm{H}_{01}: \mathrm{f}_{\mathrm{x}}\left(\omega_{\mathrm{i}}, \omega_{\mathrm{j}}\right) \equiv 0, \forall\left(\omega_{\mathrm{i}}, \omega_{\mathrm{j}}\right)
$$

O teste proposto é feito em dois estágios:

\section{i) Estágio 1}

Neste estágio testamos a hipótese

$$
\mathrm{H}_{01}: \mathrm{f}_{\mathrm{x}}\left(\omega_{\mathrm{i}}, \omega_{\mathrm{j}}\right) \equiv 0 \text {, para } 0<\omega_{\mathrm{i}}<\frac{2 \pi}{3} \text { e } \omega_{\mathrm{i}}<\omega_{\mathrm{j}}<\pi-\frac{\omega_{\mathrm{i}}}{2}
$$

Nesta região o bi-espectro estimado possui distribuição normal complexa e a estatística utilizada é a análoga complexa da estatística $\mathrm{T}^{2}$ de Hotelling, ver Giri (1965) e Khatri (1965).

Para testar $\mathrm{H}_{01}$ dividiremos o intervalo $[0, \pi]$ em $\mathrm{K}$ subintervalos e selecionaremos um conjunto de freqüências $\left(\omega_{\mathrm{i}}, \omega_{\mathrm{j}}\right)$, em que 


$$
\omega_{i}=\frac{\pi \mathrm{i}}{\mathrm{K}}, \omega_{\mathrm{j}}=\frac{\pi \mathrm{j}}{\mathrm{K}} \text { para } \mathrm{i}=1,2, \ldots, \mathrm{L} \text { e } \mathrm{j}=\mathrm{i}+1, \mathrm{i}+2, \ldots, \mathrm{M}(\mathrm{i})
$$

com $\mathrm{L}=\left[\frac{2 \mathrm{~K}}{3}\right], \mathrm{M}(\mathrm{i})=\mathrm{K}-\left[\frac{\mathrm{i}}{2}\right]-1, \mathrm{~K}<<\mathrm{T}$ e $\mathrm{T}$ é o número de observações utilizadas no cálculo das estimativas bi-espectrais.

Nas colunas de (1) a (4) da Tabela 5.1 estão os pares de freqüências $\left(\omega_{i}, \omega_{j}\right)$ para $\mathrm{K}=4$ e 6 .

Tabela 5.1 - Pares de freqüências $\left(\omega_{\mathrm{i}}, \omega_{\mathrm{j}}\right)$, definidas por $(5.6)$

\begin{tabular}{|c|c|c|c|c|}
\hline \multicolumn{2}{|c|}{$\mathrm{K}=4$} & \multicolumn{2}{|c|}{$K=6$} & $\mathrm{~K}=6$ \\
\hline$\omega_{\mathrm{i}}$ & $\omega_{j}$ & $\omega_{i}$ & $\omega_{\mathrm{j}}$ & $\mathrm{f}_{\mathrm{x}}\left(\omega_{\mathrm{i}}, \omega_{\mathrm{j}}\right)$ \\
\hline$\pi / 4$ & $2 \pi / 4$ & $\pi / 6$ & $2 \pi / 6$ & $f_{X}\left(\frac{\pi}{6}, \frac{2 \pi}{6}\right)=\xi_{1}$ \\
\hline \multirow[t]{6}{*}{$\pi / 4$} & $3 \pi / 4$ & $\pi / 6$ & $3 \pi / 6$ & $\mathrm{f}_{\mathrm{x}}\left(\frac{\pi}{6}, \frac{3 \pi}{6}\right)=\xi_{2}$ \\
\hline & & $\pi / 6$ & $4 \pi / 6$ & $f_{X}\left(\frac{\pi}{6}, \frac{4 \pi}{6}\right)=\xi_{3}$ \\
\hline & & $\pi / 6$ & $5 \pi / 6$ & $\mathrm{f}_{\mathrm{X}}\left(\frac{\pi}{6}, \frac{5 \pi}{6}\right)=\xi_{4}$ \\
\hline & & $2 \pi / 6$ & $3 \pi / 6$ & $f_{X}\left(\frac{2 \pi}{6}, \frac{3 \pi}{6}\right)=\xi_{5}$ \\
\hline & & $2 \pi / 6$ & $4 \pi / 6$ & $f_{X}\left(\frac{2 \pi}{6}, \frac{4 \pi}{6}\right)=\xi_{6}$ \\
\hline & & $3 \pi / 6$ & $4 \pi / 6$ & $f_{X}\left(\frac{3 \pi}{6}, \frac{4 \pi}{6}\right)=\xi_{7}$ \\
\hline
\end{tabular}

Seja $\xi_{\mathrm{i}}$ o bi-espectro calculado no i-ésimo par de freqüências dado por (5.6) e ๆ'o vetor formado por estes bi-espectros, isto é,

$$
\eta^{\prime}=\left(\xi_{1}, \xi_{2}, \ldots, \xi_{\mathrm{P}}\right)
$$


em que $P=\sum_{i=1}^{L}(M(i)-i)$

Para obtermos um conjunto de estimativas aproximadamente não correlacionadas para cada $\xi_{\mathrm{i}}$ precisamos construir uma grade de freqüências em torno de cada ponto $\left(\omega_{\mathrm{i}}, \omega_{\mathrm{j}}\right)$. Desta forma para cada $\left(\omega_{\mathrm{i}}, \omega_{\mathrm{j}}\right)$ temos

$$
\omega_{\text {ip }}=\omega_{i}+\frac{p d \pi}{T} \quad p=-r,-(r-1), \ldots, 0,1, \ldots, r
$$

e

$$
\omega_{j q}=\omega_{j}+\frac{q d \pi}{T} \quad q=-r,-(r-1), \ldots,-1,1, \ldots, r(q \neq 0),
$$

em que a distância d é escolhida tal que as estimativas bi-espectrais nos pontos vizinhos desta grade são aproximadamente não correlacionadas. Desta forma, $\frac{d \pi}{T}$ deve ser maior que a largura de faixa da janela espectral, correspondente à janela $\lambda\left(\mathrm{s}_{1}, \mathrm{~s}_{2}\right)$ utilizada na estimação do bi-espectro.

Na Figura 5.1 está representada a grade de freqüências em torno de cada par de freqüências da Tabela 5.1, para $K=6$ e $r=2$.

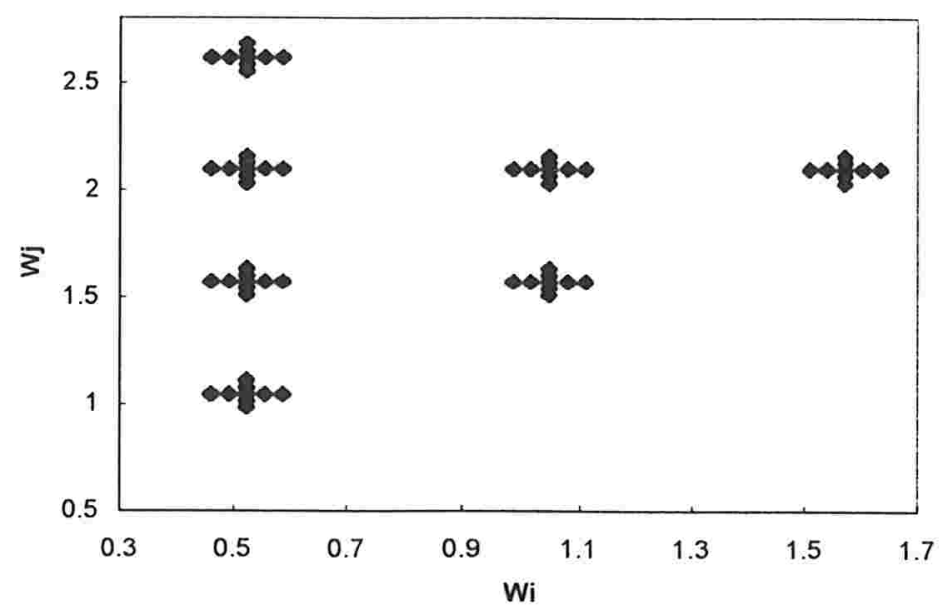

Figura 5.1 - Grade de freqüências em torno das freqüências $\left(\omega_{\mathrm{i}}, \omega_{\mathrm{j}}\right)$ das colunas (3) e (4) da Tabela 5.1. 
A função densidade bi-espectral nas freqüências $\left(\omega_{\mathrm{i}}, \omega_{\mathrm{j}}\right)$ é estimada por meio de (2.20) e de um gerador de "lag window" da Tabela 2.1.

Assumindo que a verdadeira função densidade bi-espectral é suficientemente suavizada e aproximadamente constante na grade de freqüências dada por (5.8) e (5.9), temos que

$$
\mathrm{E}\left[\hat{\mathrm{f}}\left(\omega_{\mathrm{ip}}, \omega_{\mathrm{jq}}\right)\right] \approx \mathrm{f}_{\mathrm{x}}\left(\omega_{\mathrm{i}}, \omega_{\mathrm{j}}\right), \quad \forall \mathrm{p}, \mathrm{q} .
$$

Vamos considerar o conjunto, $\left\{\hat{f}\left(\omega_{\text {ip }}, \omega_{i q}\right)\right\}$, de $n_{1}=4 r+1$ estimadores aproximadamente não correlacionados e não viciados de $f_{x}\left(\omega_{i}, \omega_{j}\right)$ dado por

$$
\begin{gathered}
\left\{\hat{f}\left(\omega_{i}-\frac{r d \pi}{T}, \omega_{j}\right), \hat{f}\left(\omega_{i}-\frac{(r-1) d \pi}{T}, \omega_{j}\right), \ldots, \hat{f}\left(\omega_{i}, \omega_{j}\right), \ldots \hat{f}\left(\omega_{i}+\frac{r d \pi}{T}, \omega_{j}\right),\right. \\
\left.\hat{f}\left(\omega_{i}, \omega_{j}-\frac{r d \pi}{T}\right), \hat{f}\left(\omega_{i}, \omega_{j}-\frac{(r-1) d \pi}{T}\right), \ldots, \hat{f}\left(\omega_{i}, \omega_{j}+\frac{r d \pi}{T}\right)\right\} .
\end{gathered}
$$

Para cada elemento do vetor $\eta^{\prime}$ dado pela expressão (5.7) é necessário obter um vetor de $n_{1}$ estimadores dado pela expressão (5.11). O conjunto de todos os estimadores da função densidade bi-espectral é representado na matriz $\hat{D}$ dada por

$$
\hat{\mathrm{D}}=\left[\begin{array}{cccc}
\hat{\zeta}_{11} & \hat{\zeta}_{12} & \ldots & \hat{\zeta}_{1_{n_{1}}} \\
\hat{\zeta}_{21} & \hat{\zeta}_{22} & \ldots & \hat{\zeta}_{2 \mathrm{n}_{1}} \\
\vdots & \vdots & \vdots & \vdots \\
\hat{\zeta}_{\mathrm{P} 1} & \hat{\zeta}_{\mathrm{P} 2} & \ldots & \hat{\zeta}_{\mathrm{Pn}_{1}}
\end{array}\right]=\left[\hat{\zeta}_{1}, \ldots, \hat{\zeta}_{\mathrm{n}_{1}}\right]
$$


Para valores grandes de $\mathrm{T}$, cada $\hat{\zeta}_{\mathrm{i}}$ possui distribuição normal complexa com média $\eta$ e matriz de covariância $\Sigma_{\zeta}$. Os estimadores de máxima verossimilhança de $\eta$ e $\sum_{\zeta}$ são dados por

$$
\hat{\eta}=\frac{1}{n_{1}} \sum_{k=1}^{n_{1}} \zeta_{k} \text { e } \hat{\Sigma}_{\zeta}=\mathbf{A} / n_{1}
$$

em que $A=\sum_{k=1}^{n_{1}}\left(\zeta_{k}-\hat{\eta}\right)\left(\zeta_{k}-\hat{\eta}\right)^{*}$ com o símbolo * denotando o transposto do complexo conjugado.

O teste da razão de verossimilhança para testar

$$
\begin{aligned}
& \mathrm{H}_{0}: \eta=0 \\
& \mathrm{H}_{1}: \eta * \sum_{\zeta}^{-1} \eta>0
\end{aligned}
$$

leva à rejeição da hipótese nula se a estatística do teste

$$
\mathrm{T}^{2}=\mathrm{n}_{1} \hat{\eta}^{*} \mathbf{A}^{-1} \hat{\eta}
$$

for maior do que $\lambda$, em que $\lambda$ é determinado pelo nivel de significância $\alpha$.

Sob $\mathrm{H}_{0}$, a estatística

$$
\mathfrak{J}_{1}=\left[\frac{2\left(\mathrm{n}_{1}-\mathrm{P}\right)}{2 \mathrm{P}}\right] \mathrm{T}^{2},
$$


possui distribuição $\mathrm{F}(2 \mathrm{P}, 2(\mathrm{n} 1-\mathrm{P}))$. Se a hipótese nula não for rejeitada devemos ir para o segundo estágio do teste.

\section{ii) Estágio 2}

Neste estágio testamos a hipótese

$$
\mathrm{H}_{01}: \mathrm{f}_{\mathrm{x}}\left(\omega_{\mathrm{i}}, \omega_{\mathrm{j}}\right) \equiv 0
$$

para $\omega_{i}, \omega_{j}$ na vizinhança de $0<\omega_{i}<\frac{2 \pi}{3}$ e $\omega_{i}<\omega_{j}<\pi-\frac{\omega_{i}}{2}$ e na origem.

Neste caso a estatística do teste é a $\mathrm{T}^{2}$ de Hotelling para o caso real, ver Anderson (1958).

Para maior detalhes ver Subba Rao e Gabr (1980).

\subsubsection{Teste de linearidade}

$\mathrm{O}$ teste de linearidade descrito a seguir é baseado na propriedade de que se o modelo for linear a bi-coerência, dada por (5.5), é constante para todo $\left(\omega_{\mathrm{i}}, \omega_{\mathrm{j}}\right)$.

Seja $Y=\left(Y_{1}, Y_{2}, \ldots, Y_{P}\right)^{\prime}$ um vetor coluna de ordem Px1 em que para cada $1=1, \ldots, P, Y_{1}=\gamma^{2}\left(\omega_{i}, \omega_{j}\right)$, ou seja, $Y_{1}$ é a bi-coerência calculada em algum par de freqüências tal que $0<\omega_{i}<\frac{2 \pi}{3}$ e $\omega_{i}<\omega_{j}<\pi-\frac{\omega_{i}}{2}$. Neste teste utilizaremos os pares de freqüência $\left(\omega_{\mathrm{i}}, \omega_{\mathrm{j}}\right)$ das colunas (3) e (4) da Tabela 5.1 .

Para obtermos um conjunto de $n_{1}$ estimativas de cada $Y_{1}$ utilizaremos o estimador da bi-coerência dado pela expressão (2.24), calculado na grade de 
freqüências dada por (5.8) e (5.9). Estes estimadores possuem distribuição assintoticamente normal (ver Brillinger,1965). Desta forma obteremos uma amostra aleatória de $\mathrm{n}_{1}$ estimadores de $\mathbf{Y}$, que podem ser representados pela matriz $\hat{\mathbf{D}}_{1}$ dada por

$$
\hat{\mathbf{D}}_{1}=\left[\begin{array}{cccc}
\hat{\mathrm{Y}}_{11} & \hat{\mathrm{Y}}_{12} & \ldots & \hat{\mathrm{Y}}_{1 \mathrm{n}_{1}} \\
\hat{\mathrm{Y}}_{21} & \hat{\mathrm{Y}}_{22} & \ldots & \hat{\mathrm{Y}}_{2 \mathrm{n}_{1}} \\
\vdots & \vdots & \vdots & \vdots \\
\hat{\mathrm{Y}}_{\mathrm{P} 1} & \hat{\mathrm{Y}}_{\mathrm{P} 2} & \ldots & \hat{\mathrm{Y}}_{\mathrm{Pn}_{1}}
\end{array}\right]=\hat{\mathbf{Y}}_{1}, \ldots, \hat{\mathbf{Y}}_{\mathrm{n}_{1}} .
$$

em que $\hat{\mathrm{Y}}_{\mathrm{lm}}, \mathrm{l}=1, \ldots, \mathrm{P}$ e $\mathrm{m}=1, \ldots, \mathrm{n}_{1}$, é o m-ésimo estimador do l-ésimo elemento do vetor $\mathbf{Y}$.

Sob a hipótese,

\section{$\mathrm{H}_{02}$ : o processo é linear}

o vetor média de $\left\{\hat{\mathbf{Y}}_{\mathbf{i}}\right\}$ contém elementos idênticos. O teste de linearidade baseado em $\gamma^{2}\left(\omega_{i}, \omega_{j}\right)$ corresponde ao teste clássico de simetria em análise multivariada, ver Anderson (1958), descrito a seguir.

$$
\operatorname{Sejam} \overline{\mathbf{Y}}=\frac{1}{n_{1}} \sum_{i=1}^{n_{1}} \hat{\mathbf{Y}}_{i}, \hat{\mathbf{S}}_{\mathbf{y}}=\sum_{\mathbf{i}=1}^{\mathrm{n}_{1}}\left(\hat{\mathbf{Y}}_{\mathbf{i}}-\overline{\mathbf{Y}}\right)\left(\hat{\mathbf{Y}}_{\mathrm{i}}-\overline{\mathbf{Y}}\right)^{\prime} \text { e } \hat{\Sigma}_{\mathbf{y}}=\hat{\mathbf{S}}_{\mathbf{y}} / \mathrm{n}_{1}
$$

Vamos definir o vetor coluna $\beta^{1}$ de ordem $\mathrm{Qx} 1$, em que $\mathrm{Q}=\mathrm{P}-1$, tal que $\beta^{1}=\mathbf{B Y}$, em que $\mathbf{B}$ é uma matriz de ordem (QxP) da seguinte forma 


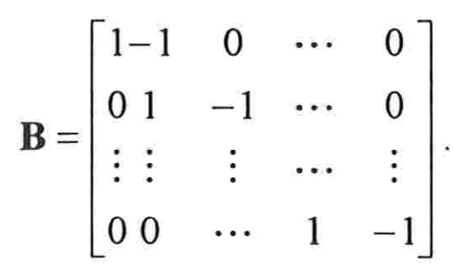

Sob $\mathrm{H}_{02}, \beta^{1}$ possui distribuição assintoticamente normal com vetor média zero e matriz de covariâncias dada por $\mathbf{B} \sum_{\mathbf{Y}} \mathbf{B}^{\prime}$. O teste da razão de verossimilhança leva à rejeição da hipótese se

$$
\mathrm{T}^{2}=\mathrm{n}_{1} \bar{\beta}^{1} \hat{\mathrm{S}}^{-1} \bar{\beta}^{1}>\lambda_{0}
$$

em que $\overline{\boldsymbol{\beta}}^{1}=\mathbf{B} \overline{\mathbf{Y}}, \hat{\mathbf{S}}=\mathbf{B} \hat{\mathbf{S}}_{\mathbf{Y}} \mathbf{B}^{\prime}$ e $\lambda_{0}$ é determinado pelo nivel de significância $\alpha$.

Sob $\mathrm{H}_{02}$, a estatística

$$
\Im_{2}=\left[\frac{\left(\mathrm{n}_{1}-\mathrm{Q}\right)}{\mathrm{Q}}\right] \mathrm{T}^{2}
$$

possui distribuição $F\left(Q, n_{1}-Q\right)$. A estatística $T^{2}$ é invariante e independe da escolha de B (Anderson, 1958)

\section{Escolha dos parâmetros}

Inicialmente devemos escolher $\mathrm{K}$ pontos igualmente espaçados no intervalo $(0, \pi)$ e formar uma grade de pontos, sendo que $\mathrm{K}$ deve ser menor que $\mathrm{T}$ (número de observações da série), para fornecer intervalos em torno de cada par de freqüências $\left(\omega_{\mathrm{i}}, \omega_{\mathrm{j}}\right)$ e desta forma permitir a construção de estimativas espectrais e bi-espectrais nos pontos vizinhos $\left(\omega_{\mathrm{ip}}, \omega_{\mathrm{jq}}\right)$ da grade. O número total de pontos em cada grade é $n_{1}=4 \mathrm{r}+1$ e visto que há $\mathrm{K}^{2} / 3$ grades, é necessário que 


$$
\frac{\mathrm{n}_{1} \mathrm{~K}^{2}}{3}<\mathrm{T}
$$

Para garantir que as estimativas espectrais e bi-espectrais nos diferentes pontos da grade sejam não correlacionadas é necessário que d seja escolhido tal que

$$
\frac{\pi \mathrm{d}}{\mathrm{T}}>\mathrm{J},
$$

em que $\mathrm{J}$ é a largura de faixa da janela espectral utilizada no procedimento de estimação.

Para que $f_{x}\left(\omega_{1}, \omega_{2}\right)$ e $f_{x}(\omega)$ sejam aproximadamente constantes sobre todos os pontos em cada grade é necessário que r seja escolhido tal que

$$
\frac{2 \pi \mathrm{r}}{\mathrm{T}}<\mathrm{J}
$$

em que $J$ é a largura de faixa da janela espectral utilizada no procedimento de estimação.

Para garantir que os pontos em diferentes grades não sobreponham-se é necessário que

$$
\frac{\mathrm{T}}{(\mathrm{K}(\mathrm{r}+1))}>\mathrm{d}
$$

Para implementar as restrições acima devemos ter informações a priori sobre a largura de faixa do espectro e do bi-espectro. Na prática esta informação raramente pode ser disponível e a escolha dos parâmetros é feita utilizando informações obtidas por meio da suavização do espectro e bi-espectro estimados. 
Na Tabela 5.2 estão alguns típicos valores de $\mathrm{K}, \mathrm{L}, \mathrm{P}, \mathrm{r}$ e $\mathrm{n}_{1}$ sugeridos por Subba Rao e Gabr (1980) para serem utilizados em aplicações.

Tabela 5.2 - Valores de (K,L,P,r, $\left.\mathrm{n}_{1}\right)$ sugeridos por Subba Rao e Gabr (1980)

\begin{tabular}{|c|c|c|c|c|}
\hline $\begin{array}{l}\mathrm{K}: \mathrm{n}^{0} \text { de subintervalos do } \\
\text { intervalo }[0, \pi]\end{array}$ & $\mathrm{L}=\left[\frac{2 \mathrm{~K}}{3}\right]$ & $\begin{array}{c}\mathrm{P}: \mathrm{n}^{0} \text { de pontos em que o } \\
\text { bi-espectro deve ser } \\
\text { calculado }\end{array}$ & $\mathrm{r}$ & $\mathrm{n}_{1}=4 \mathrm{r}+1$ \\
\hline 4 & 2 & 2 & $\geq 1$ & $\geq 5$ \\
\hline 5 & 3 & 4 & $\geq 1$ & $\geq 5$ \\
\hline 6 & 4 & 7 & $\geq 2$ & $\geq 9$ \\
\hline 7 & 4 & 10 & $\geq 3$ & $\geq 13$ \\
\hline 8 & 5 & 14 & $\geq 4$ & $\geq 17$ \\
\hline
\end{tabular}

Os procedimentos descritos acima não fornecem testes completos para normalidade e linearidade. Existe a possibilidade de que um processo não normal tenha um bi-espectro igual a zero e um teste exaustivo deveria testar todos os espectros de altas ordens. Notamos pela expressão (5.4) que o bi-espectro $f_{x}\left(\omega_{1}, \omega_{2}\right)$ pode desaparecer para um modelo linear desde que $\beta=0$. De forma similar, a constância de $\gamma^{2}\left(\omega_{1}, \omega_{2}\right)$ não necessariamente implica que o processo segue um modelo linear. Entretanto, em situações práticas, desvios da normalidade ou linearidade podem aparecer no bi-espectro e esta é a base do teste acima. 


\subsection{Testes de Hinich}

Segundo Hinich (1982), o teste de linearidade de Subba Rao e Gabr (1980) descrito na Seção 5.1 é muito sensivel à presença de outliers quando os valores do espectro são muito pequenos. Além disso, a conclusão depende da escolha da janela espectral e da construção da grade de freqüências. Neste teste, Hinich sugere o uso da matriz de covariâncias assintóticas do bi-espectro amostral ao invés da matriz amostral de segundos momentos. O uso da matriz de covariâncias é razoável quando o tamanho amostral é grande.

Seja $\left(\mathrm{X}_{\left.0, \ldots, \mathrm{X}_{\mathrm{T}-1}\right)}\right.$ uma amostra aleatória de uma série temporal gerada por um processo estacionário de média zero. Vamos definir o seguinte estimador preliminar do bi-espectro para cada par de inteiros $(\mathrm{j}, \mathrm{k})$

$$
F(j, k)=\frac{1}{T} d^{T}\left(\omega_{j}\right) d^{T}\left(\omega_{k}\right)\left(d^{T}\left(\omega_{j+k}\right)\right)^{*}
$$

em que $d^{T}\left(\omega_{j}\right)=\sum_{t=0}^{T-1} X_{t} \exp \left(-i \omega_{j} t\right), \omega_{j}=\frac{2 \pi j}{T}$ para $j=0,1 \ldots, T-1$ e o símbolo * denotando o complexo conjugado.

Como $d^{T}\left(\omega_{j+T}\right)=d^{T}\left(\omega_{j}\right)$ e $d^{T}\left(\omega_{T-j}\right)=\left(d^{T}\left(\omega_{j}\right)\right)^{*}$ o domínio principal de $F(j, k)$ é a grade triangular dada por

$$
\mathrm{P}=\left\{0<\mathrm{j} \leq \frac{\mathrm{T}}{2}, \quad 0<\mathrm{k} \leq \mathrm{j}, \quad 2 \mathrm{j}+\mathrm{k} \leq \mathrm{T}\right\} \text {, para } \mathrm{T} \text { par. }
$$

Existem muitas maneiras de calcular uma média de $F(\mathrm{j}, \mathrm{k})$ em um subconjunto do conjunto $\mathrm{P}$ e obter um estimador consistente para o bi-espectro. Uma 
maneira simples é calcular a média de $\mathrm{F}(\mathrm{j}, \mathrm{k})$ em $\mathrm{M}^{2}$ pontos centrados em $\left((2 m-1) \frac{M}{2},(2 n-1) \frac{M}{2}\right)$.

Quando todos os pontos $(\mathrm{j}, \mathrm{k})$ utilizados para obter um estimador consistente para o bi-espectro estiverem no conjunto P, Hinich (1982) sugere para cada par $\left(m^{*}, n^{*}\right)$ o seguinte estimador consistente para o bi-espectro

$$
\hat{f}_{H}\left(m^{*}, n^{*}\right)=\frac{1}{M^{2}} \sum_{j=(m-1) M}^{m M-1} \sum_{k=(n-1) M}^{n M-1} F(j, k),
$$

em que $\mathrm{m}^{*}=\frac{2 \pi(2 \mathrm{~m}-1) \mathrm{M}}{2 \mathrm{~T}}, \mathrm{n}^{*}=\frac{2 \pi(2 \mathrm{n}-1) \mathrm{M}}{2 \mathrm{~T}}$ e $\mathrm{M}=\mathrm{T}^{\mathrm{c}}$ para $\frac{1}{2}<\mathrm{c}<1$.

$\mathrm{O}$ parâmetro $\mathrm{c}$ tem como objetivo controlar o equilibrio entre o vício e a variância do estimador $\hat{\mathrm{f}}_{\mathrm{H}}\left(\mathrm{m}^{*}, \mathrm{n}^{*}\right)$.

As estatísticas utilizadas nos teste de linearidade e normalidade, que serão descritos a seguir, devem ser calculadas nos pares de freqüências definidos pelo seguinte sub-conjunto do conjunto $\mathrm{P}$

$$
\mathrm{L}=\left\{(2 \mathrm{~m}-1) \frac{\mathrm{M}}{2},(2 \mathrm{n}-1) \frac{\mathrm{M}}{2} \quad \text { para } \mathrm{m}=1, \ldots, \mathrm{n} \text { e } \mathrm{m} \leq \frac{\mathrm{T}}{2 \mathrm{M}}-\frac{\mathrm{n}}{2}+\frac{3}{4}\right\} .
$$

Na Tabela 5.3 estão os pares de freqüências definidos pelo conjunto L para $\mathrm{T}=100, \mathrm{c}=0,6$ e $\mathrm{M}=16$. 
Tabela 5.3 - Pares de freqüências definidos pelo conjunto $\underline{\mathrm{L}}$ para $\mathrm{T}=100, \mathrm{c}=0,6$ e $\mathrm{M}=16$

\begin{tabular}{cccc}
\hline $\mathrm{m}$ & $\mathrm{n}$ & $\mathrm{m}^{*}$ & $\mathrm{n}^{*}$ \\
\hline 1 & 1 & 0,503 & 0,503 \\
1 & 2 & 0,503 & 1,508 \\
1 & 3 & 0,503 & 2,513 \\
1 & 4 & 0,503 & 3,519 \\
1 & 5 & 0,503 & 4,524 \\
1 & 6 & 0,503 & 5,529 \\
2 & 2 & 1,508 & 1,508 \\
2 & 3 & 1,508 & 2,513 \\
2 & 4 & 1,508 & 3,519 \\
\hline
\end{tabular}

Hinich (1982) sugere como estimador do espectro o seguinte estimador suavizado de periodogramas

$$
\hat{\mathrm{f}}_{\mathrm{H}}\left(\omega_{\mathrm{k}}\right)=\frac{1}{2 \mathrm{~d}+1} \sum_{\mathrm{j}=-\mathrm{d}}^{\mathrm{d}} \hat{\mathrm{f}}\left(\omega_{\mathrm{k}+\mathrm{j}}\right)
$$

em que $\hat{f}\left(\omega_{k}\right)=\frac{1}{4 \pi} I_{n}\left(\omega_{k}\right)$ e $I_{n}\left(\omega_{k}\right)=\frac{2}{T} \sum_{t=1}^{T} \sum_{s=1}^{T} X_{t} X_{s} \exp \left(i \omega_{k}(t-s)\right)$.

\subsubsection{Teste de normalidade}

Este teste é baseado na seguinte ponderação da raiz quadrada da bicoerência

$$
\hat{\gamma}_{\mathrm{m}^{*}, \mathrm{n}^{*}}=\frac{\hat{\mathrm{f}}_{\mathrm{H}}\left(\mathrm{m}^{*}, \mathrm{n}^{*}\right)}{\left(\mathrm{T}^{1-4 \mathrm{c}} \mathrm{Q}_{\mathrm{m}^{*}, \mathrm{n}^{*}}\right)^{0,5}\left[\hat{\mathrm{f}}_{\mathrm{H}}(\mathrm{m} *) \hat{\mathrm{f}}_{\mathrm{H}}(\mathrm{n} *) \hat{\mathrm{f}}_{\mathrm{H}}\left(\mathrm{m}^{*}+\mathrm{n} *\right)\right]}
$$

em que $\hat{f}_{H}\left(m^{*}, n^{*}\right)$ é o estimador da função densidade bi-espectral, dado por (5.26), $\hat{\mathrm{f}}_{\mathrm{H}}\left(\mathrm{m}^{*}\right)$ é o estimador da função densidade espectral, dado por (5.28) e 
$\mathrm{Q}_{\mathrm{m}^{*}, \mathrm{n}^{*}}$ é o número de pares $(\mathrm{j}, \mathrm{k})$ que estão no conjunto $\mathrm{P}$ e estão fora das bordas $(\mathrm{j}=\mathrm{k}$ e $2 \mathrm{j}+\mathrm{k}=\mathrm{T})$ mais duas vezes o número de pares nas bordas.

\section{Propriedades do estimador $\hat{\gamma}_{\mathrm{m}^{*}, \mathrm{n}^{*}}$}

i) $\hat{\gamma}_{\mathrm{m}^{*}, \mathrm{n}^{*}} \stackrel{\mathrm{D}}{\longrightarrow} \mathrm{N}^{\mathrm{c}}(\mu, 1)$, em que $\mu=\sqrt{\lambda_{\mathrm{m}^{*}, \mathrm{n}^{*}}}$ e $\lambda_{\mathrm{m}^{*}, \mathrm{n}^{*}}=2\left(\mathrm{~T}^{1-4 \mathrm{c}} \mathrm{Q}_{\mathrm{m}^{*}, \mathrm{n}^{*}}\right)^{-1} \gamma^{2}\left(\mathrm{~m}^{*}, \mathrm{n}^{*}\right)$;

ii) $2\left|\hat{\gamma}_{\mathrm{m}^{*}, \mathrm{n}^{*}}\right|^{2} \stackrel{\mathrm{D}}{\longrightarrow} \chi_{2}^{2}\left(\lambda_{\mathrm{m}^{*}, \mathrm{n}^{*}}\right)$;

iii) $\lambda_{\mathrm{m}^{*}, \mathrm{n}^{*}} \geq 2 \mathrm{~T}^{2 \mathrm{c}-1} \gamma^{2}\left(\mathrm{~m}^{*}, \mathrm{n}^{*}\right)$;

iv) $2 \sum_{\left(\mathrm{m}^{*}, \mathrm{n}^{*}\right) \in \mathrm{L}}\left|\hat{\gamma}_{\mathrm{m}^{*}, \mathrm{n}^{*}}\right|^{2} \stackrel{\mathrm{D}}{\longrightarrow} \chi_{2 \mathrm{P} 1}^{2}(\lambda)$, em que $\lambda=\sum_{\left(\mathrm{m}^{*}, \mathrm{n}^{*}\right) \in \mathrm{L}} \lambda_{\mathrm{m}^{*}, \mathrm{n}^{*}}$ e P1 é o número de pares $\left(\mathrm{m}^{*}, \mathrm{n}^{*}\right)$ no conjunto $\mathrm{L}$, que é aproximadamente $\frac{\mathrm{T}^{2}}{12 \mathrm{M}^{2}}$

v)Quando $f_{x}\left(\omega_{1}, \omega_{2}\right) \equiv 0$ a estatística $S$ dada por

$$
\mathrm{S}=2 \sum_{\mathrm{m}^{*}} \sum_{\mathrm{n}^{*}}\left|\gamma_{\mathrm{m}^{*}, \mathrm{n}^{*}}\right|^{2}, \text { para }\left(\mathrm{m}^{*}, \mathrm{n}^{*}\right) \in \mathrm{L}
$$

possui distribuição assintótica $\chi_{2 \mathrm{Pl}}^{2}$;

vi) $\mathrm{E}\left(\left|\hat{\gamma}_{\mathrm{m}^{*}, \mathrm{n}^{*}}\right|^{2}\right)=1+\lambda_{\mathrm{m}^{*}, \mathrm{n}^{*}} / 2$

vii) $\chi_{2}^{2}\left(\hat{\lambda}_{0}\right) \underset{\mathrm{T} \rightarrow \infty}{\mathrm{D}} \chi_{2}^{2}\left(\lambda_{0}\right)$, em que $\quad \hat{\lambda}_{0}=2\left(\mathrm{P}^{2} \mathrm{M}^{2}\right)^{-1} \sum_{\left(\mathrm{m}^{*}, \mathrm{n}^{*}\right) \in \mathrm{L}} \mathrm{Q}_{\mathrm{m}^{*}, \mathrm{n}^{*}}\left(\left|\hat{\gamma}_{\mathrm{m}^{*}, \mathrm{n}^{*}}\right|^{2}-1\right)$ e $\lambda_{0}=2\left(\mathrm{~T}^{2 \mathrm{c}-1}\right) \gamma^{2}\left(\mathrm{~m}^{*}, \mathrm{n}^{*}\right)$.

Para mais detalhes, ver Hinich (1982). 
Para testar a hipótese

$$
\mathrm{H}_{0}: \mathrm{f}_{\mathrm{x}}\left(\omega_{1}, \omega_{2}\right) \equiv 0 \text {, }
$$

Hinich sugeriu a estatística

$$
\hat{\mathrm{S}}=2 \sum_{\mathrm{m}^{*}} \sum_{\mathbf{n}^{*}}\left|\hat{\gamma}_{\mathrm{m}^{*}, \mathrm{n}^{*}}\right|^{2} \text { para } \mathrm{m}^{*}, \mathrm{n}^{*} \in \mathrm{L}
$$

A hipótese de normalidade é rejeitada se $\hat{\mathrm{S}}>\mathrm{t}_{\alpha}$ em que $\alpha=\mathrm{P}\left(\chi_{2 \mathrm{P} 1}^{2}>\mathrm{t}_{\alpha}\right)$. Quando a hipótese nula não for rejeitada o processo poderá não possuir distribuição normal mas os dados serão consistentes com um bi-espectro identicamente igual a zero.

\subsubsection{Teste de linearidade}

Hinich propôs um teste de linearidade não paramétrico baseado no intervalo interquartil, que é uma medida robusta de dispersão, pois o teste $\mathrm{F}$ pode ser sensível a outliers em $\hat{\gamma}_{\mathrm{m}^{*}, \mathrm{n}^{*}}$, dado por (5.29), para pequenas estimativas do espectro em certas freqüências. O teste proposto usa o intervalo interquartil, de um sub-conjunto de $\left\{2\left|\hat{\gamma}_{\mathrm{m}^{*}, \mathrm{n}^{*}}\right|^{2}\right\}$, que é robusto para outliers.

Seja R a amplitude interquartil amostral de $2\left|\hat{\gamma}_{\mathrm{m}^{*}, \mathrm{n}^{*}}\right|^{2}$ para pares em P (quando $\mathrm{Q}_{\mathrm{m}^{*}, \mathrm{n}^{*}}=\mathrm{M}^{2}$ ).

Rejeitamos a hipótese de linearidade se $\mathrm{R}$ for significantemente maior ou menor que a amplitude interquartil de $\chi_{2}^{2}\left(\hat{\lambda}_{0}\right)$ em que $\hat{\lambda}_{0}$ é um estimador consistente de $\lambda_{0}=2 \mathrm{~T}^{2 \mathrm{c}-1} \gamma_{\mathrm{m}^{*}, \mathrm{n}^{*}}$. 
Segundo Hinich (1982), das propriedades (iii) e (vi) e das propriedades assintóticas de $\hat{\gamma}_{\mathrm{m}^{*}, \mathrm{n}^{*}}$ temos que

$$
\hat{\lambda}_{0}=2\left(\mathrm{P}^{2} \mathrm{M}^{2}\right)^{-1} \sum_{\left(\mathrm{m}^{*}, \mathrm{n}^{*}\right) \in \mathbf{L}} \mathrm{Q}_{\mathrm{m}^{*}, \mathrm{n}^{*}}\left(\left|\hat{\gamma}_{\mathrm{m}^{*}, \mathrm{n}^{*}}\right|^{2}-1\right)
$$

$\mathrm{O}$ verdadeiro intervalo interquartil da densidade $\chi_{2}^{2}\left(\lambda_{0}\right)$ é $\xi_{3}-\xi_{1}$ em que $\xi_{1}$ e $\xi_{3}$ são respectivamente o $1^{\circ}$ e o $3^{\circ}$ quartil da densidade $\chi_{2}^{2}\left(\lambda_{0}\right)$, isto é, $\mathrm{P}\left(\chi_{2}^{2}\left(\lambda_{0}\right)<\xi_{1}\right)=\mathrm{P}\left(\chi_{2}^{2}\left(\lambda_{0}\right)>\xi_{3}\right)=0,25$.

Seja $\mathrm{P}_{2}$ o número total de pares em $\mathrm{P}$. Quando $\mathrm{P}_{2} \rightarrow \infty, \mathrm{R}$ possui distribuição aproximadamente $N\left(\xi_{3}-\xi_{1}, \sigma_{0}^{2}\right)$,em que $\sigma_{0}^{2}=(16 \mathrm{P} 2)^{-1}\left[3\left(\xi_{1}\right)^{2}-2\left(\xi_{1}\right)\left(\xi_{3}\right)+3\left(\xi_{3}\right)^{2}\right]$.

Na prática o estimador $\hat{\lambda}_{0}$ dado por (5.31) é utilizado no cálculo dos valores de $\xi_{1}, \xi_{3}, \mathrm{f}\left(\xi_{1}\right)$ e $\mathrm{f}\left(\xi_{3}\right)$ para determinar a região crítica para $\mathrm{R}$.

Ashley, Hinich and Patterson (1986) e Chan e Tong (1986) estudaram diferentes simulações para determinar o tamanho amostral e o poder dos testes de linearidade e normalidade e chegaram às seguintes conclusões:

- Para amostras com tamanho inferiores a 256 observações os testes não são consistentes;

- O poder do teste aumenta com o tamanho amostral;

- O valor ótimo para o ponto de truncamento $\mathrm{M}$ é aproximadamente igual a $0,7 \sqrt{\mathrm{T}}$, em que T é o número de observações da série;

- O teste de linearidade deve ser aplicado como um teste para adequação de um modelo linear para uma série empírica; 
- Para um processo não estacionário, a hipótese nula de linearidade pode não ser rejeitada indevidamente (o argumento oposto não é verdadeiro). Porém esta não estacionariedade não permite a detecção de ciclos de baixa freqüência para um estudo gráfico.

\subsection{Testes de Brillinger e Irizarry}

Brillinger e Irizarry (1998) também propuseram testes, para detectar a linearidade e a normalidade de uma série temporal, baseados no bi-espectro.

Descreveremos a seguir o procedimento utilizado por Brillinger e Irizarry para estimar o espectro, o bi-espectro e a bi-coerência de uma série temporal estacionária com média zero.

Inicialmente a série temporal com $\mathrm{T}$ pontos é particionada em $\mathrm{L}$ trechos contendo $\mathrm{V}$ observações cada um, de tal forma que $\mathrm{T}=\mathrm{LV}$. Após a partição, aplicamos um filtro ("taper") e, então, calculamos a transformada de Fourier da série transformada e o periodograma de terceira ordem dados por

$$
\mathrm{d}^{\mathrm{v}}\left(\omega_{1} ; \ell\right)=\sum_{\mathrm{v}=0}^{\mathrm{V}-1} \mathrm{~h}\left(\frac{\mathrm{v}+1}{\mathrm{~V}+1}\right) \mathrm{X}_{\ell \mathrm{v}+\mathrm{v}} \exp \left(-\mathrm{iv} \omega_{1}\right)
$$

e

$$
I^{v}\left(\omega_{1}, \omega_{2} ; \ell\right)=\frac{1}{(2 \pi)^{2} h_{3}} d^{v}\left(\omega_{1} ; \ell\right) d^{v}\left(\omega_{2} ; \ell\right)\left(d^{v}\left(\omega_{1}+\omega_{2} ; \ell\right)\right)^{*}
$$

respectivamente, em que $h(u)=\frac{1}{2}(1-\cos (2 \pi u)), h_{3}=\int h(u)^{3} d u$ e o símbolo * denotando o complexo conjugado. 
O estimador do bi-espectro é um estimador suavizado de periodogramas de terceira ordem, dado por

$$
\hat{\mathrm{f}}_{\mathrm{B}}\left(\omega_{1}, \omega_{2}\right)=\frac{1}{\mathrm{~L}} \sum_{\ell=0}^{\mathrm{L}-1} \mathrm{I}^{\mathrm{v}}\left(\omega_{1}, \omega_{2} ; \ell\right)
$$

Um estimador da função densidade espectral da série temporal estacionária, é obtido pelo mesmo procedimento utilizado para obter a estimativa do biespectro, isto é,

$$
\hat{\mathrm{f}}_{\mathrm{B}}\left(\omega_{1}\right)=\frac{1}{\mathrm{~L}} \sum_{\ell=0}^{\mathrm{L}-1} \mathrm{I}^{\mathrm{V}}\left(\omega_{1} ; \ell\right)
$$

em que

$$
\mathrm{I}^{\mathrm{v}}\left(\omega_{1} ; \ell\right)=\frac{1}{2 \pi \mathrm{h}_{2}}\left|\mathrm{~d}^{\mathrm{v}}\left(\omega_{1}, \ell\right)\right|^{2}
$$

$e h_{2}=\int h(u)^{2} d u$

Brillinger e Irizarry sugerem o seguinte estimador para a bi-coerência

$$
\hat{\gamma}_{B}^{2}\left(\omega_{1}, \omega_{2}\right)=\frac{\left|\hat{f}_{B}\left(\omega_{1}, \omega_{2}\right)\right|^{2}}{\hat{f}_{B}\left(\omega_{1}\right) \hat{f}_{B}\left(\omega_{2}\right) \hat{f}_{B}\left(\omega_{1}+\omega_{2}\right)} .
$$




\section{Propriedades do estimador do bi-espectro e da bi-coerência}

i)Fora das bordas do domínio fundamental, ou seja, para $0<\omega_{2} \leq \omega_{1}$, e $\omega_{1}+\frac{\omega_{2}}{2}<\pi, \hat{\mathrm{f}}_{\mathrm{B}}\left(\omega_{1}, \omega_{2}\right) \stackrel{\mathrm{D}}{\longrightarrow} \mathrm{N}^{\mathrm{c}}\left(\mathrm{f}_{\mathrm{N}}\left(\omega_{1}, \omega_{2}\right), \mathrm{V}_{1}\right)$ quando $\mathrm{L} \rightarrow \infty, \mathrm{V} \rightarrow \infty$ e $\frac{\mathrm{V}}{\mathrm{L}} \rightarrow 0$, em que $V_{1}=\frac{h_{6}}{h_{3}^{2}} \frac{1}{2 \pi} f_{x}\left(\omega_{1}\right) f_{x}\left(\omega_{2}\right) f_{x}\left(\omega_{1}+\omega_{2}\right) \frac{V}{L}$ e $h_{k}=\int h(u)^{k} d u$;

ii)Estimadores em freqüências distintas são assintoticamente independentes;

iii)Sob a hipótese de que $\mathrm{f}_{\mathrm{x}}\left(\omega_{1}, \omega_{2}\right)=0$, temos que $\left|\hat{f}_{\mathrm{B}}\left(\omega_{1}, \omega_{2}\right)\right|^{2} \stackrel{\mathrm{D}}{\longrightarrow} \mathrm{a} \frac{\chi_{2}^{2}}{2}$, em que $a=\frac{h_{6}}{h_{3}^{2}} \frac{1}{2 \pi} f_{x}\left(\omega_{1}\right) f_{x}\left(\omega_{2}\right) f_{x}\left(\omega_{1}+\omega_{2}\right) \frac{V}{L}$ e $h_{k}=\int h(u)^{k} d u ;$

iv)Se $f_{x}\left(\omega_{1}, \omega_{2}\right) \neq 0$ temos que $\left|\hat{f}_{B}\left(\omega_{1}, \omega_{2}\right)\right|^{2} \stackrel{D}{\longrightarrow} N\left(\left|f_{x}\left(\omega_{1}, \omega_{2}\right)\right|^{2}, V_{2}\right)$, em que $V_{2}=$ $2 \frac{h_{6}}{h_{3}^{2}} \frac{V}{L} \frac{1}{2 \pi} f_{x}\left(\omega_{1}\right) f_{x}\left(\omega_{2}\right) f_{x}\left(\omega_{1}+\omega_{2}\right)\left|f_{x}\left(\omega_{1}, \omega_{2}\right)\right|^{2}$ e $h_{k}=\int h(u)^{k} d u ;$

v)Se $f_{x}\left(\omega_{1}, \omega_{2}\right)=0$ temos que $\hat{\gamma}_{B}{ }^{2}\left(\omega_{1}, \omega_{2}\right) \stackrel{D}{\longrightarrow} \exp (\lambda)$, em que $\exp (\lambda)$ é uma variável aleatória com distribuição exponencial de média $\left(\frac{\mathrm{h}_{6}}{\mathrm{~h}_{3}^{2}} \frac{\mathrm{V}}{\mathrm{L}} \frac{1}{2 \pi}\right)$;

vi)Se $f_{\mathrm{x}}\left(\omega_{1}, \omega_{2}\right) \neq 0$ temos que $\hat{\gamma}_{\mathrm{B}}{ }^{2}\left(\omega_{1}, \omega_{2}\right) \stackrel{\mathrm{D}}{\longrightarrow} \mathrm{N}\left(\gamma^{2}\left(\omega_{1}, \omega_{2}\right), \mathrm{V}_{3}\right)$, em que $\mathrm{V}_{3}=2 \frac{\mathrm{h}_{6}}{\mathrm{~h}_{3}^{2}} \frac{\mathrm{V}}{\mathrm{L}} \frac{1}{2 \pi} \frac{\left|\mathrm{f}_{\mathrm{x}}\left(\mathrm{w}_{1}, \mathrm{w}_{2}\right)\right|^{2}}{\mathrm{f}_{\mathrm{x}}\left(\mathrm{w}_{1}\right) \mathrm{f}_{\mathrm{x}}\left(\mathrm{w}_{2}\right) \mathrm{f}_{\mathrm{x}}\left(\mathrm{w}_{1}+\mathrm{w}_{2}\right)}$ e $h_{\mathrm{k}}=\int \mathrm{h}(\mathrm{u})^{\mathrm{k}} \mathrm{du} ;$

vii)O estimador $\hat{\gamma}_{\mathrm{B}}\left(\omega_{1}, \omega_{2}\right) \stackrel{\mathrm{D}}{\longrightarrow} \mathrm{N}\left(\gamma\left(\omega_{1}, \omega_{2}\right), \mathrm{V}_{4}\right)$, em que $\mathrm{V}_{4}=\frac{1}{2} \frac{\mathrm{h}_{6}}{\mathrm{~h}_{3}^{2}} \frac{\mathrm{V}}{\mathrm{L}} \frac{1}{2 \pi}$ e $h_{k}=\int h(u)^{k} d u$ 
viii)Quando o processo $X_{t}$ é reversível, ou seja, quando as propriedades de $X_{t}$ e $\mathrm{X}_{-t}$ são as mesmas, a parte imaginária do bi-espectro é identicamente nula, ver Brillinger e Rosenblatt (1967).

Para mais detalhes, ver Brillinger e Irizarry (1998).

\subsubsection{Teste de normalidade}

No teste de normalidade em que o objetivo é testar a hipótese

$$
\mathrm{H}_{01}: \mathrm{f}_{\mathrm{x}}\left(\omega_{1}, \omega_{2}\right)=0 \text {, }
$$

Brillinger e Irizarry utilizam o estimador da bi-coerência dado por (5.37), calculado nos pares de freqüência $\left(\omega_{1}, \omega_{2}\right)$ que satisfazem

$$
0 \leq \omega_{2} \leq \omega_{1} \text { e } \omega_{1}+\frac{\omega_{2}}{2} \leq \pi .
$$

Pela propriedade v) temos que, sob $\mathrm{H}_{01}$,

$$
\hat{\gamma}_{\mathrm{B}}{ }^{2}\left(\omega_{1}, \omega_{2}\right) \stackrel{\mathrm{D}}{\longrightarrow} \exp (\lambda),
$$

em que $\exp (\lambda)$ é uma variável aleatória com distribuição exponencial de média $\left(\frac{\mathrm{h}_{6}}{\mathrm{~h}_{3}^{2}} \frac{\mathrm{V}}{\mathrm{L}} \frac{\mathrm{l}}{2 \pi}\right)$.

Desta forma rejeitamos a hipótese de normalidade se o nível descritivo associado a $\hat{\gamma}_{B}{ }^{2}\left(\omega_{1}, \omega_{2}\right)$ for menor do que $5 \%$ para mais do que $5 \%$ dos pares de freqüência $\left(\omega_{1}, \omega_{2}\right)$ que satisfizerem (5.38). 


\subsubsection{Teste de linearidade}

Para o teste de linearidade, em que o objetivo é testar a hipótese,

$$
\mathrm{H}_{02}: \gamma^{2}\left(\omega_{1}, \omega_{2}\right)=\text { constante, }
$$

calcularemos para os pares de freqüências $\left(\omega_{i}, \omega_{j}\right)$, que satisfizerem (5.38), a estatística

$$
X^{*}\left(\omega_{i}, \omega_{j}\right)=\left(\frac{\hat{\gamma}_{B}\left(\omega_{i}, \omega_{j}\right)-\bar{\gamma}_{B}\left(\omega_{i}, \omega_{j}\right)}{\sigma_{X^{*}}}\right)^{2}
$$

em que $\bar{\gamma}_{B}\left(\omega_{i}, \omega_{j}\right)=\frac{\sum_{i} \sum_{j} \hat{\gamma}_{B}\left(\omega_{i}, \omega_{j}\right)}{n}$, n é o número de freqüências que satisfazem (5.38) e $\sigma_{X^{*}}=\sqrt[2]{\frac{h_{6}}{h_{3}^{2}} \frac{V}{L} \frac{1}{2 \pi}+\frac{h_{6}}{\mathrm{nh}_{3}^{2}} \frac{V}{L} \frac{1}{2 \pi}}$

Pela propriedade vii) temos que

$$
\mathrm{X}^{*}\left(\omega_{\mathrm{i}}, \omega_{\mathrm{j}}\right) \stackrel{\mathrm{D}}{\longrightarrow} \chi_{1}^{2}
$$

Desta forma rejeitamos a hipótese de linearidade se o nível descritivo associado a $X^{*}\left(\omega_{\mathrm{i}}, \omega_{\mathrm{j}}\right)$ for menor do que $5 \%$ para mais do que $5 \%$ dos pares de freqüência que satisfizerem (5.38). 


\section{Capítulo 6}

\section{Testes de linearidade no domínio do tempo}

Abordaremos, neste capítulo os testes paramétricos ou semi-paramétricos de McLeod e Li (1983) e Tsay (1986) e o teste não paramétrico de Poggi e Portier (1997).

\subsection{Teste de McLeod e Li}

$O$ teste descrito nesta seção foi motivado pelo fato de que se $X_{t}$ é uma série temporal estacionária e gaussiana então

$$
\rho_{\mathrm{x}_{\mathrm{t}}^{2}}(\mathrm{j})=\left(\rho_{\mathrm{x}_{\mathrm{t}}}(\mathrm{j})\right)^{2} \text {, para } \forall \mathrm{j}
$$

Segundo Granger e Andersen (1978) a não validade deste resultado tende a indicar possibilidade de não linearidade. Maravall (1983) considerou o uso de 
$\rho_{\hat{e}_{t}^{2}}(j)$, em que $\hat{e}_{t}$ são os resíduos de um modelo ajustado. McLeod e Li (1983) propuseram um teste do tipo "Portmanteau" baseado na autocorrelação amostral do quadrado dos resíduos, de uma série temporal estacionária e gaussiana, para testar a hipótese de linearidade.

A hipótese a ser testada é

$\mathrm{H}_{0}$ : a série é linear.

Sejam $\left(\hat{\mathrm{e}}_{1}, \ldots, \hat{\mathrm{e}}_{\mathrm{T}}\right)$ os resíduos ajustados de um modelo ARMA e $\hat{\rho}_{\mathrm{e}}$ a autocorrelação amostral do quadrado dos resíduos dada por

$$
\hat{\rho}_{e}(k)=\frac{\sum_{j=1}^{T-k}\left(\hat{e}^{2}{ }_{j}-\hat{\sigma}^{2}\right)\left(\hat{e}^{2}{ }_{j+k}-\hat{\sigma}^{2}\right)}{\sum_{j=1}^{n}\left(\hat{e}^{2}{ }_{j}-\hat{\sigma}^{2}\right)^{2}},
$$

em que $\hat{\sigma}^{2}=\frac{\sum_{j=1}^{T} \hat{e}_{j}^{2}}{T}$.

Para detectar um modelo ARMA mal especificado McLeod e Li propuseram a seguinte estatística

$$
\mathrm{Q}=\mathrm{T}(\mathrm{T}+2) \sum_{\mathrm{k}=1}^{\mathrm{m}} \frac{\hat{\rho}_{\mathrm{e}}^{2}(\mathrm{k})}{\mathrm{T}-\mathrm{k}}
$$

A estatística (6.1) pode expressar a possibilidade de detectar não linearidade de um modelo na direção de bilinearidade e sob a hipótese de que o 
modelo é linear, possui distribuição $\chi_{\mathrm{m}}^{2}$ se as verdadeiras inovações forem independentes.

A hipótese $\mathrm{H}_{0}$ deve ser rejeitada quando $\mathrm{Q}>\mathrm{q}$, em que q é o (1- $\alpha$ )-ésimo percentil da distribuição $\chi_{\mathrm{m}}^{2}$.

Segundo Tong (1990), não é claro se este teste distingue entre um modelo não-linear e um modelo ARMA mal ajustado, quando obtemos um resultado significante. Segundo Davies e Petruccelli (1985) e Luukkonen et al. (1988) este teste é adequado para testar a hipótese de que o modelo é linear contra a hipótese de que o modelo é não-linear do tipo $\mathrm{ARCH}$.

\subsection{Teste de Tsay}

Tsay (1986) propôs um teste de linearidade, para séries temporais estacionárias, aprimorando o teste de Keenan (1985) que é similar ao teste de Tukey para não aditividade (Tukey,1949).

A hipótese de interesse é

$$
H_{0}:\left(X_{t}-\mu\right)=\sum_{j=1}^{m} a_{j}\left(X_{t-j}-\mu\right)+e_{t},
$$

em que e é uma seqüência de variáveis aleatórias independentes e identicamente distribuídas com média zero e variância $\sigma^{2}$.

Sejam $X_{1}, \ldots, X_{T}$ observações de uma série temporal. O teste de Tsay é constituído dos seguintes passos: 
i) Ajustar uma regressão linear de $X_{t}$ sobre $\left(1, X_{t-1}, \ldots, X_{t-m}\right)$ em que $m$ é um número inteiro positivo fixado, isto é,

$$
\mathbf{X}_{\mathbf{t}}=\mathbf{W}_{\mathbf{t}} \phi+\mathbf{e}_{\mathbf{t}}
$$

em que $\mathbf{W}_{\mathbf{t}}=\left(1, \mathrm{X}_{\mathrm{t}-1}, \ldots, \mathrm{X}_{\mathrm{t}-\mathrm{m}}\right)$ e $\phi=\left(\phi_{0}, \ldots, \phi_{\mathrm{m}}\right)^{\prime}, \phi_{0}=1$

ii) Calcular os resíduos ajustados $\hat{\mathrm{e}}=\left(\hat{\mathrm{e}}_{\mathrm{m}+1}, \ldots, \hat{\mathrm{e}}_{\mathrm{T}}\right)^{\prime}$.

iii) Considere $\mathbb{Z}_{\mathbf{t}}$ o vetor linha $\mathrm{r}=\frac{\mathrm{m}(\mathrm{m}+1)}{2}$-dimensional, que é dado pelo vech ( $\mathbf{U}^{\mathbf{t}} \mathbf{U}$ ), ou seja, $\mathbf{Z}_{\mathbf{t}}$ é obtido por meio dos elementos situados sobre e abaixo da diagonal principal da matriz $\mathbf{U}^{\prime} \mathbf{U}$ em que $\mathbf{U}=\left(\mathrm{X}_{\mathbf{t}-1}, \ldots, X_{\mathbf{t}-\mathbf{m}}\right)$. Temos que $\mathbf{U}^{\prime} \mathbf{U}$ e $\mathbb{Z}_{\mathbf{t}}$ são dados por

$$
\mathbf{U}^{\prime} \mathbf{U}=\left[\begin{array}{cccc}
X_{t-1}^{2} & X_{t-1} X_{t-2} & \cdots & X_{t-1} X_{t-m} \\
X_{t-2} X_{t-1} & X_{t-2}^{2} & \cdots & X_{t-2} X_{t-m} \\
\vdots & \vdots & \vdots & \vdots \\
X_{t-m} X_{t-1} & X_{t-m} X_{t-2} & \cdots & X_{t-m}^{2}
\end{array}\right]
$$

e

$$
\mathbf{Z}_{t}=\left[X^{2}{ }_{t-1}, X_{t-2} X_{t-1}, \ldots, X_{t-m} X_{t-1}, X^{2}{ }_{t-2}, X_{t-3} X_{t-2}, \ldots, X_{t-m} X_{t-2}, \ldots, X_{t-m}^{2}\right] .
$$

iv) Fazer uma regressão múltipla do vetor $\mathbf{Z}_{\mathrm{t}}$ sobre $\left(1, X_{\mathrm{t}-1}, \ldots, X_{\mathrm{t}-\mathrm{m}}\right)$, isto é,

$$
\mathbf{Z}_{\mathbf{t}}=\mathbf{W}_{\mathbf{t}} \mathrm{H}+\xi_{\mathbf{t}}
$$

em que $\mathbf{W}_{\mathbf{t}}=\left(1, X_{t-1}, \ldots, X_{t-m}\right)$ e $\mathbf{H}=\left(\begin{array}{ccc}h_{1,1} & \cdots & h_{1, r} \\ \vdots & \vdots & \vdots \\ h_{m, 1} & \cdots & h_{m, r}\end{array}\right)$, 
denotando o vetor de resíduos por

$$
\hat{\xi}=\left(\begin{array}{ccc}
\hat{\xi}_{\mathrm{m}+1,1} & \cdots & \hat{\xi}_{\mathrm{m}+1, \mathrm{r}} \\
\vdots & \vdots & \vdots \\
\hat{\xi}_{\mathrm{T}, 1} & \cdots & \hat{\xi}_{\mathrm{T}, \mathrm{r}}
\end{array}\right)=\left(\hat{\xi}_{1}, \ldots, \hat{\xi}_{\mathrm{r}}\right)
$$

v) Fazer uma regressão de $\hat{\mathbf{e}}$ sobre $\hat{\xi}$ e obter os resíduos do modelo que serão denotados por $\hat{\mathbf{a}}=\left(\hat{\mathrm{a}}_{\mathrm{m}+1}, \ldots, \hat{\mathrm{a}}_{\mathrm{T}}\right)^{\prime}$.

A estatística do teste é dada por

$$
F=\frac{\left(\sum_{t=m+1}^{T} \hat{e}_{t}^{\prime} \hat{\xi}_{t}\right)\left(\sum_{t=m+1}^{T} \hat{\xi}_{t}^{\prime} \hat{\xi}_{t}\right)^{-1}\left(\sum_{t=m+1}^{T} \hat{\xi}_{t}^{\prime} \hat{e}_{t}\right)(T-m-r-1)}{r \sum_{t=m+1}^{T} \hat{a}_{t}^{2}}
$$

ou, pela forma matricial,

$$
J=\frac{\left(\hat{e}^{\prime} \hat{\xi}\right)\left(\hat{\xi}^{\prime} \hat{\xi}\right)^{-1}\left(\hat{\xi}^{\prime} \hat{\mathrm{e}}\right)}{\mathrm{râ}^{\prime} \hat{\mathrm{a}}}(\mathrm{T}-\mathrm{m}-\mathrm{r}-1)
$$

Tsay (1986) provou que, sob a hipótese (6.2), a estatística (6.8) possui distribuição assintótica $\mathrm{F}\left(\frac{\mathrm{m}(\mathrm{m}+1)}{2}, \mathrm{~T}-\frac{\mathrm{m}(\mathrm{m}+3)}{2}-1\right)$.

O teste de linearidade proposto por Tsay possui a vantagem de ser de fácil implementação envolvendo pequena escolha subjetiva dos parâmetros, m sendo único. Este teste possui as seguintes desvantagens:

a) Provavelmente menos potente para detectar expansões da forma 
$H_{1}: X_{t}=\sum a_{j} e_{t-j}+\sum \sum \sum a_{j k l} e_{t-j} e_{t-k} e_{t-1}, \ldots$

pois o caminho é implícito em testar $\mathrm{H}_{0}$, dada por (6.2), na direção de

$H_{1}: X_{t}=\sum a_{j} e_{t-j}+\sum \sum a_{j k} e_{t-j} e_{t-k}$.

b) Possui um questionável resultado quando o verdadeiro modelo não-linear necessita de lags muito grandes no poder de expansão para alguns moderados graus de acurácia, por exemplo um modelo SETAR.

\subsection{Teste de Poggi e Portier}

Poggi e Portier (1997) propuseram um teste de linearidade não paramétrico no domínio do tempo que descreveremos a seguir.

Vamos considerar o seguinte modelo auto-regressivo funcional, assintoticamente estacionário de ordem $\mathrm{p}$, dado por

$$
X_{1}=f\left(X_{t-1}, \ldots, X_{t-p}\right)+e_{t} \quad t \in N
$$

em que $\mathrm{e}_{t}$ é um processo ruído branco com média zero e matriz de covariância $\Gamma, \mathrm{f}$ é uma função desconhecida de $\mathrm{R}^{\mathrm{p}} \rightarrow \mathrm{R}$ e $\mathrm{p}$ é a ordem conhecida do modelo.

Seja $X_{t}^{(p)}=\left(X_{t}, X_{t-1}, \ldots, X_{t-p+1}\right)$ um vetor de dimensão $R^{p}$. Vamos supor que o estado inicial $\mathrm{X}_{0}^{(\mathrm{p})}$ é dado e é independente de e. Segundo o resultado de Duflo (1990), o processo $X^{(p)}=\left(X_{t}^{(p)}\right)_{t \geq 0}$ é assintoticamente estacionário e possui distribuição invariante $\mu$, com momento de ordem $\mathrm{m}$ e função densidade de probabilidade $h$. 
Para estimar a função $f$ será utilizada uma estimativa de mínimos quadrados e uma estimativa não paramétrica baseada no "método kernel" (Rosenblatt,1956). A estimativa de mínimos quadrados é adequada quando a função $f$ é linear da forma $\mathrm{f}(\mathrm{x})=\mathbf{A x}$ e é dada pela solução da equação

$$
\left(\sum_{k=0}^{T-1} X_{k}^{(p)^{\prime}} X_{k}^{(p)}\right) \hat{A}_{n}=\sum_{k=0}^{T-1} X_{k}^{(p)^{\prime}} X_{k+1}
$$

ou seja,

$$
\hat{A}_{n}=\left(\sum_{k=0}^{T-1} X_{k}^{(p)^{\prime}} X_{k}^{(p)}\right)^{-1} \sum_{k=0}^{T-1} X_{k}^{(p)^{\prime}} X_{k+1}
$$

Quando a matriz $\left(\sum_{\mathrm{k}=0}^{\mathrm{T}-1} \mathrm{X}_{\mathrm{k}}^{(\mathrm{p})^{\prime}} \mathrm{X}_{\mathrm{k}}^{(\mathrm{p})}\right)$ não for invertivel podemos obter a estimativa de mínimos quadrados por meio da solução das equações:

$$
\hat{A}_{n+1}=\hat{A}_{n}+S_{n}^{-1} X_{n}^{(p)^{\prime}}\left(X_{n+1}-\hat{A}_{n}^{\prime} X_{n}^{(p)}\right) \quad \operatorname{com} \hat{A}_{0}=0
$$

e

$$
S_{n}=S_{n-1}+X_{n}^{(p)^{\prime}} X_{n}^{(p)} \quad \operatorname{com~S}_{-1} \geq \lambda I_{p} \text { e } \lambda>0
$$

em que $I_{p}$ é uma matriz identidade de ordem p e $\lambda$ uma constante.

De acordo com Poggi e Portier (1997) a estimativa "kernel regression" da função $f$ e da função densidade de probabilidade $h$, para cada $x \in \mathbb{R}^{p}$, são dadas por

$$
\hat{f}_{n}(x)=\frac{\sum_{i=0}^{T-1} i^{a p} K\left(i^{a}\left(X_{i}^{(p)}-x\right)\right) X_{i+1}}{\sum_{i=0}^{T-1} i^{a p} K\left(i^{a}\left(X_{i}^{(p)}-x\right)\right)}
$$




$$
\hat{h}_{n}(x)=\frac{1}{T} \sum_{i=0}^{T-1} i^{a p} K\left(i^{a}\left(X_{i}^{(p)}-x\right)\right)
$$

em que a é uma constante que assume o valor $\frac{1}{p+1}$ para quando $p>1$ e $\frac{2}{5}$ quando $\mathrm{p}=1$ e $\mathrm{K}($.) é uma função densidade de probabilidade. A função densidade de probabilidade $K$ satisfaz as condições $\int K(y) d y=1$ e $\int\|y\| K(y) d y<\infty$. Temos que $\hat{f}_{n}(x)=0$ quando $\hat{h}_{n}(x)=0$.

Dados $\mathrm{x}_{1}, \ldots, \mathrm{x}_{\mathrm{q}}$ distintos pontos do suporte da distribuição estacionária $\mu$, a estatística de não linearidade é definida por

$$
s_{q}(n)=\sum_{i=1}^{q} \hat{h}_{n}\left(x_{i}\right)\left\|\hat{f}_{n}\left(x_{i}\right)-\hat{A}_{n} \cdot x_{i}\right\|_{(\Gamma)^{-1}}^{2},
$$

em que $\|y\|_{(\Gamma)^{-1}}^{2}=y^{\prime}(\Gamma)^{-1} y$.

Quando a distribuição estacionária $h$ for gaussiana e tivermos $p=1$, os $q$ pontos do suporte, $\mathrm{x}_{1}, \ldots, \mathrm{x}_{\mathrm{q}}$, podem ser escolhidos de forma equidistante no intervalo $\left[-1,25 \hat{\sigma}_{x} ; 1,25 \hat{\sigma}_{x}\right] \operatorname{com}\left|x_{i+1}-x_{i}\right|=3 \hat{\sigma}_{x} b_{n}$ em que $\hat{\sigma}_{x}$ é o desvio padrão das observações da série $X_{1}$ e $b_{n}=T^{-a}$. Uma segunda opção é escolher $x_{1}, \ldots, x_{q}$ por meio do histograma. Para $\mathrm{p}>1$ devem ser escolhidos os pontos, $\mathrm{x}_{1}, \ldots, \mathrm{x}_{\mathrm{q}}, \mathrm{em}^{\mathrm{p}} \mathrm{R}^{\mathrm{p}}$ tal que a função densidade $\mathrm{h}$ em cada ponto deve ser alta e a função

$$
\mathrm{D}\left(\mathrm{x}_{\mathrm{i}}, \mathrm{x}_{\mathrm{j}}\right)=\frac{1}{\mathrm{~T}} \sum_{\mathrm{k}=1}^{\mathrm{T}-1} \mathrm{~b}_{\mathrm{k}}^{-1} \mathrm{~K}\left(\mathrm{~b}_{\mathrm{k}}^{-1}\left(\mathrm{X}_{\mathrm{k}}^{\mathrm{p}}-\mathrm{x}_{\mathrm{i}}\right)\right) \mathrm{K}\left(\mathrm{b}_{\mathrm{k}}^{-1}\left(\mathrm{X}_{\mathrm{k}}^{\mathrm{p}}-\mathrm{x}_{\mathrm{j}}\right)\right),
$$

deve convergir para zero para $\mathrm{x}_{\mathrm{i}} \neq \mathrm{x}_{\mathrm{j}}$. 
Existem duas idéias por trás da escolha da estatística de não linearidade, dada pela expressão (6.16). A primeira é comparar as duas estimativas da função f, a "kernel regression" $\hat{f}_{n}(x)$ que é adequada para qualquer função $f$ e a estimativa de mínimos quadrados Â x que é adequada quando o modelo for linear. A segunda é baseada na escolha de q distintos pontos do suporte de $\mu$, nos quais calculamos o desvio quadrado entre as estimativas, ponderado pela densidade estimada $\hat{h}_{n}(x)$ nestes pontos. A soma destes desvios fornece um índice de não linearidade da função $f$, dada pela expressão (6.14).

Quando a variância do processo ruído branco, $\mathrm{e}_{\mathrm{t}}$, for desconhecida usaremos o seguinte estimador para $\Gamma$

$$
\hat{\Gamma}_{n}=\frac{1}{T}\left(\sum_{k=0}^{T-1}\left(X_{k+1}-\hat{A}_{n-1}^{\prime} X_{k}^{p}\right)^{\prime}\left(X_{k+1}-\hat{A}_{n-1}^{\prime} X_{k}^{p}\right)+\lambda\right),
$$

$\operatorname{com} \lambda>0$. O termo $\lambda /$ T assegura a invertibilidade da matriz $\hat{\Gamma}_{n}$.

Com o objetivo de testar a hipótese

$$
\begin{aligned}
& H_{0}: f(X)=a_{1} X_{t-1}+\ldots+a_{p} X_{t-p} \\
& H_{1}: f \text { é não-linear }
\end{aligned}
$$

utilizamos a estatística

$$
T_{q}(n)=\sum_{i=1}^{q} Z_{n}^{2}\left(x_{i}\right)
$$

em que 


$$
Z_{n}(x)=\left(\frac{T b_{n}^{p}(1+a p)}{\hat{\Gamma}_{n}\|K\|_{2}^{2}}\right)^{1 / 2}\left(\hat{h}_{n}(x)\right)^{1 / 2}\left(\hat{f}_{n}(x)-\hat{A}_{n}^{\prime} x\right)
$$

$$
\operatorname{com} b_{n}=T^{-a} \text { e } K(x)=\frac{1}{\left((2 \pi)^{1 / 2} \sigma_{x}\right)^{p}} \exp \left(\frac{-\|x\|^{2}}{2 \sigma_{x}^{2}}\right) \text { para } x \in R^{p}
$$

Sob $\mathrm{H}_{0}$, a estatística $\mathrm{T}_{\mathrm{q}}(\mathrm{n})$ possui distribuição assintótica $\chi^{2}(\mathrm{q})$.

O teste de linearidade de Poggi e Portier (1997) pode ser generalizado para o caso em que $\mathrm{d}>1$.

A estimativa "kernel regression" da função $f$ e da função densidade de probabilidade $h$, para cada $x \in R^{p}$, quando $d>1$ são dadas por

$$
\hat{f}_{n}(x)=\frac{\sum_{i=0}^{T-1} i^{\text {apd }} K\left(i^{a}\left(X_{i}^{(p)}-x\right)\right) X_{i+1}}{\sum_{i=0}^{T-1} i^{\text {apd }} K\left(i^{a}\left(X_{i}^{(p)}-x\right)\right)}
$$

$\mathrm{e}$

$$
\hat{h}_{n}(x)=\frac{1}{T} \sum_{i=0}^{T-1} i^{\text {apd }} K\left(i^{a}\left(X_{i}^{(p)}-x\right)\right)
$$

respectivamente.

Quando a variância do processo ruído branco for desconhecida usaremos o seguinte estimador para $\Gamma$

$$
\hat{\Gamma}_{n}=\frac{1}{T}\left(\sum_{k=0}^{T-1}\left(X_{k+1}-\hat{A}_{n-1}^{\prime} X_{k}^{p}\right)^{\prime}\left(X_{k+1}-\hat{A}_{n-1}^{\prime} X_{k}^{p}\right)+\lambda I_{d}\right)
$$


com $\lambda>0$. O termo $(\lambda / T) I_{d}$ assegura a invertibilidade da matriz $\hat{\Gamma}_{n}$.

A estatística do teste é dada por

$$
T_{q}(n)=T^{1-\text { adp }} \frac{1+a d p}{\|K\|_{2}^{2}} S_{q}(n),
$$

com $\mathrm{s}_{\mathrm{q}}(\mathrm{n})$ dado por (6.16).

Sob $\mathrm{H}_{0}$, a estatística $\mathrm{T}_{\mathrm{q}}(\mathrm{n})$ possui distribuição assintótica $\chi^{2}(\mathrm{dq})$.

\subsection{Outros testes de linearidade}

Podemos encontrar muitos outros testes de linearidade na literatura especializada. Dunis e Zhou (1998) sugerem um teste de linearidade não paramétrico no domínio da freqüência baseado no procedimento "bootstrap" e sugerem, também, um teste de linearidade paramétrico contra a hipótese de um modelo auto-regressivo exponencial. Para testar a linearidade contra a hipótese de um modelo bilinear pode ser utilizado o teste paramétrico de Keenan's (1985). 


\section{Capítulo 7}

\section{Aplicações}

\subsection{Introdução}

Neste Capítulo serão aplicados alguns dos testes de linearidade e normalidade descritos nos Capítulos 5 e 6 nas séries de poluição, temperatura e óbitos da cidade de São Paulo, ver Apêndice A. Os programas foram desenvolvidos em S-plus e em turbo pascal e podem ser encontrados no Apêndice B.

As séries utilizadas neste capítulo são descritas a seguir:

i) Total de óbitos por causas respiratórias para crianças de 1 a 11 meses (RESP) fornecido pelo Programa de Aprimoramento das Informações de Mortalidade no Município de São Paulo (PROAIM);

ii) Total de óbitos para crianças de 1 a 11 meses (TOT) fornecido pelo Programa de Aprimoramento das Informações de Mortalidade no Município de São Paulo (PROAIM); 
iii)Temperatura mínima $\left({ }^{\circ} \mathrm{C}\right)$ (TEMP) fornecida pelas estações da Companhia de Tecnologia de Saneamento Ambiental (CETESB) situadas no Parque D. Pedro e no Parque do Estado na Água Funda;

iv)Média da concentração do agente poluidor PM10 (PM10), material particulado, isto é, poeira composta de partículas menores do que 10 micra, calculada por meio das concentrações fornecidas por 11 estações da Companhia de Tecnologia de Saneamento Ambiental (CETESB) espalhadas pela cidade de São Paulo.

As séries descritas foram medidas diariamente no periodo de 1 de janeiro de 1996 a 31 de dezembro de 1997 com T=731 observações. A série de média da concentração de PM10 foi observada no período 18 de abril de 1996 a 31 de dezembro de 1997 com $\mathrm{T}=623$ observações diárias.

Para a aplicação dos testes de normalidade e linearidade de Brillinger e Irizarry (1998) devemos particionar a série de interesse em L subséries de tamanho $\mathrm{V}$ tal que $\mathrm{V}=\mathrm{T}^{\alpha}$ e $\mathrm{L}=\mathrm{T}^{1-\alpha}$, para $\alpha<0,5$. Desta forma as séries (1-B)TEMP, (1B)RESP e (1-B)TOT, que possuem 730 observações, foram particionadas em 46 subséries de tamanho $16(\alpha=0,421)$ e a série PM10 foi particionada em 38 subséries de tamanho $16(\alpha=0,431)$. O "taper" utilizado na obtenção do periodograma e do bi-espectro estimado é $\lambda(u)=\frac{1}{2}(1-\cos (2 \pi u))$.

Para a realização do teste de linearidade de Tsay (1986) é necessário a obtenção do parâmetro $\mathrm{m}$, que indica a quantidade de variáveis independentes da primeira regressão, para isto foram ajustados modelos auto-regressivos às séries e como valor de $\mathrm{m}$ tomamos a ordem de modelo auto-regressivo que melhor se ajustou aos dados; para esta escolha foram utilizados os seguintes critérios: 
- Critério de informação de Akaike

Segundo este critério o melhor modelo auto-regressivo para a série é aquele que possui o menor valor de $\operatorname{AIC}(\mathrm{k})$, sendo que

$$
\operatorname{AIC}(k)=\ln \hat{\sigma}^{2}(k)+\frac{2 k}{T}
$$

em que k é o número de parâmetros do modelo, T é o número de observação da série e $\hat{\sigma}^{2}(\mathrm{k})$ é a variância residual estimada do modelo auto-regressivo de ordem k.

- Critério de informação de Schwartz

Segundo este critério o melhor modelo auto-regressivo para a série é aquele que possui o menor valor de $\mathrm{SBC}$, sendo que

$$
\operatorname{SBC}(k)=\ln \hat{\sigma}^{2}(k)+\frac{k \ln (T)}{T},
$$

em que k é o número de parâmetros do modelo, T é o número de observação da série e $\hat{\sigma}^{2}(\mathrm{k})$ é a variância residual estimada do modelo auto-regressivo de ordem k.

Para a realização do teste de linearidade de Poggi e Portier é necessário fazer a escolha de q pontos, com alta densidade, do suporte da distribuição $h$. Quando esta distribuição for aproximadamente gaussiana os q pontos do suporte, que são da forma $\left(X_{t}, X_{t-1}, \ldots, X_{t-p+1}\right)$, devem ser escolhidos tal que $]-1,25 \hat{\sigma}_{x}{ }^{2}<X_{t}$. ${ }_{\mathrm{i}}<1,25 \hat{\sigma}_{\mathrm{x}}{ }^{2}\left[\right.$, para $\mathrm{i}=0,1, \ldots, \mathrm{p}-1, \mathrm{e} \hat{\sigma}_{\mathrm{x}}{ }^{2}$ é a variância de $\mathrm{X}_{\mathrm{t}}$. Após esta seleção devemos calcular nestes pontos a função distribuição $h$ e devem ser selecionados $q$ 
pontos tais que $\mathrm{D}\left(\mathrm{x}_{\mathrm{i}}, \mathrm{x}_{\mathrm{j}}\right)$, dado por (6.17), é aproximadamente zero, para $\mathrm{x}_{\mathrm{i}} \neq \mathrm{x}_{\mathrm{j}}$. Na prática $\mathrm{q}=3$ ou 4 é suficiente.

Nos testes de normalidade e linearidade de Subba Rao e Gabr (1980) foi utilizada para a estimação do espectro, bi-espectro e bi-coerência a janela espectral de Parzen (ver Tabela 2.1), cuja largura de faixa é $\frac{\sqrt{6}}{M}$. As freqüências principais selecionadas para o teste estão apresentadas nas colunas (3) e (4) da Tabela 5.1. Para as séries TEMP, RESP e TOT foram utilizados os seguintes parâmetros: $K=6$, $\mathrm{d}=10, \mathrm{r}=5$ e $\mathrm{M}=56$ e para a série $\mathrm{PM} 10$ foi utilizado $\mathrm{K}=6, \mathrm{~d}=10, \mathrm{r}=5$ e $\mathrm{M}=48$. Estes parâmetros satisfazem as condições estabelecidas nas equações de (5.20) a (5.23).

\subsection{Total de óbitos por causas respiratórias (RESP)}

\subsubsection{Análise descritiva e transformação}

A série RESP é apresentada na Figura 7.1 e as médias mensais da série são apresentadas na Tabela 7.1; podemos notar que a série apresenta uma não estacionariedade homogênea, uma vez que o total de óbitos por causas respiratórias e os totais médios por causas respiratórias cresceram no inverno, ou seja, entre os meses de junho e agosto e decresceram nos meses subsequentes. Para eliminar esta não estacionariedade foi aplicada à série uma diferença de ordem 1 . A série transformada está apresentada na Figura 7.2. Podemos verificar que a transformação estabilizou a média da série. 


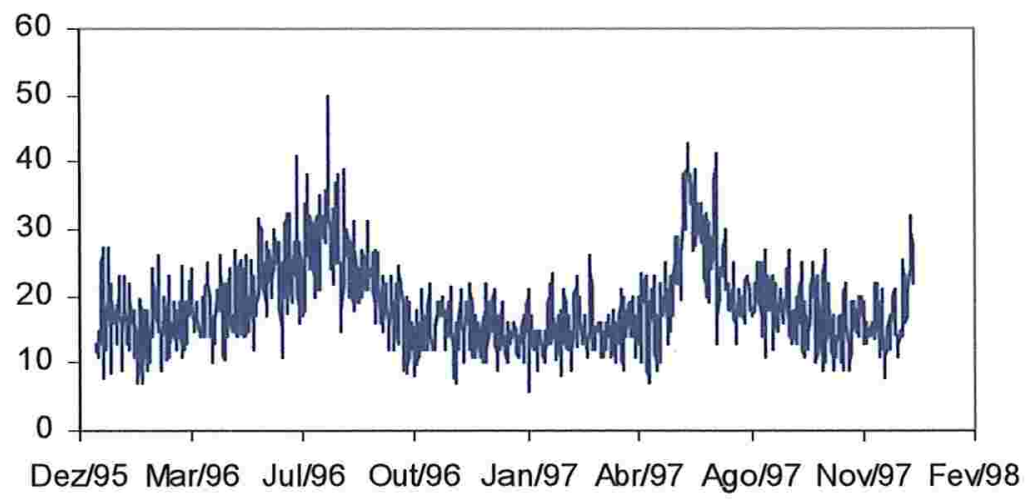

Figura 7.1 - Série RESP.

Tabela 7.1 - Média e desvio padrão mensais calculados a partir dos dados diários da série RESP

\begin{tabular}{lcc|lcc}
\hline Mês & Média & $\begin{array}{c}\text { Desvio } \\
\text { Padrão }\end{array}$ & Mês & Média & $\begin{array}{c}\text { Desvio } \\
\text { Padrão }\end{array}$ \\
\hline Jan/96 & 16,4 & 5,1 & Jan/97 & 13,7 & 2,9 \\
Fev/96 & 15,2 & 4,6 & Fev/97 & 14,8 & 3,8 \\
Mar/96 & 16,9 & 3,9 & Mar/97 & 15,4 & 3,6 \\
Abr/96 & 17,5 & 4,1 & Abr/97 & 15,5 & 3,3 \\
Mai/96 & 20,1 & 4,8 & Mai/97 & 18,1 & 5,0 \\
Jun/96 & 24,7 & 5,6 & Jun/97 & 30,9 & 5,7 \\
Jul/96 & 28,2 & 7,0 & Jul/97 & 21,5 & 5,8 \\
Ago/96 & 25,3 & 5,8 & Ago/97 & 19,4 & 4,2 \\
Set/96 & 20,3 & 4,9 & Set/97 & 17,7 & 4,1 \\
Out/96 & 14,8 & 4,0 & Out/97 & 15,9 & 4,7 \\
Nov/96 & 15,6 & 3,7 & Nov/97 & 16,0 & 3,2 \\
Dez/96 & 15,1 & 3,5 & Dez/97 & 17,3 & 5,5 \\
\hline
\end{tabular}

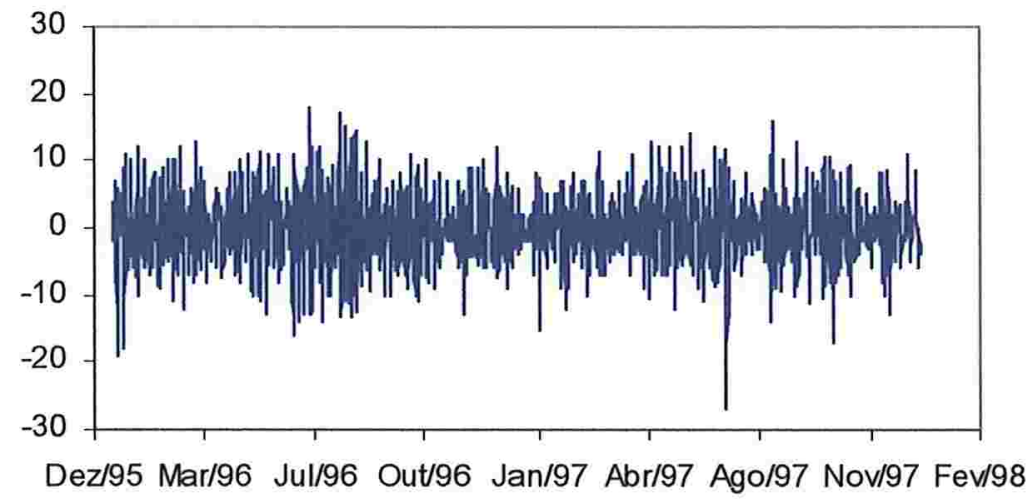

Figura 7.2 - Série (1-B)RESP. 
Podemos notar pela Figura 7.3, em que as 730 observações da série transformada foram divididas em 52 subséries de 14 elementos cada e foram calculadas as médias e os desvios padrões de cada segmento, que a diferença aplicada à série RESP parece ser suficiente.

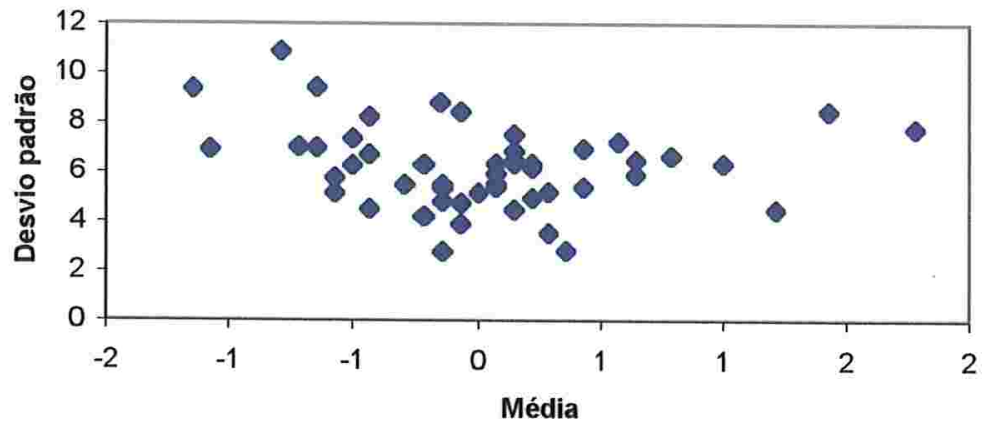

Figura 7.3 - Gráfico do desvio padrão x média de cada uma das 52 subséries da série (1-B)RESP.

Analisando a Figura 7.4, que contém as funções de autocorrelação amostral das séries RESP e (1-B)RESP, notamos que transformação foi adequada e parece ter tornado a série estacionária.
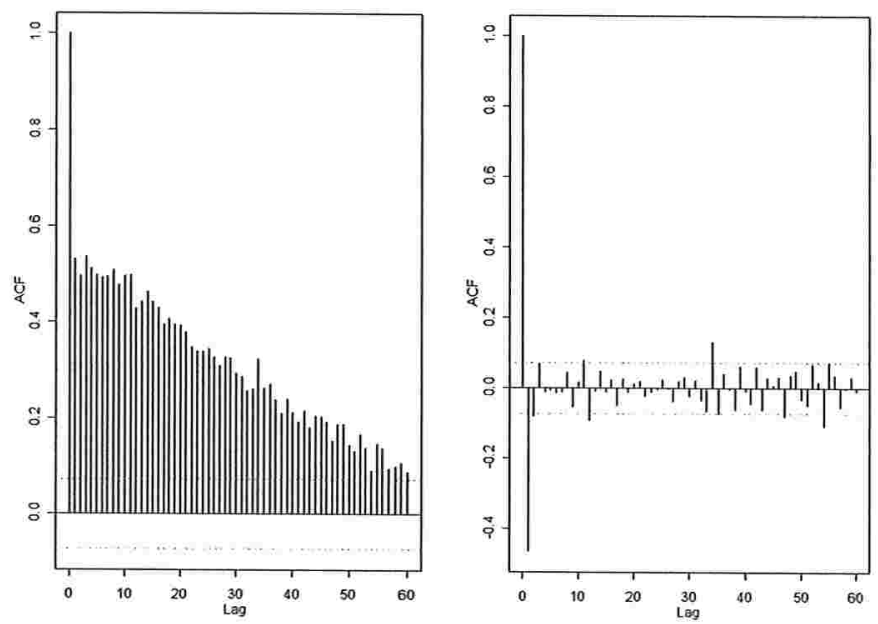

Figura 7.4 - Funções de autocorrelação amostral para as séries RESP e (1-B)RESP. 


\subsubsection{Aplicação dos testes de linearidade e normalidade}

Os testes de linearidade e normalidade descritos nesta seção foram aplicados à diferença de ordem 1 da série total de óbitos por causas respiratórias, que passaremos a denotar por (1-B)RESP.

Para a realização do teste de linearidade do tipo de McLeod e Li, foi ajustado um modelo MA(1) à série com uma diferença, isto é,

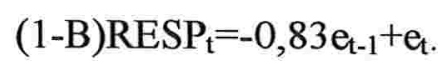

As funções de autocorrelação e autocorrelação parcial amostrais dos resíduos do modelo dado pela expressão (7.1) estão apresentadas na Figura 7.5. O resultado da aplicação do teste, está apresentado na Tabela 7.5.
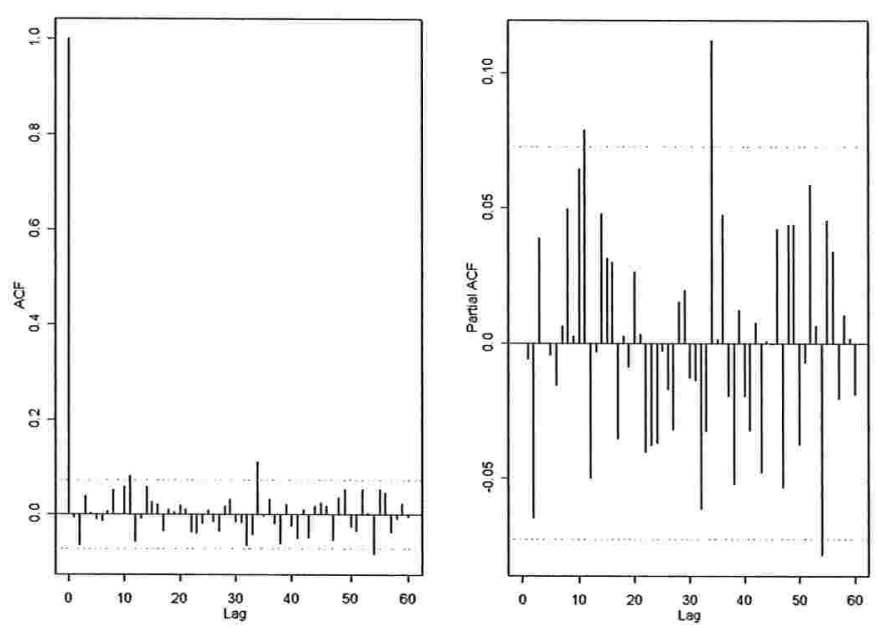

Figura 7.5 - Funções de autocorrelação e autocorrelação parcial amostrais dos resíduos do modelo (7.1).

Com relação ao teste de linearidade de Tsay os valores de AIC e SBC obtidos a partir dos modelos auto-regressivos ajustados estão na Tabela 7.2. 
Notamos que o modelo que se ajustou bem à série (1-B)RESP foi um AR(7), entretanto, esta escolha acarretaria no ajuste de uma regressão multivariada de ordem 28 no terceiro passo do teste, desta forma foi escolhido como um bom modelo o AR(3). O nível descritivo associado ao teste está na Tabela 7.5.

Tabela 7.2 - Valores de AIC e SBC para a série (1-B)RESP

\begin{tabular}{ccc}
\hline AR & AIC & SBC \\
\hline 1 & 4549 & 4554 \\
2 & 4440 & 4449 \\
3 & 4401 & 4414 \\
4 & 4380 & 4398 \\
5 & 4370 & 4393 \\
6 & 4361 & 4388 \\
7 & 4349 & 4381 \\
8 & 4348 & 4385 \\
9 & 4343 & 4384 \\
10 & 4339 & 4385 \\
11 & 4340 & 4390 \\
12 & 4342 & 4397 \\
13 & 4342 & 4401 \\
14 & 4343 & 4408 \\
15 & 4345 & 4414 \\
\hline
\end{tabular}

No teste de linearidade de Poggi e Portier devemos escolher a ordem do modelo auto-regressivo que melhor se ajustou à série e para isto foi utilizado o mesmo critério do teste de Tsay, ou seja, um AR(3). Para a realização do teste é necessário fazer o escolha de q pontos do suporte da distribuição h. Na Figura 7.6 está representado o histograma de (1-B)RESP; como esta distribuição é aproximadamente gaussiana, a função $h$ foi calculada nos pontos $\left(X_{t}, X_{t-1}, X_{t-2}\right)$ tal que ]-7,69< $<X_{t-i}<7,69$ [ para $i=0,1,2$. A Tabela 7.3 apresenta os pontos que foram selecionados para o teste e o tempo em que a observação ocorreu ( $\left.\mathrm{T}^{*}\right)$. Estes pontos, que possuem alta densidade, foram selecionados utilizando o histograma da função h que está apresentado na Figura7.7. A Tabela 7.4 apresenta o valor da 
função h para cada ponto selecionado e a distância entre estes pontos; como esta distância é aproximadamente zero, os pontos foram selecionados adequadamente. O resultado da aplicação do teste, está apresentado na Tabela 7.5.

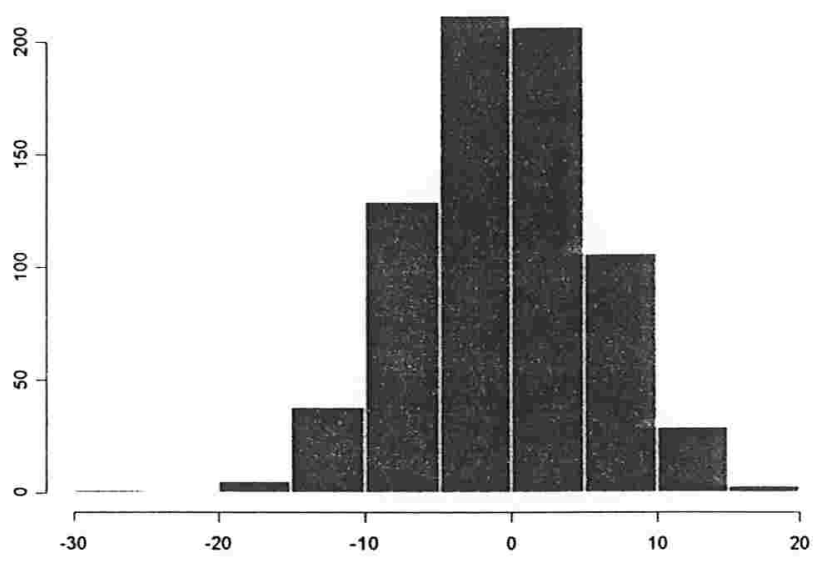

Figura 7.6 - Histograma de (1-B)RESP.

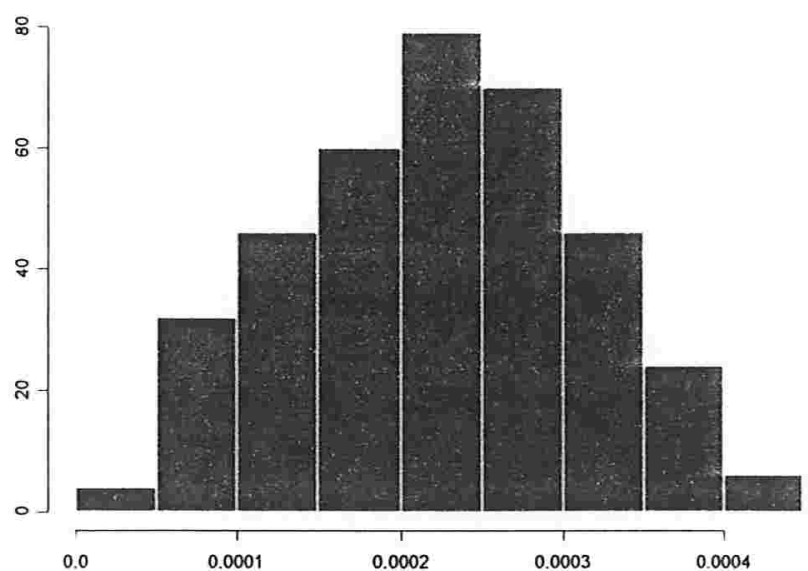

Figura 7.7 - Histograma da função h para (1B)RESP. 
Tabela 7.3 - Pontos selecionados para o teste de Poggi e Portier

\begin{tabular}{cccc}
\hline $\mathrm{T}^{*}$ & $\mathrm{X}_{\mathbf{t}}$ & $\mathrm{X}_{\mathrm{t}-1}$ & $\mathrm{X}_{\mathbf{t}-\mathbf{2}}$ \\
\hline 460 & -4 & -4 & 7 \\
422 & -6 & 0 & -2 \\
67 & 3 & -1 & -1 \\
410 & 1 & 2 & 3 \\
\hline
\end{tabular}

Tabela 7.4 - Função $h$ e distância entre os pontos selecionados (D)

\begin{tabular}{cc|cc}
\hline $\mathrm{T}^{*}$ & $\mathrm{~h}$ & Pontos & $\mathrm{D}$ \\
\hline 460 & 0,00016 & $(460,422)$ & $1,50 \times 10^{-11}$ \\
422 & 0,00020 & $(460,067)$ & $1,28 \times 10^{-11}$ \\
067 & 0,00024 & $(460,410)$ & $2,25 \times 10^{-11}$ \\
410 & 0,00028 & $(422,067)$ & $1,85 \times 10^{-11}$ \\
& & $(422,410)$ & $3,12 \times 10^{-11}$ \\
& & $(067,410)$ & $1,46 \times 10^{-11}$ \\
\hline
\end{tabular}

Os testes de linearidade e normalidade de Subba Rao e Gabr foram aplicados nas 7 freqüências principais dadas pelas colunas (3) e (4) da Tabela 5.1, sendo que em torno de cada frequências principal foi selecionada uma amostra de 21 frequeências satisfazendo (5.8) e (5.9) com $\mathrm{r}=5$. Os resultados das aplicações dos testes estão nas Tabelas 7.5 e 7.6.

Nos testes de normalidade e linearidade de Brillinger e Irizarry em que a série foi particionada em 46 subséries de tamanho 16 obtivemos 29 freqüências em que o espectro e o bi-espectro foram calculados. Os níveis descritivos associados aos testes estão nas Tabelas 7.5 e 7.6. Nas Figuras 7.8 e 7.9 estão apresentados os níveis descritivos associados aos testes de linearidade e normalidade. Com relação ao teste de linearidade, $31,03 \%$ dos niveis descritivos associados ao teste são menores do que 0,05 e $89,65 \%$ dos níveis descritivos, associados ao teste de normalidade, são menores do que 0,05 . 


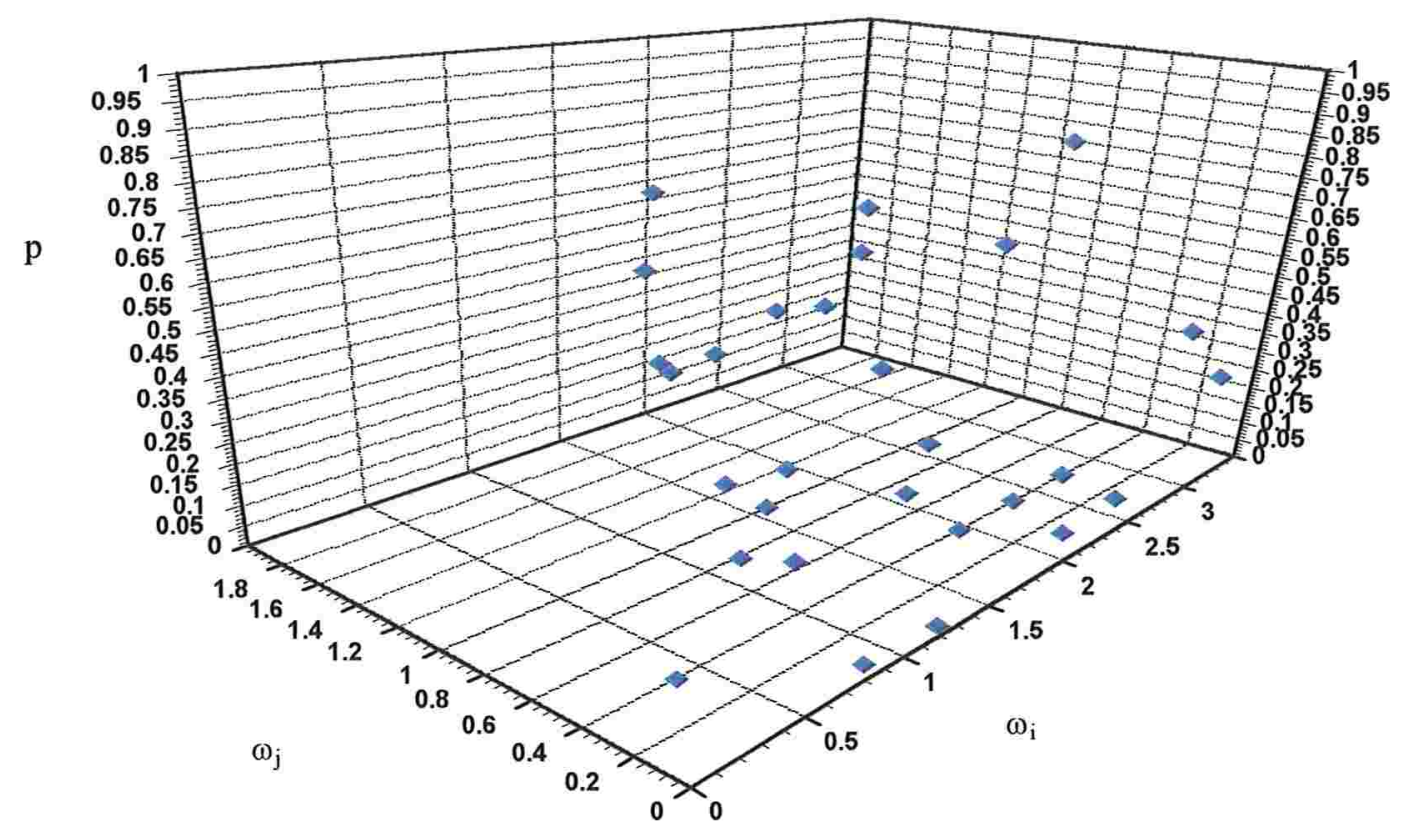

Figura 7.8 - Níveis descritivos associados ao teste de linearidade.

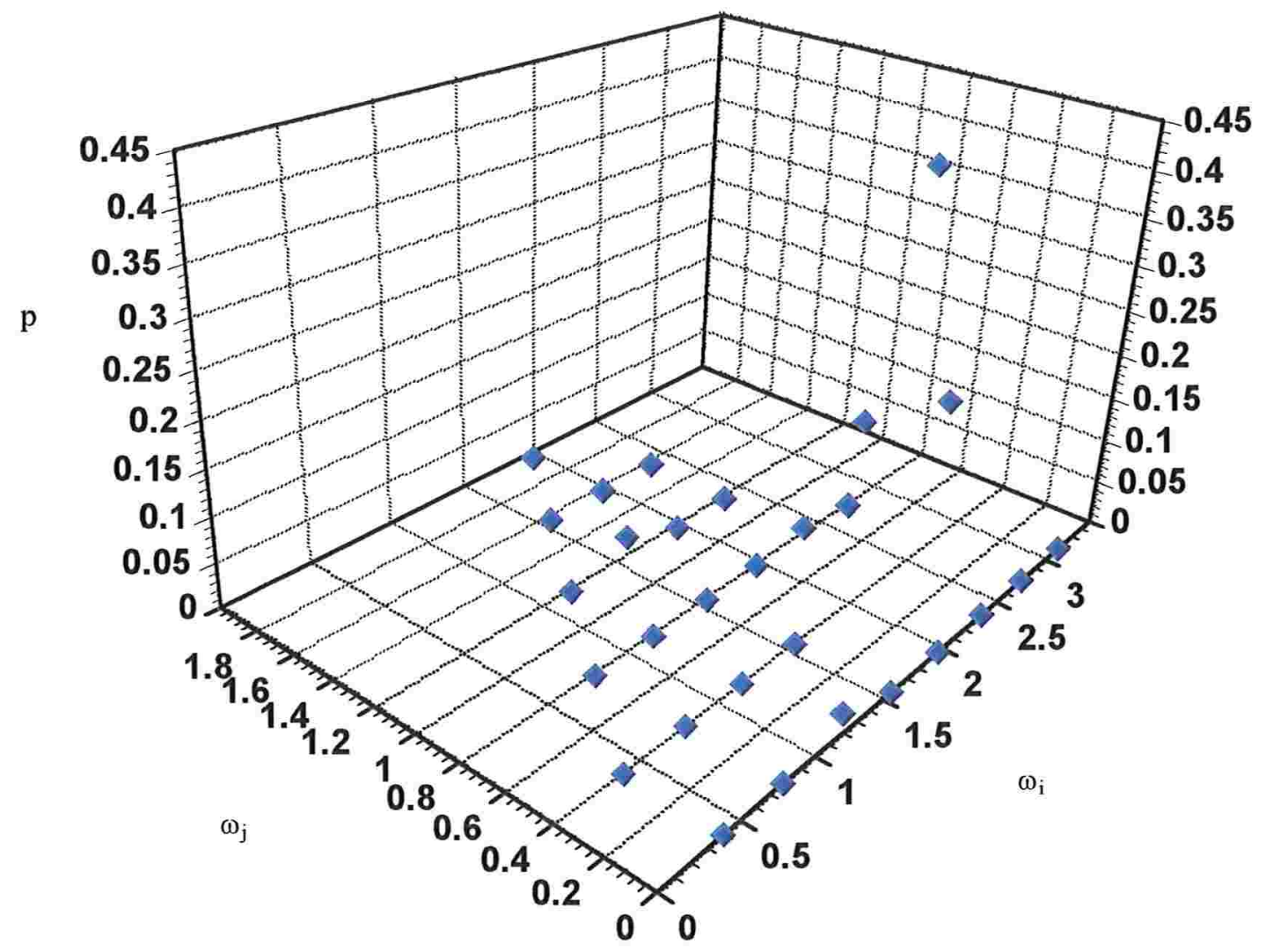

Figura 7.9 - Níveis descritivos associados ao teste de normalidade. 


\section{Conclusão}

Os testes de linearidade e normalidade descritos nesta seção, cujos resultados estão nas Tabela 7.5 e 7.6, indicam que a série (1-B)RESP não possui um comportamento linear nem gaussiano.

Tabela 7.5 - Testes de linearidade para a série (1-B)RESP

\begin{tabular}{l|l|c|c|c|c}
\hline & Teste & Estatística & Estimativa & $\mathrm{p}$ & $\begin{array}{c}\text { percentual } \\
\mathrm{de} \mathrm{p}<0,05\end{array}$ \\
\hline & $\begin{array}{l}\mathrm{McLeod} \mathrm{e} \\
\mathrm{Li} \\
\text { Domínio do } \\
\text { tempo }\end{array}$ & $\mathrm{Q}$ & 112,72 & 0,0000 & - \\
& $\begin{array}{l}\text { Poggi e } \\
\text { Portier }\end{array}$ & $\mathrm{T}_{\mathrm{q}}(\mathrm{n})$ & 1097,13 & 0,000 & - \\
\hline $\begin{array}{l}\text { Domínio da } \\
\text { freqüência }\end{array}$ & $\begin{array}{l}\text { Subba Rao e } \\
\text { Gabr } \\
\text { Brillinger e } \\
\text { Irizarry }\end{array}$ & $\mathrm{X}^{*}\left(\omega_{\mathrm{i}}, \omega_{\mathrm{j}}\right)$ & - & - & 31,03 \\
\hline
\end{tabular}

p: nível descritivo associado ao teste.

Tabela 7.6 - Testes de normalidade no domínio da freqüência para a série (1-B) RESP

\begin{tabular}{l|c|c|c|c}
\hline Teste & Estatística & Estimativa & $\mathrm{p}$ & $\begin{array}{c}\text { percentual } \\
\text { de } \mathrm{p}<0,05\end{array}$ \\
\hline Subba Rao e Gabr & $\Im_{1}$ & 80,55 & 0,0000 & - \\
Brillinger e Irizarry & $\hat{\gamma}_{\mathrm{B}}{ }^{2}\left(\omega_{1}, \omega_{2}\right)$ & - & - & 89,65 \\
\hline
\end{tabular}

p: nível descritivo associado ao teste. 


\subsection{Total de óbitos para crianças de 1 a 11 meses (TOT)}

\subsubsection{Análise descritiva e transformação}

A série TOT é apresentada na Figura 7.10 e as médias mensais desta série são apresentadas na Tabela 7.7. Podemos notar um comportamento não estacionário homogêneo da série, uma vez que o total de óbitos e os valores médios mensais, cresceram entre junho e agosto e decresceram nos meses subsequentes. Para eliminar esta não estacionariedade foi aplicada à série uma diferença de ordem 1. A série transformada está apresentada na Figura 7.11. Podemos verificar que a transformação estabilizou a média da série.

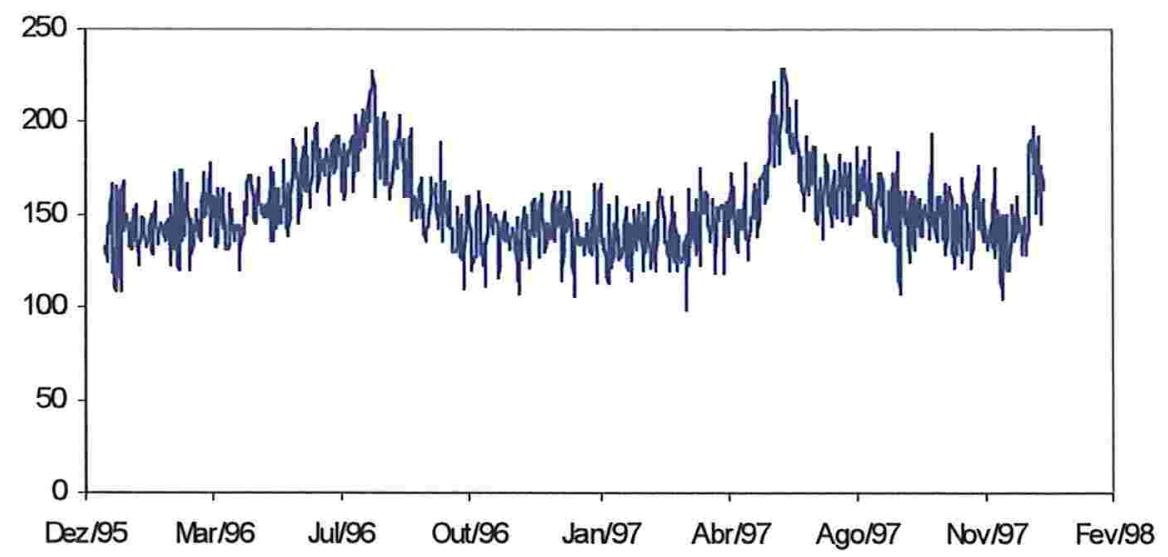

Figura 7.10 - Série TOT. 
Tabela 7.7 - Média e desvio padrão mensais calculados a partir dos dados diários da série TOT

\begin{tabular}{lcclcc}
\hline Mês & Média & $\begin{array}{c}\text { Desvio } \\
\text { Padrão }\end{array}$ & Mês & Média & $\begin{array}{c}\text { Desvio } \\
\text { Padrão }\end{array}$ \\
\hline Jan/96 & 140,2 & 15,9 & Jan/97 & 137,1 & 13,6 \\
Fev/96 & 142,0 & 12,2 & Fev/97 & 138,0 & 12,1 \\
Mar/96 & 147,8 & 15,0 & Mar/97 & 137,0 & 15,1 \\
Abr/96 & 148,5 & 13,1 & Abr/97 & 147,2 & 13,3 \\
Mai/96 & 157,9 & 15,0 & Mai/97 & 152,5 & 13,7 \\
Jun/96 & 177,4 & 11,6 & Jun/97 & 193,0 & 18,3 \\
Jul/96 & 188,3 & 16,5 & Jul/97 & 162,7 & 13,7 \\
Ago/96 & 176,7 & 16,1 & Ago/97 & 160,3 & 13,3 \\
Set/96 & 152,1 & 13,5 & Set/97 & 148,4 & 14,7 \\
Out/96 & 139,8 & 14,4 & Out/97 & 148,8 & 14,3 \\
Nov/96 & 137,5 & 11,6 & Nov/97 & 143,6 & 15,4 \\
Dez/96 & 143,3 & 13,3 & Dez/97 & 152,1 & 21,2 \\
\hline
\end{tabular}

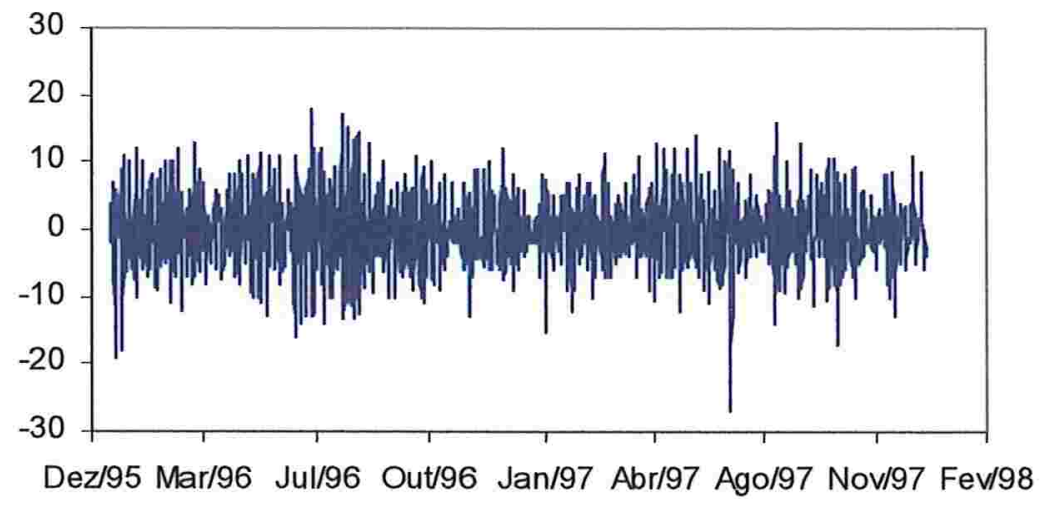

Figura 7.11 - Série (1-B)TOT.

Podemos notar pela Figura 7.12, em que as 730 observações da série transformada foram divididas em 52 subséries de 14 elementos cada e foram calculadas as médias e os desvios padrões de cada segmento, que a diferença aplicada à série TOT parece ser suficiente. 


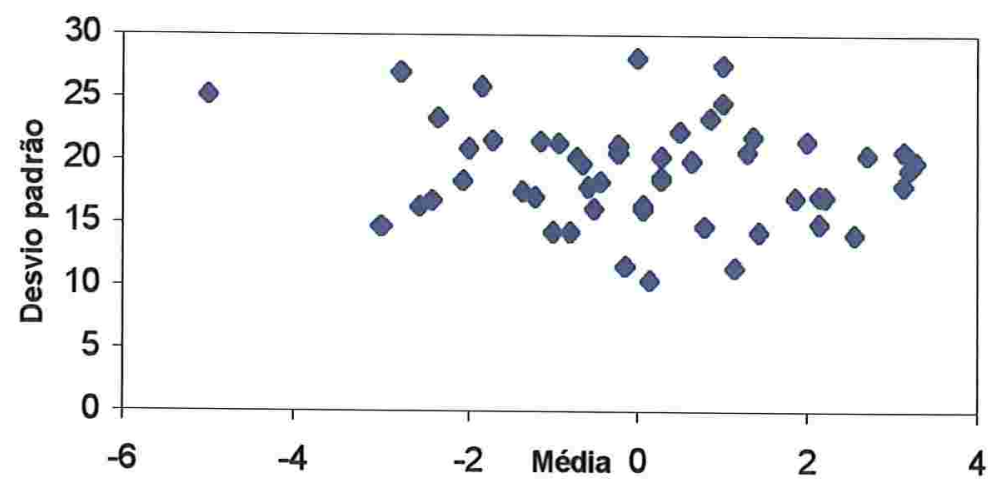

Figura 7.12 - Gráfico do desvio padrão x média de cada uma das 52 subséries da série (1-B)TOT.

Analisando a Figura 7.13, em que estão representadas as funções de autocorrelação amostral das séries TOT e (1-B)TOT, notamos que a transformação foi adequada e parece ter tornado a série estacionária.
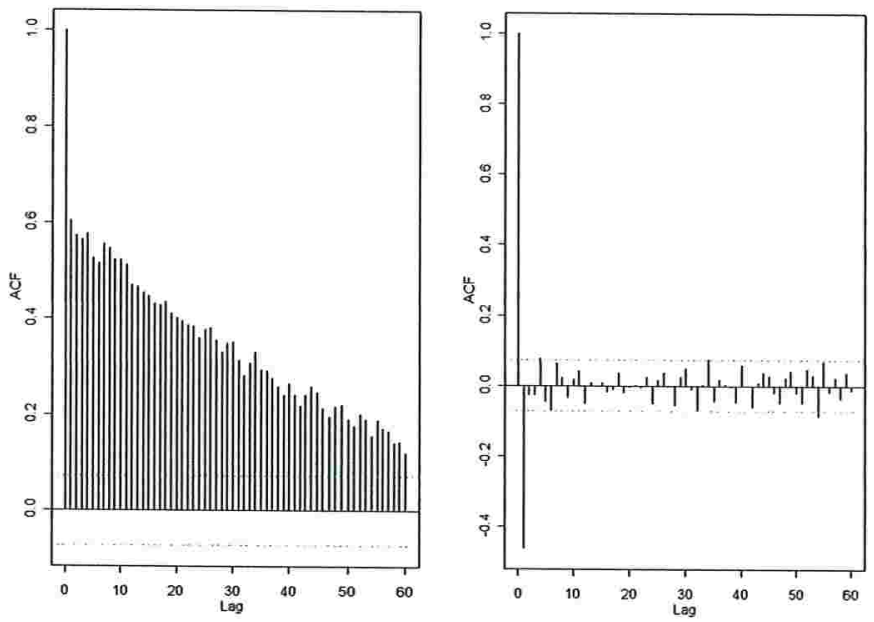

Figura 7.13 - Funções de autocorrelação amostral para as séries TOT e (1-B)TOT. 


\subsubsection{Aplicação dos testes de linearidade e normalidade}

Os testes de linearidade e normalidade descritos nesta seção foram aplicados à diferença de ordem 1 da série total de óbitos para crianças de 1 a 11 meses, que passaremos a denotar por (1-B)TOT.

Para a realização do teste de linearidade de McLeod e Li, foi ajustado um modelo MA(1) à série com uma diferença, isto é,

$$
(1-\mathrm{B}) \mathrm{TOT}_{\mathrm{t}}=-0,80 \mathrm{e}_{\mathrm{t}-1}+\mathrm{e}_{\mathrm{t}}
$$

As funções de autocorrelação e autocorrelação parcial amostrais dos resíduos do modelo dado pela expressão (7.2) estão apresentadas na Figura 7.14. Na Tabela 7.11 está apresentado o resultado da aplicação do teste.
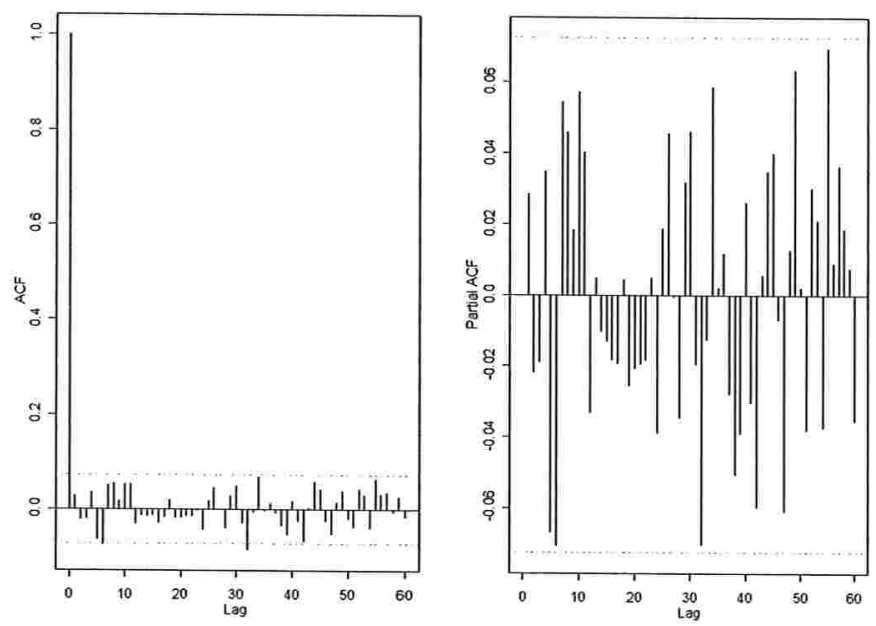

Figura 7.14 - Funções de autocorrelação e autocorrelação parcial amostrais dos resíduos do modelo (7.2).

Com relação ao teste de linearidade de Tsay os valores de AIC e SBC, obtidos a partir dos modelos auto-regressivos ajustados, estão na Tabela 7.8. 
Notamos que o modelo que se ajustou bem à série (1-B)TOT foi um AR(7), entretanto, esta escolha acarretaria no ajuste de uma regressão multivariada de ordem 28 no terceiro passo do teste, desta forma foi escolhido como um bom modelo o AR(3). O nível descritivo associado ao teste está na Tabela 7.11.

\begin{tabular}{ccc} 
Tabela & \multicolumn{3}{c}{ 7.8 - Valores de AIC e } \\
SBC para a série (1-B) TOT \\
\hline 1 & 6189 & 6194 \\
2 & 6120 & 6129 \\
3 & $\mathbf{6 0 7 2}$ & 6085 \\
4 & 6065 & 6083 \\
5 & 6060 & 6083 \\
6 & 6036 & 6064 \\
7 & 6026 & 6059 \\
8 & 6025 & 6062 \\
9 & 6022 & 6063 \\
10 & 6022 & 6068 \\
11 & 6023 & 6074 \\
12 & 6025 & 6080 \\
13 & 6027 & 6087 \\
14 & 6029 & 6094 \\
15 & 6031 & 6100 \\
\hline
\end{tabular}

Para a realização do teste de linearidade de Poggi e Portier devemos escolher a ordem do modelo auto-regressivo que melhor se ajustou à série e para isto foi utilizado o mesmo critério do teste de Tsay, ou seja, um AR(3). Para a realização do teste é necessário fazer o escolha de q pontos do suporte da distribuição h. O histograma de (1-B)TOT está representado na Figura 7.15; como esta distribuição é aproximadamente gaussiana, a função $h$ foi calculada nos pontos $\left(X_{t}, X_{t-1}, X_{t-2}\right)$ tal que $]-23,0<X_{t-i}<23,0[$, para $i=0,1$, 2. A Tabela 7.9 apresenta os pontos selecionados para o teste e o tempo em que a observação ocorreu $\left(\mathrm{T}^{*}\right)$. Estes pontos, que possuem alta densidade, foram selecionados pelo histograma da função $h$ que está apresentado na Figura 7.16. A Tabela 7.10 apresenta o valor da função $h$ para cada ponto selecionado e a distância entre estes 
pontos. Como esta distância é aproximadamente zero, os pontos foram selecionados adequadamente. O resultado da aplicação do teste, está apresentado na Tabela 7.11.

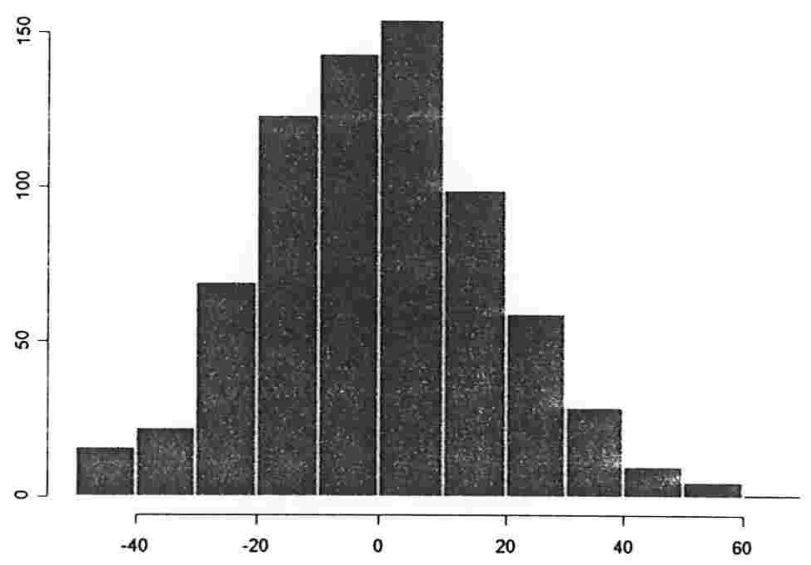

Figura 7.15 - Histograma de (1-B)TOT.

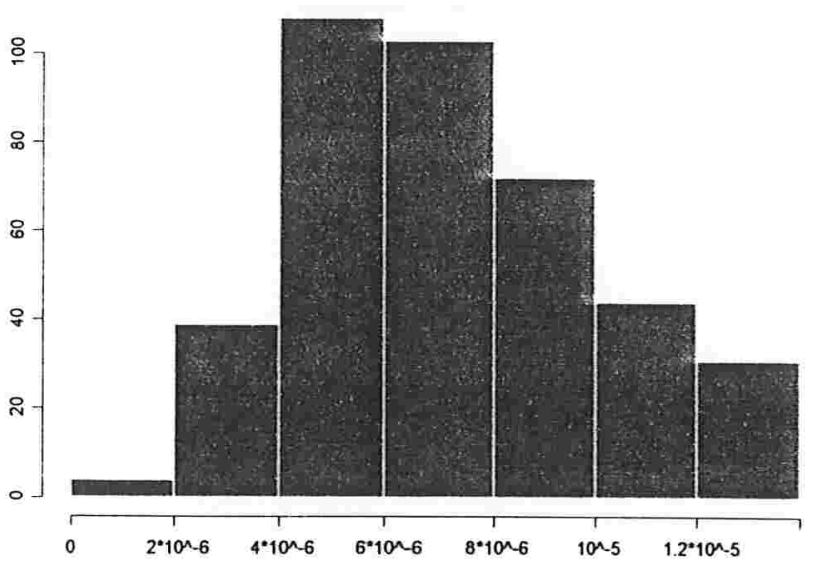

Figura 7.16 - Histograma da função h para (1B)TOT. 
Tabela 7.9 - Pontos selecionados para o teste de Poggi e Portier

\begin{tabular}{cccc}
\hline $\mathrm{T}^{*}$ & $\mathrm{X}_{\mathrm{t}}$ & $\mathrm{X}_{\mathrm{t}-1}$ & $\mathrm{X}_{\mathrm{t}-2}$ \\
\hline 080 & 16 & 12 & -11 \\
028 & -2 & 8 & 14 \\
223 & 10 & 13 & -9 \\
635 & 7 & -3 & -17 \\
\hline
\end{tabular}

Tabela 7.10 - Função $h$ e distância entre os pontos selecionados (D)

\begin{tabular}{cccc}
\hline T* $^{*}$ & $\mathrm{~h}$ & Pares & $\mathrm{D}$ \\
\hline 080 & $4,3 \times 10^{-6}$ & $(080,028)$ & $3,6 \times 10^{-14}$ \\
028 & $5,1 \times 10^{-6}$ & $(080,223)$ & $8,6 \times 10^{-13}$ \\
223 & $6,0 \times 10^{-6}$ & $(080,635)$ & $1,2 \times 10^{-13}$ \\
635 & $8,0 \times 10^{-6}$ & $(028,223)$ & $6,6 \times 10^{-14}$ \\
& & $(028,635)$ & $2,5 \times 10^{-14}$ \\
& & $(223,635)$ & $1,6 \times 10^{-13}$ \\
\hline
\end{tabular}

Os testes de linearidade e normalidade de Subba Rao e Gabr foram aplicados nas 7 freqüências principais dadas pelas colunas (3) e (4) da Tabela 5.1, sendo que em torno de cada freqüências principal foi selecionada uma amostra de 21 freqüências satisfazendo (5.8) e (5.9) com $r=5$. Os resultados das aplicações dos testes estão nas Tabelas 7.11 e 7.12 .

Nos testes de normalidade e linearidade de Brillinger e Irizarry a série foi particionada em 46 subséries de tamanho 16, desta forma, obtivemos 29 freqüências em que o espectro e o bi-espectro foram calculados. Os niveis descritivos associados aos testes estão nas Tabelas 7.11 e 7.12. Os níveis descritivos associados aos testes de linearidade e normalidade estão apresentados nas Figuras 7.17 e 7.18. Com relação ao teste de linearidade, 37,93 \% dos niveis descritivos são menores do que 0,05 e $62,08 \%$ dos niveis descritivos, associados ao teste de normalidade, são menores do que 0,05 . 


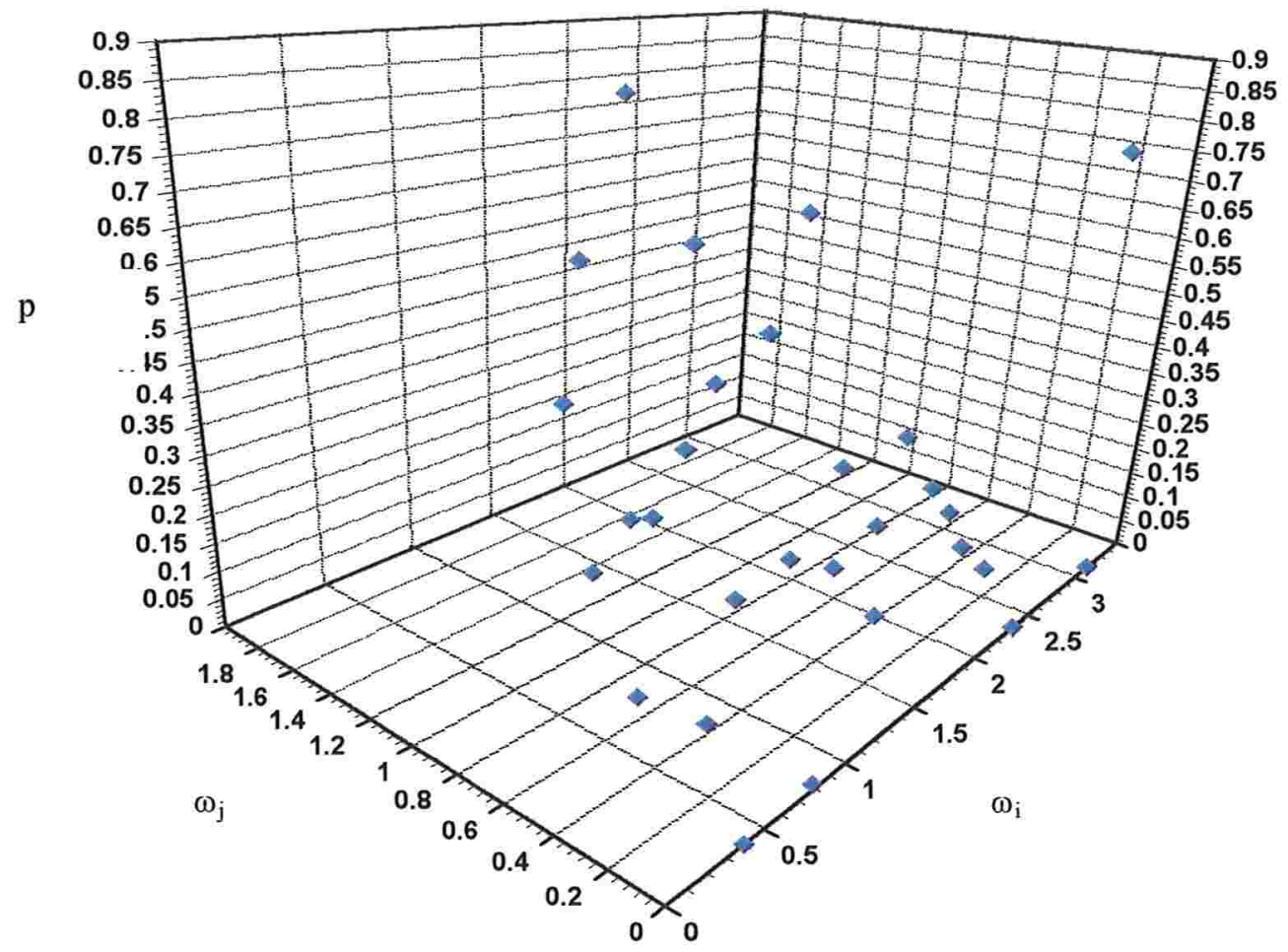

Figura 7.17 - Níveis descritivos associados ao teste de linearidade.

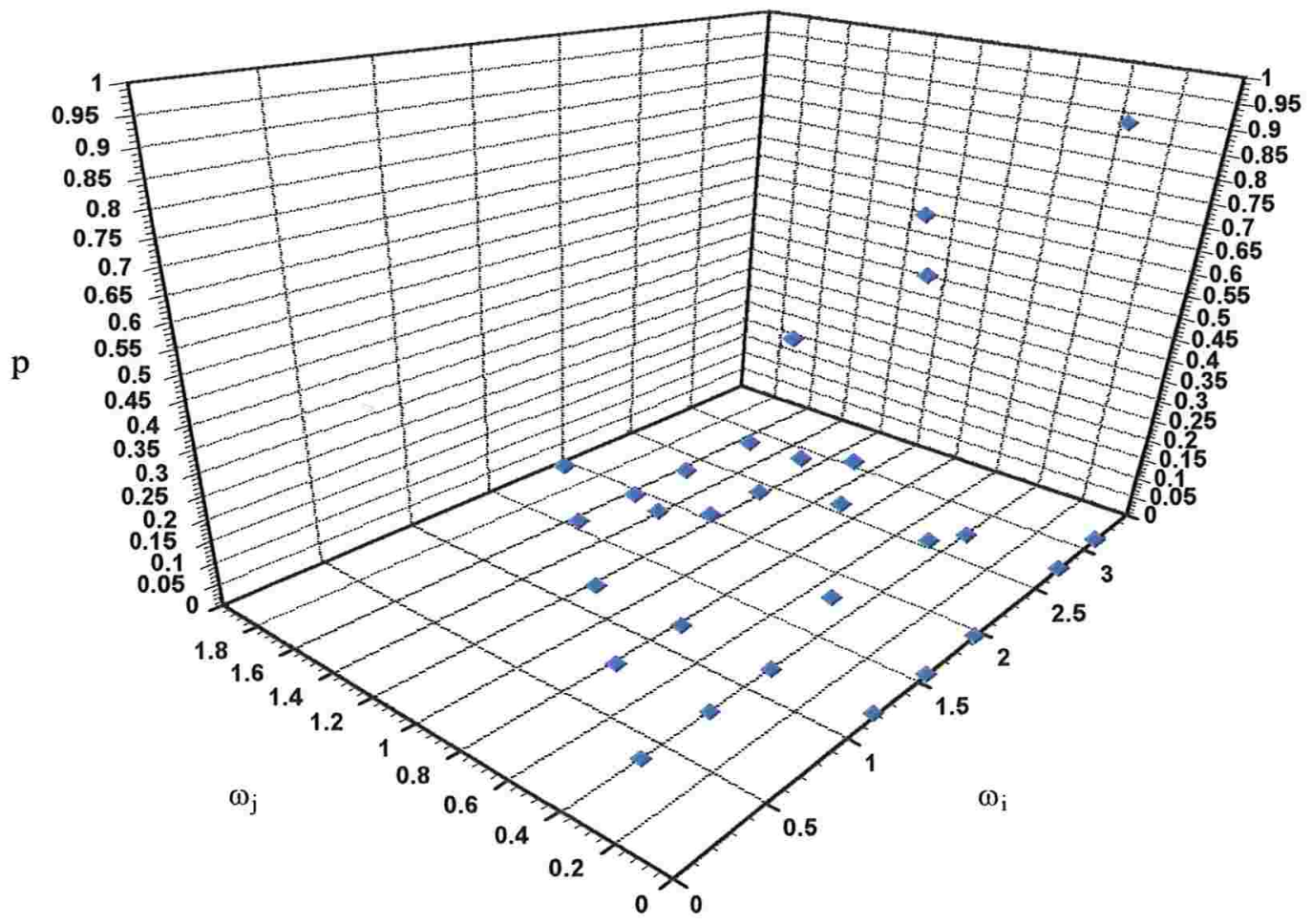

Figura 7.18 - Níveis descritivos associados ao teste de normalidade. 


\section{Conclusão}

Os resultados dos testes de linearidade e normalidade realizados na série (1-B)TOT, estão nas Tabelas 7.11 e 7.12. Notamos que a série não apresenta um comportamento linear nem gaussiano. Os testes de linearidade de McLeod e Li e de Tsay indicam que a série possui um comportamento linear.

Tabela 7.11 - Testes de linearidade para a série (1-B)TOT

\begin{tabular}{l|l|c|c|c|c}
\hline & Teste & Estatística & Estimativa & $\mathrm{p}$ & $\begin{array}{c}\text { percentual } \\
\mathrm{de} \mathrm{p}<0,05\end{array}$ \\
\hline & $\begin{array}{l}\text { McLeod e } \\
\mathrm{Li}\end{array}$ & $\mathrm{Q}$ & 60,10 & 0,4718 & - \\
$\begin{array}{l}\text { Domínio do } \\
\text { tempo }\end{array}$ & Tsay & $\mathrm{F}$ & 1,53 & 0,1650 & - \\
\hline $\begin{array}{l}\text { Poggi e } \\
\text { Portier }\end{array}$ & $\mathrm{T}_{\mathrm{q}}(\mathrm{n})$ & 43093,0 & 0,0000 & - \\
$\begin{array}{l}\text { Domínio da } \\
\text { freqüência }\end{array}$ & $\begin{array}{l}\text { Subba Rao e } \\
\text { Gabr } \\
\text { Brillinger e } \\
\text { Irizarry }\end{array}$ & $\mathrm{I}^{*}\left(\omega_{\mathrm{i}}, \omega_{\mathrm{j}}\right)$ & - & - & 37,93 \\
\hline
\end{tabular}

p: nível descritivo associado ao teste

Tabela 7.12 - Testes de normalidade no domínio da freqüência para a série (1B)TOT

\begin{tabular}{l|c|c|c|c}
\hline Teste & Estatística & Estimativa & $\mathrm{p}$ & $\% \mathrm{p}<0,05$ \\
\hline Subba Rao e Gabr & $\Im_{1}$ & 16,47 & 0,0000 & - \\
Brillinger e Irizarry & $\hat{\gamma}_{\mathrm{B}}{ }^{2}\left(\omega_{1}, \omega_{2}\right)$ & - & - & 62,08 \\
\hline
\end{tabular}

p: nível descritivo associado ao teste 
7.4 Média da concentração do agente poluidor PM10 (PM10)

\subsubsection{Análise descritiva e transformação}

A série PM10 é apresentada na Figura 7.19; podemos perceber que a média e a variância da série não são constantes em relação ao tempo indicando uma não estacionariedade da série. Pela Tabela 7.13, dos dados mensais do agente poluidor PM10, notamos que entre janeiro e abril de 96 e entre março e maio de 97 ocorreram os maiores valores médios e desvios padrões da série.

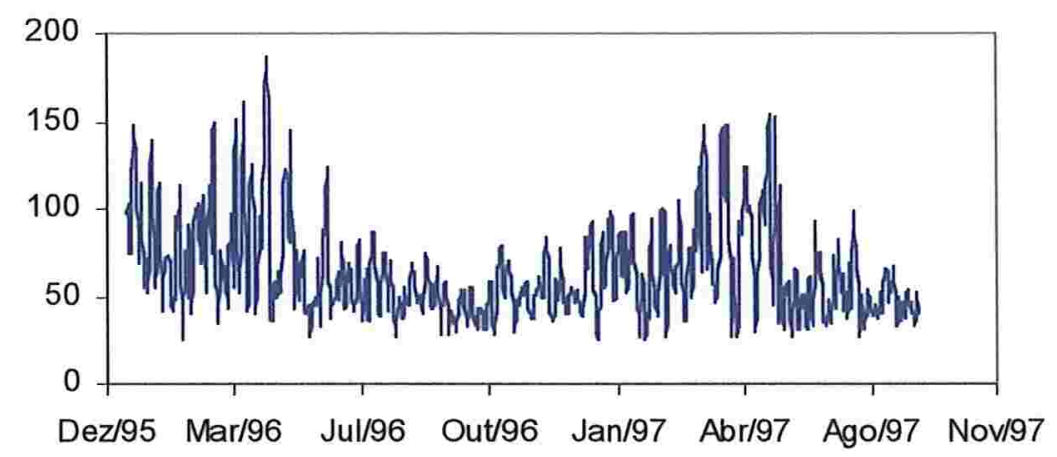

Figura 7.19 - Série PM10. 
Tabela 7.13 - Média e desvio padrão mensais calculados a partir dos dados diários da série PM10

\begin{tabular}{lcc|lcc}
\hline Mês & Média & $\begin{array}{c}\text { Desvio } \\
\text { Padrão }\end{array}$ & Mês & Média & $\begin{array}{l}\text { Desvio } \\
\text { Padrão }\end{array}$ \\
\hline Jan/96 & 89,8 & 29,5 & Jan/97 & 67,5 & 18,8 \\
Fev/96 & 73,7 & 23,4 & Fev/97 & 57,5 & 23,5 \\
Mar/96 & 79,7 & 30,5 & Mar/97 & 78,5 & 28,4 \\
Abr/96 & 90,5 & 47,1 & Abr/97 & 80,0 & 36,8 \\
Mai/96 & 68,7 & 31,2 & Mai/97 & 85,1 & 35,0 \\
Jun/96 & 58,7 & 19,3 & Jun/97 & 52,0 & 15,8 \\
Jul/96 & 58,3 & 16,5 & Jul/97 & 54,5 & 18,5 \\
Ago/96 & 51,6 & 10,7 & Ago/97 & 47,5 & 9,8 \\
Set/96 & 44,7 & 9,8 & Set/97 & 43,9 & 6,6 \\
Out/96 & 48,8 & 14,2 & & & \\
Nov/96 & 51,5 & 11,8 & & & \\
Dez/96 & 57,0 & 15,2 & & & \\
\hline
\end{tabular}

A necessidade de uma transformação na série pode ser percebida pela Figura 7.20, em que as 623 observações da série foram divididas em 44 subséries de 14 elementos cada e foram calculadas as médias e os desvios padrões de cada segmento. Com base nesta figura, podemos verificar o aumento da variância dos dados com o aumento da média das subséries. Sendo assim, aplicamos o logarítmo nos valores da série PM10. A série transformada está apresentada na Figura 7.21, podemos notar que a transformação parece estabilizar a variabilidade da série.

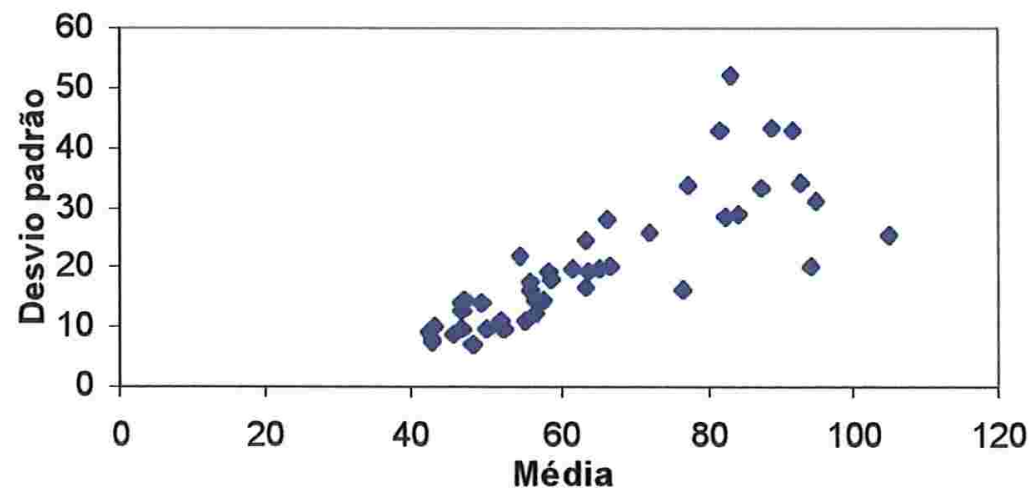

Figura 7.20 - Gráfico do desvio padrão x média de cada uma das 44 subséries da série PM10. 


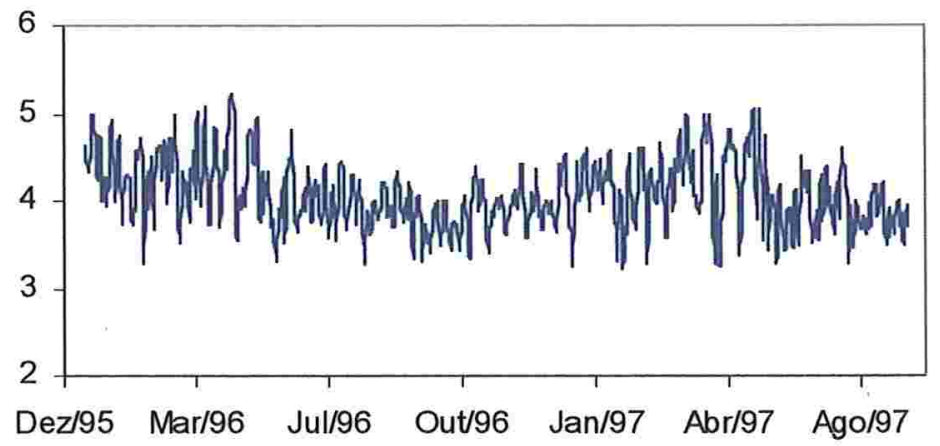

Figura 7.21 - Logarítmo da série PM10.

Analisando a Figura 7.22, que contém as funções de autocorrelação amostral da série PM10 e do logarítmo da série PM10, verificamos que as duas funções de autocorrelação amostral apresentam um comportamento semelhante e parecem indicar uma periodicidade na série.
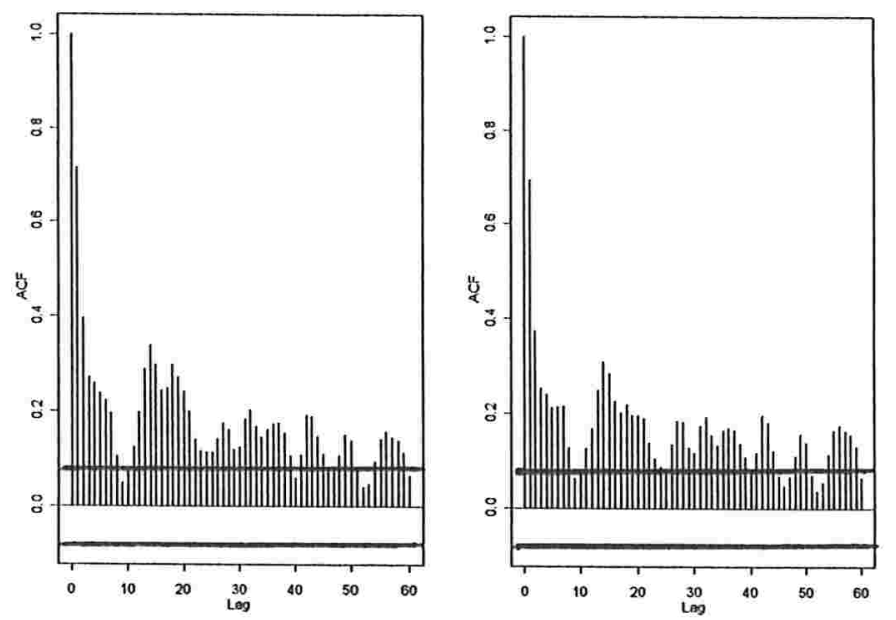

Figura 7.22 - Funções de autocorrelação amostral para as séries PM10 e LPM10. 


\subsubsection{Aplicação dos testes de linearidade e normalidade}

Os testes de linearidade e normalidade descritos nesta seção foram aplicados ao logarítmo da série média da concentração do agente poluidor PM10, que passaremos a denotar por LPM10.

Para a realização do teste de linearidade de McLeod e Li, foi ajustado um modelo auto-regressivo à série, isto é,

LPM10 $-4=0,86 \mathrm{LPM}_{\mathrm{t}} \mathrm{t}_{\mathrm{t}-1}-0,35 \mathrm{LPM}_{10}+2+0,17 \mathrm{LPM10}_{\mathrm{t}-3}+0,08 \mathrm{LPM}_{\mathrm{t}} \mathrm{t}_{\mathrm{t}-12}+\mathrm{e}$.

As funções de autocorrelação e autocorrelação parcial amostrais do resíduo do modelo dado pela expressão (7.3) estão apresentadas na Figura 7.23. O resultado da aplicação do teste, está apresentado na Tabela 7.17.
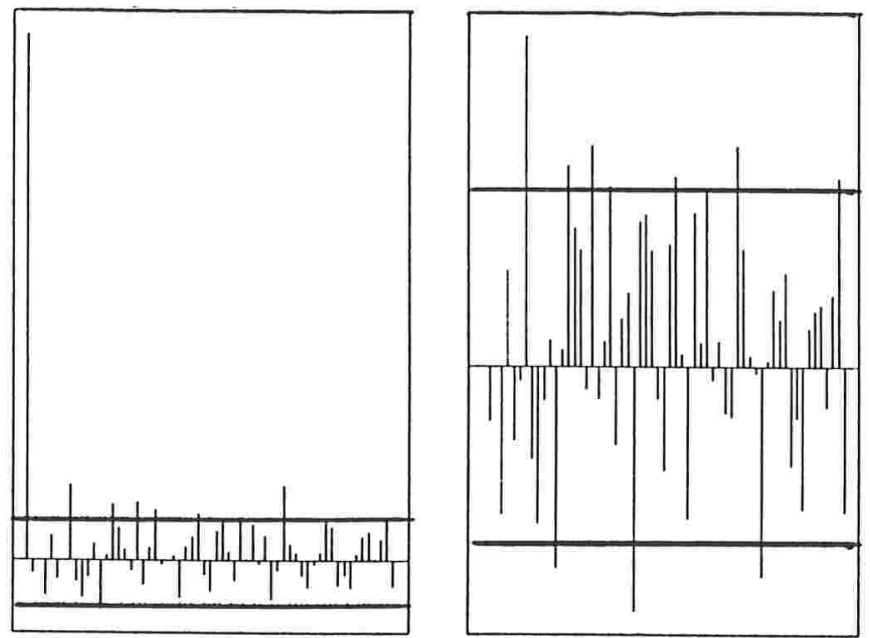

Figura 7.23 - Funções de autocorrelação e autocorrelação parcial amostrais dos resíduos do modelo (7.3). 
Com relação ao teste de linearidade de Tsay os valores de AIC e SBC obtidos a partir dos modelos auto-regressivos ajustados estão na Tabela 7.14, notamos que o modelo que se ajustou bem à série LPM10 foi um $\operatorname{AR}(13)$, entretanto, esta escolha acarretaria no ajuste de uma regressão multivariada de ordem 91 no terceiro passo do teste, desta forma foi escolhido como um bom modelo o $\mathrm{AR}(3)$. O nível descritivo associado ao teste está na Tabela 7.17.

Tabela 7.14 - Valores de AIC e SBC para a série LPM10

\begin{tabular}{ccc}
\hline AR & AIC & SBC \\
\hline 1 & 303 & 308 \\
2 & 305 & 314 \\
$\mathbf{3}$ & $\mathbf{2 3 4}$ & $\mathbf{2 4 8}$ \\
4 & 217 & 235 \\
5 & 213 & 236 \\
6 & 192 & 219 \\
7 & 189 & 220 \\
8 & 189 & 224 \\
9 & 184 & 224 \\
10 & 178 & 223 \\
11 & 175 & 224 \\
12 & 166 & 219 \\
13 & 145 & 202 \\
14 & 141 & 203 \\
15 & 141 & 207 \\
\hline
\end{tabular}

No teste de linearidade de Poggi e Portier devemos escolher a ordem do modelo auto-regressivo que melhor se ajustou à série e para isto foi utilizado o mesmo critério do teste de Tsay, ou seja, um AR(3). Para a realização do teste é necessário fazer o escolha de q pontos do suporte da distribuição h. Na Figura 7.24 está representado o histograma de LPM10. A função $h$ foi calculada nos pontos $\left(X_{t}, X_{t-1}, X_{t-2}\right)$ tal que $] 3,75<X_{t-1}<4,25[$, para $i=0,1,2$. A Tabela 7.15 apresenta os pontos que foram selecionados para o teste e o tempo em que a observação ocorreu $\left(\mathrm{T}^{*}\right)$. Estes pontos, que possuem alta densidade, foram selecionados utilizando o 
histograma da função h que está apresentado na Figura 7.25. A Tabela 7.16 apresenta o valor da função h para cada ponto selecionado e a distância entre estes pontos; como esta distância é aproximadamente zero, os pontos foram selecionados adequadamente. O resultado da aplicação do teste, está apresentado na Tabela 7.17.

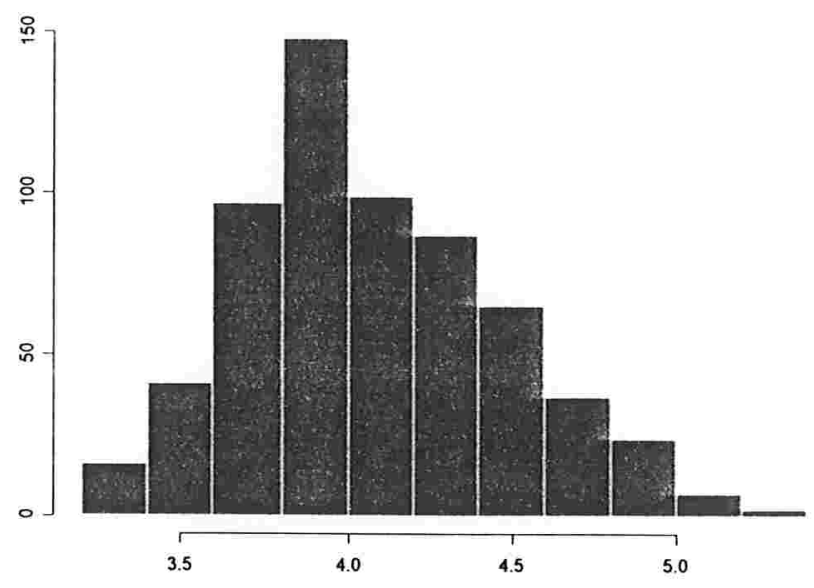

Figura 7.24 - Histograma de LPM10.

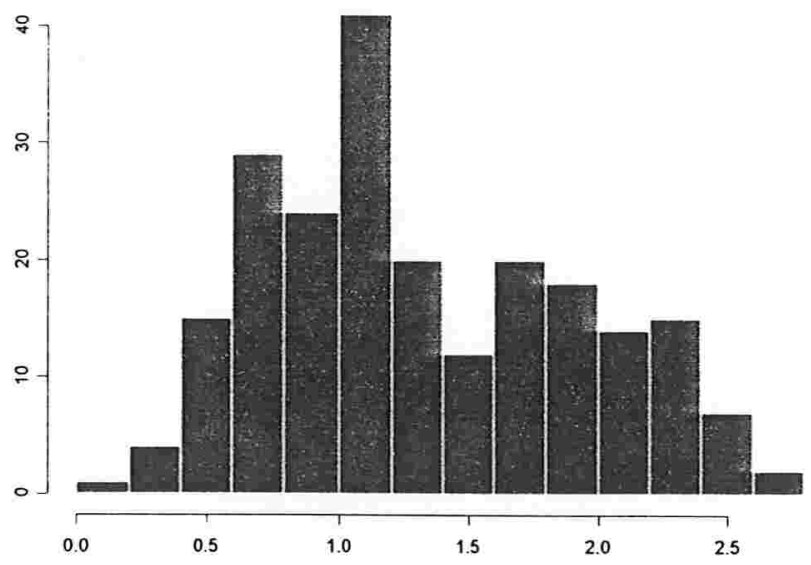

Figura 7.25 - Histograma da função $h$ para LPM10. 
Tabela 7.15 - Pontos selecionados para o teste de Poggi e Portier

\begin{tabular}{cccc}
\hline $\mathrm{T}^{*}$ & $\mathrm{X}_{\mathrm{t}}$ & $\mathrm{X}_{\mathrm{t}-1}$ & $\mathrm{X}_{\mathrm{t}-2}$ \\
\hline 341 & 3,83 & 3,95 & 4,35 \\
359 & 4,18 & 4,43 & 4,40 \\
328 & 4,43 & 4,37 & 4,23 \\
557 & 4,24 & 3,97 & 3,77 \\
\hline
\end{tabular}

Tabela 7.16 - Função h e distância entre os pontos selecionados (D)

\begin{tabular}{cccc}
\hline $\mathrm{T}^{*}$ & $\mathrm{~h}$ & Pontos & $\mathrm{D}$ \\
\hline 341 & 0,65 & $(341,359)$ & 0,0006 \\
359 & 0,85 & $(341,328)$ & 0,0004 \\
328 & 1,11 & $(341,557)$ & 0,0001 \\
557 & 1,20 & $(359,328)$ & 0,0059 \\
& & $(359,557)$ & 0,0003 \\
& & $(328,557)$ & 0,0007 \\
\hline
\end{tabular}

Os testes de linearidade e normalidade de Subba Rao e Gabr foram aplicados nas 7 freqüências principais dadas pelas colunas (3) e (4) da Tabela 5.1, sendo que em torno de cada freqüências principal foi selecionada uma amostra de 21 freqüências satisfazendo (5.8) e (5.9) com $r=5$. Os resultados das aplicações dos testes estão nas Tabelas 7.17 e 7.18 .

Nos testes de normalidade e linearidade de Brillinger e Irizarry em que a série foi particionada em 46 subséries de tamanho 16 obtivemos 29 frequências em que o espectro e o bi-espectro foram calculados. Os níveis descritivos associados aos testes estão nas Tabelas 7.17 e 7.18. Nas Figuras 7.26 e 7.27 estão apresentados os níveis descritivos associados aos testes de normalidade e linearidade. Com relação ao teste de linearidade, $27,58 \%$ dos niveis descritivos associados ao teste são menores do que 0,05 e $86,20 \%$ dos níveis descritivos, associados ao teste de normalidade, são menores do que 0,05 . 


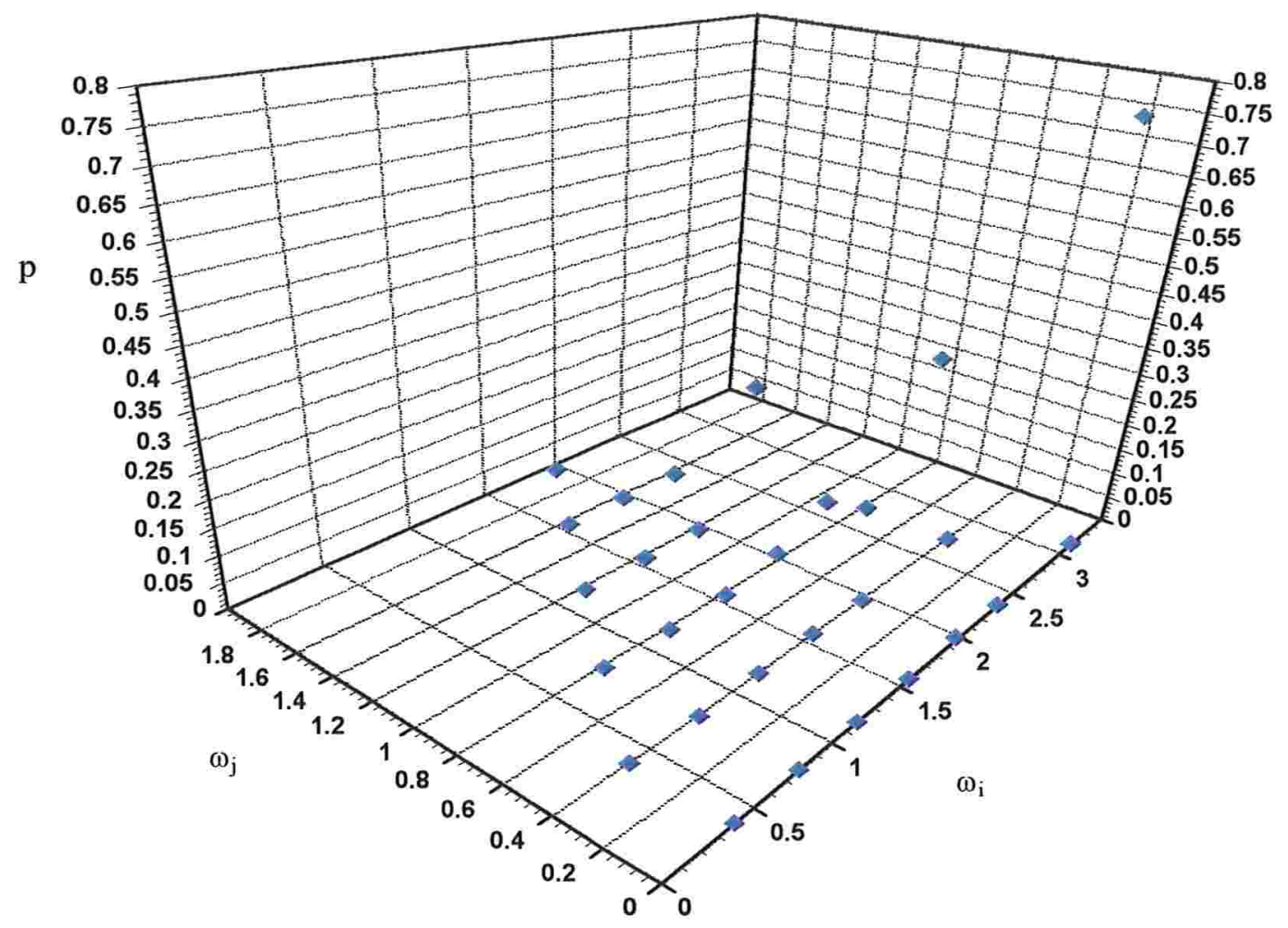

Figura 7.26 - Níveis descritivos associados ao teste de normalidade.

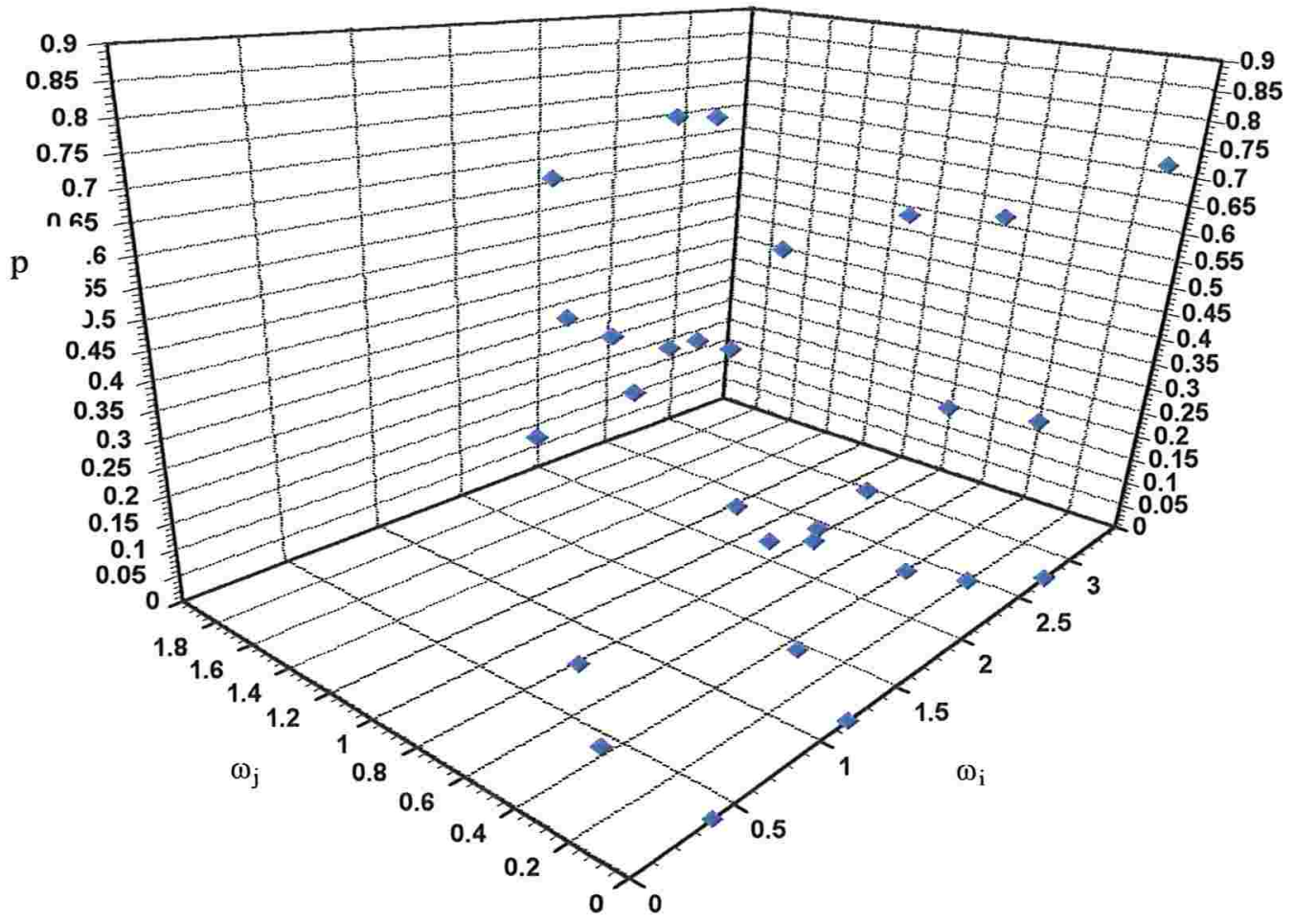

Figura 7.27 - Níveis descritivos associados ao teste de linearidade. 


\section{Conclusão}

Os testes de linearidade e normalidade descritos nesta seção, cujos resultados estão nas Tabela 7.17 e 7.18, indicam que a série LPM10 não possui um comportamento linear nem gaussiano.

Tabela 7.17 - Testes de linearidade para a série LPM10

\begin{tabular}{l|l|c|c|c|c}
\hline & Teste & Estatística & Estimativa & $\mathrm{p}$ & $\begin{array}{c}\text { percentual } \\
\text { de } \mathrm{p}<0,05\end{array}$ \\
\hline & $\begin{array}{l}\text { McLeod e } \\
\mathrm{Li}\end{array}$ & $\mathrm{Q}$ & 171,4 & 0,0000 & - \\
$\begin{array}{l}\text { Domínio do } \\
\text { tempo }\end{array}$ & Tsay & $\mathrm{F}$ & 3,80 & 0,0009 & - \\
\hline $\begin{array}{l}\text { Poggi e } \\
\text { Portier }\end{array}$ & $\mathrm{T}_{\mathrm{q}}(\mathrm{n})$ & $2,18 \times 10^{134}$ & 0,0000 & - \\
$\begin{array}{l}\text { Domínio da } \\
\text { freqüência }\end{array}$ & $\begin{array}{l}\text { Subba Rao e } \\
\text { Gabr } \\
\text { Brillinger e } \\
\text { Irizarry }\end{array}$ & $\mathrm{X}^{*}\left(\omega_{\mathrm{i}}, \omega_{\mathrm{j}}\right)$ & - & - & 27,58 \\
\hline
\end{tabular}

p: nível descritivo associado ao teste

Tabela 7.18 - Testes de normalidade no domínio da freqüência para a série LPM10

\begin{tabular}{l|c|c|c|c}
\hline Teste & Estatística & Estimativa & $\mathrm{p}$ & $\% \mathrm{p}<0,05$ \\
\hline Subba Rao e Gabr & $\Im_{1}$ & 47,97 & 0,0000 & - \\
Brillinger e Irizarry & $\hat{\gamma}_{\mathrm{B}}{ }^{2}\left(\omega_{1}, \omega_{2}\right)$ & - & - & 86,20 \\
\hline
\end{tabular}

p: nível descritivo associado ao teste 


\subsection{Temperatura mínima (TEMP)}

\subsubsection{Análise descritiva e transformação}

A série TEMP é apresentada na Figura 7.28 , podemos notar uma não estacionário homogênea da série, uma vez que as temperaturas mínimas, em geral, decresceram de março a julho. Pela Tabela 7.19, dos dados mensais da série TEMP, notamos que nos meses de janeiro, fevereiro e dezembro ocorreram as maiores médias das temperaturas mínimas para os anos de 1996 e 1997. Para eliminar este comportamento foi aplicada à série uma diferença de ordem 1. A série transformada está apresentada na Figura 7.29. Podemos verificar que a transformação estabilizou a média da série.

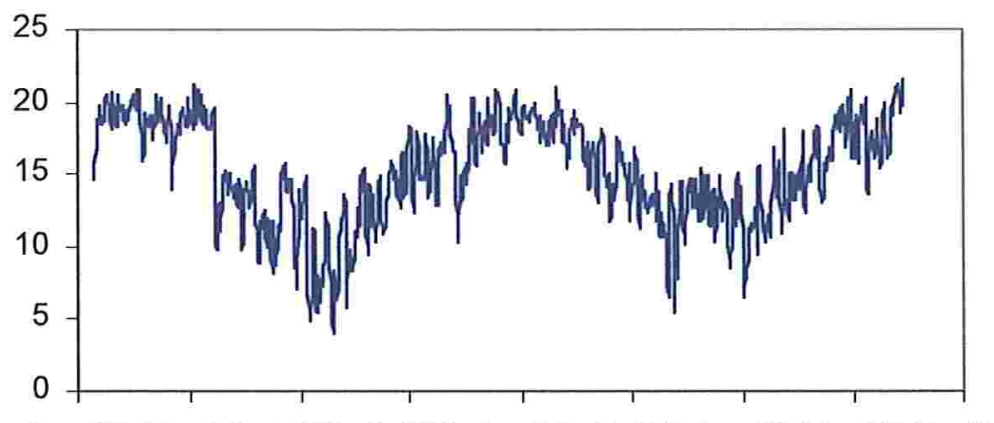

Dez/95 Mar/96 Jul/96 Out/96 Jan/97 Abr/97 Ago/97 Nov/97 Fev/98

Figura 7.28 - Série TEMP. 
Tabela 7.19 - Média e desvio padrão mensais calculados a partir dos dados diários da série TEMP

\begin{tabular}{lcc|lcc}
\hline Mês & Média & $\begin{array}{c}\text { Desvio } \\
\text { Padrão }\end{array}$ & Mês & Média & $\begin{array}{l}\text { Desvio } \\
\text { Padrão }\end{array}$ \\
\hline Jan/96 & 19,2 & 1,1 & Jan/97 & 18,8 & 1,1 \\
Fev/96 & 19,0 & 1,2 & Fev/97 & 18,5 & 1,0 \\
Mar/96 & 18,5 & 1,4 & Mar/97 & 16,8 & 1,7 \\
Abr/96 & 16,3 & 3,3 & Abr/97 & 15,1 & 1,8 \\
Mai/96 & 12,9 & 1,8 & Mai/97 & 13,1 & 1,5 \\
Jun/96 & 12,0 & 2,2 & Jun/97 & 11,8 & 2,7 \\
Jul/96 & 9,7 & 2,9 & Jul/97 & 12,5 & 1,6 \\
Ago/96 & 10,2 & 3,1 & Ago/97 & 11,5 & 2,1 \\
Set/96 & 13,2 & 1,8 & Set/97 & 13,9 & 1,7 \\
Out/96 & 15,0 & 1,7 & Out/97 & 15,9 & 1,9 \\
Nov/96 & 15,8 & 2,4 & Nov/97 & 18,0 & 1,7 \\
Dez/96 & 18,2 & 1,6 & Dez/97 & 18,6 & 1,9 \\
\hline
\end{tabular}

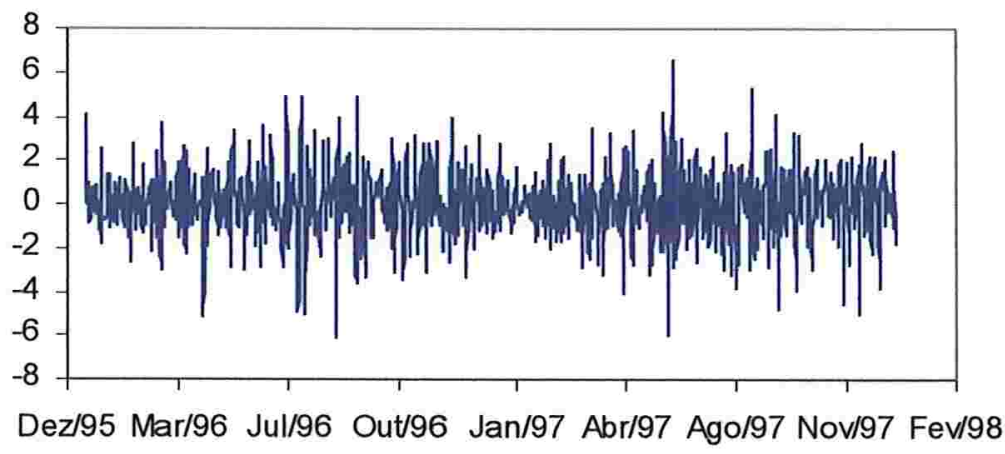

Figura 7.29 - Gráfico da série (1-B)TEMP.

Podemos notar pela Figura 7.30, em que as 730 observações da série transformada foram divididas em 52 subséries de 14 elementos cada e foram calculadas as médias e os desvios padrões de cada segmento, que a diferença aplicada à série TEMP parece ser suficiente. 


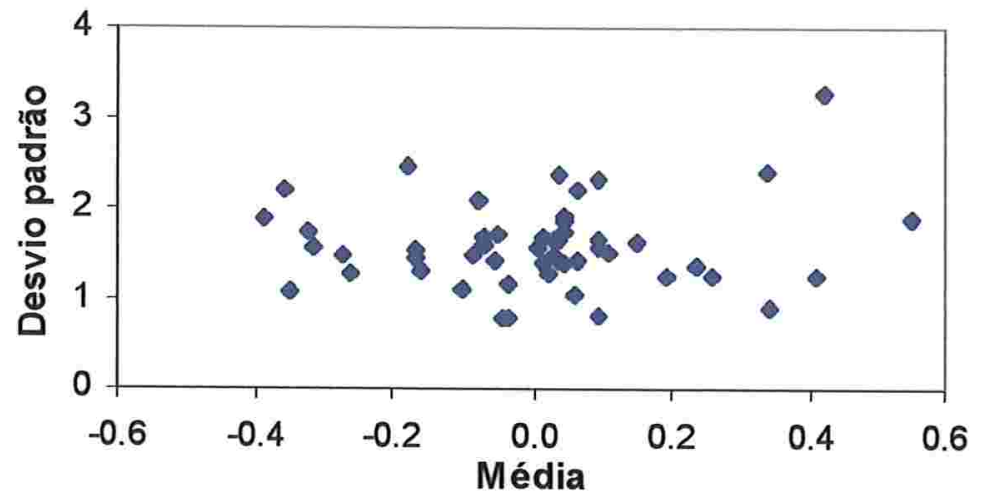

Figura 7.30 - Gráfico do desvio padrão x média de cada uma das 52 subséries para a série (1-B)TEMP.

Analisando a Figura 7.31, em que estão representadas as funções de autocorrelação amostral das séries TEMP e (1-B)TEMP, notamos que a transformação foi adequada e parece ter tornado a série estacionária.
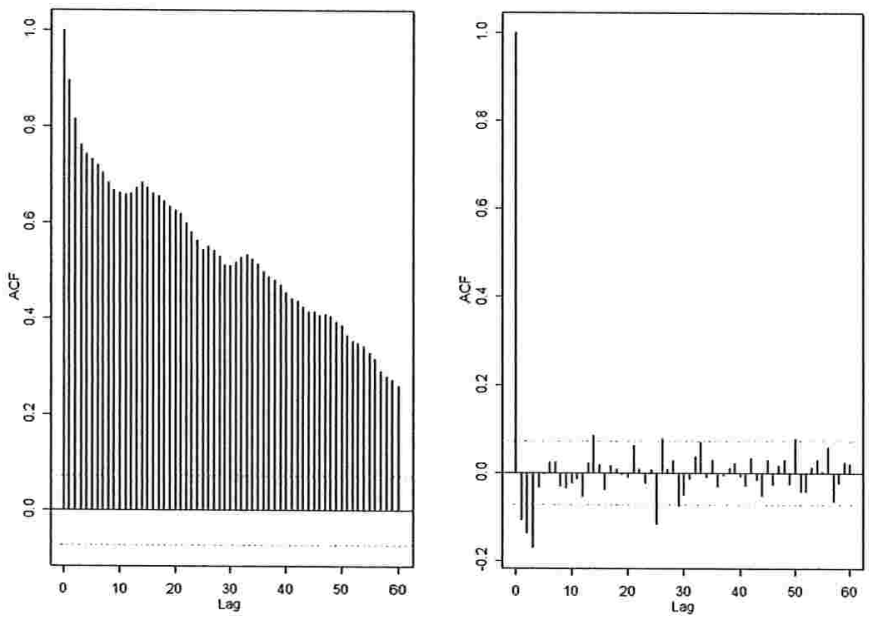

Figura 7.31 - Funções de autocorrelação amostral para as séries TEMP e (1-B)TEMP. 


\subsubsection{Aplicação dos testes de linearidade e normalidade}

Os testes de linearidade e normalidade descritos nesta seção foram aplicados à diferença de ordem 1 da série de temperatura mínima, que passaremos a denotar por (1-B)TEMP.

Para a realização do teste de linearidade de McLeod e Li, foi ajustado um modelo ARMA à série com uma diferença, isto é,

$$
(1-0,63 \mathrm{~B})(1-\mathrm{B}) \mathrm{TEMP}_{\mathrm{t}}=(1-0,89 \mathrm{~B})\left(1-0,11 \mathrm{~B}^{3}\right)\left(1+0,10 \mathrm{~B}^{14}\right)\left(1-0,10 \mathrm{~B}^{25}\right) \mathrm{e}_{\mathrm{t}}
$$

As funções de autocorrelação parcial e autocorrelação amostral do resíduo do modelo dado pela expressão (7.4) estão apresentadas na Figura 7.32. Na Tabela 7.23 está apresentado o resultado da aplicação do teste.
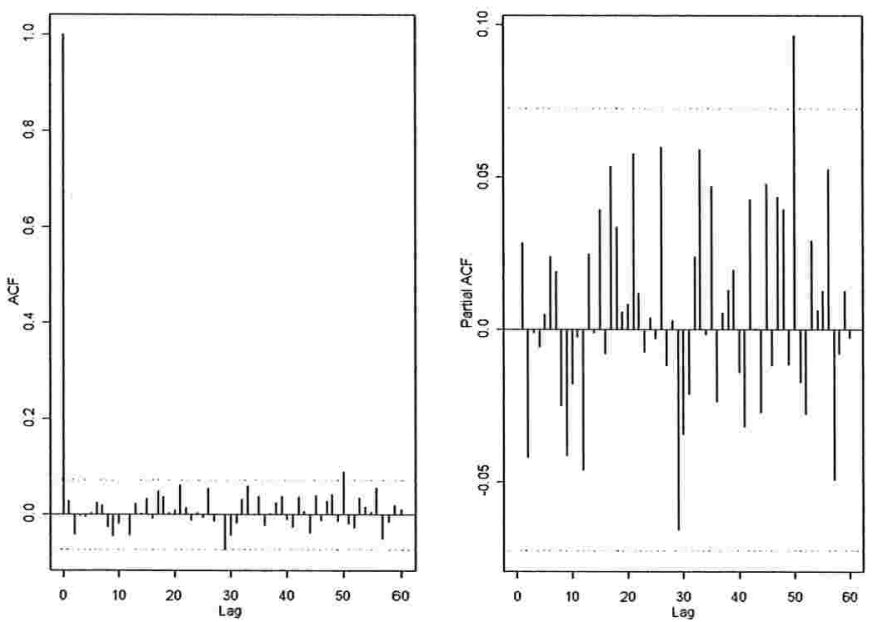

Figura 7.32 - Funções de autocorrelação e autocorrelação parcial amostral do resíduo do modelo (7.4).

Com relação ao teste de linearidade de Tsay os valores de AIC e SBC obtidos a partir dos modelos auto-regressivos ajustados estão na Tabela 7.20, 
notamos que o modelo que se ajustou bem à série (1-B)TEMP foi um AR(4), entretanto, esta escolha acarretaria no ajuste de uma regressão multivariada de ordem 10 no terceiro passo do teste, desta forma foi escolhido como um bom modelo o $\mathrm{AR}(3)$. O nível descritivo associado ao teste está na Tabela 7.23.

Tabela 7.20 - Valores de AIC e SBC para a série (1-B)TEMP

\begin{tabular}{ccc}
\hline AR & AIC & SBC \\
\hline 1 & 2757 & 2762 \\
2 & 2742 & 2751 \\
$\mathbf{3}$ & $\mathbf{2 7 1 1}$ & $\mathbf{2 7 2 5}$ \\
4 & 2703 & 2721 \\
5 & 2698 & 2721 \\
6 & 2697 & 2724 \\
7 & 2698 & 2730 \\
8 & 2697 & 2734 \\
9 & 2695 & 2737 \\
10 & 2694 & 2740 \\
11 & 2691 & 2742 \\
12 & 2681 & 2736 \\
13 & 2679 & 2738 \\
14 & 2681 & 2745 \\
15 & 2682 & 2751 \\
\hline
\end{tabular}

Para a realização do teste de linearidade de Poggi e Portier devemos escolher a ordem do modelo auto-regressivo que melhor se ajustou à série e para isto foi utilizado o mesmo critério do teste de Tsay, ou seja, um AR(3). Para a realização do teste é necessário fazer a escolha de $\mathrm{q}$ pontos do suporte da distribuição h. O histograma de (1-B)TEMP está representado na Figura 7.33, como esta distribuição é aproximadamente gaussiana, a função $\mathrm{h}$ foi calculada nos pontos $\left(X_{t}, X_{t-1}, X_{t-2}\right)$ tal que $]-23,0<X_{t-i}<23,0[$, para $i=0,1,2$. A Tabela 7.21 apresenta os pontos selecionados para o teste e o tempo em que a observação ocorreu $\left(\mathrm{T}^{*}\right)$. Estes pontos, que possuem alta densidade, foram selecionados pelo 
histograma da função h que está apresentado na Figura 7.34. A Tabela 7.22 apresenta o valor da função h para cada ponto selecionado e a distância entre estes pontos; como esta distância é aproximadamente zero os pontos foram selecionados adequadamente. O resultado da aplicação do teste, está apresentado na Tabela 7.23 .

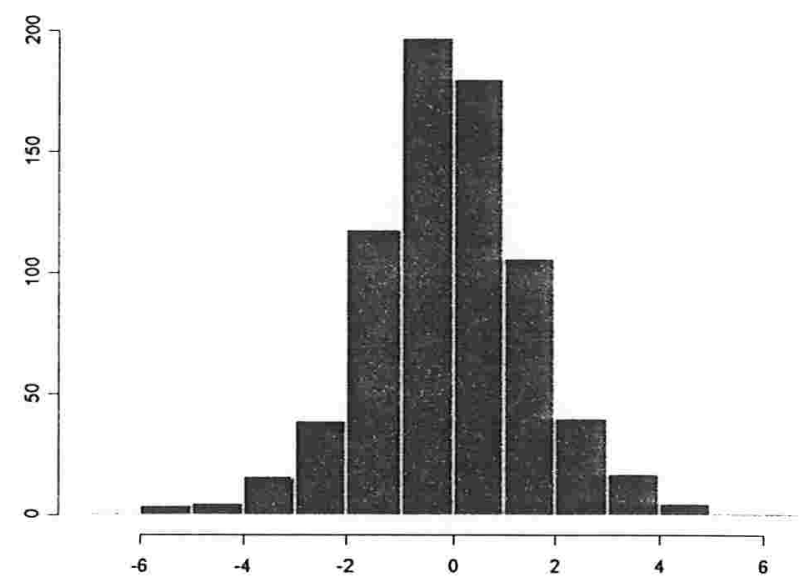

Figura 7.33 - Histograma de (1-B)TEMP.

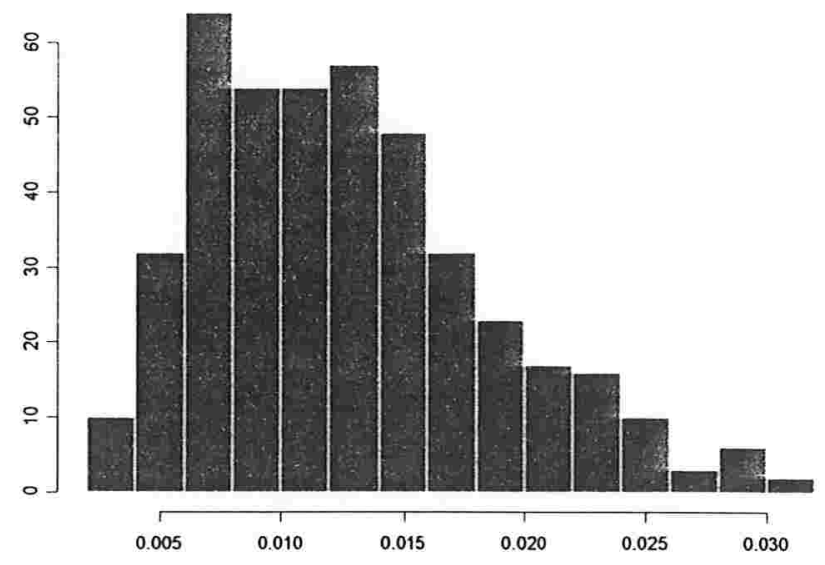

Figura 7.34 - Histograma da função h para (1B)TEMP. 
Tabela 7.21 - Pontos selecionados para o teste de Poggi e Portier

\begin{tabular}{cccc}
\hline $\mathrm{T}^{*}$ & $\mathrm{X}_{\mathrm{t}}$ & $\mathrm{X}_{\mathrm{t}-1}$ & $\mathrm{X}_{\mathrm{t}-2}$ \\
\hline 650 & 1,2 & 1,0 & $-1,9$ \\
343 & 1,8 & $-0,8$ & $-0,5$ \\
666 & 0,1 & 2,0 & 1,0 \\
262 & 0,3 & 1,0 & 0,6 \\
\hline
\end{tabular}

Tabela 7.22 - Função $h$ e distância entre os pontos selecionados (D)

\begin{tabular}{cccc}
\hline $\mathrm{T}^{*}$ & $\mathrm{~h}$ & Pares & $\mathrm{D}$ \\
\hline 650 & 0,00605 & $(650,343)$ & $2,13 \times 10^{-11}$ \\
343 & 0,00787 & $(650,666)$ & $9,62 \times 10^{-11}$ \\
666 & 0,00906 & $(650,262)$ & $2,92 \times 10^{-11}$ \\
262 & 0,01109 & $(343,666)$ & $1,33 \times 10^{-11}$ \\
& & $(343,262)$ & $4,50 \times 10^{-11}$ \\
& & $(666,262)$ & $9,30 \times 10^{-11}$ \\
\hline
\end{tabular}

Os testes de linearidade e normalidade de Subba Rao e Gabr foram aplicados nas 7 frequeências principais dadas pelas colunas (3) e (4) da Tabela 5.1, sendo que em torno de cada freqüências principal foi selecionada uma amostra de 21 frequêencias satisfazendo (5.8) e (5.9) com r=5. Os resultados das aplicações dos testes estão nas Tabelas 7.23 e 7.24 .

Nos testes de normalidade e linearidade de Brillinger e Irizarry a série foi particionada em 46 subséries de tamanho 16, desta forma, obtivemos 29 freqüências em que o espectro e o bi-espectro foram calculados. Os níveis descritivos associados aos testes estão nas Tabelas 7.23 e 7.24. Os níveis descritivos associados aos testes de normalidade e linearidade estão apresentados nas Figuras 7.35 e 7.36. Com relação ao teste de linearidade $24,13 \%$ dos níveis descritivos são menores do que 0,05 e $41,37 \%$ dos níveis descritivos associados ao teste de normalidade são menores do que 0,05 . 


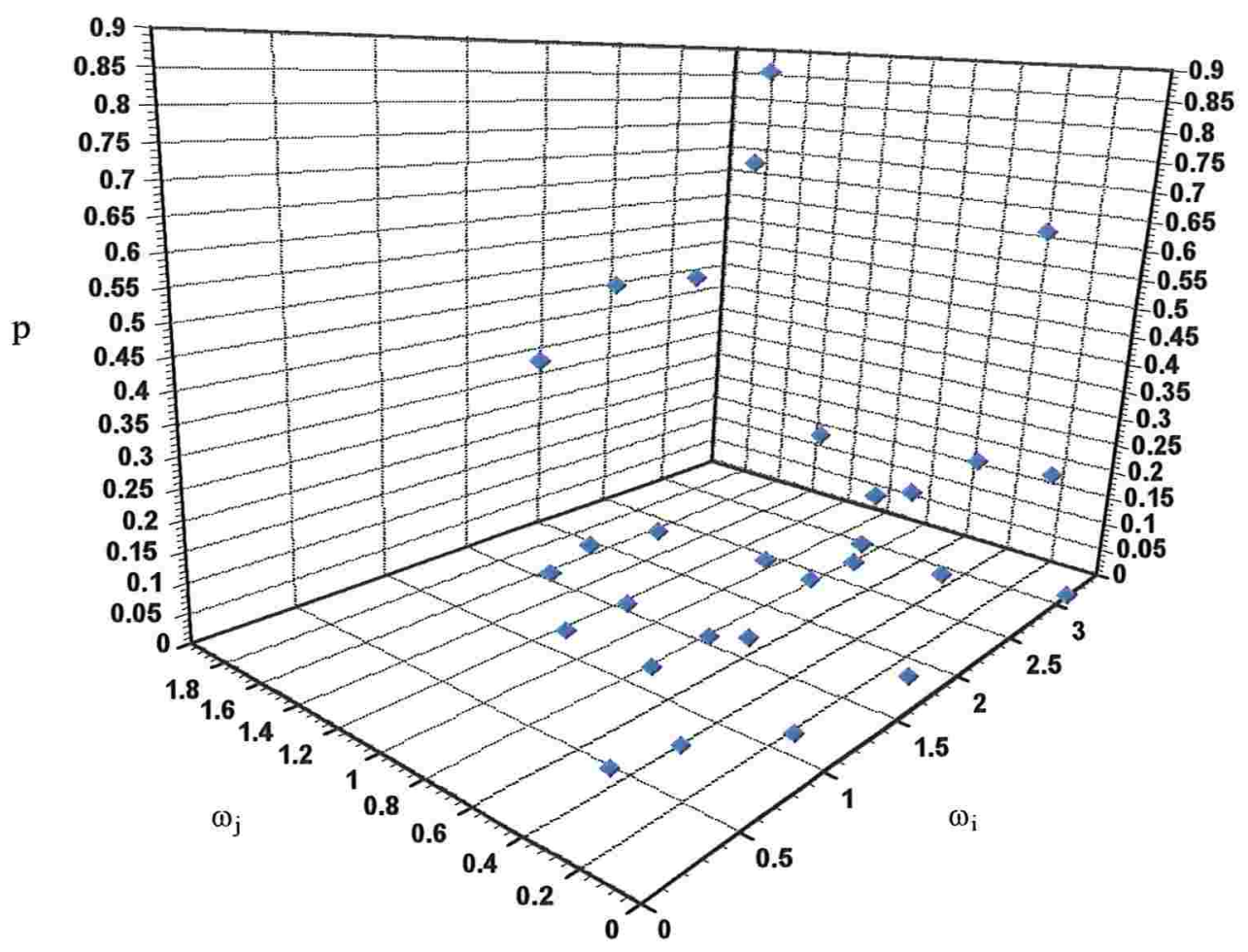

Figura 7.35 - Niveis descritivos associados ao teste de normalidade.

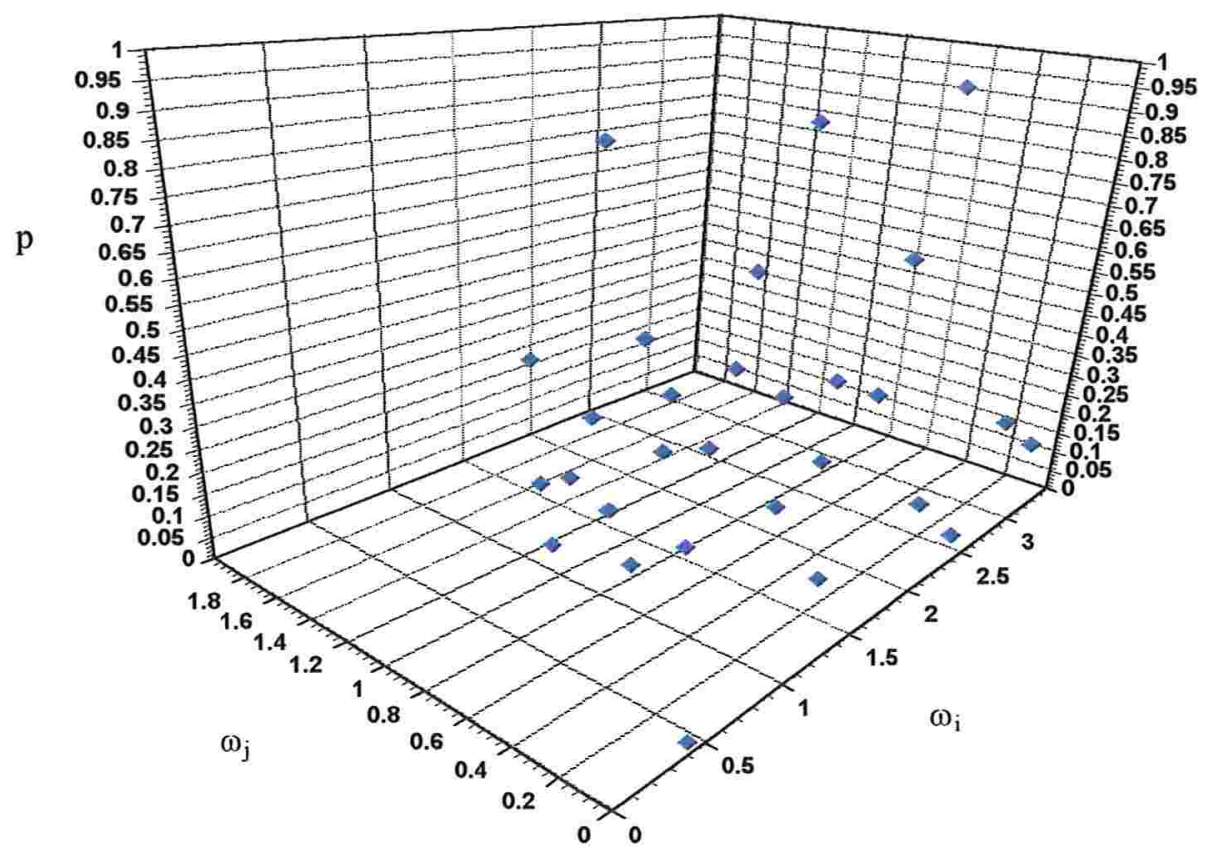

Figura 7.36 - Níveis descritivos associados ao teste de linearidade. 


\section{Conclusão}

Os testes de linearidade e normalidade descritos nesta seção, cujos resultados estão nas Tabelas 7.23 e 7.24 , indicam que a série não possui um comportamento linear nem gaussiano.

Tabela 7.23 - Testes de linearidade para a série (1-B)TEMP

\begin{tabular}{l|l|c|c|c|c}
\hline & Teste & Estatística & Estimativa & $\mathrm{p}$ & $\begin{array}{c}\text { percentual } \\
\mathrm{de} \mathrm{p}<0,05\end{array}$ \\
\hline & $\begin{array}{l}\text { McLeod e } \\
\mathrm{Li} \\
\mathrm{T} \text { say }\end{array}$ & $\mathrm{Q}$ & 81,73 & 0,032 & - \\
$\begin{array}{l}\text { Domínio do } \\
\text { tempo }\end{array}$ & $\begin{array}{l}\text { Poggi e } \\
\text { Portier }\end{array}$ & $\mathrm{T}_{\mathrm{q}}(\mathrm{n})$ & 12,63 & 0,0132 & - \\
\hline $\begin{array}{l}\text { Domínio da } \\
\text { freqüência }\end{array}$ & $\begin{array}{l}\text { Subba Rao e } \\
\text { Gabr } \\
\text { Brillinger e } \\
\text { Irizarry }\end{array}$ & $\mathrm{X}^{*}\left(\omega_{\mathrm{i}}, \omega_{\mathrm{j}}\right)$ & - & - & 24,13 \\
\hline
\end{tabular}

p: nível descritivo associado ao teste

Tabela 7.24 - Testes de normalidade no domínio da freqüência para a série (1B) TEMP

\begin{tabular}{l|c|c|c|c}
\hline Teste & Estatística & Estimativa & $\mathrm{p}$ & $\% \mathrm{p}<0,05$ \\
\hline Subba Rao e Gabr & $\mathfrak{I}_{1}$ & 26,39 & 0,0000 & - \\
Brillinger e Irizarry & $\hat{\gamma}_{\mathrm{B}}{ }^{2}\left(\omega_{1}, \omega_{2}\right)$ & - & - & 41,37 \\
\hline
\end{tabular}

p: nível descritivo associado ao teste 


\subsection{Considerações finais}

Este trabalho teve como objetivo descrever e aplicar a algumas séries reais alguns testes de linearidade e normalidade. Descreveremos a seguir algumas das vantagens e desvantagens de cada um dos testes descritos nos Capítulos 5 e 6 e aplicados nas série reais de poluição, temperatura e óbitos da cidade de São Paulo:

i) Os testes de linearidade no domínio do tempo de McLeod e Li e de Tsay têm a vantagem de possuírem uma aplicação fácil mas a desvantagem de serem paramétricos, ou seja, requer o ajustamento de um modelo a série analisada;

ii) O teste de linearidade no domínio do tempo de Poggi e Portier possui a vantagem de ser não paramétrico, entretanto, possui a desvantagem de ter uma aplicação trabalhosa, pois necessita da escolha de pontos do suporte da distribuição do processo e esta escolha não é trivial;

iii) Os testes de linearidade e normalidade no domínio da freqüência de Subba Rao e Gabr possuem as vantagens de serem não paramétricos e de fácil aplicação; entretanto, possuem a desvantagem de levar um tempo computacional muito grande no cálculo do bi-espectro para uma grande quantidade de freqüências, por este motivo o programa foi desenvolvido em Turbo Pascal;

iv) Os testes de linearidade e normalidade no domínio da freqüência de Brillinger e Irizarry possuem as vantagens de ter uma aplicação fácil e são executados rapidamente pelo S-plus. 
Bibliografia

\section{Bibliografia}

Anderson, T. W. (1958). An Introduction to Multivariate Analysis. Wiley, New York.

Andel, J. (1976). Autoregressive series with random parameters. Math. Operationsforsch. u. Statist., 7, 735-741.

Ashley, R. A., Patterson, D. M. e Hinich, M. J. (1986). A diagnostic test for nonlinearity serial dependence in time series fitting errors. Journal of Time Series Analysis. 7(3), 165-178.

Bollerslev, T. (1986). Generalised autoregressive conditional heteroscedasticity. Journal of Econometrics, 51, 307-327.

Bollerslev, T, Chou, R.Y. e Kroner, K.F. (1992). Arch modeling in finance: a review of the theory and empirical evidence. Journal of Econometrics, 52, 5-59.

Box, G. E. P., Jenkins, G. M. e Reinsel, G. C. (1994). Time Series Analysis, Forecasting and Control, San Francisco, Holden-Day.

Brillinger, D.R. (1965). An introduction to polyspectra. Ann. Math. Statist., 36, 1351-1374.

Brillinger, D.R. e Irizarry, R.A. (1998). An investigation of the second and higherorder spectra of music. Signal Processing 65, 161-179.

Brillinger, D.R. e Rosenblatt, M. (1967). Computation and interpretation of k-th order spectra. Spectral Analysis of Time Series. John Wiley, 189-232.

Brockett, P. L.,Hinich, M. J. e Patterson, D.M. (1988). Bispectral based tests for the detection of gaussianity and linearity in time series. Journal of the American Statistical Association. 83, 657-664.

Chan, W.S. e Tong, H. (1986). On tests for nonlinearity in time series analysis Journal of Forecasting, 5, 217-228.

Davies, N. e Petruccelli, J. D. (1985). Experience with Detecting Non-linearity in Time Series: Identification and Diagnostic Checking. Res. Rep., MSOR/8/85, Trent Polytechnic, Nottingham.

Duflo, M. (1990). Méthodes récursives aléatoires. Paris: Masson. 
Dunis, C. e Zhou, B. (1998). Nonlinear Modelling of High Frequency Financial Time Series. John Wiley \& Sons.

Engle, R.F. (1982). Autoregressive conditional heteroscedasticity with estimates of the variance of the United Kindom inflation. Econometrica, 50, 987-1007.

Giri, N.(1965). On the complex analogues of $\mathrm{T}^{2}$ and $\mathrm{R}^{2}$ tests. Ann. Math. Statist., $36,664-670$.

Granger, C. W. J. e Andersen, A. P. (1978). An Introduction to Bilinear Time Series Models. Vandenhock and Ruprecht, Göttingen.

Haggan, V. e Ozaki, T. (1981). Modelling non-linear randon vibrations using an amplitude-dependent autoregressive time series model. Biometrika, 68, 189-196.

Hinich, M. J. (1982). Testing for gaussianity and linearity of stationary time series. Journal of Time Series Analysis. 3, 169-76.

Keenan, D.M. (1985). A Tukey non-additivity-type test for time series nonlinearity. Biometrika, 72, 39-44.

Khatri, C.G.(1965). Classical statistical analysis based on a certain multivariate complex Wishart distribution. Ann. Math. Statist., 36, 98-107.

Lawrance, A. J. and Lewis, P. A. W. (1980). The exponencial autoregressive moving average EARMA(p, q) process, Journal of the Royal Statistical Society, B, $42,150-161$.

Luukkonen, R., Saikkonen,P., e Teräsvirta, T. (1988). Testing linearity in univariate time series models. Scand. J. Stat., 15,161-175.

Maravall, A. (1983). An application of nonlinear time series forecasting. J. Bus. Econ. Stat., 1,66-74.

McLeod, A. I. and Li,W.K. (1983). Diagnostic checking ARMA time series models using squared-residual autocorrelations. Journal of Time Series Analysis, 4, 269273.

Mohler, R.R. (1973). Bilinear Control Processes, Academic Press, New York and London.

Nicholls, D.F. e Quinn, B.G. (1982). Random Coefficient Autoregressive Models: An Introduction. Springer-Verlag, Berlin. 
Ozaki, T. (1978). Non-linear models for non-linear random vibrations . Technical Report no. 92, Department of Mathematics, University of Manchester Institute of Science and Technology, UK.

Poggi, J. e Portier, B. (1997). A test of linearity for functional autoregressive models. Journal of Time Series Analysis, 18, 616-639.

Priestley, M. B. (1988). Non-linear and Non-stationary Time Series Analysis. Academic Press.

Rosenblatt, M. (1956). Remarks on some nonparametrics estimates of a density function. Ann. Math. Stat. 1, 4-27.

Ruberti, A., Isidori, A. e d'Allessandro, P.(1972). Theory of Bilinear Dynamical Systems, Sringer Verlag, Berlim.

Subba Rao, T. e Gabr,M.M. (1980). A test for linearity of stationary time series. Journal of Time Series Analysis, 1, 145-158.

Subba Rao, T. (1981). On the theory of bilinear models. Jounal of the Royal Statistical Society. Ser. B,43,244-255.

Subba Rao, T. e Gabr, M. M. (1984). An Introduction to Bispectral Analysis and Bilinear Time Series Models. Springer-Verlag, Berlin.

Tong, H. (1977). Discussion of a paper by A. J. Lawrance e N. T. Kottegoda, Jounal of the Royal Statistical Society, A, 140, 34-35.

Tong, H (1990). Non-linear Time Series. A Dynamical System Approach. Oxford Science Publications.

Tukey, J.W. (1949). One degree of freedom for non additivity. Biometrics 5, 23242.

Tsay, R. S. (1986). Nonlinearity tests for time series. Biometrika,73,461-466. 
APÊNDICE A

DADOS 
Tabela A.1 - Dados de temperatura da cidade de São Paulo $(*)$

\begin{tabular}{|c|c|c|c|c|c|c|c|c|c|}
\hline 14,60 & 19,30 & 18,20 & 13,00 & 11,70 & 12,00 & 12,00 & 12,60 & 14,90 & 13,90 \\
\hline 18,70 & 20,40 & 18,00 & 11,10 & 11,20 & 12,00 & 11,40 & 11,00 & 14,60 & 15,80 \\
\hline 18,90 & 20,90 & 17,80 & 11,70 & 11,20 & 13,00 & 12,40 & 10,80 & 14.70 & 14,40 \\
\hline 18,90 & 19,30 & 18,70 & 12,60 & 11,60 & 13,00 & 13,60 & 11,40 & 17,80 & 16,00 \\
\hline 19,80 & 19,80 & 19,60 & 14,20 & 12,50 & 13,50 & 13,10 & 12,40 & 16,20 & 16,60 \\
\hline 19,00 & 17,20 & 19,00 & 15,30 & 11,80 & 14,80 & 7,00 & 12,70 & 13,90 & 18,20 \\
\hline 18,40 & 16,00 & 19,10 & 14,90 & 10,00 & 13,90 & 5,80 & 13,80 & 13,40 & 17,30 \\
\hline 19,10 & 16,40 & 19,00 & 14,50 & 11,90 & 9,00 & 9,80 & 14,20 & 14,20 & 19,90 \\
\hline 18,70 & 19,20 & 18,30 & 15,00 & 10,60 & 4,90 & 8,30 & 15,70 & 13,80 & 20,20 \\
\hline 19,20 & 18,00 & 18,30 & 13,60 & 11,70 & 5,40 & 9,20 & 16,00 & 16.60 & 16,90 \\
\hline 20,00 & 18,60 & 20,20 & 15,00 & 10,90 & 6,30 & 8,40 & 15,40 & 15,70 & 16,40 \\
\hline 20,50 & 18,80 & 18,60 & 14,60 & 8.20 & 11,20 & 9,20 & 15,40 & 17,60 & 15,60 \\
\hline 20,00 & 18,30 & 19,30 & 13,50 & 11,80 & 11,10 & 11,00 & 14,60 & 14,60 & 17,40 \\
\hline 19,90 & 19,00 & 18,20 & 13,40 & 11,00 & 11,00 & 11,00 & 15,30 & 12,80 & 17,90 \\
\hline 20,00 & 19,00 & 18,60 & 13,80 & 10,40 & 6,00 & 10,60 & 14,10 & 12,80 & 19,20 \\
\hline 18,20 & 18,60 & 21,20 & 14,40 & 8,70 & 5,40 & 12,70 & 13,20 & 15,60 & 17,30 \\
\hline 20,70 & 17,40 & 20,70 & 13,80 & 10,30 & 8,00 & 11,40 & 13,60 & 17,20 & 16,50 \\
\hline 20,30 & 19,20 & 18,50 & 12,70 & 10,80 & 7,20 & 13,60 & 14,60 & 16,30 & 17,40 \\
\hline 19,60 & 20,44 & 20,90 & 14,60 & 11,70 & 6,20 & 14,80 & 12,60 & 17.00 & 18,60 \\
\hline 18,90 & 19,50 & 19,80 & 14,00 & 11,10 & 7,80 & 13,80 & 15,50 & 16,50 & 18,20 \\
\hline 18,30 & 18,60 & 18,80 & 12,50 & 14,20 & 7,20 & 14,60 & 16,50 & 16,40 & 17,10 \\
\hline 19,20 & 19,30 & 20,40 & 9,80 & 15,60 & 8,40 & 15,40 & 13,40 & 19,30 & 20,20 \\
\hline 20,50 & 19,30 & 19,40 & 10,40 & 14,70 & 9,00 & 13,00 & 13,90 & 20,40 & 19,30 \\
\hline 19,40 & 20,30 & 18,40 & 13,70 & 15,80 & 12,40 & 9,50 & 14,30 & 19.40 & 19,00 \\
\hline 19,30 & 18,20 & 19,70 & 14,00 & 14,60 & 11,00 & 14,40 & 16,20 & 19.80 & 18,30 \\
\hline 19,70 & 18,40 & 19,20 & 14,50 & 14,40 & 10,00 & 14,40 & 17,50 & 18.90 & 19,00 \\
\hline 19,30 & 17,60 & 19,20 & 14,10 & 13,80 & 9,60 & 14,20 & 18,30 & 16.80 & 17,70 \\
\hline 18,50 & 18,70 & 18,50 & 13,70 & 14,70 & 7,20 & 11,70 & 18,10 & 16,80 & 18,20 \\
\hline 19,40 & 17,20 & 18,10 & 12,60 & 14,70 & 5,90 & 11,50 & 14,60 & 16,50 & 19,80 \\
\hline 18,50 & 19,60 & 18,20 & 13,40 & 13,60 & 4,00 & 13,60 & 12,70 & 15,30 & 20,80 \\
\hline 18,90 & 19,70 & 18,30 & 14,60 & 12,60 & 5,60 & 13,60 & 12,40 & 14,40 & 20,10 \\
\hline 18,80 & 17,70 & 18,20 & 15,60 & 9,70 & 8,30 & 10,30 & 13,50 & 11.80 & 20,70 \\
\hline 20,00 & 16,80 & 19,30 & 15,10 & 8,60 & 7,10 & 10,40 & 16,20 & 10,40 & 20,00 \\
\hline 20,10 & 13,90 & 14,30 & 12,10 & 8.80 & 6,40 & 12,30 & 18,00 & 14.30 & 18,40 \\
\hline 19,90 & 17,60 & 10,90 & 11,20 & 7.10 & 7,00 & 13,70 & 17,10 & 14.80 & 17,40 \\
\hline 20,20 & 16,50 & 9,80 & 9,80 & 11,90 & 10,00 & 14,80 & 14,70 & 13.00 & 17,10 \\
\hline 20,40 & 18,40 & 12,30 & 8,80 & 14.00 & 11,30 & 13,90 & 14,80 & 13.60 & 16,60 \\
\hline
\end{tabular}

$\left(^{*}\right)$ ler por colunas 
Tabela A.2 - Dados de temperatura da cidade de São Paulo (continuação)

\begin{tabular}{|c|c|c|c|c|c|c|c|c|c|}
\hline 15,80 & 17,40 & 17,30 & 14,60 & 6,50 & 13,20 & 10,60 & 15,00 & 16,90 & 17,40 \\
\hline 18,50 & 18,00 & 16,90 & 13,30 & 8,20 & 12,20 & 11,60 & 13,60 & 18,90 & 16,20 \\
\hline 17,30 & 17,00 & 14,00 & 15,80 & 12,40 & 11,50 & 11,00 & 13,20 & 19,00 & 16,60 \\
\hline 18,70 & 18,10 & 14,40 & 11,80 & 14,40 & 12,80 & 11,40 & 14,80 & 18,80 & 18,80 \\
\hline 19,60 & 19,00 & 15,60 & 14,30 & 14,10 & 13,90 & 12,60 & 14,50 & 18,20 & 18,50 \\
\hline 19,10 & 18,60 & 16,90 & 14,40 & 11,50 & 12,00 & 12,40 & 14,30 & 18,20 & 16,20 \\
\hline 18,90 & 17,20 & 17,20 & 14,40 & 5,60 & 10,40 & 9,50 & 14,00 & 19,60 & 15,40 \\
\hline 19,60 & 19,20 & 16,10 & 14,40 & 7,20 & 11,80 & 14,80 & 15,50 & 18,00 & 16,00 \\
\hline 19,50 & 18,30 & 13,60 & 16,80 & 9,50 & 13,40 & 15,50 & 13,90 & 18,90 & 18,20 \\
\hline 19,70 & 21,10 & 14,60 & 15,40 & 7,90 & 12,60 & 14,30 & 17,00 & 19,00 & 19,70 \\
\hline 20,80 & 19,10 & 13,00 & 13,80 & 14,50 & 14,80 & 11,80 & 17,90 & 19,70 & 19,40 \\
\hline 19,50 & 20,10 & 16,30 & 11,20 & 11,60 & 12,60 & 11,20 & 14,00 & 18,70 & 20,00 \\
\hline 18,50 & 19,00 & 17,60 & 14,60 & 14,40 & 12,80 & 12,40 & 12,40 & 16,90 & 16,20 \\
\hline 18,60 & 19,30 & 18,00 & 13,30 & 14,50 & 11,80 & 10,40 & 12,50 & 18,10 & 16,20 \\
\hline 17,80 & 19,00 & 18,10 & 14,80 & 12,00 & 12,80 & 11,00 & 15,60 & 20,10 & 16,50 \\
\hline 19,50 & 17,30 & 17,60 & 14,00 & 10,90 & 13,00 & 11,60 & 14.80 & 20,20 & 17,90 \\
\hline 19,40 & 18,40 & 14,80 & 13,70 & 10,20 & 12,50 & 12,20 & 14,50 & 20,80 & 18,60 \\
\hline 19,70 & 17,30 & 14,90 & 12,60 & 13,20 & 10,20 & 10,60 & 15,10 & 16,30 & 20,10 \\
\hline 19,40 & 17,10 & 14,40 & 13,00 & 12,40 & 11,60 & 11,30 & 15,60 & 16,10 & 19,10 \\
\hline 18,90 & 15,40 & 14,20 & 12,60 & 13,10 & 8,60 & 12.20 & 16.40 & 17,20 & 21,10 \\
\hline 18,90 & 17,40 & 15,00 & 12,30 & 14,60 & 9,30 & 14,40 & 18,00 & 19,00 & 21,10 \\
\hline 19,00 & 16,10 & 11,80 & 13,10 & 13,80 & 10,00 & 16.80 & 16.10 & 16,20 & 20,80 \\
\hline 18,60 & 18,20 & 12,20 & 13,00 & 14,70 & 13,20 & 13,90 & 17.10 & 16,10 & 20,70 \\
\hline 18,80 & 18,20 & 14,30 & 13,50 & 12,70 & 11,50 & 14,50 & 18.30 & 15,80 & 21,20 \\
\hline 19,60 & 18,90 & 13,20 & 12,80 & 14,70 & 11,60 & 13,40 & 18.10 & 18,00 & 20,30 \\
\hline 19,50 & 18,00 & 14,40 & 13,30 & 13,30 & 13,40 & 15.90 & 15.10 & 19,00 & 19,20 \\
\hline 19,50 & 19,30 & 13,30 & 15,00 & 12,60 & 15,10 & 14,00 & 13.40 & 19,80 & 21,60 \\
\hline 20,00 & 17,80 & 14,40 & 11,80 & 13,50 & 11,90 & 12,30 & 13.00 & 18,70 & 19,80 \\
\hline 19,40 & 18,80 & 17,60 & 13,80 & 12,10 & 12,80 & 10,80 & 13.40 & 18,60 & \\
\hline 18,90 & 18,30 & 17,00 & 11,20 & 11,80 & 14,20 & 14,20 & 15.40 & 18,40 & \\
\hline 18,20 & 18,40 & 16,00 & 10,60 & 13,00 & 10,40 & 18,20 & 15,80 & 20,10 & \\
\hline 19,00 & 18,40 & 16,20 & 11,40 & 15,40 & 9,40 & 13,60 & 15.20 & 15,10 & \\
\hline 17,30 & 18,30 & 16,30 & 12,30 & 14,60 & 6,60 & 12,80 & 15.70 & 13,50 & \\
\hline 18,70 & 17,50 & 16,40 & 11,00 & 12,00 & 8,30 & 13.20 & 16.30 & 16,10 & \\
\hline 18,10 & 16,80 & 15,20 & 10,60 & 13,20 & 7,80 & 11,80 & 16.90 & 17,70 & \\
\hline 18,50 & 15,60 & 15,70 & 10,80 & 14,90 & 9,60 & 13.50 & 15.90 & 18,00 & \\
\hline 17,10 & 16,00 & 14,30 & 8,60 & 14,80 & 11,00 & 15.00 & 15.90 & 16,60 & \\
\hline
\end{tabular}

$(*)$ ler por colunas 


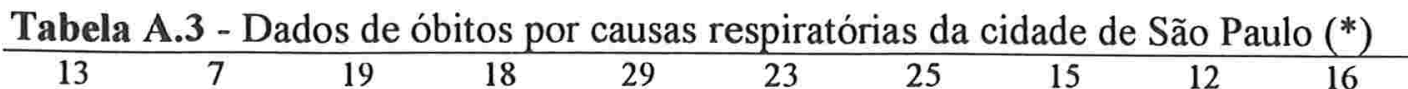

\begin{tabular}{|c|c|c|c|c|c|c|c|c|c|}
\hline 11 & 11 & 11 & 26 & 23 & 17 & 39 & 20 & 12 & 16 \\
\hline 15 & 19 & 24 & 19 & 21 & 19 & 27 & 22 & 14 & 12 \\
\hline 13 & 17 & 19 & 13 & 25 & 23 & 26 & 15 & 22 & 22 \\
\hline 20 & 15 & 12 & 11 & 17 & 34 & 30 & 12 & 16 & 17 \\
\hline 27 & 7 & 19 & 21 & 28 & 26 & 28 & 20 & 16 & 11 \\
\hline 8 & 14 & 19 & 22 & 22 & 38 & 20 & 23 & 12 & 17 \\
\hline 13 & 18 & 13 & 14 & 23 & 24 & 28 & 20 & 13 & 11 \\
\hline 12 & 9 & 22 & 16 & 27 & 32 & 23 & 19 & 12 & 16 \\
\hline 17 & 18 & 19 & 24 & 25 & 30 & 24 & 12 & 12 & 16 \\
\hline 19 & 13 & 17 & 19 & 20 & 25 & 18 & 16 & 19 & 16 \\
\hline 27 & 10 & 24 & 15 & 26 & 30 & 31 & 22 & 19 & 11 \\
\hline 9 & 14 & 23 & 17 & 26 & 20 & 22 & 13 & 17 & 13 \\
\hline 11 & 14 & 15 & 16 & 30 & 27 & 20 & 13 & 19 & 16 \\
\hline 22 & 24 & 15 & 27 & 27 & 32 & 19 & 24 & 17 & 10 \\
\hline 16 & 19 & 17 & 18 & 25 & 22 & 24 & 21 & 20 & 22 \\
\hline 16 & 20 & 19 & 14 & 28 & 21 & 20 & 22 & 17 & 15 \\
\hline 17 & 16 & 19 & 22 & 27 & 26 & 20 & 19 & 17 & 10 \\
\hline 13 & 26 & 14 & 25 & 11 & 35 & 27 & 9 & 18 & 16 \\
\hline 23 & 15 & 15 & 15 & 21 & 29 & 25 & 11 & 12 & 19 \\
\hline 17 & 15 & 14 & 17 & 25 & 32 & 21 & 20 & 19 & 15 \\
\hline 18 & 9 & 20 & 24 & 20 & 28 & 21 & 9 & 14 & 17 \\
\hline 16 & 19 & 14 & 14 & 27 & 36 & 31 & 13 & 16 & 21 \\
\hline 9 & 20 & 16 & 15 & 32 & 33 & 25 & 19 & 21 & 16 \\
\hline 21 & 13 & 21 & 26 & 18 & 50 & 23 & 12 & 8 & 18 \\
\hline 23 & 14 & 22 & 15 & 23 & 37 & 24 & 16 & 11 & 9 \\
\hline 13 & 11 & 25 & 20 & 27 & 28 & 23 & 15 & 14 & 17 \\
\hline 17 & 23 & 18 & 17 & 32 & 24 & 26 & 15 & 7 & 12 \\
\hline 17 & 11 & 14 & 20 & 19 & 33 & 16 & 8 & 16 & 13 \\
\hline 12 & 16 & 14 & 25 & 19 & 22 & 22 & 18 & 12 & 19 \\
\hline 22 & 15 & 10 & 12 & 28 & 37 & 27 & 10 & 12 & 13 \\
\hline 16 & 14 & 16 & 23 & 25 & 26 & 23 & 12 & 21 & 15 \\
\hline 18 & 17 & 17 & 18 & 23 & 25 & 26 & 16 & 17 & 12 \\
\hline 18 & 19 & 13 & 17 & 41 & 38 & 16 & 14 & 16 & 14 \\
\hline 11 & 12 & 21 & 21 & 28 & 25 & 16 & 21 & 15 & 10 \\
\hline 17 & 18 & 18 & 22 & 16 & 25 & 23 & 12 & 10 & 16 \\
\hline 13 & 14 & 21 & 31 & 28 & 15 & 21 & 17 & 19 & 13 \\
\hline
\end{tabular}

(*) ler por colunas 
Tabela A.4 - Dados de óbitos por causas respiratórias da cidade de São Paulo (continuação)

\begin{tabular}{|c|c|c|c|c|c|c|c|c|c|}
\hline 15 & 21 & 12 & 11 & 22 & 13 & 25 & 14 & 9 & 8 \\
\hline 13 & 23 & 14 & 17 & 29 & 22 & 14 & 11 & 18 & 10 \\
\hline 15 & 11 & 16 & 13 & 25 & 23 & 25 & 11 & 22 & 12 \\
\hline 16 & 14 & 15 & 20 & 25 & 25 & 11 & 19 & 18 & 13 \\
\hline 15 & 16 & 16 & 20 & 20 & 23 & 11 & 15 & 13 & 17 \\
\hline 13 & 17 & 11 & 10 & 34 & 30 & 27 & 12 & 9 & 12 \\
\hline 11 & 8 & 11 & 23 & 38 & 24 & 18 & 18 & 13 & 15 \\
\hline 11 & 14 & 15 & 19 & 31 & 21 & 23 & 17 & 19 & 17 \\
\hline 13 & 18 & 11 & 20 & 30 & 22 & 18 & 24 & 15 & 18 \\
\hline 15 & 13 & 16 & 23 & 38 & 19 & 21 & 20 & 16 & 21 \\
\hline 13 & 21 & 12 & 16 & 39 & 18 & 23 & 25 & 19 & 15 \\
\hline 17 & 18 & 16 & 13 & 43 & 19 & 21 & 20 & 17 & 14 \\
\hline 10 & 14 & 17 & 7 & 34 & 21 & 12 & 10 & 16 & 13 \\
\hline 18 & 12 & 14 & 19 & 38 & 25 & 22 & 13 & 14 & 11 \\
\hline 15 & 17 & 14 & 19 & 32 & 18 & 17 & 23 & 15 & 15 \\
\hline 21 & 15 & 11 & 21 & 27 & 16 & 15 & 15 & 20 & 15 \\
\hline 6 & 15 & 18 & 14 & 31 & 13 & 15 & 15 & 20 & 14 \\
\hline 13 & 9 & 14 & 23 & 39 & 21 & 21 & 17 & 17 & 25 \\
\hline 17 & 16 & 10 & 16 & 28 & 17 & 18 & 9 & 19 & 21 \\
\hline 14 & 16 & 11 & 9 & 31 & 19 & 21 & 17 & 13 & 16 \\
\hline 12 & 23 & 16 & 17 & 34 & 17 & 16 & 27 & 14 & 16 \\
\hline 14 & 13 & 14 & 10 & 28 & 20 & 18 & 10 & 13 & 17 \\
\hline 15 & 15 & 13 & 22 & 34 & 16 & 20 & 18 & 16 & 19 \\
\hline 9 & 20 & 21 & 17 & 31 & 21 & 24 & 15 & 14 & 24 \\
\hline 14 & 20 & 14 & 17 & 32 & 23 & 14 & 22 & 14 & 32 \\
\hline 13 & 13 & 9 & 19 & 28 & 23 & 27 & 14 & 16 & 26 \\
\hline 15 & 14 & 20 & 20 & 20 & 21 & 19 & 16 & 16 & 26 \\
\hline 12 & 16 & 16 & 25 & 32 & 18 & 13 & 9 & 14 & 22 \\
\hline 13 & 16 & 18 & 13 & 30 & 20 & 17 & 17 & 22 & \\
\hline 10 & 17 & 14 & 21 & 22 & 17 & 18 & 11 & 14 & \\
\hline 15 & 11 & 17 & 15 & 19 & 17 & 16 & 14 & 22 & \\
\hline 10 & 12 & 18 & 22 & 29 & 18 & 13 & 13 & 21 & \\
\hline 15 & 15 & 19 & 23 & 24 & 22 & 15 & 14 & 11 & \\
\hline 15 & 26 & 18 & 22 & 27 & 19 & 20 & 16 & 19 & \\
\hline 22 & 19 & 14 & 17 & 30 & 25 & 20 & 17 & 12 & \\
\hline 13 & 12 & 14 & 29 & 41 & 20 & 16 & 10 & 20 & \\
\hline 14 & 19 & 20 & 24 & 14 & 20 & 25 & 19 & 21 & \\
\hline
\end{tabular}

$\left({ }^{*}\right)$ ler por colunas 
Tabela A.5 - Dados de óbitos da cidade de São Paulo (*)

\begin{tabular}{llllllllll}
\hline 133 & 128 & 142 & 150 & 183 & 163 & 167 & 142 & 123 & 140 \\
125 & 137 & 135 & 162 & 182 & 188 & 158 & 156 & 123 & 151 \\
135 & 157 & 137 & 171 & 162 & 158 & 171 & 150 & 113 & 158 \\
148 & 144 & 172 & 168 & 145 & 165 & 181 & 189 & 154 & 139 \\
140 & 143 & 149 & 171 & 159 & 183 & 189 & 143 & 142 & 152 \\
167 & 134 & 161 & 159 & 165 & 184 & 185 & 135 & 150 & 127 \\
120 & 146 & 150 & 148 & 187 & 176 & 175 & 168 & 151 & 150 \\
131 & 143 & 162 & 146 & 175 & 189 & 203 & 163 & 140 & 158 \\
118 & 144 & 178 & 145 & 163 & 192 & 193 & 147 & 143 & 161 \\
110 & 144 & 138 & 170 & 196 & 162 & 186 & 144 & 150 & 135 \\
165 & 136 & 158 & 155 & 166 & 184 & 180 & 162 & 139 & 130 \\
161 & 139 & 160 & 155 & 181 & 203 & 191 & 142 & 116 & 135 \\
136 & 147 & 158 & 156 & 154 & 192 & 159 & 143 & 126 & 145 \\
109 & 123 & 133 & 149 & 189 & 174 & 161 & 132 & 143 & 142 \\
153 & 155 & 136 & 152 & 177 & 195 & 180 & 130 & 137 & 137 \\
168 & 143 & 164 & 156 & 181 & 185 & 159 & 138 & 139 & 137 \\
141 & 135 & 154 & 154 & 189 & 206 & 180 & 154 & 151 & 144 \\
150 & 132 & 158 & 148 & 198 & 190 & 195 & 140 & 136 & 163 \\
133 & 173 & 143 & 175 & 164 & 205 & 149 & 127 & 143 & 136 \\
146 & 151 & 164 & 140 & 172 & 187 & 160 & 150 & 145 & 154 \\
145 & 128 & 135 & 135 & 185 & 204 & 153 & 126 & 131 & 151 \\
132 & 122 & 133 & 173 & 174 & 195 & 148 & 135 & 132 & 144 \\
152 & 173 & 131 & 142 & 179 & 216 & 157 & 110 & 142 & 161 \\
135 & 174 & 139 & 164 & 177 & 201 & 156 & 153 & 142 & 114 \\
156 & 136 & 161 & 164 & 171 & 228 & 170 & 159 & 138 & 135 \\
151 & 151 & 142 & 146 & 185 & 200 & 148 & 160 & 114 & 142 \\
123 & 138 & 153 & 145 & 183 & 160 & 155 & 155 & 149 & 154 \\
127 & 167 & 136 & 154 & 178 & 200 & 147 & 136 & 108 & 141 \\
141 & 120 & 144 & 156 & 156 & 192 & 135 & 120 & 136 & 146 \\
149 & 122 & 141 & 180 & 188 & 174 & 140 & 127 & 126 & 162 \\
147 & 147 & 143 & 147 & 190 & 170 & 144 & 129 & 133 & 141 \\
147 & 128 & 144 & 143 & 172 & 191 & 144 & 145 & 154 & 143 \\
133 & 134 & 120 & 166 & 176 & 192 & 157 & 155 & 142 & 106 \\
141 & 144 & 143 & 139 & 192 & 203 & 169 & 162 & 150 & 121 \\
135 & 141 & 137 & 154 & 182 & 166 & 161 & 152 & 148 & 144 \\
150 & 152 & 140 & 186 & 192 & 200 & 167 & 128 & 122 & 147 \\
\hline$* 11$ & 142 & 157 & 190 & 177 & 168 & 167 & 134 & 141 & 134 \\
\hline 1459 & & & & & & & &
\end{tabular}


Tabela A.6 - Dados de óbitos da cidade de São Paulo (continuação)

\begin{tabular}{|c|c|c|c|c|c|c|c|c|c|}
\hline 135 & 146 & 133 & 156 & 183 & 145 & 180 & 148 & 125 & 121 \\
\hline 137 & 144 & 120 & 119 & 214 & 148 & 156 & 132 & 168 & 120 \\
\hline 137 & 115 & 138 & 157 & 195 & 169 & 186 & 159 & 154 & 156 \\
\hline 129 & 146 & 131 & 156 & 222 & 160 & 155 & 144 & 158 & 151 \\
\hline 138 & 152 & 125 & 149 & 177 & 151 & 163 & 151 & 155 & 150 \\
\hline 146 & 143 & 132 & 140 & 203 & 139 & 166 & 158 & 132 & 136 \\
\hline 135 & 132 & 127 & 173 & 200 & 181 & 146 & 141 & 146 & 141 \\
\hline 130 & 155 & 137 & 148 & 184 & 166 & 142 & 138 & 126 & 160 \\
\hline 147 & 137 & 99 & 156 & 178 & 155 & 139 & 145 & 121 & 141 \\
\hline 129 & 145 & 164 & 165 & 212 & 156 & 151 & 155 & 137 & 141 \\
\hline 166 & 120 & 123 & 138 & 229 & 162 & 162 & 155 & 153 & 144 \\
\hline 150 & 151 & 146 & 143 & 229 & 144 & 172 & 147 & 165 & 128 \\
\hline 130 & 130 & 151 & 130 & 210 & 154 & 155 & 149 & 154 & 149 \\
\hline 115 & 132 & 151 & 153 & 195 & 174 & 172 & 138 & 176 & 135 \\
\hline 162 & 157 & 151 & 152 & 207 & 160 & 166 & 193 & 144 & 137 \\
\hline 166 & 140 & 128 & 144 & 196 & 149 & 148 & 147 & 154 & 128 \\
\hline 140 & 132 & 158 & 142 & 184 & 154 & 135 & 144 & 157 & 148 \\
\hline 134 & 122 & 131 & 178 & 193 & 171 & 162 & 151 & 135 & 179 \\
\hline 137 & 129 & 151 & 138 & 183 & 182 & 152 & 136 & 158 & 190 \\
\hline 126 & 132 & 123 & 137 & 183 & 163 & 142 & 158 & 136 & 173 \\
\hline 124 & 147 & 175 & 126 & 194 & 147 & 145 & 159 & 138 & 198 \\
\hline 114 & 120 & 151 & 147 & 212 & 178 & 172 & 153 & 139 & 176 \\
\hline 156 & 144 & 152 & 147 & 169 & 150 & 146 & 148 & 131 & 151 \\
\hline 125 & 148 & 163 & 150 & 179 & 157 & 134 & 137 & 138 & 174 \\
\hline 121 & 164 & 160 & 150 & 164 & 178 & 147 & 166 & 151 & 190 \\
\hline 129 & 160 & 153 & 167 & 181 & 147 & 184 & 129 & 133 & 145 \\
\hline 146 & 160 & 138 & 149 & 153 & 154 & 136 & $1+3$ & 175 & 176 \\
\hline 136 & 138 & 135 & 164 & 185 & 153 & 107 & 150 & 128 & 164 \\
\hline 160 & 140 & 145 & 139 & 192 & 155 & 161 & 165 & 142 & \\
\hline 126 & 133 & 158 & 151 & 161 & 154 & 154 & 149 & 152 & \\
\hline 138 & 136 & 138 & 168 & 184 & 150 & 159 & 144 & 147 & \\
\hline 127 & 125 & 118 & 169 & 183 & 187 & 145 & 122 & 131 & \\
\hline 139 & 120 & 141 & 177 & 167 & 158 & 162 & 153 & 105 & \\
\hline 133 & 160 & 154 & 157 & 170 & 171 & 141 & 129 & 150 & \\
\hline 154 & 141 & 147 & 162 & 183 & 164 & 124 & 155 & 122 & \\
\hline 133 & 125 & 153 & 168 & 186 & 172 & 145 & 135 & 141 & \\
\hline 120 & 151 & 154 & 183 & 153 & 176 & 163 & 154 & 150 & \\
\hline
\end{tabular}

$\left.{ }^{*}\right)$ ler por colunas 
Tabela A.7 - Dados da concentração do agente poluidor PM10 da cidade de São Paulo $(*)$

\begin{tabular}{|c|c|c|c|c|c|c|c|c|c|}
\hline 97,70 & 49,81 & 60,19 & 183,88 & 49,81 & 37,53 & 47,46 & 33,63 & 64,55 & 35,88 \\
\hline 102,94 & 47,85 & 75,85 & 186,25 & 45,94 & 36,18 & 44,63 & 35,32 & 48,67 & 40,90 \\
\hline 76,63 & 95,93 & 63,60 & 149,88 & 45,44 & 44,86 & 50,75 & 29,74 & 58,01 & 49,06 \\
\hline 75,33 & 94,45 & 59,38 & 43,33 & 71,95 & 59,21 & 68,23 & 43,34 & 70,04 & 59,91 \\
\hline 94,65 & 87,85 & 60,59 & 35,18 & 34,68 & 45,30 & 67,83 & 43,79 & 69,54 & 57,02 \\
\hline 145,95 & 106,53 & 66,48 & 53,65 & 44,85 & 65,58 & 63,10 & 51,86 & 58,01 & 48,17 \\
\hline 148,17 & 112,70 & 42,77 & 58,68 & 62,01 & 43,61 & 64,27 & 53,46 & 54,69 & 56,92 \\
\hline 129,41 & 91,83 & 75,99 & 49,37 & 87,34 & 35,14 & 45,70 & 41,77 & 45,95 & 77,74 \\
\hline 114,21 & 26,76 & 80,41 & 52,74 & 84,90 & 58,20 & 48,18 & 54,35 & 30,48 & 51,94 \\
\hline 85,78 & 47,34 & 96,61 & 63,55 & 91,98 & 77,88 & 51,19 & 33,29 & 39,60 & 46,00 \\
\hline 70,05 & 53,94 & 55,94 & 51,55 & 123,27 & 84,66 & 50,99 & 35,84 & 41,73 & 48,74 \\
\hline 115,03 & 75,42 & 105,03 & 53,92 & 64,08 & 86,51 & 41,00 & 39,79 & 48,33 & 39,83 \\
\hline 110,31 & 49,58 & 151,42 & 74,03 & 54,57 & 81,40 & 40,14 & 53,63 & 45,82 & 39,62 \\
\hline 55,53 & 90,66 & 104,94 & 105,36 & 37,89 & 52,10 & 56,18 & 54,67 & 44,98 & 48,09 \\
\hline 54,99 & 80,88 & 53,18 & 121,28 & 42,69 & 43,76 & 75,32 & 43,89 & 55,55 & 52,97 \\
\hline 66,14 & 39,64 & 51,89 & 122,39 & 46,64 & 39,31 & 68,19 & 36,53 & 55,66 & 54,86 \\
\hline 71,90 & 60,02 & 81,05 & 117,85 & 46,37 & 49,67 & 66,19 & 39,01 & 50,61 & 52,24 \\
\hline 51,91 & 81,72 & 93,02 & 95,94 & 49,81 & 65,12 & 53,11 & 31,41 & 53,90 & 46,97 \\
\hline 73,44 & 100,61 & 161,67 & 81,81 & 50,99 & 74,16 & 47,06 & 39,82 & 58,55 & 53,79 \\
\hline 106,21 & 95,53 & 158,25 & 126,33 & 63,78 & 74,42 & 43,41 & 43,21 & 48,41 & 48,84 \\
\hline 139,41 & 103,32 & 42,58 & 143,05 & 48,08 & 71,06 & 56,35 & 43,25 & 39,66 & 45,58 \\
\hline 120,63 & 101,07 & 41,89 & 52,64 & 67,79 & 52,21 & 54,30 & 40,88 & 36,59 & 44,42 \\
\hline 71,82 & 68,93 & 46,36 & 43,15 & 81,08 & 41,30 & 44,81 & 42,23 & 37,51 & 38,74 \\
\hline 54,83 & 88,46 & 95,72 & 60,24 & 62,15 & 69,95 & 67,07 & 34,80 & 49,67 & 38,48 \\
\hline 71,13 & 107,60 & 125,23 & 75,74 & 43,01 & 60,73 & 59,43 & 30,97 & 50,58 & 52,69 \\
\hline 96,27 & 98,40 & 120,81 & 47,60 & 44,37 & 54,76 & 43,27 & 42,83 & 52,92 & 81,09 \\
\hline 115,17 & 52,30 & 91,79 & 68,10 & 51,02 & 43,81 & 28,62 & 46,90 & 55,34 & 83,68 \\
\hline 102,80 & 76,22 & 68,66 & 66,47 & 68,75 & 33,09 & 46,39 & 56,24 & 61,20 & 65,32 \\
\hline 43,39 & 106,38 & 40,42 & 61,08 & 58,30 & 26,76 & 45,13 & 58,78 & 55,06 & 87,10 \\
\hline 53,43 & 112,78 & 52,08 & 76,49 & 65,76 & 48,54 & 54,68 & 40,90 & 51,71 & 83,19 \\
\hline 68,01 & 74,97 & 53,99 & 45,49 & 50,93 & 46,36 & 58,38 & 31,63 & 49,88 & 92,51 \\
\hline 68,39 & 131,10 & 83,86 & 40,02 & 44,37 & 46,10 & 52,89 & 28,42 & 68,64 & 64,57 \\
\hline 72,90 & 147,97 & 95,65 & 40,76 & 41,52 & 37,51 & 44,25 & 42,49 & 78,97 & 55,98 \\
\hline 72,33 & 72,35 & 77,50 & 44,05 & 49,18 & 40,96 & 27,88 & 61,36 & 84,01 & 50,36 \\
\hline 70,70 & 48,17 & 93,54 & 27,39 & 72,06 & 49,36 & 40,68 & 68,11 & 67,74 & 34,51 \\
\hline 50,23 & 35,33 & 137,87 & 33,85 & 82,58 & 55,11 & 41,57 & 73,78 & 48,78 & 25,52 \\
\hline 41,71 & 34,04 & 158,79 & 39,42 & 71,66 & 46.36 & 36,84 & 79,42 & 37,09 & 43,59 \\
\hline
\end{tabular}

$\left({ }^{*}\right)$ ler por colunas 
Tabela A.8 - Dados da concentração do agente poluidor PM10 da cidade de São Paulo (continuação)

\begin{tabular}{ccccccc}
\hline 43,61 & 30,14 & 60,57 & 91,81 & 50,92 & 73,36 & 52,26 \\
66,26 & 40,35 & 88,02 & 86,09 & 58,82 & 43,22 & 40,95 \\
86,66 & 36,70 & 75,88 & 84,43 & 49,92 & 53,00 & 53,83 \\
54,76 & 55,65 & 90,04 & 111,23 & 26,59 & 69,32 & 61,69 \\
56,45 & 94,48 & 123,75 & 121,73 & 31,23 & 81,59 & 65,24 \\
59,22 & 76,79 & 102,71 & 123,31 & 51,68 & 51,60 & 64,92 \\
80,29 & 50,12 & 69,53 & 99,50 & 65,75 & 61,69 & 46,22 \\
94,72 & 49,44 & 64,48 & 97,83 & 63,89 & 63,13 & 48,57 \\
77,62 & 40,00 & 105,81 & 102,02 & 30,82 & 42,50 & 58,28 \\
99,10 & 39,09 & 147,41 & 95,11 & 31,64 & 51,65 & 51,66 \\
94,35 & 70,49 & 141,65 & 92,10 & 43,71 & 37,53 & 59,87 \\
47,82 & 93,60 & 129,64 & 52,44 & 50,60 & 47,74 & 67,46 \\
49,20 & 100,32 & 66,74 & 29,45 & 42,96 & 67,96 & 47,41 \\
67,93 & 62,30 & 74,96 & 40,44 & 50,02 & 63,31 & 32,59 \\
75,64 & 98,21 & 97,18 & 52,88 & 38,52 & 43,65 & 35,63 \\
82,64 & 51,82 & 59,06 & 77,11 & 31,63 & 50,56 & 49,99 \\
86,51 & 26,65 & 57,24 & 95,22 & 53,43 & 99,13 & 35,43 \\
66,24 & 39,22 & 56,50 & 107,66 & 60,81 & 98,23 & 44,78 \\
61,25 & 66,67 & 46,96 & 110,96 & 47,04 & 72,94 & 45,60 \\
83,83 & 78,97 & 51,33 & 90,65 & 33,33 & 79,68 & 41,56 \\
86,79 & 73,34 & 53,97 & 108,55 & 36,52 & 48,87 & 37,01 \\
52,83 & 61,89 & 82,36 & 120,76 & 64,08 & 26,88 & 52,24 \\
58,89 & 61,95 & 132,24 & 132,79 & 91,94 & 37,92 & 50,73 \\
75,81 & 52,48 & 145,89 & 152,98 & 63,47 & 50,93 & 54,31 \\
72,28 & 66,56 & 121,79 & 97,19 & 73,38 & 37,67 & 40,71 \\
68,51 & 68,87 & 103,95 & 45,96 & 74,88 & 32,05 & 46,51 \\
89,02 & 104,53 & 147,26 & 59,14 & 60,33 & 43,32 & 38,00 \\
97,53 & 84,06 & 148,15 & 103,65 & 74,83 & 54,37 & 32,87 \\
83,65 & 68,88 & 106,49 & 150,49 & 51,41 & 47,94 & 37,89 \\
57,40 & 48,42 & 59,16 & 50,49 & 39,62 & 46,67 & 52,08 \\
46,01 & 37,82 & 26,88 & 34,41 & 33,38 & 40,37 & 40,81 \\
40,83 & 36,31 & 49,60 & 70,96 & 39,52 & 38,90 & \\
27,58 & 48,63 & 72,23 & 113,72 & 35,56 & 42,87 & \\
57,06 & 73,09 & 30,16 & 49,66 & 48,20 & 45,47 & \\
62,07 & 76,18 & 26,22 & 31,08 & 40,56 & 37,08 & \\
56,92 & 77,47 & 32,48 & 49,36 & 34,54 & 43,87 & \\
26,07 & 49,07 & 55,69 & 57,11 & 53,41 & 39,69 & \\
\hline 795 & 00195 & & & & & \\
\hline
\end{tabular}

$(*)$ ler por colunas 
APÊNDICE B

PROGRAMAS 
B.1 Programa referente ao teste de linearidade de McLeod e Li (S-plus)

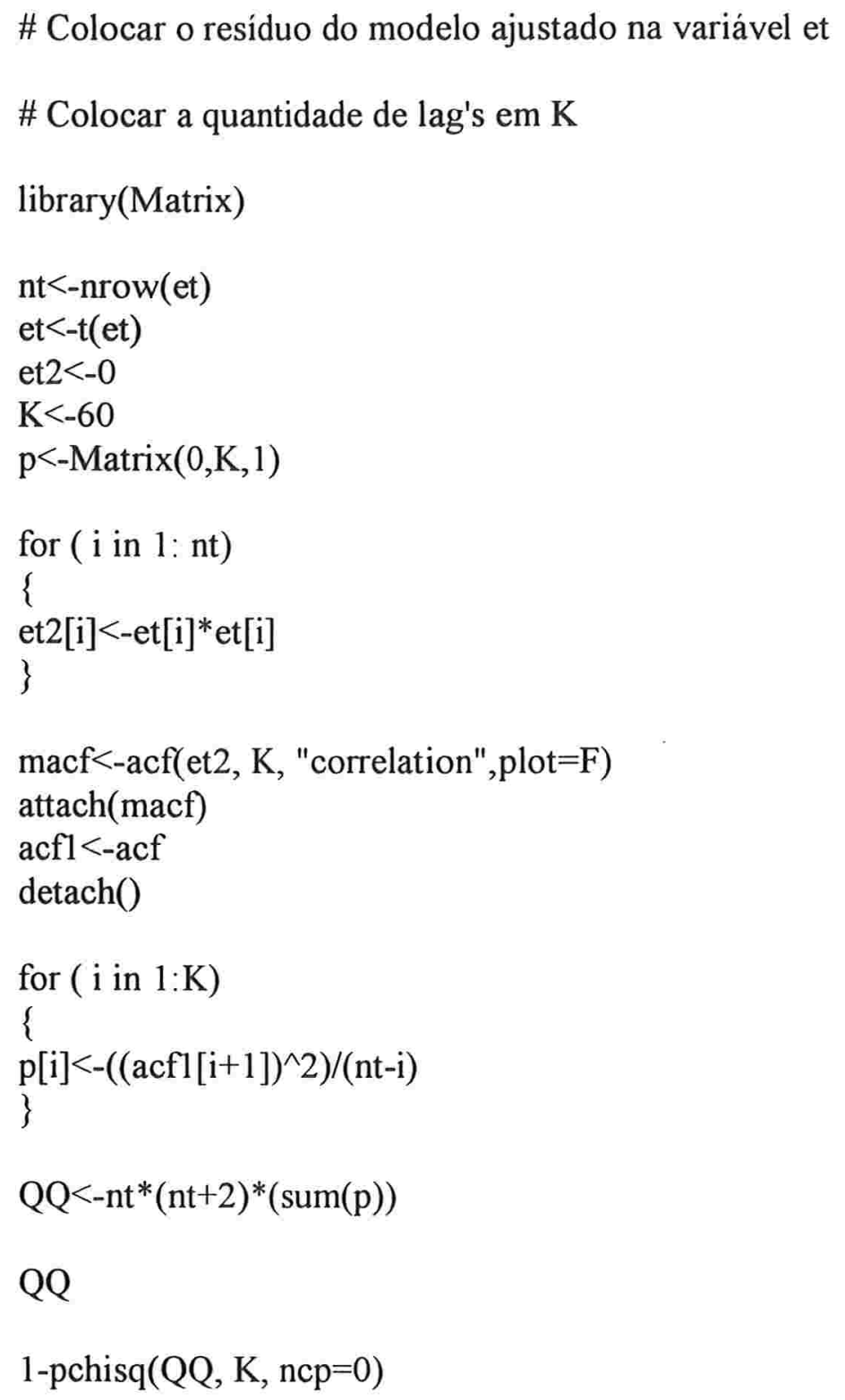




\section{B.2 Programa referente ao teste de linearidade de Tsay (S-plus)}

\# Colocar a ordem do modelo auto-regressivo ajustado na variável m

\# As séries originais e com as respectivas defasagens foram colocadas no arquivo \# de entrada DADOS

\# Série RESP

$m<-3$

$\mathrm{e} 1<-0$

$\mathrm{e} 2<-0$

e $3<-0$

e $21<-0$

e22<-0

e23<-0

$\mathrm{e} 24<-0$

$\mathrm{e} 25<-0$

e26<-0

$\mathrm{ff}<-0$

ffl $<-0$

fit $1<-\operatorname{lm}$ (formula $=$ RESP $\sim$ RESP1 + RESP2 + RESP3, data $=$ DADOS, na. action $=$ na.omit)

e1<-resid(fit1)

fit $2<-$ manova $($ formula $=$

cbind(RESP $1 *$ RESP1,RESP2*RESP1,RESP3*RESP1,RESP2*RESP2,RESP3*R ESP2,RESP3 $*$ RESP3) RESP1 + RESP2+RESP3, data = DADOS, na.action=na.omit)

e2<-resid(fit2)

$\mathrm{n}<-\operatorname{nrow}(\mathrm{e} 2)$

$\mathrm{r}<-\operatorname{ncol}(\mathrm{e} 2)$

e21<-resid(fit2)[,1]

e22<- resid(fit2)[,2]

e23<- resid(fit2)[,3]

e24<- resid(fit2)[,4]

e25<- resid(fit2)[,5]

e26<- resid(fit2)[,6] 


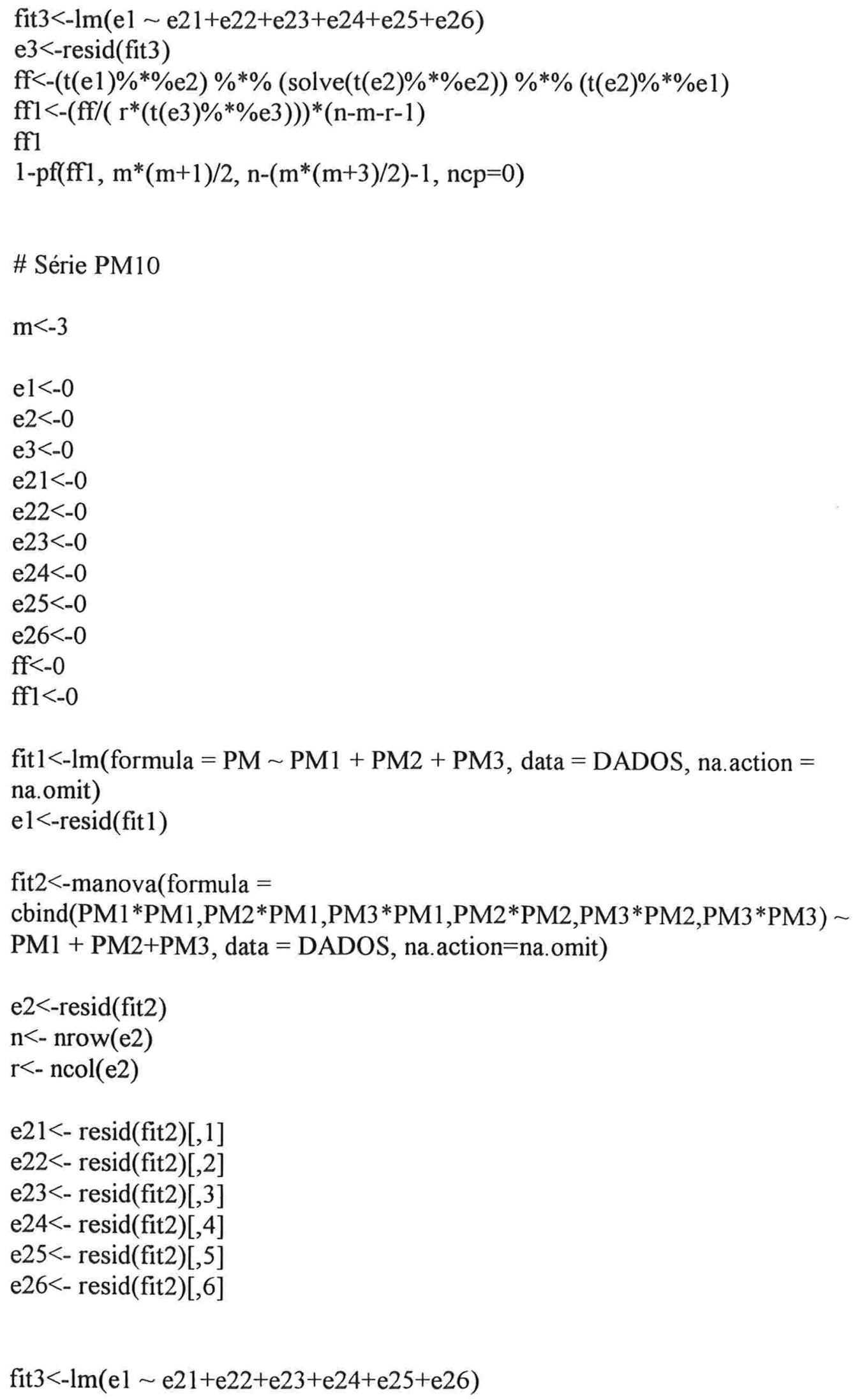


e3<-resid(fit3)

ff<- $(\mathrm{t}(\mathrm{e} 1) \% * \% \mathrm{e} 2) \% * \%$ (solve $(\mathrm{t}(\mathrm{e} 2) \% * \% \mathrm{e} 2)) \% * \%(\mathrm{t}(\mathrm{e} 2) \% * \% \mathrm{e} 1)$

ff $1<-\left(\mathrm{ff} /\left(\mathrm{r}^{*}(\mathrm{t}(\mathrm{e} 3) \% * \% \mathrm{e} 3)\right)\right) *(\mathrm{n}-\mathrm{m}-\mathrm{r}-1)$

ff1

$1-p f\left(f f 1, m^{*}(m+1) / 2, n-(m *(m+3) / 2)-1, n c p=0\right)$

\# Série TEMP

$m<-3$

$\mathrm{e} 1<-0$

$\mathrm{e} 2<-0$

e $3<-0$

e2 $1<-0$

e $22<-0$

e23<-0

e24<-0

$\mathrm{e} 25<-0$

e26<-0

ff $<-0$

ff $1<-0$

fit $1<-\operatorname{lm}$ (formula $=$ TEMP $\sim$ TEMP1 + TEMP2 + TEMP3, data = DADOS, na.action $=$ na.omit $)$

e1<-resid(fit1)

fit $2<$-manova(formula $=$

cbind(TEMP1*TEMP1,TEMP2*TEMP1,TEMP3*TEMP1,TEMP2*TEMP2, TEM P3*TEMP2,TEMP3*TEMP3) TEMP1 + TEMP2+TEMP3, data = DADOS, na. action=na.omit)

e2<-resid(fit2)

$\mathrm{n}<-\operatorname{nrow}(\mathrm{e} 2)$

$\mathrm{r}<-\operatorname{ncol}(\mathrm{e} 2)$

e21<- resid(fit 2$)[, 1]$

e22<- resid(fit2)[,2]

e23<- resid(fit2)[,3]

e24<- resid(fit2)[,4]

e25<- resid(fit2)[,5]

e26<- resid(fit 2$)[, 6]$ 
fit $3<-\operatorname{lm}(\mathrm{e} 1 \sim \mathrm{e} 21+\mathrm{e} 22+\mathrm{e} 23+\mathrm{e} 24+\mathrm{e} 25+\mathrm{e} 26)$

e3<-resid(fit3)

ff<-(t(e1)\%*\%e2) \%*\% (solve(t(e2)\%*\%e2)) \%*\%(t(e2)\%*\%el)

ffl $<-(f f /(r *(t(e 3) \% * \% e 3))) *(n-m-r-1)$

ff1

$1-p f\left(f f 1, m^{*}(m+1) / 2, n-(m *(m+3) / 2)-1, n c p=0\right)$

\# Série TOT

$m<-3$

e $1<-0$

e $2<-0$

e $3<-0$

e $21<-0$

e $22<-0$

e $23<-0$

e24<-0

e $25<-0$

e26<-0

ff $<-0$

ff $1<-0$

fit $1<-\operatorname{lm}($ formula $=$ TOT $\sim$ TOT $1+$ TOT $2+$ TOT 3, data $=$ DADOS, na.action $=$ na.omit)

e1<-resid(fit 1)

fit $2<-$ manova $($ formula $=$

cbind(TOT1*TOT1,TOT2*TOT1,TOT3*TOT1,TOT2*TOT2,TOT3*TOT2,TOT3

*TOT3) $\sim$ TOT1 + TOT2+TOT3, data $=$ DADOS, na.action=na.omit)

e2<-resid(fit2)

$\mathrm{n}<-\operatorname{nrow}(\mathrm{e} 2)$

$\mathrm{r}<-\mathrm{ncol}(\mathrm{e} 2)$

e21<- resid(fit2)[,1]

e22<- resid(fit2)[,2]

e23<- resid(fit2)[,3]

e24<- resid(fit2)[,4]

e25<- resid(fit2)[,5]

e26<- resid(fit2)[,6]

fit $3<-\operatorname{lm}(\mathrm{e} 1 \sim \mathrm{e} 21+\mathrm{e} 22+\mathrm{e} 23+\mathrm{e} 24+\mathrm{e} 25+\mathrm{e} 26)$

e3<-resid(fit3) 
ff<-(t(e1)\%*\%e2) \%*\% (solve(t(e2)\%*\%e2)) \%*\%(t(e2)\%*\%el)

$\mathrm{ffl}<-\left(\mathrm{ff} /\left(\mathrm{r}^{*}(\mathrm{t}(\mathrm{e} 3) \% * \% \mathrm{e} 3)\right)\right) *(n-m-r-1)$

ff1

$1-p f\left(f f 1, m^{*}(m+1) / 2, n-\left(m^{*}(m+3) / 2\right)-1, n c p=0\right)$ 


\section{B.3 Programa referente ao teste de linearidade de Poggi e Portier (S-plus)}

\# Programa elaborado para $\mathrm{p}=3$

\# As funções F,H e K são calculadas nos elementos de q

\# As séries originais e com as respectivas defasagens foram colocadas no arquivo \# de entrada DADOS. A série PM10 com as respectivas defasagens foram \# colocadas no arquivo de entrada DPM10

\# Vetores iniciais para a serie TEMP

$\mathrm{y}<-\mathrm{DADOS}[, 1]$

$\mathrm{x}<-\mathrm{DADOS}[, 2]$

$\mathrm{x} 1<-\mathrm{DADOS}[, 3]$

$\mathrm{x} 2<-\mathrm{DADOS}[, 4]$

\# Vetores iniciais para a serie RESP

$\mathrm{y}<-\mathrm{DADOS}[, 5]$

$\mathrm{x}<$-DADOS[,6]

$\mathrm{x} 1<$-DADOS[,7]

$\mathrm{x} 2<-\mathrm{DADOS}[, 8]$

\# Vetores iniciais para a serie TOT

$\mathrm{y}<-$ DADOS[,9]

$\mathrm{x}<$-DADOS[,10]

$\mathrm{x} 1<-\mathrm{DADOS}[, 11]$

$\mathrm{x} 2<-\mathrm{DADOS}[, 12]$

\# Vetores iniciais para a serie PM10

$\mathrm{y}<-$ DADPM10[,1]

$x<$-DADPM10[,2]

$\mathrm{x} 1<-\mathrm{DADPM} 10[, 3]$

$\mathrm{x} 2<-\mathrm{DADPM} 10 \mathrm{~S}[, 4]$

library(Matrix)

$\mathrm{v}<-0$

$\mathrm{n}<$-length(x)

sigma $<-\operatorname{sqrt}(\operatorname{var}(\mathrm{x}))$ 


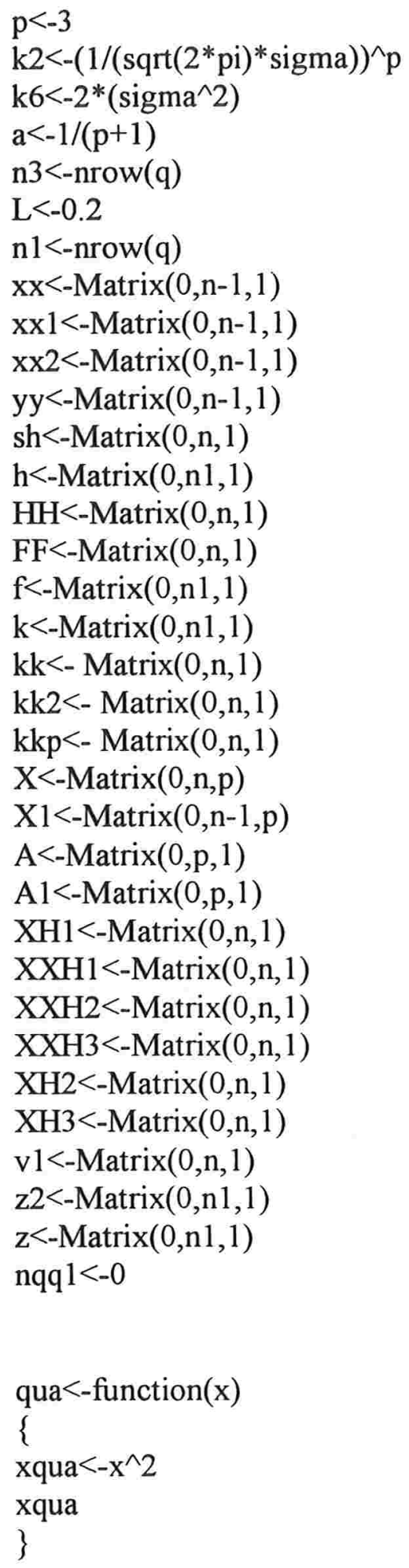




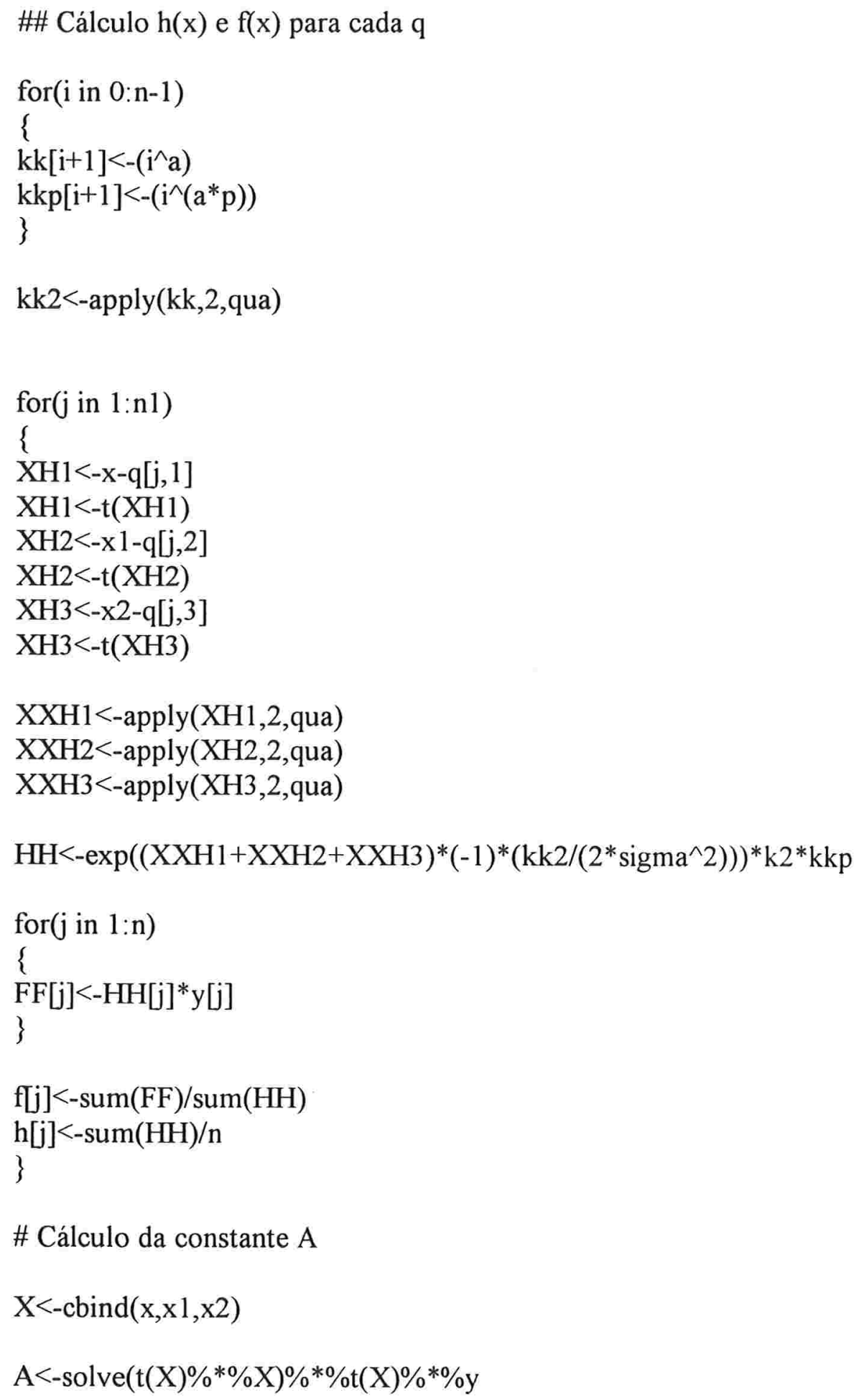




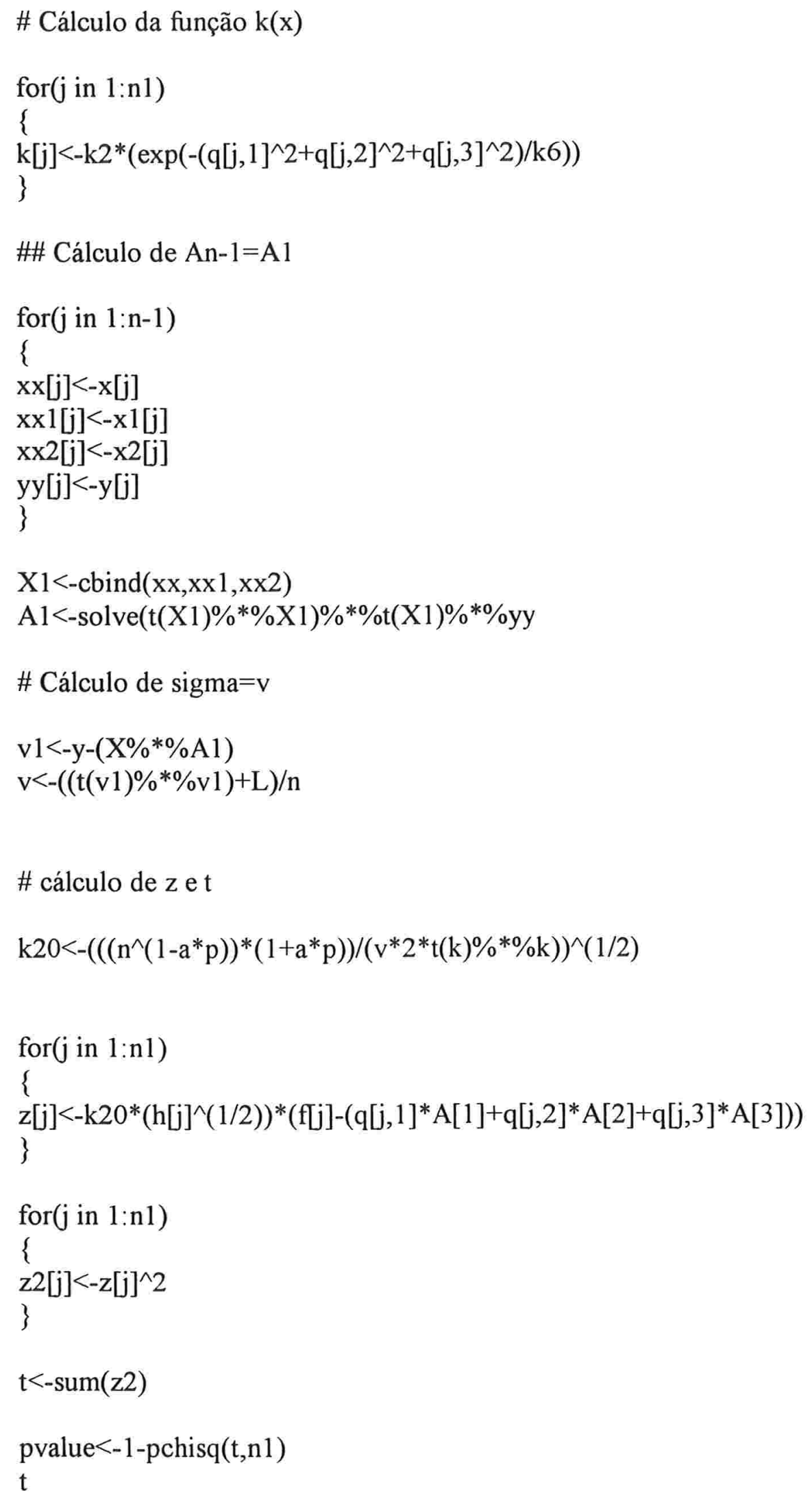




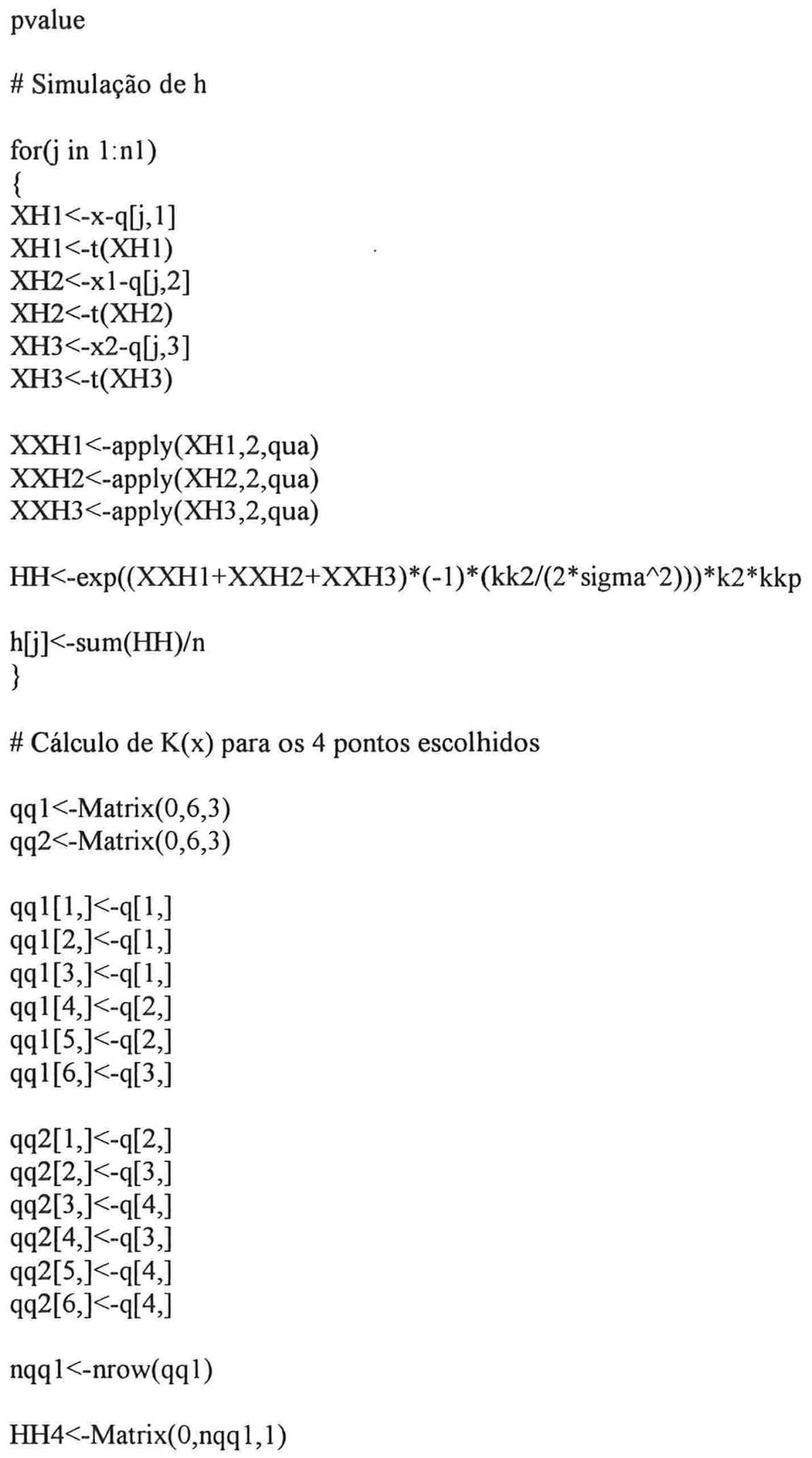


for( $\mathrm{j}$ in 1:nqq1)

\{

$\mathrm{XH} 1<-$ Matrix $(0, \mathrm{n}, 1)$

XH2<-Matrix $(0, \mathrm{n}, 1)$

$\mathrm{XH} 3<-$ Matrix $(0, \mathrm{n}, 1)$

XXH1<-Matrix $(0, \mathrm{n}, 1)$

$\mathrm{XXH} 2<-$ Matrix $(0, \mathrm{n}, 1)$

$\mathrm{XXH} 3<$-Matrix $(0, \mathrm{n}, 1)$

$\mathrm{HH} 1<-$ Matrix $(0, \mathrm{n}, 1)$

$\mathrm{XH} 1<-\mathrm{X}-\mathrm{qq} 1[\mathrm{j}, 1]$

$\mathrm{XH} 1<-\mathrm{t}(\mathrm{XH} 1)$

$\mathrm{XH} 2<-\mathrm{x} 1-\mathrm{qq} 1[\mathrm{j}, 2]$

$\mathrm{XH} 2<-\mathrm{t}(\mathrm{XH} 2)$

$\mathrm{XH} 3<-\mathrm{x} 2-\mathrm{qq} 1[\mathrm{j}, 3]$

XH3<-t(XH3)

XXH1<-apply(XH1,2,qua)

$\mathrm{XXH} 2<$-apply(XH2,2,qua)

XXH3<-apply(XH3,2,qua)

$\mathrm{HH} 1<-\mathrm{k} 2 * \exp \left(\left(-(\mathrm{XXH} 1+\mathrm{XXH} 2+\mathrm{XXH} 3)^{*}\left(\mathrm{kk}^{\wedge} 2\right)\right) / \mathrm{k} 6\right)$

$\mathrm{XH} 1<-\operatorname{Matrix}(0, \mathrm{n}, 1)$

$\mathrm{XH} 2<-$ Matrix $(0, \mathrm{n}, 1)$

XH3<-Matrix $(0, \mathrm{n}, 1)$

$\mathrm{XXH} 1<$-Matrix $(0, \mathrm{n}, 1)$

$\mathrm{XXH} 2<-$ Matrix $(0, \mathrm{n}, 1)$

XXH3<-Matrix $(0, n, 1)$

$\mathrm{HH} 2<-$ Matrix $(0, \mathrm{n}, 1)$

$\mathrm{XH} 1<-\mathrm{x}-\mathrm{qq} 2[\mathrm{j}, 1]$

$\mathrm{XH} 1<-\mathrm{t}(\mathrm{XH} 1)$

$\mathrm{XH} 2<-\mathrm{x} 1-\mathrm{qq} 2[\mathrm{j}, 2]$

$\mathrm{XH} 2<-\mathrm{t}(\mathrm{XH} 2)$

$\mathrm{XH} 3<-\mathrm{x} 2-\mathrm{qq} 2[\mathrm{j}, 3]$

$\mathrm{XH} 3<-\mathrm{t}(\mathrm{XH} 3)$

XXH1<-apply(XH1,2,qua)

XXH2<-apply(XH2,2,qua)

$\mathrm{XXH} 3<-$ apply $(\mathrm{XH} 3,2$, qua $)$

$\mathrm{HH} 2<-\mathrm{k} 2 * \exp \left(\left(-(\mathrm{XXH} 1+\mathrm{XXH} 2+\mathrm{XXH} 3) *\left(\mathrm{kk}^{\wedge} 2\right)\right) / \mathrm{k} 6\right)$ 
$\mathrm{HH} 3<-\mathrm{HH} 1{ }^{*} \mathrm{HH} 2{ }^{*} \mathrm{kk}$

$\mathrm{HH} 4[\mathrm{j}]<-\operatorname{sum}(\mathrm{HH} 3) / \mathrm{n}$

\} 
B.4 Programa referente ao teste de linearidade de Brillinger e Irizarry (Splus)

\# Colocar a série em y

\# Este programa particiona a série original em L séries de tamanho v, para isto \# precisamos colocar o valor do parâmetro $\mathrm{v}$ na variável $\mathrm{v}$

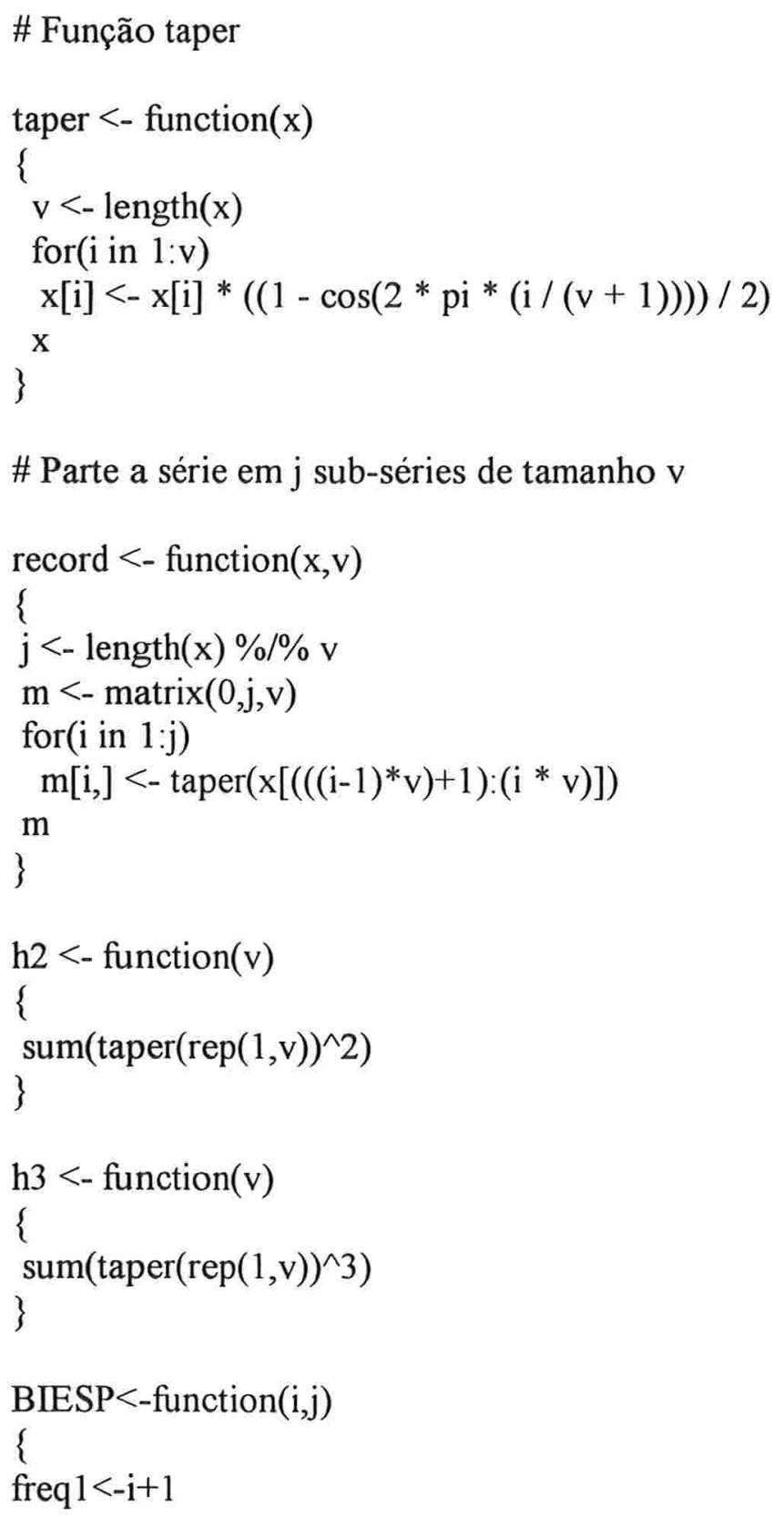




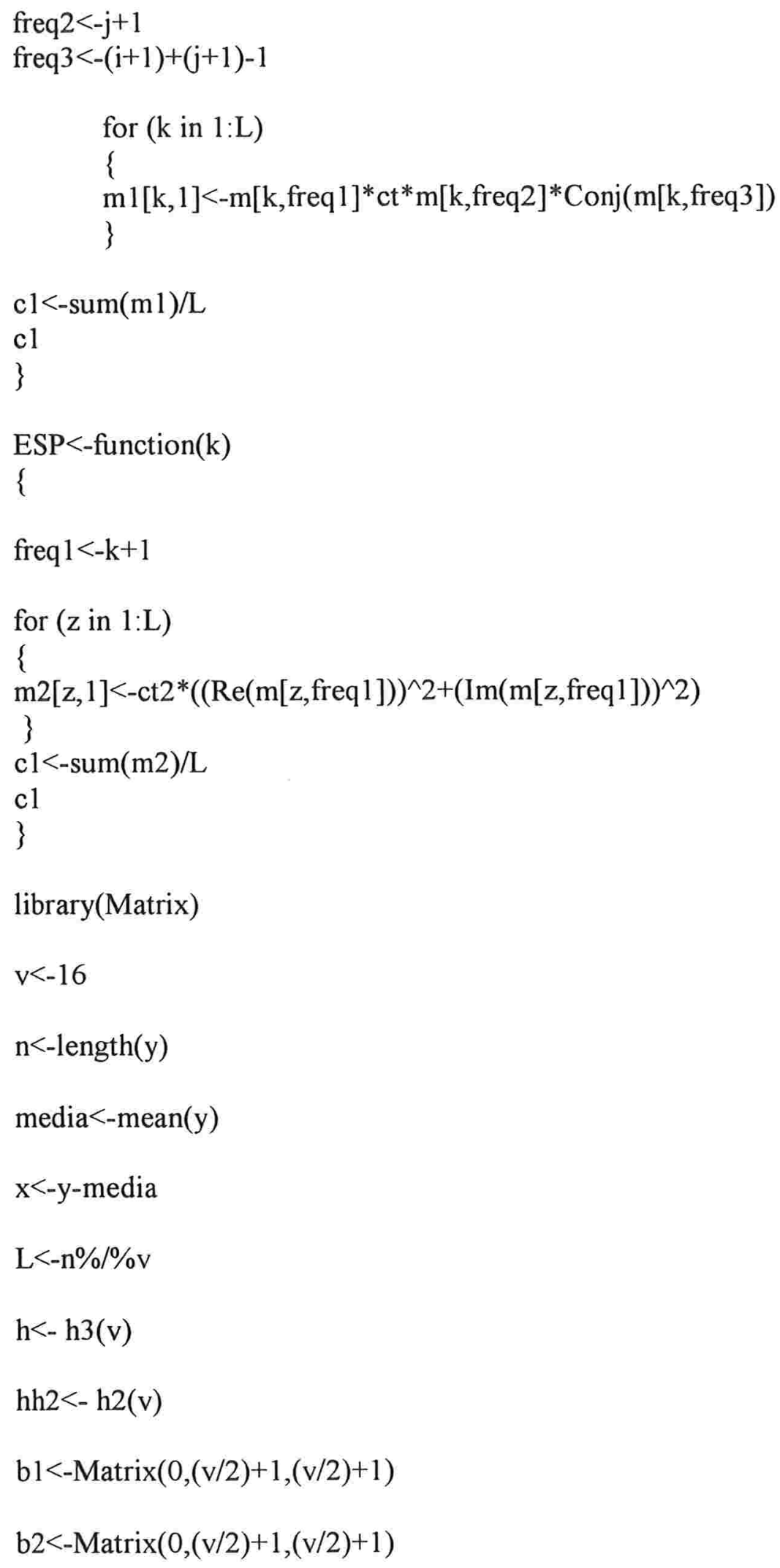




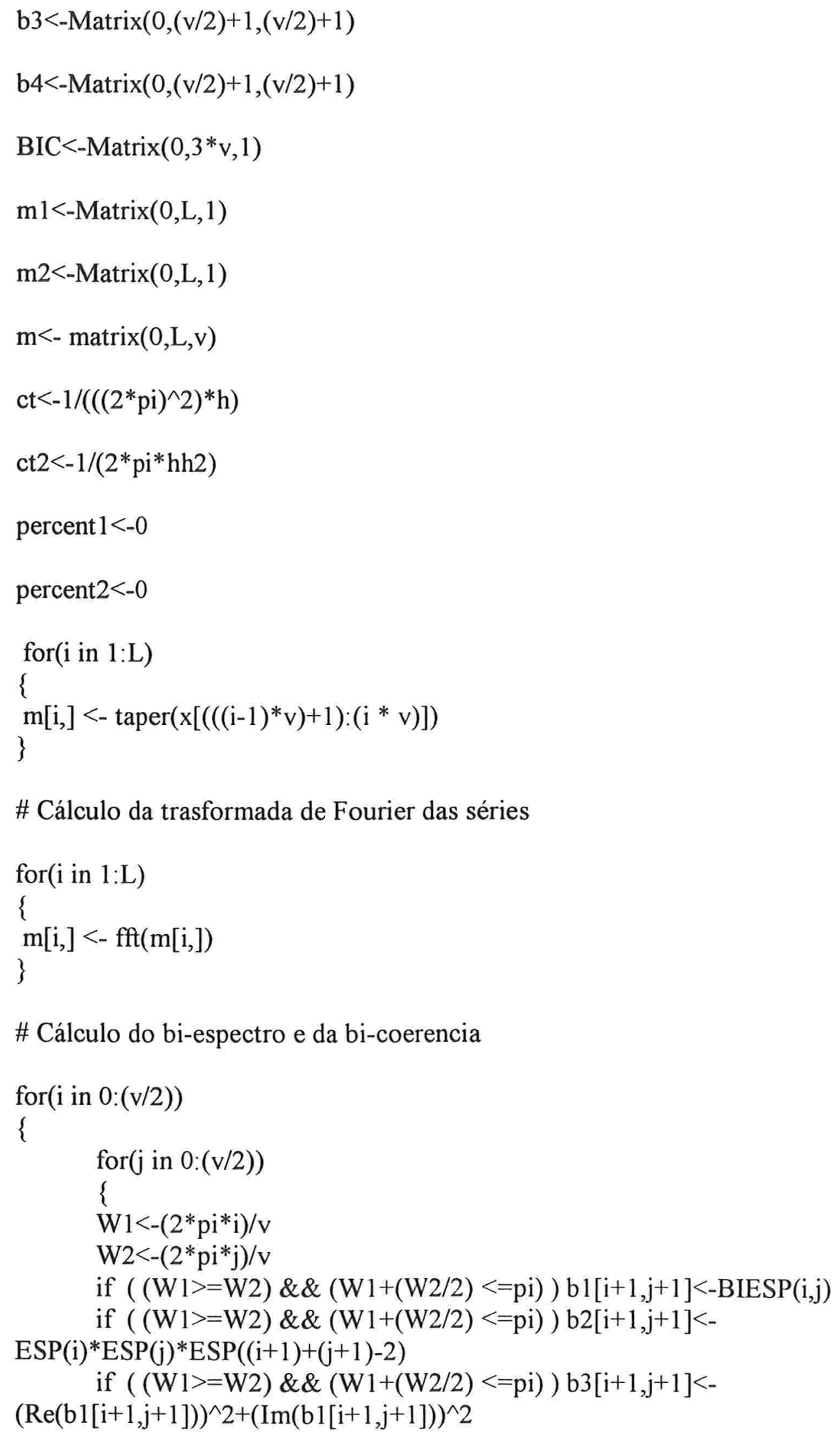




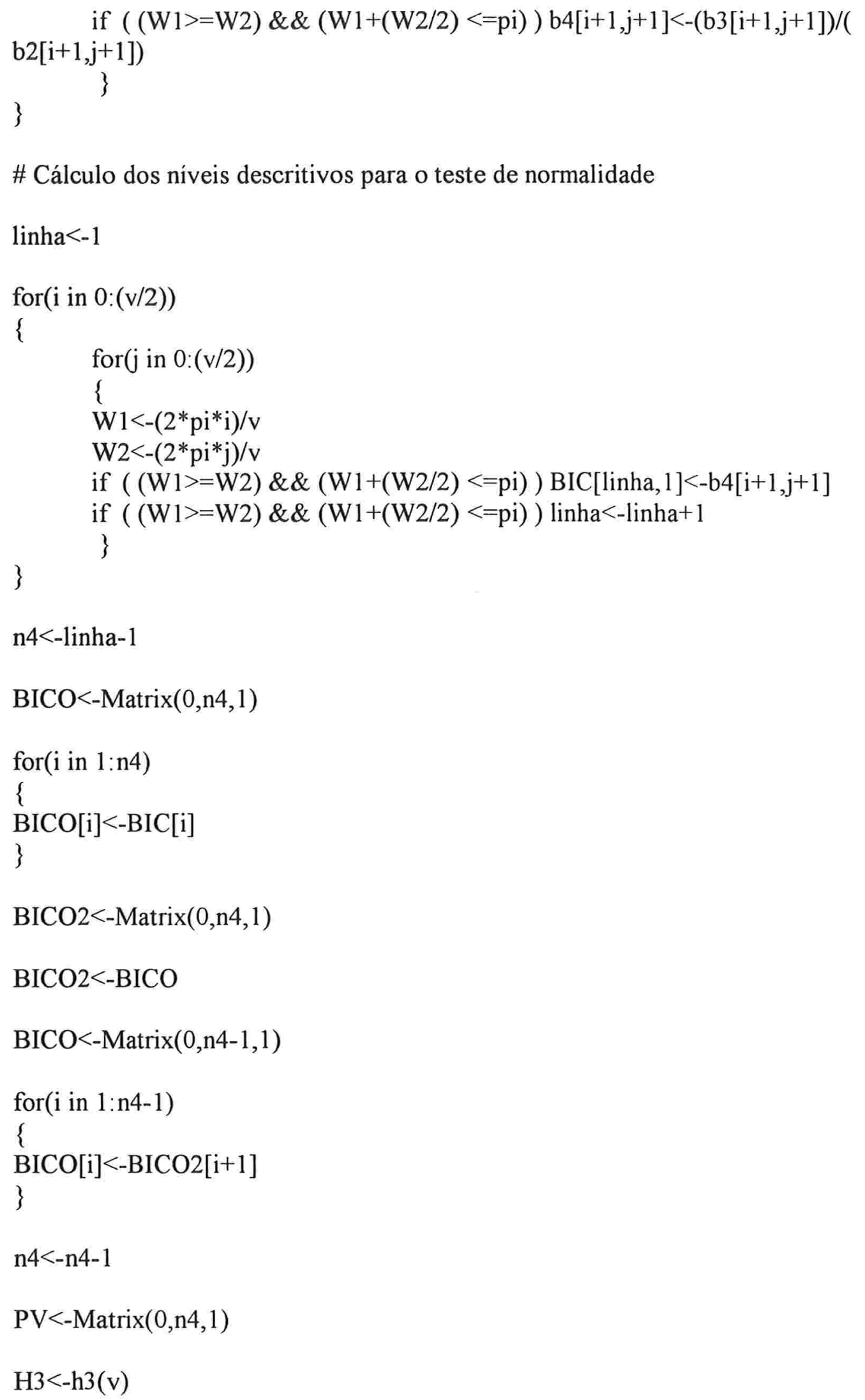




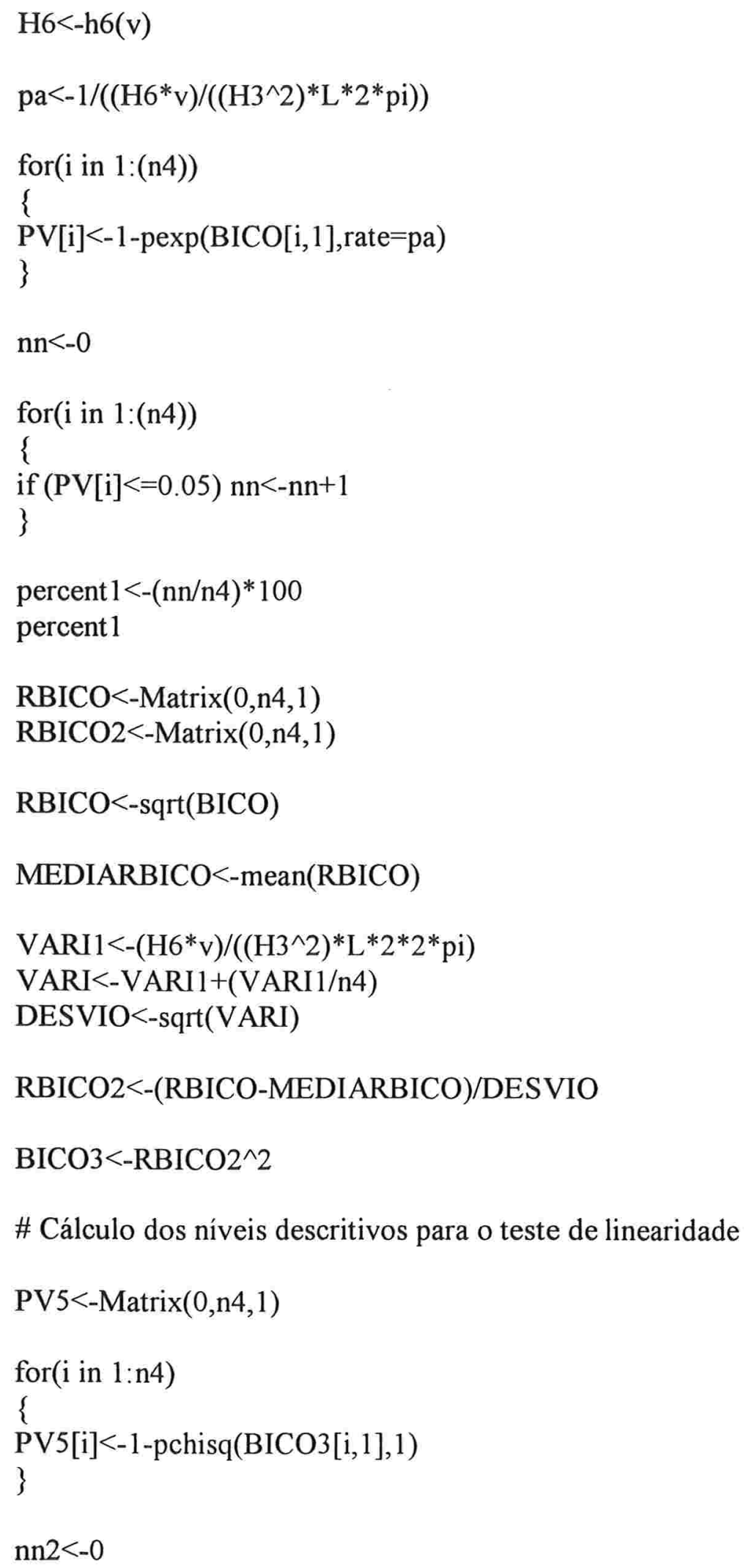


for(i in $1: n 4)$

\{ if $(\mathrm{PV} 5[\mathrm{i}]<=0.05) \mathrm{nn} 2<-\mathrm{nn} 2+1$

\}

percent $2<-(\mathrm{nn} 2 / \mathrm{n} 4) * 100$

percent2 


\section{Programa referente ao teste de linearidade e normalidade de Subba Rao e Gabr (Turbo Pascal)}

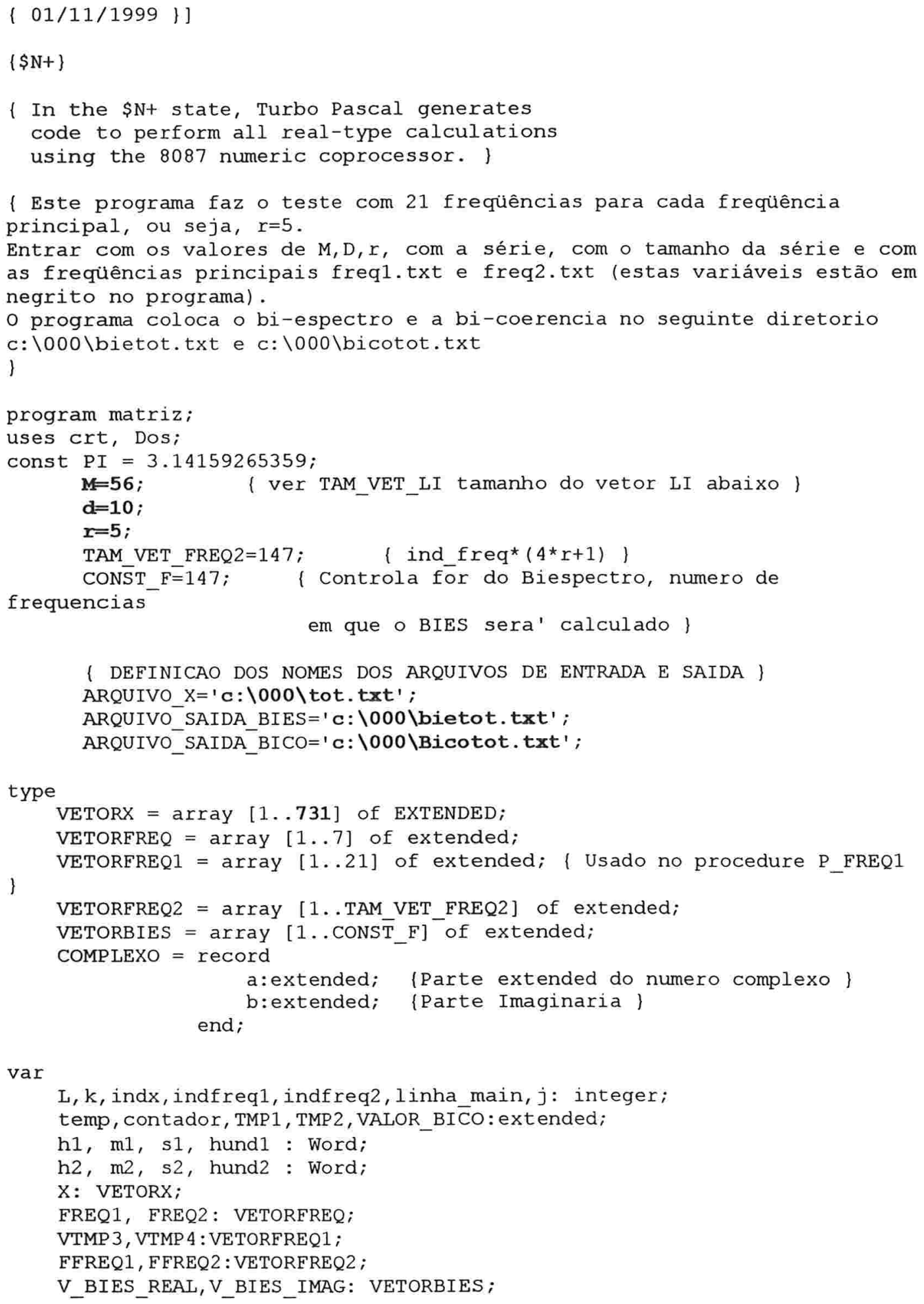




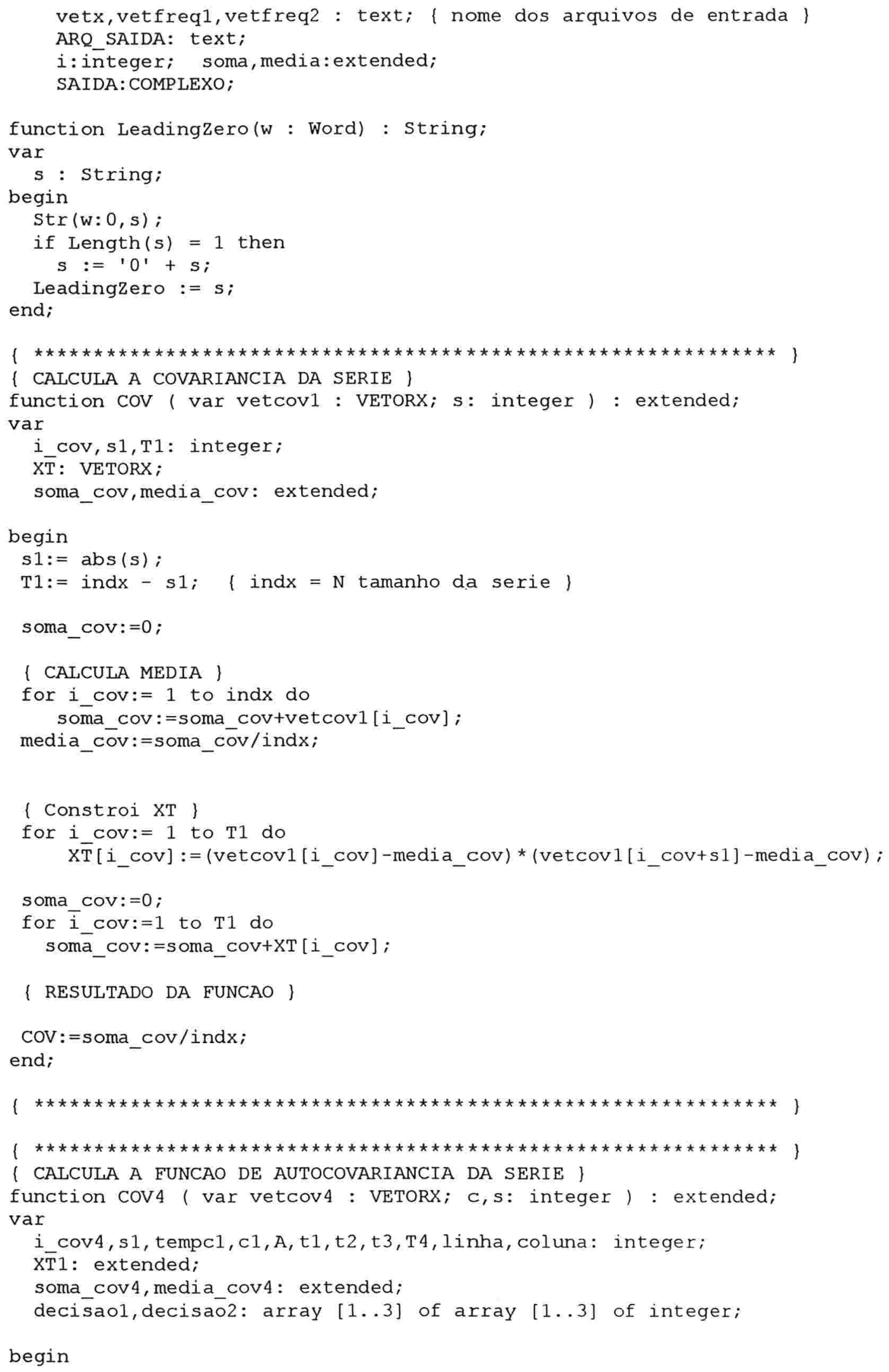




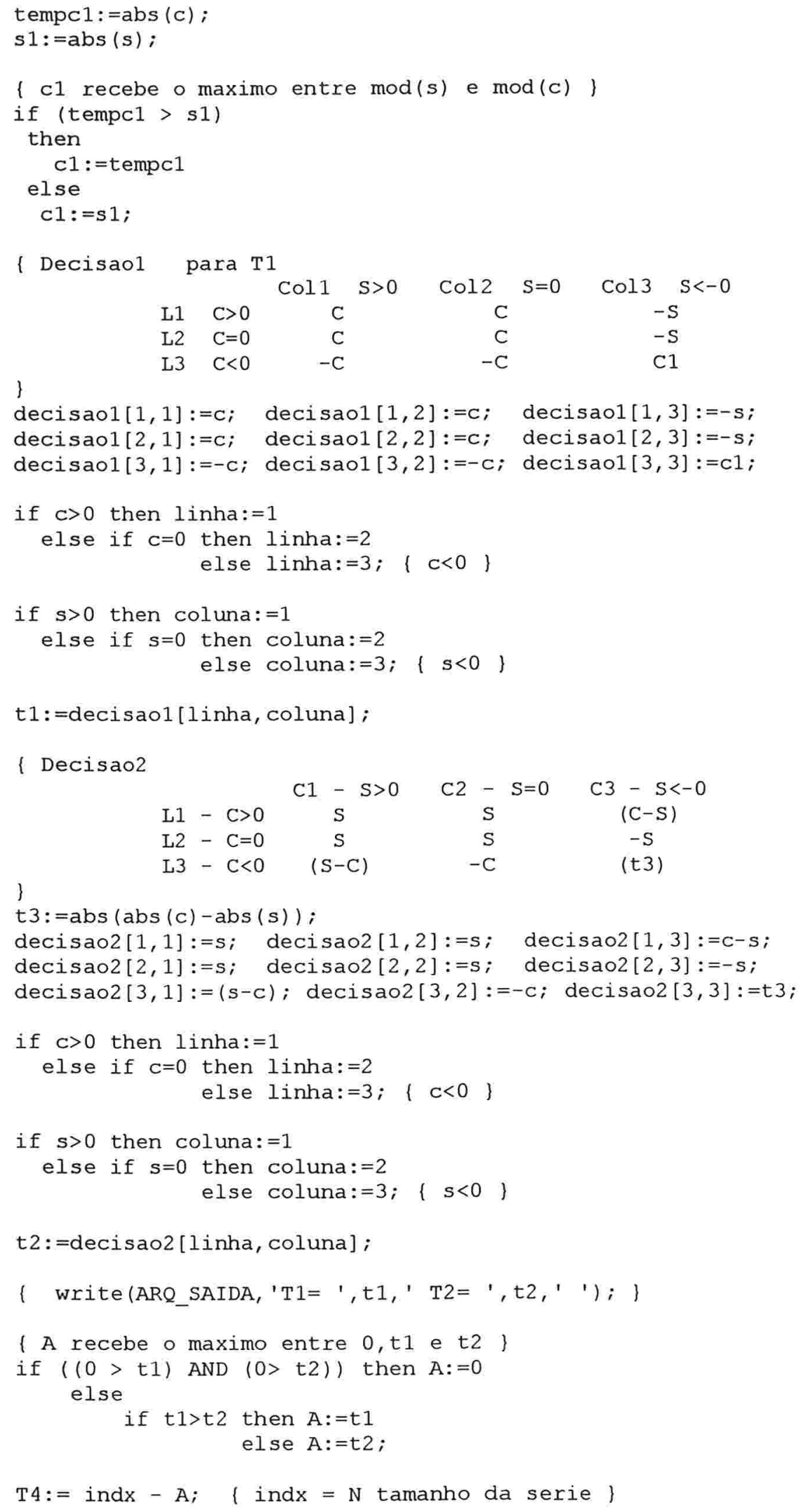




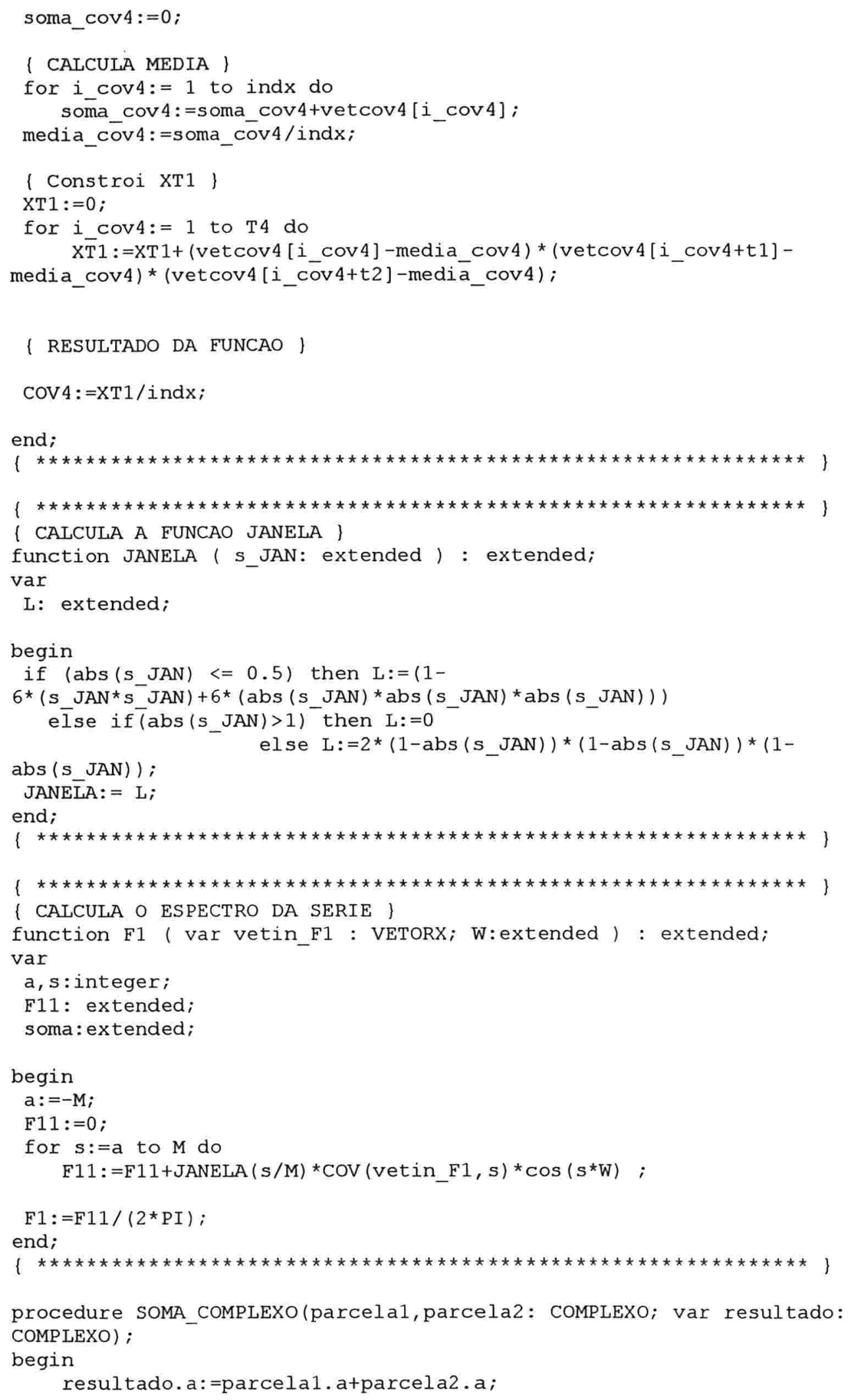



end;

resultado.b: $=$ parcela $1 . b+$ parcela $2 . b ;$

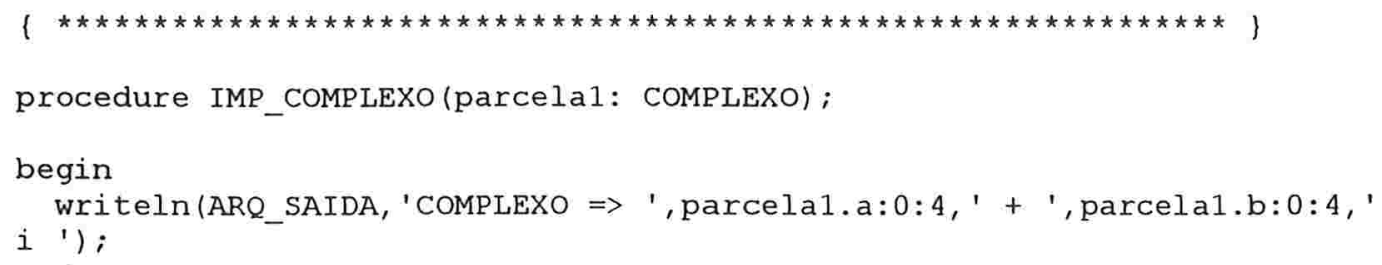


WRITE (ARQ SAIDA, 'JANELA (jj/M) $\rightarrow$ ', JANELA $(j j / M): 0: 5)$;

WRITELN (AR̄Q_SAIDA, 'JANELA((ii-jj)/M) $\rightarrow$ ', JANELA((ii-

jj)/M):0:5) ;

WRITELN (ARO SAIDA, ' COV4 $\rightarrow$ ', COV4 (vetcov, $i i, j j): 0: 5$ );

IMP_COMPLEXO'(TMP_COMPLEXO); \}

( WRITELN (ARQ SAIDA, ' (BIES) ; Parte extended TMP $\rightarrow$

' TMP_COMPLEXO.a:0:5);

\{ FIM DO DEBUG \}

parte_real:=JANELA $(i i / M)$ *JANELA $(j j / M)$ *JANELA $($ ( $i i-$

$j j) / M) * \operatorname{COV} 4$ (ve $\bar{t} c o v, i i, j j)$ *TMP_COMPLEXO.a;

SOMA_BIES.a:=SOMA_BIE $\bar{E} . \mathrm{a}+$ parte_real;

parte imaginaria:=JANELA $(i i / M)$ *JANELA $(j j / M)$ * JANELA ( $(i i-$

$j j) / M$ ) *COV4 (vet́tcov, ii,jj) *TMP_COMPLEXO.b;

SOMA_BIES.b:=SOMA_BIES.b+parte_imaginaria;

( ANTIGO LI [linha_bies]. a: =JANELA ( $i \mathrm{i} / \mathrm{M})$ *JANELA (jj/M) *JANELA ( $(\mathrm{i} i-$ jj) /M) * COV4 (vetcov, ii,jj) ${ }^{*}$ TMP COMPLEXO.a;

LI [ linha_bies] .b: $=$ JAN̄ELA $(i \mathrm{i} / \mathrm{M})$ *JANELA $(j \mathrm{j} / \mathrm{M})$ * JANELA $($ ( $i \mathrm{i}-$

$j j) / M) * \operatorname{COV} 4(v e t c o \bar{v}, i i, j j){ }^{*}$ TMP_COMPLEXO.b;

SOMA. $a:=$ SOMA. a+LI [lin̄ha_bies]. $a ;$

SOMA.b:=SOMA.b+LI [linha_bies] .b; \}

end;

resultado. $a:=$ SOMA BIES. $a /((2 * \mathrm{PI}) *(2 * \mathrm{PI}))$;

end;

resultado.b: $=$ SOMA_BIES.b/ $((2 * \mathrm{PI}) *(2 * \mathrm{PI}))$;

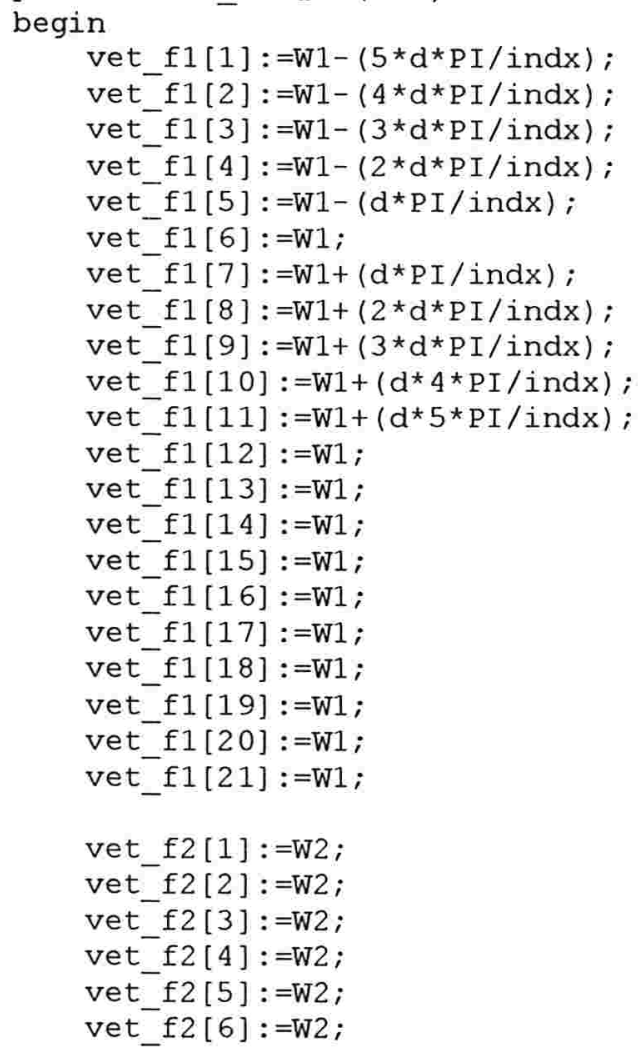




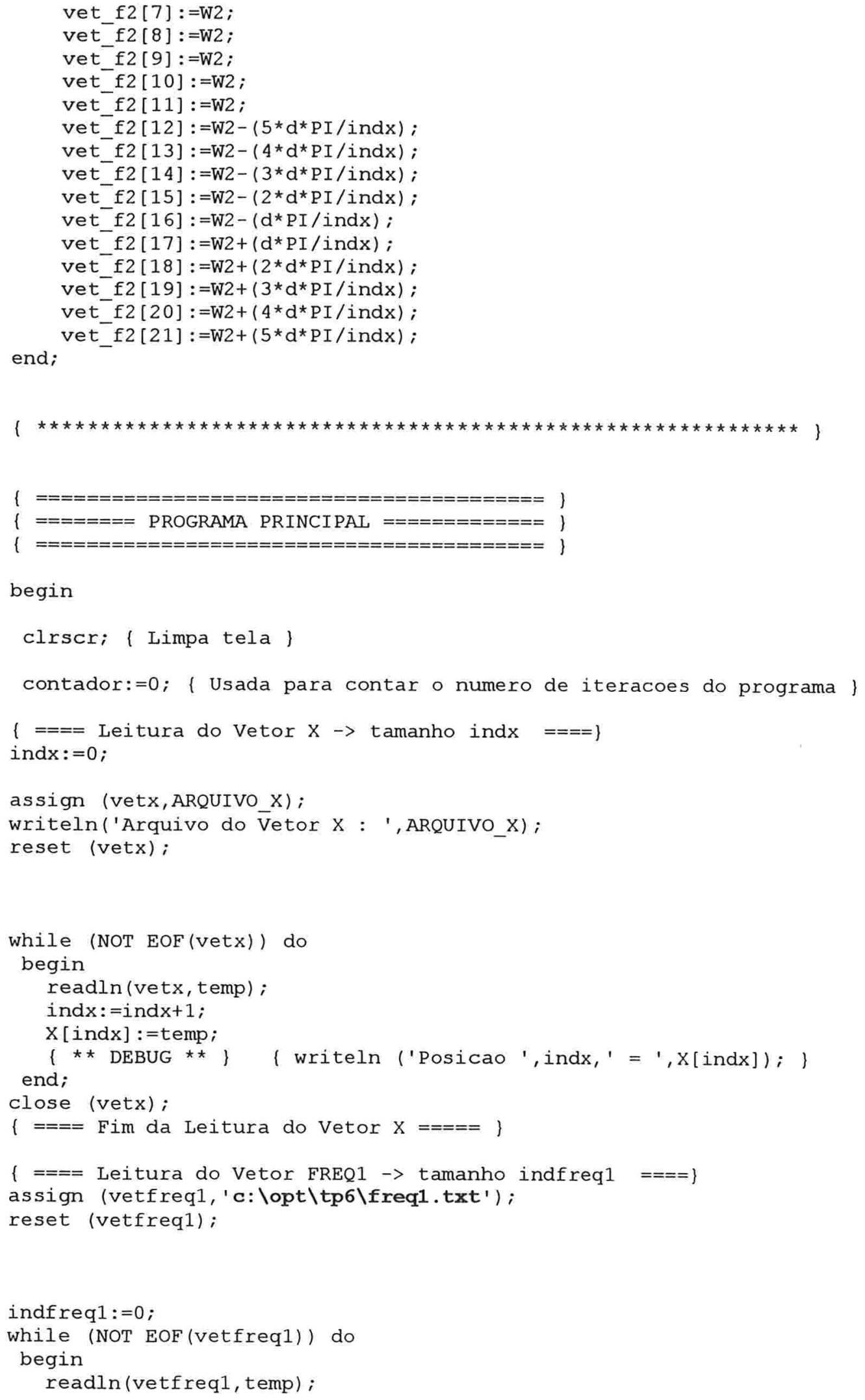




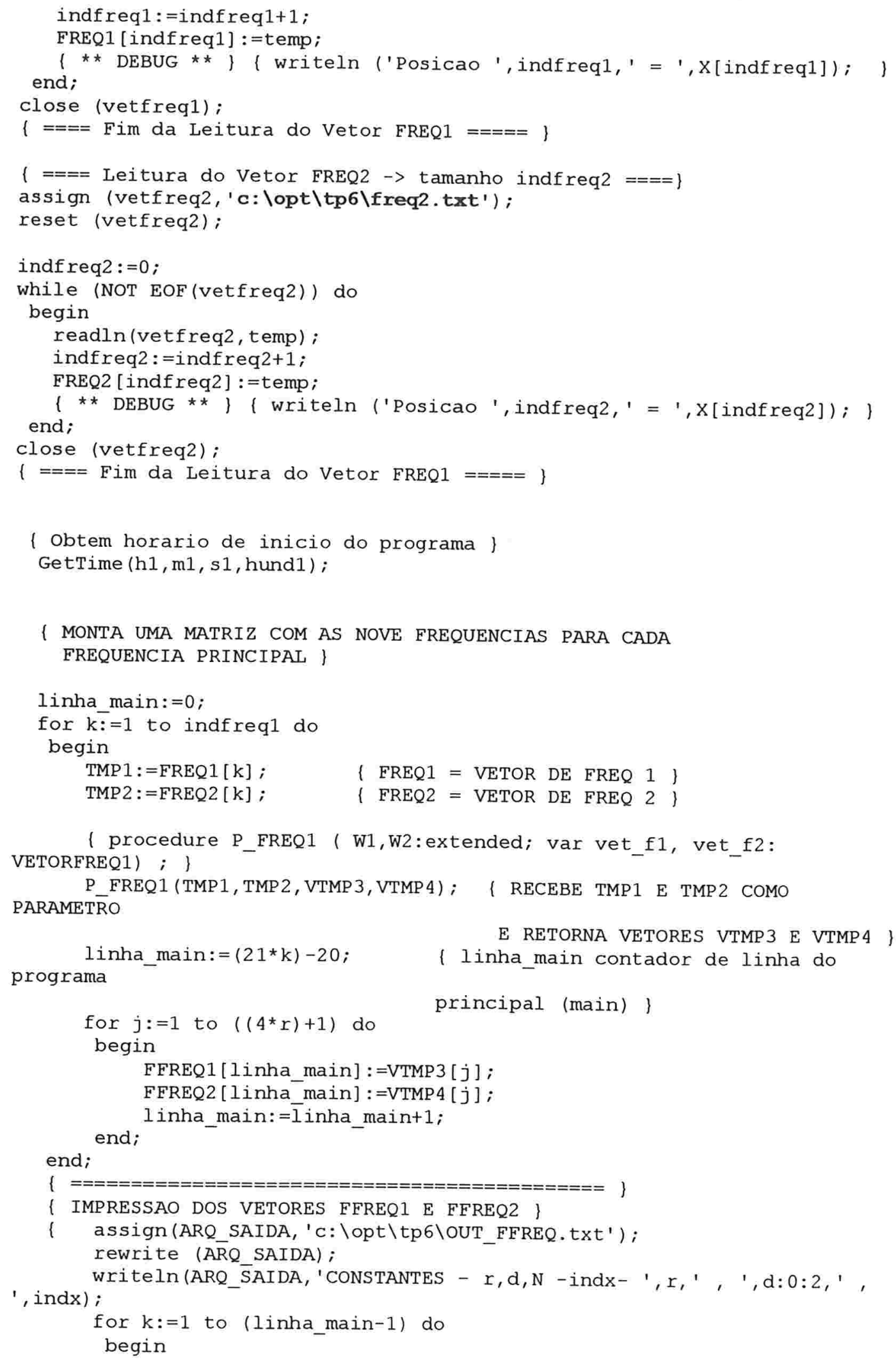




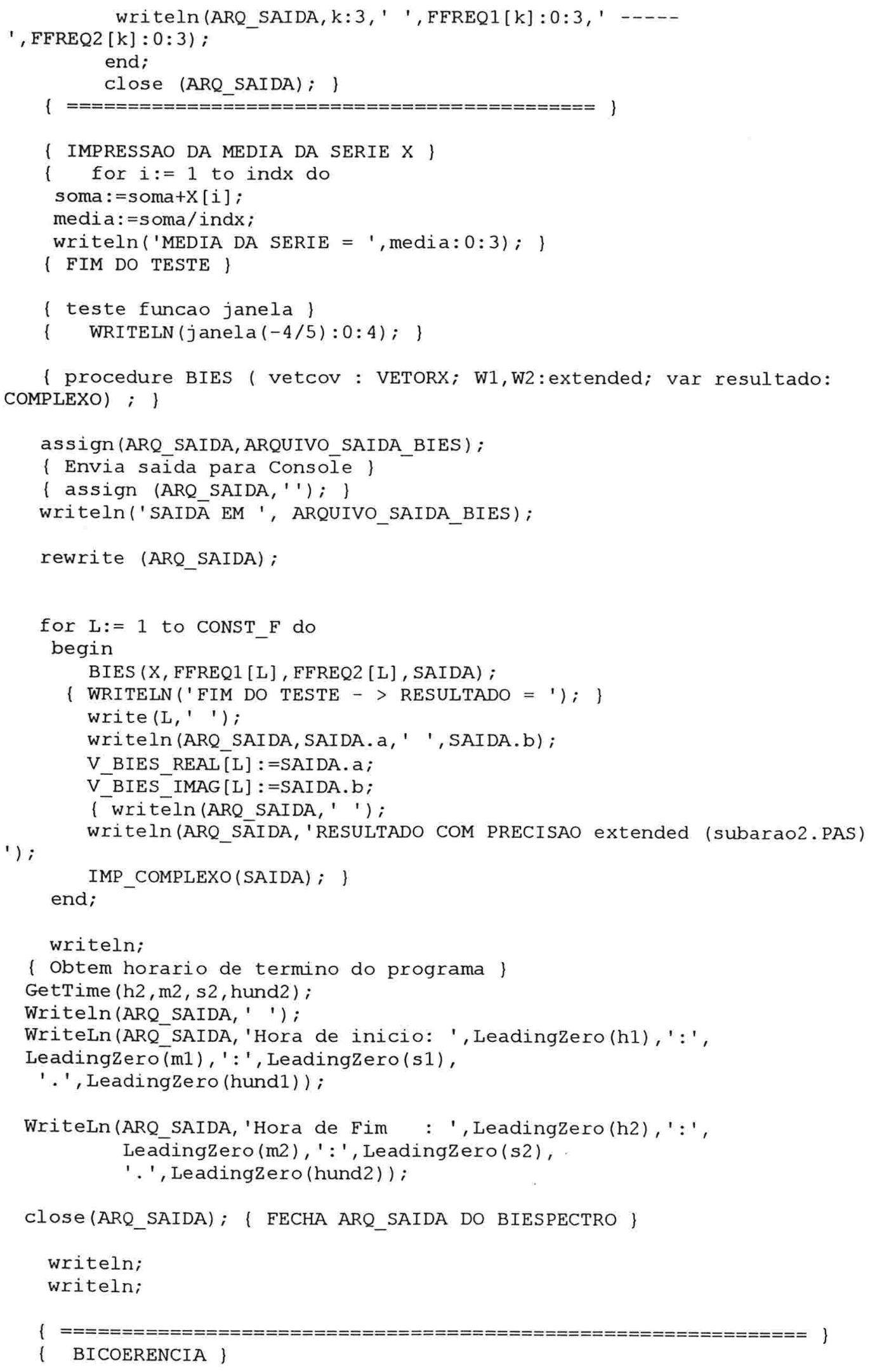




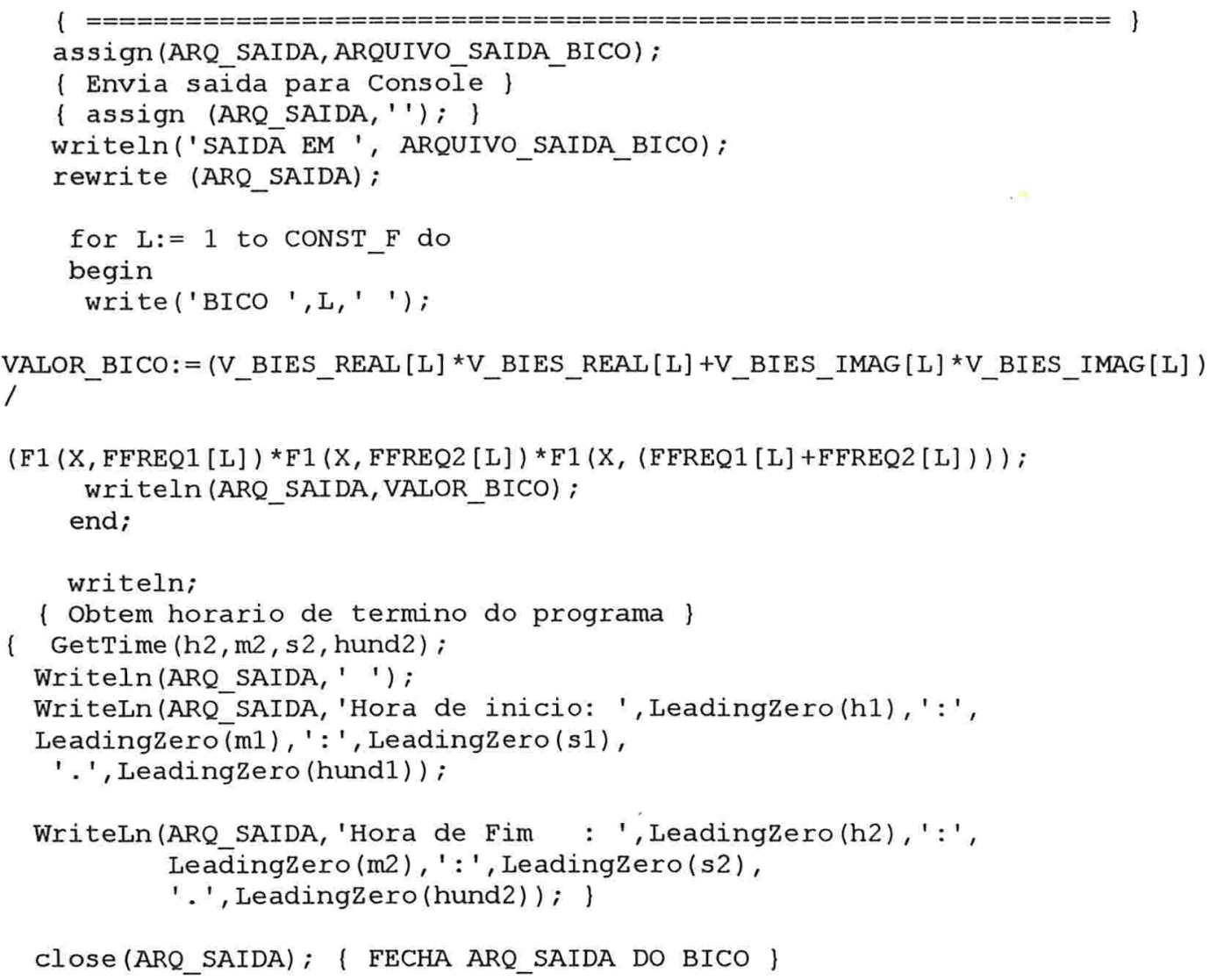

end. 